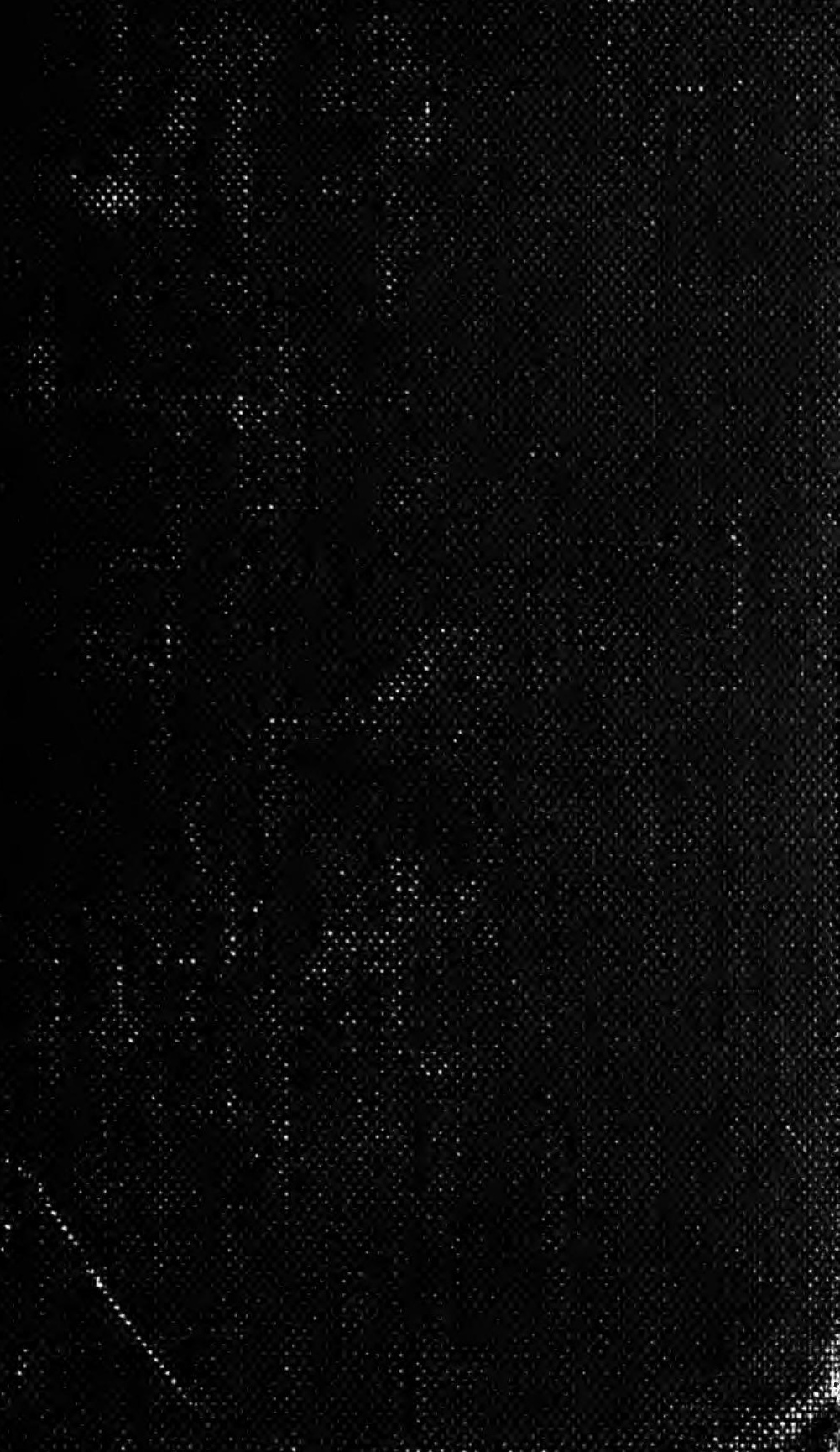



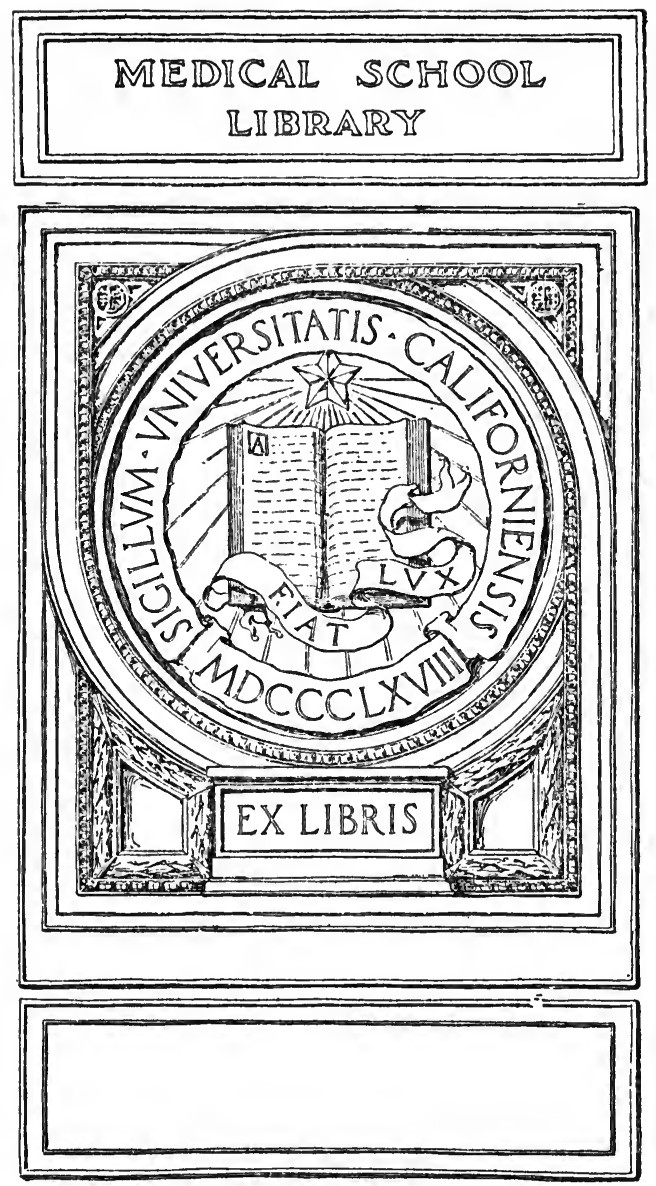

Digitized by the Internet Archive in 2007 with funding from Microsoft Corporation 


THE ACTION OF MUSCLES 



\title{
THE ACTION OF MUSCLES
}

\section{INCLUDING MUSCLE REST AND MUSCLE RE-EDUCATION}

BY

\section{WILLIAM COLIN MACKENZIE}

\author{
M.D., F.R.C.S., F.R.S. (EDIN.)
}

Member of the Council of the Anatomical Society of Great Britain and Ireland and formerly of the Staff of the Military Orthopædic Hospital, Shepherd's Bush, London ; formerly Lecturer on Applied Anatomy to the University of Melbourne, and Examiner in Senior Anatomy to the Universities of Melbourne and Adelaide

EDITED BY

CHARLES MACKAY, M.D. MAJOR R.A.M.C.

WITH 99 ILLUSTRATIONS

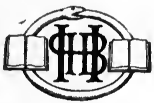

PAUL B. HOEBER

$67 \& 69$ EAST 59TH STREET NEW YORK 
Printed in England

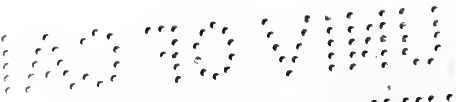




\section{INTRODUCTION}

In Army medical circles the view is held that of the wounded men who have returned from the French battlefields during the Great War, some 65 per cent. are suffering from disabilities of an orthopædic nature; in other words, whether the injury be one of muscle, bone, joint, nerve, or central nervous system, it is of such a character that the question of muscular function becomes of prime importance for purposes of treatment. When we consider that muscular tissue alone constitutes the largest part of móst animals, it is surely time that more attention was paid to the teaching of myology than has been allowed heretofore. Function of muscle-the all-important factor-cannot be satisfactorily taught in the dissecting room. It can only be taught on the living, and is largely a question of comparison-a comparison between the normal and the paralytic. Only on this plan can the question of origin and insertion of a muscle have more than an acadernic, or rather examination interest for the student.

Just as important as a knowledge of the action of a muscle is a knowledge of the action of its opponent, and the opponent should be specifically mentioned. The statement that fifteen external rotators of the hip, including such powerful engines as the psoas and great gluteus, are balanced by one muscle and part of another, can no longer be tolerated. The fact that the student when learning the action of a muscle learns that of the opponent will have an important influence on his treatment, for example, of muscular weakness, or paralysis, or of joint injuries. Thus in a case of weakness of the deltoid he will associate with that muscle the opposing adductor, namely, the pectoralis 
major. He will recognize that there can be no recovery if the opposing pectoral be allowed to contract, and will immediately guard against this occurrence.

As the muscles of the human body are adaptive or survival results of struggles which have gone on during the past ages, it follows that some appreciation of their comparative anatomy is essential for the clinician. It is inconceivable that any one should attempt to handle a paralysed deltoid or quadriceps without some knowledge of its ancestral history, or of the entities of which its function is composed.

Muscle fibre is delicate, sensitive, and responsive. It can be coaxed but not driven. It should be conserved and not indiscriminately torn or cut through at surgical operations as though of no account. In any " orthopædic" condition the muscle should be looked on as a friend, ready to do our bidding if placed under proper control. It should not be asked to do 80 units of work when it is capable of performing only 40 units. When we read in the works of H. O. Thomas such an expression as " which the muscles appear to know," we instinctively feel that when Thomas treated diseases of the hip the muscular sentinels of the joint were all personalities to him. In this way also were muscles regarded by John Hunter, Charles Bell, Haller, and Cowper, who all devoted a large part of their scientific life to the study of motion. It seems extraordinary that learned discourses should be devoted to a nerve case, and that the muscles, through loss of whose function the disease has perhaps been recognized, are so often conșidered as unworthy of even physiological rest.

The term "paralysis of muscle" is at present much too loosely applied. It would be better to state that such a condition is extremely rare. A loss of part of the function of a muscle is, on the other hand, common. There is only one true test of muscle function, the volitional test scientifically applied. Any other test, whether from the clinical or experimental point of view, is unworthy of consideration. The volitional test takes into consideration 
not only the affected muscle, but its reciprocal elongator. It is the only test by which the muscle can be considered from the point of view both of comparative anatomy and of mechanics, as is shown in the case of the deltoid and the brachialis.

Usually, the operator when he has surgically finished with a case in which muscle function is involved, passes it on for massage or electrical treatment, both of which forms of treatment are purely passive. What significance the expression massage and electricity has in the surgeon's mind the writer has often longed to know. In poliomyelitis no massage or electricity should be applied till recovery has taken place. These forms of treatment cannot initiate muscle function, but they can strengthen the action of recovered muscles. The principle cannot be repeated too often that an ounce of scientifically directed volitional effort is worth pounds of passive treatment. With this in mind it is wonderful what can be accomplished in a warm room with the aid of a table, a few pillows, and a sheet of powdered cardboard. Such indeed might be termed the Department of Myology. To this simple department the more complex ones of massage, electricity, and of those movements which owe their name sometimes to the country of origin and sometimes to their inventor, should be subsidiary. The myologist, looking at the case solely from the point of view of function, should determine whether in traumatic cases, at what stage, and of what nature, massage or electrical treatment or both would be beneficial. These should not be ordered indiscriminately and as a matter of routine. To many this may seem premature, but nevertheless it is only a question of time when a Department of Myology will be a principal feature in every Orthopædic Institution.

The writer wishes to thank the President and Council of the Royal College of Surgeons of England and also Professor Arthur Keith for the privilege of having been able to conduct researches at the Hunterian Museum during the past three years; Sir Robert Jones, Inspector-General of Military Orthopædics, for facilities accorded him in 
carrying out investigations on muscular action at the Military Orthopædic Hospital, Shepherd's Bush, and for his permission to utilize photographic and other material; the Staff of the Hospital for their kindness and co-operation, especially in allowing him to see selected cases; and Mr. Bird, artist to the Hospital, for the trouble taken with the photographs; Mr. Victor Cobb, of Melbourne, and Mr. Finnerty for their excellent drawings; and his secretary, Miss Olive Hine, for help in the preparation of the manuscript.

In conclusion, the author desires especially to thank his cousin, Major Charles MacKay, R.A.M.C., for his generous assistance extending over five years in the preparation of numerous specimens and dissections; also for his constant help and advice during the past twelve months.

While the author has been unavoidably absent in Australia, Major MacKay has kindly undertaken the editing and correcting of the proof-sheets.

\section{PUBLISHERS' NOTE}

THE gratifying reception accorded to Dr. Mackenzie's work having made a reprint necessary, the opportunity has been taken of correcting some printer's errors, while the Editor has also made some slight alterations and additions. A new drawing has been supplied for Fig. 85, and the adoption of a smoother surface paper will be found to have improved some of the illustrations in the text, which were not quite satisfactory in the first impression. 


\section{CONTENTS}

INTRODUCTION

PAGE

CHAPTER I

\section{PRINCIPLES}

I. Nature of Muscle . . . . . . . . I

II. Muscle Tendon $\quad$. $\quad$.

iil. Relation of Bone to Muscle . . . . 4

IV. ORigin and Insertion of Muscle . . . 5

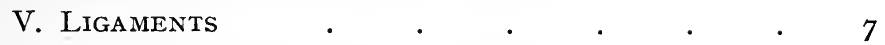

VI. Pulleys, Sesamoids, and Levers . . . 8

VII. Form and Arrangement of Muscle . . I 2

ViII. The Evolution of Muscular Action: Muscle

Re-education . . . . . . . . I4

iX. Muscle Rest: Zero Position . . . i 8

X. Chronic Muscle Shortening: Contracted

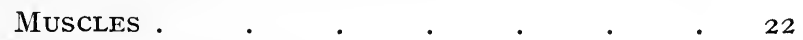

Xi. Testing for Muscle Action . . . 28

XiI. Correlations. Muscle Development and the Thymus Gland $\quad . \quad$. $\quad$. $\quad$. 30

XiII. Contraction, Relaxation, and Elongation of

Muscle : Elasticity . . . . 36

XIV. Reverse Action . . . . . . $44^{\mathrm{I}}$

XV. Muscles passing over Several Joints . . 43

XVI. Specialization of Muscle Function . 44

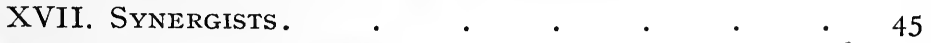

XVIII. Mechanism of Muscular Contraction • • 46

CHAPTER II

THE SHOULDER REGION . 
CHAPTER III

THE BICEPS BRACHII AND BRACHIALIS: MUSCULOCUTANEOUS PARALYSIS . . . . . . $7 \mathrm{I}$

CHAPTER IV

MEDIAN NERVE PARALYSIS

CHAPTER V

ULNAR PARALYSIS .

CHAPTER VI

MUSCULO-SPIRAL PARALYSIS • . . . • III

CHAPTER VII

THE MUSCLES OF THE THIGH .

CHAPTER VIII

MUSCLES ACTING ON THE LEG

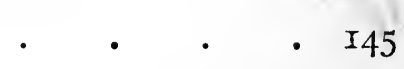

CHAPTER IX

MUSClES ACTING ON THE FOOT (GREAT SCIATIC

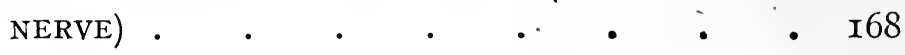

CHAPTER $\mathrm{X}$

THE MUSClES OF THE TOES (GREAT SCIATIC NERVE) • I 82

CHAPTER XI

MUSCLES OF THE SPINE.

- 200 
CHAPTER XII

MUSCLES WHICH MOVE THE NECK • • • . 206

\section{CHAPTER XIII}

MUSCLES WHICH MOVE THE BACK AND LOINS

(THORACIC AND LUMBAR REGIONS) • . . . 2 I3

CHAPTER XIV

ANATOMICAL CONSIDERATIONS IN JOINT FIXATION $\quad 222$

CHAPTER XV

THE MUSCLES OF RESPIRATION . . . . 228

$\operatorname{INDEX} \quad \cdot \quad \cdot \quad \cdot \quad \cdot \quad \cdot \quad \cdot \quad \cdot \quad \cdot \quad \cdot 25 \mathrm{I}$ 



\section{LIST OF ILLUSTRATIONS}

FIG.

PAGE

I. TENDON OF ORIGIN OF MUSCLE (MACERATED SPECIMEN) 2

2. EXAMPLE OF LEVER OF FIRST ORDER . . . IO

3. EXAMPLE OF LEVER OF SECOND ORDER . . . II

4. EXAMPLE OF LEVER OF THIRD ORDER . . . I2

5. THE LEG AND FOOT OF KANGAROO • • • • . 32

6. THE LEG AND FOOT OF KOALA (Phascolarctos cinereus) 33

7. DIAGRAMMATIC REPRESENTATION OF MUSCULAR ACTION,

AND RECIPROCAL ELONGATION • • • • 37

8. QUADRICEPS EXTENSOR PARALYSIS (INFANTILE

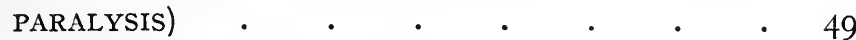

9. THE DELTOID REGION OF KOALA . . . . 50

IO. MUSCLES OF THE FORE LIMB OF THE WOMBAT (Phasco-

lomys Mitchelli) . $\quad$. $\quad$. $\quad$ facing 50

II. THE INSERTIONS OF MUSCLES MOVING THE HUMERUS • 53

I2. BI-LATERAL SERRATUS PARALYSIS OF SIX MONTHS'

STANDING .

I3. COMPLETE ELEVATION OF UPPER LIMBS WHEN SITTING

ERECT AFTER TEN WEEKS' REST AND RE-EDUCATION facing $\quad 58$

I4. POSITION FOR ANATOMICAL REST. FRONT VIEW ", 58

I5. POSITION FOR ANATOMICAL REST. BACK VIEW ", 58

I6. THE INABILITY WHEN ERECT TO ELEVATE THE UPPER

LIMBS BEYOND THE RIGHT ANGLE • facing 58

I7. METHOD OF RE-EDUCATION . . . . $\quad$, 58

I8. THE UPPER LIMB ABDUCTION SPLINT . . . 59

I9. METHOD OF EDUCATION FOR SHOULDER REGION . 6I

20. METHOD OF EDUCATION FOR SHOULDER REGION • 6I

2I. METHOD OF RE-EDUCATING THE DELTOID . - 63

22. ILLUSTRATING METHOD OF EDUCATING THE DELTOID • 64

23. STIFF SHOULDER. THE PATIENT WHEN ERECT IS

UNABLE TO ABDUCT THE ARM 45 DEGREES FROM THE SIDE . . . . . . . . . . 
FIG.

24. WHEN LYING FLAT ON A FIRM TABLE THE SAME PATIENT ABDUCTS THE ARM READILY TO A RIGHT

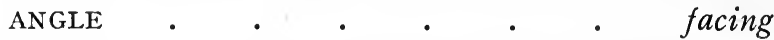

25. WHEN LYING FLAT THE SAME PATIENT CAN PARTLY

ELEVATE THE LEFT UPPER LIMB ABOVE THE HEAD,

i.e. CAN ENTER THE SECOND OR SCAPULA ROTATION ARC .

facing

26. ILLUSTRATING “ REVERSE ACTION" BY THE BRACHIA-

LIS MUSCLE

27. DISSECTION TO SHOW FRONT OF FOREARM AND HAND. KANGAROO

28. BICEPS BRACHII AND BRACHIALIS. BICEPS IN PRONA-

TION AND SUPINATION OF FOREARM . • . 76

29. NATURE'S EXPERIMENTS WITH THE BICEPS BRACHII .

30. POSITION OF REST IN MUSCULO-CUTANEOUS PARALYSIS

3I. METHOD OF REST IN MUSCULO-CUTANEOUS PARALYSIS facing 80 facing 80

32. COMMENCING RE-EDUCATION OF THE BRACHIALIS. NOTE THE SLIGHT PRONATION OF THE HAND 8I 82

33. BICEPS AND BRACHIALIS. 82

34. BICEPS AND BRACHIALIS 4 .
35. BICEPS AND BRACHIALIS. WORK FOR THE BRACHIALIS

IS INCREASED . . . . . . . facing 82

36. COMMENCING RE-EDUCATION FOR THE BICEPS BRACHII 82

37. RECOVERY OF BICEPS SUPINATION . . . $\quad 83$

38. RECOVERY OF BICEPS SUPINATION • . . . 83

39. THE MOTOR FORCES WHICH MOVE THE THUMB. . 86

40. RECIPROCAL LEVERAGE BY THE PROFUNDUS AND SUBLIMIS TO IMPROVE FLEXION POWER . • . 88

4I. POSITION OF REST IN MEDIAN NERVE PARALYSIS - 9 I

42. METHOD OF EFFECTING REST IN MEDIAN NERVE PARALYSIS . . . . . . . . facing

43. COMMENCING RE-EDUCATION OF PRONATION. MEDIAN NERVE PARALYSIS . . . . . . facing

44. RE-EDUCATION IN MEDIAN NERVE PARALYSIS • 94

45. RE-EDUCATION IN MEDIAN NERVE PARALYSIS • 94

46. METHOD OF RESTING (a) AND OF RE-EDUCATING THE

THUMB (b) IN MEDIAN NERVE PARALYSIS facing

47. RE-EDUCATING FINGER FLEXION 
48. HOW FLEXOR LEVERAGE OF THE FINGERS IS IMPROVED

BY EXTENDING (DORSI-FLEXING) THE WRIST .

49. POSITION OF REST FOR THE UPPER EXTREMITY IN ULNAR PARALYSIS

50. RE-EDUCATION IN ULNAR PARALYSIS

5I. PARTIAL ULNAR PARALYSIS • • • • •

I02

52. TESTING AND COMMENCING RE-EDUCATION IN WEAKNESS OF THE EXTENSORS OF THE FINGERS (INTEROSSEI AND EXTENSOR COMMUNIS)

53. INCREASING THE WORK OF THE EXTENSORS OF THE FINGERS

54. PARTIAL ULNAR PARALYSIS . . . .

55. MUSCULO-SPIRAL PARALYSIS (CHARLES BELL) . . I08 I09 II3

56. THE ARRANGEMENT OF MOTOR FORCES ON THE DORSUM OF THE FINGER .

57. POSITION FOR IMMEDIATE REST IN MUSCULO-SPIRAL PARALYSIS $\quad . \quad$. $\quad . \quad$. $\quad . \quad$ facing II7

58. RESTING THE HAND IN PARALYSIS OF THE WRIST EXTENDERS (THOMAS)

59. RE-EDUCATION IN MUSCULO-SPIRAL PARALYSIS . - I2O

60. MUSCULO-SPIRAL PARALYSIS . . . facing 6I. RE-EDUCATION OF THE TRICEPS . . . . I I22

62. RE-EDUCATION OF THE TRICEPS . . . . . I23

63. THE RIGHT LOWER LIMB SUPPORTING THE TRUNK IN

THE ERECT POSITION OWING TO CONTRACTION OF

THE GLUTEUS MAXIMUS AND THE QUADRICEPS •

64. FLEXION AND EXTENSION OF THE TRUNK ON THE THIGH

65. THE DORSAL ABDOMINAL WALL OF THE KANGAROO

66. THE ERECT POSTURE OF THE KANGAROO. . . I32

67. TESTING FOR PSOAS IRRITATION . . . . . . I33

68. DORSAL DISSECTION OF PLATYPUS TO SHOW GLUTEUS

MAXIMUS AND BICEPS CRURIS . . . .

69. DISSECTION TO SHOW BUTTOCK, OUTER THIGH, AND

LEG. KANGAROO

70. THE ADDUCtORS OF THE THIGH . . . . I38

7I. A SIMPLE ABDUCTION OR ADDUCTION FRAME . . I40

72. THE MUSCLES WHICH ROTATE THE FEMUR . . I43

73. Diagram ILLUSTRATING THE ACTION OF MUSClES ACTING ON THE LEG AND THIGH 
FIG.

74. DISSECTION OF INNER SIDE OF KNEE. WOMBAT (Phascolomys Mitchelli)

75. MUSCLES ON VENTRAL SURFACE OF THIGH. PLATYPUS I53

76. INSERTION OF THE QUADRICEPS EXTENSOR • • I56

77. COMPLETE RECOVERY OF QUADRICEPS ACTION (MAXIMUM WORK). AN ERRONEOUS METHOD OF TESTING FOR PARALYSIS .

78. COMMENCING RE-EDUCATION OF THE QUADRICEPS • I64

79. COMPLETE RECOVERY OF QUADRICEPS. IOO UNITS OF WORK, OR MAXIMUM .

80. ALMOST COMPLETE RECOVERY OF QUADRICEPS (SAME PATIENT AS FIG. 8I) •

8I. CONTRACTED KNEE OF TEN YEARS' DURATION, THE RESULT OF CONTRACTION OF FLEXORS OWING TO PARALYSED QUADRICEPS (SAME CASE AS FIG. 80) • I66 82. THE BENDERS (DORSI-FLEXORS) OF THE FOOT . . I69

83. THE EXTENDERS (PLANTAR-FLEXORS) OF THE FOOT - I72

84. EXTENSION BACKWARDS OF THE RIGID BODY AT THE

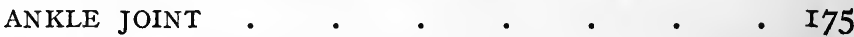

85. ACQUIRED DEFORMITIES OF THE FOOT . . . I79

86. THE MUSCLES OF THE BIG TOE (HALLUX) • • • I83

87. INVERSION OF THE FOOT IN THE EXTENDED POSITION

(TIBIALIS POSTICUS) .

88. A USEFUL FORM OF FOOT SPLINT. THE ARC SHOE - I 98

89. THE EXTENDERS (DORSI FLEXORS) OF THE HEAD. THE

ERECTOR SPIN A AND ITS DIVISIONS . facing 202

90. THE BENDERS (WITH GRAVITY) OF THE HEAD AND

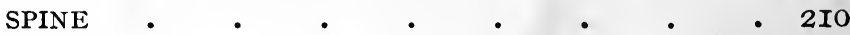

9I. FLEXION OF THE RIGID BODY AT THE KNEE JOINT • 2 I6

92. PELVIC TILTING FOLLOWING POLIOMYELITIS • • 22 I

93. THE EXTENDED ARC SPLINT • • • • • • 224

94. EFFECTIVE SPLINT FOR RESTING THE KNEE JOINT • 225

95. DISSECTION OF VENTRAL SURFACE OF THE PLATYPUS

(ABDOMEN AND THIGH) . . . . facing 230

96. INGUINAL REGION (MALE PLATYPUS) • • • 242

97. DISSECTION TO SHOW PATENT INTERNAL ABDOMINAL

RING AND CANAL CONTINUOUS WITH THE TUNICA VAGINALIS (MALE'WOMBAT)

98. DISSECTION TO SHOW INGUINAL REGION (MALE TAS-

MANIAN DEVII (Oasyurus sarcophilus) facing 244 99. INGUINAL REGION (MALE WOMBAT). • • . 245 


\section{THE ACTION OF MUSCLES}

\section{CHAPTER I}

\section{PRINCIPLES}

\section{Nature of Muscle}

THE skeletal muscles in the human body number not less than 434 and constitute the largest part of most mammals, being about $45 \%$ of the total weight. Each is a distinct organ ; each a simple independent force, endowed, since it can contract and shorten, or relax, with the power of producing motion. This power the muscle cell shares with no other in the body. Each muscle cell has an arterial, venous, lymphatic, and nervous supply-the whole constituting the motor engine. By means of the nerves extending between the central nervous system and the muscles, the force of contraction inherent in each muscle cell is so directed and regulated, as to be of greatest service in the production of movement in the body. Through the nerves also, the combination of action of separate muscles so as to improve power is effected, as for example in external rotation at the hip.

Muscular is different from elastic power, since for the production of action in an elastic body some outside force is necessary. Each muscle is primarily composed of cylindrical fibres measuring about $I_{2} \frac{1}{2}$ inches long and $\mathrm{I} / 500$ inch in diameter, and each fibre or cell has an elastic sheath or wall-the sarcolemma. These cylindrical fibres 
are held together by connective tissue which is connected to the sarcolemma, and are collected into bundles. These bundles or fasciculi are also held together by connective tissue, and collectively form the fleshy mass or muscle. The muscle is enclosed in a membranous sheath which is related to connective tissue binding the bundles. Thus forming the organ called " muscle" we have not only the special cells endowed with the power of producing motion, but also connective tissue elements endowed like the tendon not with the power of producing motion, but with that of elasticity or accommodation. This is well known, but its significance clinically does not appear to be appreciated. It has an important bearing on the question of so called muscle "tone" and " contracted" muscle.

\section{Muscle Tendon}

Usually we find that at its origin or fixed point, and at its insertion where motion takes place, the muscle belly is attached by means of fibrous tissue known as tendon. Just

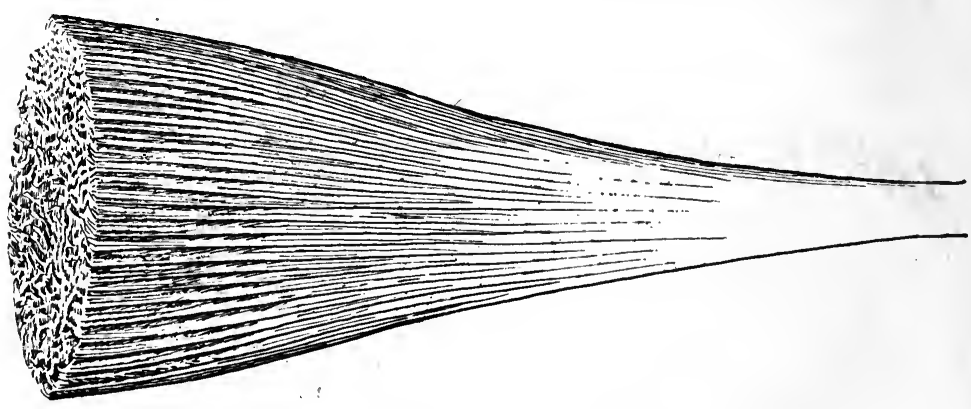

FIG. I.-Tendon of origin of muscle (macerated specimen).

as the cylindrical fibres, the fasciculi or bundles, and the muscle sheath are all united by connective tissue, so also is the tendon united to the connective tissue elements in the muscle belly, and to the sarcolemma as well. By means of maceration the tendinous origin may be traced through the muscle to the tendinous insertion. It forms with the connective tissue elements part of the elastic system of the 
muscle. It is not usual to find tendon unless there is an insertion into bone; thus it is not found in connection with the muscles of the tongue, where contractile power is brought into play throughout the whole length of the muscle. By means of tendon the force of a powerful muscle is concentrated on a small area, and in addition space is economized and limb conformity maintained, as in the case of the pectoralis major and latissimus dorsi of the upper limb. Leverage is also improved, as is seen in the long tendons of the hands and feet. Thus the muscle can act from a distance though placed near the axis of the body most suitable for nutrition and innervation. Where flexibility is not essential, as in the legs of birds, we have a bony process between the ends of muscle and the bone to be moved. Where the muscle is inserted into bone without the intervention of tendon we have power at the expense of velocity of action. Such examples are not common in the economy of the body. By the intervention of tendon between the muscle or motor force and the part to be moved, and especially owing to its elastic property, the muscle is able by this means to accommodate itself to any required movement at its fulcrum or centre of motion. A striking example of tendon or non-contractile tissue limiting the length and action of muscular or contractile tissue to the amount of motion necessary to be effected, is seen in the sterno-thyroidei of the giraffe, where alternation of these structures is present. In this way the action of these muscles in drawing down the larynx and hyoid is limited, since they would be drawn a considerable distance down the neck were tendinous material not interposed.

In general it may be stated that the greater the fleshy nature or " muscularity" of a muscle, the greater its stability and strength. To be convinced of this one has only to compare the forearm of the " selected " * marsupial - the wombat - perhaps the most muscular animal amongst the mammalia, with the forearm of a carnivorous marsupial like the Tasmanian devil, or with that of a vegetable eater

* Used in the sense of selection of the fittest. 
like the kangaroo. In the Tasmanian devil four digits only are present and tendinous substance is well defined even in the flexor belly, and in the kangaroo associated with a non-opposable thumb is a comparatively tendinous condition of the forearm muscles, which is especially seen in connection with the brachio-radialis (supinator longus). It would seem as if the necessity which evolved the " erect" posture in the kangaroo and its independence of the upper limb for support was for some unknown reason stayed. This marsupial forms an interesting comparison as regards fore limb and brain with man. Whether a muscle is largely fleshy in character, or partly muscular and partly tendinous, or mainly tendinous, has an important bearing on recovery in poliomyelitis, and especially in old cases. This condition helps to explain why fleshy muscles like those of the abdomen, psoas, muscles of the neck, and buttock recover quickly if seen early and re-educated on scientific lines, and why the muscles of the leg are amongst those most difficult to recover. It is worth bearing in mind that the tibialis anticus, which is frequently affected, is tendinous for more than half its actual length.

\section{iII. Relation of Bone to Muscle}

By means of bones and joints the results of muscular action are produced and adjusted, and the shapes and lengths of bones bear a relation to the motions performed. Bone is dominated by muscular function, for that it is called into being, with the disappearance of that necessity so also disappears the bone. Of its subservience to muscle there are numerous instances scattered throughout the animal world. Where extensive muscular attachment is required we have a broadening of the bony surface, and a narrowing or rotundity where such attachment is diminished; compactness or lightness of bone where strength or otherwise is required ; and bony ridges and projections to afford leverage. In this respect one may mention the roundness replacing the sharp tibial edge associated with an atrophied 
tibialis anticus; the bony prominence at the junction of the upper and middle third of the tibia in koala for the attachment of the semitendinosus and semimembranosus; the development of ribs with specialization of pulmonary respiration; and the relatively large development in erect man of the great trochanter for muscular attachment. It is the length, size, and "tonicity" of the muscles that maintain the normal length and size of the bones, and not the reverse, as is so often and erroneously taught. The quantity of motion in a joint is regulated by the power of contraction and relaxation of the muscles which act on it. In connection with a joint such as the knee, with an extensive range of movement, the muscles are long, in contrast to those of the spine where motion is limited. The joint must accommodate itself to the necessities of muscular function.

\section{Origin And Insertion of Muscle}

The origin of a muscle represents its fixed point or basis for action, and, speaking generally, is of the two attachments the one nearer to the spinal axis of the body. The insertion is the movable point where the effects of muscular action are produced, and is generally farther from the axis of the body than the origin. It represents the point at which the power (P) of the lever acts. Many muscles are however capable of acting in a reverse way, as for example the flexors and extensors of the hip, knee, and elbow. In this case what is usually known as the insertion is the fixed point and farther from the axis, and what is usually known as the origin becomes the insertion and nearer to the axis of the body. In general, both the origin and insertion of muscles are from bone or cartilage, although this is not always so, as for example the lingual muscles, palmaris longus, and lumbricales. The insertion of muscle is usually nearer to the fulcrum or centre of motion than the origin, even where the insertion represents $\mathrm{P}$ of a lever of the first order (stability) as in the occipito-atlantal joint, the second order (strength) 
as in the insertion of the tendo achillis at the os calcis, or the third order (velocity), which is the most common in the body, as in the flexors and extensors of the fingers and toes.

Occasionally, however, the insertion of the muscle is farther from the centre of motion than the origin, as is seen in the deltoid and coraco-brachialis. These two muscles are examples of the third order of levers, and the effects of the position of insertion are somewhat similar. to the usual arrangement in which the origin is farther from the centre of motion, since action is produced with a minimum effort. The accurate knowledge of the origin and insertion of a muscle is essential in determining its action. This applies however much more particularly to the insertion of a muscle than to its origin. It is to this that the student's attention should be directed when dissecting the muscular system. By observing the exact attachment of insertion he will be able to determine the function, but it must be remembered that, owing to the varied construction of joints for purposes of motion, the insertion of a muscle is as a rule much less easily displayed than the origin, which is usually coarser and simpler. Ocasionally, however, the origin of a muscle is more difficult to display than the insertion, as in the case of the brachialis. It is owing to the failure to recognize the importance of the part played by muscle insertion in determining action, that such ignorance is displayed when the results of the re-education of muscles, after poliomyelitis or nerve division, are demonstrated. When such muscles are treated on sound biological and mechanical lines, re-educated from a position of " zero" or minimum function, surprising results are obtained. As these do not coincide with the teachings of past centuries, they are falsely ascribed to the actions of other muscles, which from the position of their insertion could not possibly produce the results. Bearing in mind the insertion of muscle and the effects produced by volition, one should not mistake the action of a re-educated muscle for that of some other muscle, which, by its insertion, is disposed to produce the opposite effect. 


\section{Ligaments}

It would scarcely seem necessary to state that ligaments do not produce motion of a part, but exist in relation to a joint for the purposes of maintaining the necessary distance between its component parts. Owing to their elasticity they can adapt themselves to altered relations of the components of the joint, which alterations are the result of muscular contraction in response to a need or wish. They are passive agents under the influence of muscle. The muscles surrounding joints are much more important factors in strengthening them than the ligaments connecting the components of the articulation. Ligaments such as the collateral tibial (internal lateral) of the knee are liable to rupture, while muscles, owing to their power of contraction and relaxation, have greater power of accommodation to alterations of the relations of the components of the joint.

In re-educating a "paralysed" quadriceps we find that the patient can elevate the lower limb off the bed with the knee extended when lying on the opposide site, before he can complete the maximum or Ioo units of work, elevation of the limb with the knee extended when lying on the back. In the course of a public discussion of this action I heard it stated that the elevation on the side was dependent on the stiffening action of the lateral ligaments, as though the ligaments could cause movement. Again, it is often erroneously stated that talipes valgus or flat foot depends primarily on a yielding of ligaments, whereas the basic factor is muscular, an alteration in the equilibrium between the inverters and everters of the foot, dependent on a paresis or paralysis of the former. As throwing light on the origin and place in the animal economy of ligamentous bands one need only refer to the comparative anatomy of the ligamentum teres, or of the lateral ligaments of the knee. The external represents the tendon of origin of the peroneus longus when it extended to the femur, and the internal represents the insertion of the adductor magnus when it extended to the tibia. From a study of correlations 
it would appear that the long head of the biceps brachii, which prevents impaction of the head of the humerus during abduction, will ultimately be represented by an intra-articular ligament, the origin of the muscle becoming solely coracoid.

\section{Pulleys, Sesamoids, and Levers}

In the application of muscular forces throughout the body nature is ever seeking advantages to effect velocity and force of action with the minimum expenditure of energy. The shape of the muscle, the arrangement of its component fibres, the course of the tendon relative to that of the fleshy belly, its position of insertion, are all modifying factors; and it has been truly said that the human body has formed the basis of innumerable contrivances used by mechanical engineers for the conservation of force. By means of the fixed single pulley the direction of force of a muscle is altered. There is a mechanical gain, and any loss of force of action from friction is lessened by the use of a synovial sheath or bursa. The struggle of muscles for attachment undoubtedly called the pulley into being. By its means a muscle may for convenience be attached at one part of a limb while its action is being produced in a different portion. Good examples are, the cartilaginous loop for the superior oblique on its way to the eyeballthe fibrous loop at the hyoid bone for the digastric; the peroneus longus looping round the outer malleolus on its passage to the inner side of the foot; and the obturator internus which, though arising in the pelvis, is enabled to act on the upper end of the femur by means of a groove on the inner surface of the ischial tuberosity.

The more parallel the termination of a muscle is to the axis of the bone into which it is inserted the weaker its action-the maximum effort is obtained when the line of its resultant forms a right angle with the bone into which it is inserted. Thus when the elbow is extended or acutely flexed the moment of force of the brachialis is at its weakest 
- the greatest moment corresponds to the position when the forearm is at a right angle, the brachialis then acting almost perpendicularly on the ulna. Thus in testing for recovery in a " paralysed " brachialis the forearm should be passively brought to the right angle with the patient lying down. Unfortunately the test usually given to a weakened brachialis is to ask it to do Ioo units of work; the patient is asked to bring the hand to the mouth while sitting or standing, with the elbow extended and the hand at the side. Notable examples in the body by which this principle of the pulley is effected are the patella, through which the main force of the quadriceps acting through the ligamentum patellæe is increased by being raised from the centre of motion at the knee ; and the sublimis and profundus tendons of the fingers where each in turn acts as a patella to the other. I have little doubt also that this is the reason why the latissimus dorsi near its termination passes over the lower angle of the scapula.

A very interesting example of the production of an artificial pulley is seen in connection with the above action of the flexores sublimis and profundus. As is well known the position of the hand for a strong flexion grip is that of extension (dorsi-flexion) at the wrist joint; and when in the case of a diseased wrist ankylosis is the desideratum, this is the position of greatest utility. The grip becomes weaker and flexion power becomes lessened, as we approach the position of acute flexion. This important point is made use of in the re-education of the flexors in a case of median or combined median and ulnar paralysis. The position of dorsi-flexion of the wrist is independent of the muscles which move the fingers. It is caused by the contraction of the two radial extensors and the extensor carpi ulnaris, with the relaxation of the flexor carpi radialis and flexor carpi ulnaris. The result of this position is that the flexores sublimis and profundus tendons are enabled to enter the hand in such a way, that the portion in the forearm forms an angle with the manual portion, the wrist becoming a fixed pulley over which the tendons play. In a case of 
median and ulnar nerve repair after complete division in the arm, I found during the process of re-education, that although the patient flexed readily in the dorsi-flexed position; yet when the wrist was in the position of equilibrium as regards the flexors and extensors, the power of contraction of the fingers became lessened, and when the wrist was bent the patient was unable to flex the fingers even to a slight
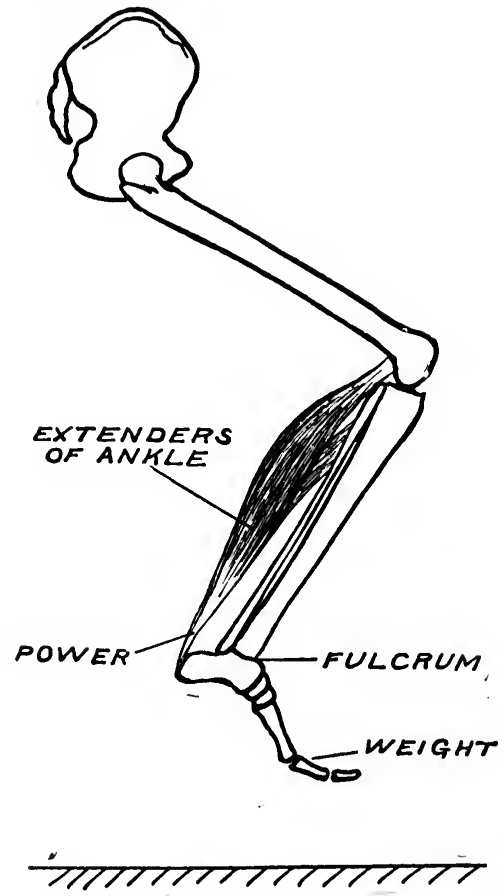

FIG. 2 .

extent. The action of the lumbricales (the flexors of the metacarpo-phalangeal joints of the fingers) which take their origins from the profundus tendons would be similarly affected.

In the human body the bones which give attachment to muscles, the muscles by which motion is produced, and the joints by which the bones are enabled to move on one another, are found to conform to a system of levers arranged in three 
orders. F represents the fulcrum or centre of motion at the joint, $\mathrm{P}$ the power of muscle contraction applied at the insertion of the muscle, $\mathrm{W}$ the resistance to be overcome. In a lever of the first order the fulcrum lies between the weight to be moved and the power. A good example of this order is found in the articulation of the head on the atlas. Thus in extension of the head the power is applied by the extensors of the head, the fulcrum is in the middle

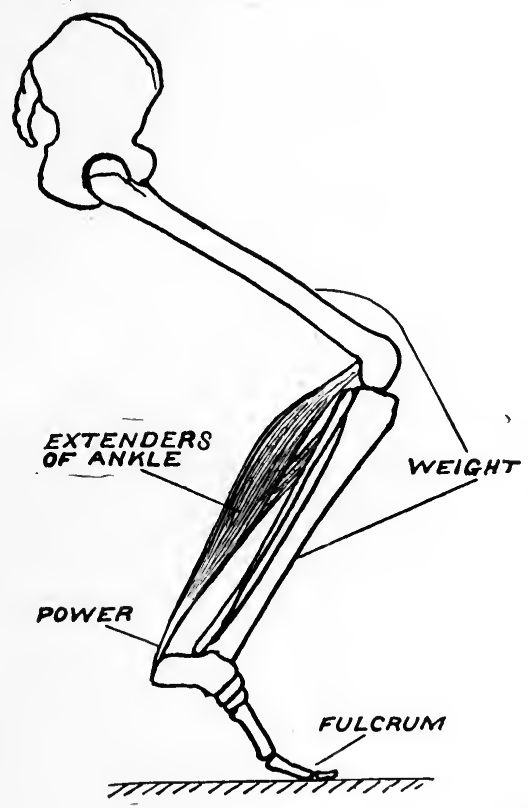

FIG. 3 .

at the occipito-atlantal joints, and the weight to be lifted is anterior. The feature of this arrangement is stability, but it must not be supposed that this stability depends on the bony arrangements and not on the muscle. In a case of poliomyelitis affecting the flexors the head is drawn backwards on the spine, and in unconsciousness the head tends to fall forwards. Another good example is seen when we lift the foot off the ground, and point the toes downwards by extending the ankle (fig. 2). Here the 
fulcrum or centre of motion is the ankle, the power the attachment of the tendo achillis at the os calcis, and the weight or resistance the front of the foot.

In a lever of the second order $\mathrm{W}$ lies between $\mathrm{P}$ and $\mathrm{F}$. This variety is not common. It is the lever of power as compared with velocity - the power arm being longer than the weight arm. The best example in the body is seen (fig. 3) when a person stands on tip-toes and supports the body on the ball of the toes, which is the fulcrum-the resistance or weight is that of the body transmitted through the tibia on the astragalus-and the power is applied to the os calcis or calcaneus by the tendo achillis.

In the third order, the common variety of lever, $\mathrm{P}$ lies between $\mathrm{W}$ and $\mathrm{F}$. It is the lever of velocity as compared

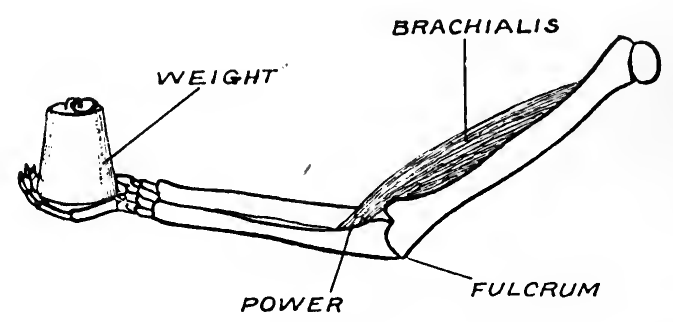

FIG. 4.

with power, for the power has a shorter arm than the weight. A good example is seen (fig. 4) in the action of the brachialis in bending the elbow with a weight in the hand. The elbow joint is the fulcrum or centre of motion. The power is applied at the insertion of the brachialis into the coronoid process of the ulna, and the weight to be lifted is the forearm and hand.

\section{Form and Arrangement of Muscle}

The muscles of the body present a great variation in size and shape. They may be broad or narrow, long or short, round or flat, thick or thin. Speaking generally, in the case of the limbs the superficial muscles, such as the hamstrings, are longer than the deep, and the deep muscles are the 
broader, as seen in the case of the pronator quadratus and popliteus. In the trunk, as is evidenced by the greater area for attachment, the muscles are broader than in the limbs. There is also a great variability throughout the muscles of the body in the arrangement of the true contractile fibres relative to the tendons to which they are attached. It is not possible to find in the body a muscle whose fibres run parallel to each other from the origin to the insertion. Perhaps the nearest approaches to this rectilineal type are the pronator quadratus and the thyro-hyoid. With this arrangement too much muscular energy is expended to produce a desired result, since the line of the resultant of forces of the muscle is the same in the tendon as in the fleshy belly. It is interesting to note that though the pectineus approaches the rectilineal type, the expenditure of energy is lessened and velocity improved, owing to the fact that the planes of origin and insertion are not on the same level but intersect.

Speaking generally the arrangement in the skeletal muscles is such that the fibres do not go straight from origin to insertion, but are directed obliquely. By this obliquity, as is shown in the action of the intercostal muscles, is produced a more rapid action with a smaller contraction, and a lessened expenditure of energy, than if the fibres were continued in a straight line to the tendinous insertion. By this arrangement also liberty of limb motion is freely obtained. The nearer the deviation of the muscle, relative to the direction of its tendon of insertion, approaches the right angle, the less is the contraction necessary to produce the desired effect. The oblique arrangement of fibres is well exemplified in muscles like the peronei where the fibres are attached to one side of a tendon like the plumes of a pen. This is the so called half-penniform arrangement; or we may have an additional course of oblique fibres, which converge obliquely on each side to a single medial tendon, by which arrangement their power is increased. This is the complete penniform or bipenniform arrangement, and the best example in the body is the rectus femoris. In the case of the deltoid 
the belly is strengthened and action improved by the interposition in the belly fibres of tendinous bands. In triangular muscles the fibres arise over a broad origin, but the insertion is a narrow one. Good examples of this are the pectoralis major-temporal-and the gluteus minimus. By this arrangement powerful muscular action can be concentrated on a narrow area.

\section{ViII. The Evolution of Muscular Action: Muscie RE-EDUCATION}

It is customary for us to speak of the action of any given muscle as though it represented a single entity, forgetting that the muscles of man are "selected" results following struggles which have extended over many ages. Thus we speak of a flexor or extensor of a joint, as though these terms conveyed everything necessary to explain function.

My attention was first drawn to this question more than ten years ago by studying the action of the quadriceps, deltoid, and brachialis muscles in cases of poliomyelitis. In this disease examples of quadriceps paralysis were met with in which the patient was unable to stand, or, when lying in bed to raise the heel with the knee extended; however, when lying on the side opposite to that which was affected, and the knee then being flexed, it was found that the leg could be extended with ease. Because a patient is unable to raise the heel off the ground with the knee straight when sitting on a chair-is unable, that is, to do roo units of work-we are not justified in calling the quadriceps " paralysed." On the same reasoning the quadriceps tested with the patient in bed and with the knee acutely flexed might be regarded as normal, since it can perform the function of the quadriceps as given in the text-books, that is to say, extend the knee.

In the case of the deltoid it is not infrequent in connection with poliomyelitis or trauma to find a patient who is unable to elevate the arm when standing or sitting erect. The deltoid is condemned as hopeless for recovery, since it has failed to respond to treatment by massage and electricity. 
Yet such a patient if lying flat on the back may be able to raise the upper limb above the head with ease, and by gradual elevation to the erect posture may, within a few months, be able to raise the limb above the head when standing erect. Or we may find a patient with a paralysed brachialis who is unable when tested sitting or standing erect to bring the hand of the affected side to the mouth. But the same patient when lying on the back with the limb supported by a pillow in the manner which I will subsequently indicate may be found able to flex the forearm readily from full elbow extension. These phenomena admit in my opinion of only one explanation, that the disease or injury is revealing functional stages in the evolution of these muscles. They show that muscle action is a complex function, one made up of several separate activities, and hence the term "paralysis" of muscles is really referable to the loss of some, not necessarily all, of their functions. The recovery like the loss follows in an ancestral or evolutionary sequence.

Frequently one hears the term "irreparable damage" applied to central nerve cells in cases of poliomyelitis. Such a term, unless qualified, is not only meaningless but misleading. "So called "irreparable damage" can only be recognized by the degree of muscle "paralysis" present, and this depends upon the nature of the test applied to the muscle. Thus "irreparable damage" might be applied to the cell condition when the quadriceps failed to contract sufficiently to enable the patient to elevate a lower limb off the bed in one piece, yet such a quadriceps tested from "zero" might show every other function to be present. " Irreparable damage " and " paralysis" are really referable not to the muscle, but only to the loss of part of its function ; the restoration of which follows a biological law. So called "irreparable damage" must be judged by the loss of all functions of a muscle-all must be tested, not some or any. Thus when we speak of recovery in a deltoid or a quadriceps it is necessary to define exactly the extent of the recovery. Do we refer to roo units of work-the work-power of the corresponding normal muscle of the other limb-or to only 
50 ? The above method of testing, which is based on a scientifically directed volition, is the only real test for muscle recovery. It is the only one which takes into account the contracting force and the relaxing opponent. Do medical men seriously believe that an externally applied electric current has the power of eliciting functional entities of a muscle or of discriminating between them ? It is this volitional test which should primarily tell us whether an operation on a motor nerve suspected of injury should be performed or not.

The assumption of the erect posture has had a dominating influence on the muscles of the human body, and the study of muscular action throughout the mammalia shows that the orthograde functions have been superimposed on muscles adapted originally for plantigrade motion; new muscles have not been called into being. Although Charles Bell taught us that the anterior cord presides over muscular action, and Marshall Hall that movement can occur reflexly as well as from volition, a study of cases of infantile paralysis in man also demonstrates the fact that the anterior cord presides not merely over " muscular action," but also recognizes the components of which a function is made up, and that these cell-controlled components of a purposive movement always bear a definite sequence one to arother.

The action of the quadriceps is vastly different in platypus and in man. The quadriceps in platypus corresponds to the anatomical test of extension of a flexed knee, but that is a distinct function from that of a quadriceps which will allow of the orthograde posture. The quadriceps of the ape has a more complex function than that of the platypus, and so similarly has man's compared with the anthropoid. A patient who can raise the heel off the ground when sitting with the knee extended will be able to extend the acutely flexed knee in bed; but the patient who can do the latter need not necessarily be able to do the former. When, therefore, we are told that the quadriceps is paralysed merely because the patient is unable to raise the heel of the extended leg off the ground, we are told something which is biologically 
untrue. This is a question apart altogether from volition, since in infantile paralysis we are dealing generally with a disease, in which cerebration is unaffected. The koala shows us a typical exhibition of deltoid function amongst the marsupials. Compared with the deltoid of the wombat that of the koala has become differentiated from the outer pectoral, trapezius, and brachio-radialis (fig. 9). Although the koala, supporting himself with one hand in the fork of a tree, can reach for his meal of eucalyptus leaves, that is a different matter from the ability to raise the arm when the orthograde posture is assumed. Again, a study of the anthropoids at any menagerie shows with what reluctance they dispense with the fore limb as a means of support.

The ape prefers the knee slightly bent, with the balance afforded by one or both fore limbs. To assume the orthograde posture is an effort, and it is a still greater one while in the upright position to raise the fore limb above the head. The stiffening of the knees by the quadriceps-which has enabled man to stand and walk - and the ability not only to dispense with the fore limb for support, but to elevate it when erect above the head, are late acquisitions in man's evolution, and are hence unstable.

It is such observations as these that have induced me to formulate the evolutionary law of muscle function. The particular functions of any muscle are the result of the evolutionary history of that muscle.

Compared with embryology, which gives us an imperfect picture of man's evolution from the point of view of structure correlated with function, comparative anatomy not only gives a connected history of the past phases, but often points the directions in which man's future physical evolution may progress. For example, it will I think be conceded that inversion and eversion of the human foot are diminishing, since the foot is not, as in koala, provided with an opposable hallux; also that the tibia is the dominant bone of the leg, and that the fibula is disappearing. The fibula in the kangaroo, from the point of view of function, is non-existent, having become approximated to the tibia ; reminding one of 
the manner in which the appendix has become fused with the ileum in the wombat. In the kangaroo a struggle exists round the ankle, as elsewhere, between individual muscles, not only for position but for existence. The kangaroo's foot can be regarded, then, as of especial value in enabling us to recognize that certain muscles are " survival results."

Even if the "paralysed" patient be treated at a stage of the disorder when some surgical interference is necessary, the comparative method of treatment should still dominate the surgeon's action, whether the method be the " strengthening of the weak" by muscle or nerve transplantation, or the " weakening of the strong," as by tendon lengthening or division. If we are utilizing a tibial or peroneal muscle for grafting, it is important to know which tibial or which peroneal is the more useful in the body economy. Similarly, if we are diminishing the power of eversion because the tibials are weak, or inversion because the peronei are damaged. The results obtained from nerve and muscle grafting in paralysis have so far been disappointing, and for the reason that muscle function-the important factor-has usually received such scant consideration. It seems ridiculous to graft a biceps femoris or other hamstring to the quadriceps and expect it to restore extension-that is, to repeat the biological history of the function of that muscle-unless we have a clear idea of the entities of which that function is composed. Again, presuming we have reinforced the circumflex nerve of a paralysed deltoid, it is surely too much to expect that nerve impulses alone will stimulate the muscle to repeat in sequence the events of its evolutionary history.

\section{Muscle Rest : Zero Position}

From the scant attention paid by clinicians to muscle rest in diseases of the nervous system it would appear that rest-and by that is meant physiological rest-as a curative agent is supposed to be unworthy of consideration as part of treatment. To many it appears to be of no importance 
that the muscle is really a part of a nervous mechanism. The motor nerve and the muscle are functionally interdependent. It is true that the source of motion resides in the muscle, but the cause of voluntary movement resides in the nervous system. Through nerves the will power is conveyed, but nerves have no power of motion. Both the nerve and muscle are best considered as parts of an original unit, the muscle retaining the power of contractibility, of producing motion, and the nerve retaining the power of irritability and of conduction. In a disease like poliomyelitis, where the chief trouble falls on the anterior cornual cells, treatment has always been directed towards maintaining the nutrition of muscles by means of electricity and massage, in the hope that the cells might ultimately recover, when the muscles presumably would be in a condition to resume function. If the muscles under such treatment did not recover no fault was found with principles of treatment, but it was supposed that the cornual cells were irreparably damaged. The atrophied deltoid with flattening of the shoulder and a dangling upper limb were unfortunate but unavoidable results.

If rest is the basic treatment of inflammation, and towards this we must suppose that nature is always striving, it is clear that the cornual cell can be best rested through the muscle. With constant irritation of the muscle by faulty position, massage and electricity, the utmost is being done to prevent recovery of the inflamed central nervous system. It is interesting to find that when we rest a deltoid and ask it gradually to resume work within functional limitations, it does not waste as was supposed. The fallacy that we must do something to the muscle "to maintain its nutrition" would appear to be dying as hard as the one that by resting a joint its liability to ankylosis is increased. If a motor nerve be injured, the injury has also affected the muscle, and how can we expect recovery of the muscle after repair of the nerve, if we fail to rest the muscle? Not only is muscle rest the most effective agent for repair of injury or inflammation of either muscle itself or of the nervous system with which it 
is connected, but the position of rest is the physiological basis for the re-education of muscle function. It cannot be too strongly insisted on that in injury of a limb, as from a bullet or shell wound, our first care, apart from antisepsis, etc., of the wound itself, should be the immobilization of muscles. Future muscle function should dominate fixation, and the sooner the limb is rested from the point of view of its musculature, the greater the ultimate chance of utility.

Effective rest of muscle should begin at the time of the receipt of injury. This will minimize the necessity for operative interference and give the limb the best chance should an operation be performed. Immediate protection of muscles by effective rest is fundamental in the treatment of injury of a limb. It is a matter for surprise that in muscular diseases such as "Myasthenia Gravis" complete rest such as is given by a double Thomas splint seems never to be advised.

What Constitutes Muscle Rest.--A normal muscle cell is possessed of these two properties, the power of contraction and the power of relaxation. The stimulus which causes one set of muscles to contract causes those which act in an opposite direction to relax. To use a mechanical similethe pressure in the contracting muscle which is producing the volitional effect may be at 90 degrees, while that of the relaxing muscle may be only at 40. Either of these states, contraction of a muscle, or elongation of its relaxed opponent, might be described as an irritable state. The intermediate position, which is that of rest or equilibrium, is not a state of inaction, but is an active state in which the opponents are evenly balanced-the state in which, to apply the simile, the pressure in each group stands equal at 45 degrees. Thus, if we were resting the radio-ulnar joints at which the rotary movements of pronation and supination occur, the position midway between pronation and supination, with the pollex up and the minimus down, would be the position of repose. If both pronators and supinators were paralysed, the position of rest would be this normal position of equilibrium between the two groups. 
If a muscle be weakened or paralysed, whether from injury of muscle, nerve, or central cell, it is rested when its opponent is in a state of relaxation and elongation beyond the state normally regarded as necessary to produce a condition of equilibrium with its opponent. In this way we prevent a weakened muscle being stretched and irritated by the contraction of its opponent, and we assume that the application of electricity or massage is not allowed to interfere with recovery. Thus in paralysis of the supinators of the forearm the position of rest would be with the hand over-supinated, so as to prevent action of the normal opposing pronators. In the upper limb gravitational force has particularly to be remembered. If we leave our paralysed deltoid, as is usually done, unsupported with the hand hanging at the side, not only have we not true muscular rest, but have in addition the weight of the upper limb dragging on a weakened muscle. Frequently so great an eventual atrophy results that the glenoid cavity may be digitally explored. It is inconceivable that with all these disabilities massage should be advised.

Since rest is the basic treatment of inflammation, then only by the "zero" position - the position as defined abovecan the door be closed on all sources of irritation, and cord, nerve, "receptive substance,"* and muscle be at physiological rest. Furthermore, the "zero" position of rest is the position from which the muscle can most effectively commence work. When we ask a patient with a deltoid paralysed from poliomyelitis to commence work, as is usually done, when sitting up with the upper limb hanging at the side, we are asking damaged cells to begin work at the greatest disadvantage. asking a baby to walk before he has learned to stand. We must ask muscles to begin work at "zero," to commence work by beginning with a minimum function or at a point where their load is at a minimum. Though we speak of a muscle anatomically as being flexor or extensor we really refer to the maximum function of a muscle. The question arises as to the recognition of the minimum of muscular

* Existence of this substance in muscle is still theoretical. 
function, for it is only by the recognition of the minimum that the muscle may ultimately be coaxed up to the maximum-the ideal aimed at by our treatment. Thus, in reeducating the quadriceps we recognize that it is incorrect to flex the knee beyond the point at which extension is possible, at which effort becomes manifest-thus recognizing the cessation of the opposition of the quadriceps to its opponents. The treatment for the re-education of a paralysed muscle may be summed up as the encouragement of voluntary muscular movements within functional limitations. We begin at "zero," and although we may think that the amount of work at this minimum is slight, we must remember that it really represents the maximum function of the muscle for the time being, and as such may soon become exhausted. In the same way, the normal individual would soon tire if asked to continue flexion and extension at the elbow joint for some time, and to the full extent.

\section{Chronic Muscle Shortening: Contracted Muscle}

Contraction or chronic shortening of muscle is a secondary result of the unopposed volitional contraction of a muscle or group of muscles. The muscle cell has the double property of contraction and relaxation. Ordinarily, the volitional stimulus which. causes one muscle or group of muscles to contract causes also the opposing muscle or group to relax; and this relaxation permits of elongation by the contracting opponent. In other words, the pressure within or the force of the contracting muscle, is greater than that of the relaxing opponent. But if we suppose that as the result of inflammation of anterior horn cells, or injury of the nerve trunk, a muscle is incapable of carrying out its function, combustion is interfered with, whereby its pressure is lowered, and it is in the state of a muscle which has relaxed to allow its opponent to act. Equilibrium between the muscle and its opponent is upset, and if the normal muscular engine is not prevented it will be found that under some volitional stress it will pull on the damaged 
engine and cause its elongation ; in other words, the normal muscle will physiologically contract. But apart from volition this physiological contraction need not necessarily occur.

Thus, if we had a paralysis of the pronators of the forearm from division of the median nerve, occurring with the hand in the position midway between pronation and supination, apart from volition, aided in some cases by gravity, there is no reason why contracture should occur. There is no immediate rebound of the normal opponent producing contracture. But perhaps the patient brings the hand to the mouth, and supination is necessary. The supinated hand cannot be pronated, and unless passively restored to its old position it remains supinated. But perhaps for a fortnight, or longer, the supinators are not " contracted "- the hand can readily be passively restored. But supposing it remained supinated for six weeks, it may then be difficult, or even impossible, in spite of the patient's volition and outside passive help, to pronate the forearm-the normal and physiologically contracted supinating muscle has now become chronically shortened. But primarily the contracture of the unopposed muscle or group of muscles is physiological ; it is volitional: If in the above condition the pronators at the end of a week completely recovered, nothing pathological had occurred in the cells of the supinators to prevent their immediate relaxation and elongation by voluntary pronation.

Chronic shortening of muscle occurs most frequently in the flexors of the knee following paralysis of the quadriceps, in the pectoralis major following paralysis of the deltoid, and in the extensors of the ankle (tendo achillis) following paralysis of the flexors of the ankle. In these instances the gravitational effect of the weight of the leg, or of the upper limb or of the foot, would be a primary factor in producing contraction, in addition to the volitional contraction of the normal flexors, adductor, or extensors. Why the physiological contraction has become chronic is not due in my opinion to a further physiological or patho- 
logical change in the cells of the muscle, but to the correlated shortening of the fibro-elastic elements. These are present in varying degree in all muscles, and include the tendons of origin and insertion.

The chronically shortened or contracted muscle is the result of shortening of the tendon, and of the fibrous elements within the belly with which the tendons are directly continued. It is an adjustment or accommodation effect, and is seen in connection with paralysis of the extensors of the toes and of the tibialis anticus, where the deep fascia of the front of the leg shortens on the weakened irresistant muscles. Should recovery take place this may actually form a mechanical barrier to muscular action, unless divided or even partially removed by the surgeon. I have seen these muscles bulge out nearly half an inch when the deep fascia of the leg has been divided. This is why chronic shortening is seen especially in connection with the hamstrings and the muscles forming the tendo achillis. In the case of the deltoid the dragging weight of the upper limb-operating whether the patient is erect or lying down - is the paramount factor in producing pectoral contraction. It is interesting to note that in a young kangaroo (saltatorial marsupial) the total length of the tendo achillis and its muscles was $6 \frac{1}{2}$ inches-of this the tendinous length was 4 inches. Unfortunately shortening of the ligaments of a joint may also occur, and this must be remembered in connection with equinus of the foot, where the posterior ligament of the ankle may be so shortened in an old case as to need division. If we take, however, the treatment of a case of talipes equinus of three months' standing, we correct the foot by treating the tendon, either dividing it or lengthening it by operation.

Treatment is a purely mechanical question. Within ten days, when the lengthened tendon had sufficiently repaired, I have seen the tendo achillis muscles contracting normally, so as to elongate their relaxed opponents, although the former muscles had been chronically contracted. The state of "chronic contraction" had not interfered with 
the effectiveness of the muscle cell. From the physiological standpoint it was normal, only the muscle was mechanically prevented from being elongated either passively-even under an anæsthetic--or by the contraction, in cases of " paralysis," of a recovered opponent, although the cell itself had the normal power of relaxing and contracting. This can also be demonstrated with the patient lying down in the case of a pectoralis major which is " contracted," when the muscle will only allow of abduction of the arm to 60 degrees. There is obviously no interference with relaxation of the pectoralis muscle cell, since it allows the deltoid to elongate it to 60 degrees; nor is there interference with the contraction of the pectoralis cell, since it can bring the arm slowly and methodically to the side again. Similarly, with a case of contracted tendo achillis in which, though the foot cannot be dorsiflexed to the right angle, the muscles of the tendo achillis may contract to extend the foot 5 degrees further and relax to allow the foot to be flexed these 5 degrees.

Deformities of limbs produced by chronically contracted muscles acting against weakened opponents should never be seen. These are not necessary accompaniments of paralysis, as some would seem to imagine. They are avoidable. It should be impossible to see anywhere spastic claw hands, dropped wrists, dropped feet, or contracted knees or shoulders. Ankylosis of joints due to severe bony injury may be seen, but deformities due to muscle weakness should always be prevented. In cases of childien treated privately parental neglect in the application of splints must be borne in mind. If the above view be correct, it follows that the treatment of contracted muscle, should it have been allowed to occur, is simple and dependent on mechanical means.

To sum up as to causation, we find that as the result of volition the normal muscle is contracted. By volition it can also relax, but owing to the paralysis of its opponent no elongation follows. It remains permanently contracted owing to the correlated shortening of the fibrous elements 
of the muscle, which then prevent muscular elongation should recovery of the opponents occur. The indication is to elongate either by mechanical or surgical means the shortened fibrous elements, and this treatment is invariably successful. The chronically contracted muscle is not favourable to the recovery of the affected opponent, whose fibrous elements are thereby chronically stretched. This state of chronic elongation is one of irritability to, and not of rest for, the affected muscle cell. In treatment, then, the principle to be borne in mind is, that if a muscle be chronically contracted by shortening of its tendons and other fibrous elements, it will elongate if coaxed, but not if forced, and if the pressure, however slight, be continuous. In children especially, in whom contractures are most frequently met with, conservation rather than destruction should be our aim-the preservation of structures round a joint rather than their division.

Before division or lengthening of adductors, or hamstrings, or pectoralis, or biceps, a recourse should be had to concentrated energy, not so much forceful as continuous. " In animals," wrote Hunter, " there is more variety of motion, but in plants more real power. A small vine can raise a column of fluid five times higher than a horse can; for the same energy which in the animal world is weakened by being directed to many objects, is, in the vegetable world, strengthened by being concentrated on a few." Concentration of opposing force may be regarded as a first principle in the treatment of contractures. Given a pressure, however slight but continuous and concentrated, and contractuures even of the extreme and apparently hopeless type will be found to yield, without the aid of what should always be the last and not the first resort-the knife. And provided this principle be borne in mind it matters little by what means it is carried into effect. Thus I have known a badly contracted knee of some years' standing yield to the pressure continuously applied for three months of a bandage and a wooden back splint extending from the upper third of the thigh to the lower third of the leg. 
The breaking down of muscle contraction, like the "smashing up" of joint adhesions at one sitting under an anæsthetic, cannot be too vigorously condemned as being unscientific and charlatanic. It is an attempt to overcome in twenty minutes a state that has taken nature perhaps years to effect. By such a procedure permanent injury is usually done to the muscle. It has to be borne in mind that just as the fibrous elements of one muscle or group of muscles are shortened, so also are the similar elements of the opponents elongated. It takes time for these elongated fibres to tighten. The elongation of the fibres of the contracted group should be pari pass $u$ with the tightening of those of the elongated opponent. Then if the affected muscle has recovered, equilibrium will be restored between the elastic and the true muscular elements of the opposing groups. Until recovery takes place in the muscle cell of the weakened opponent there will always be a liability to return of contraction, unless this is mechanically prevented. In the case of the foot with a tightly contracted tendo achillis of old standing, it is usually impossible by ordinary mechanical force to overcome the deformity. Elongation of the tendon by surgical interference becomes necessary. It may be mentioned that contraction of the tendo achillis could be prevented and more frequently overcome in its early stage, if it were recognized that it is not sufficient merely to maintain a foot at right angles. Plaster of Paris from the ball of the toes to the middle of the leg is of little use. Extension of the knee is essential, as the plantaris and gastrocnemius, both of which terminate in the tendon, arise above that articulation.

Even should recovery of the flexors of the ankle have taken place, it will be necessary to keep the foot at rest for some time after operation to allow the elongated fibrous elements of the flexors to shorten. In other words, without support, the flexors, though recovered, may be unable for some time to hold the foot at a right angle. In such a case the patient when resting should have the foot in a back splint and a right-angled foot-piece; and when walking about 
should wear an extensor band passing from the dorsum of the boot to a metal wing applied at the middle of the front of the leg. Contractures associated with replacement of the muscle fibres by connective tissue, as seen in "ischæmic" paralysis following tight bandaging, or in connection with syphilitic myositis are probably the most difficult. When fleshy contracted muscles like the psoas, pectoralis major, or adductores, are not yielding to treatment-and this it may be stated is extremely rare-the presence of a luetic fibrosis might naturally be suspected.

\section{Xi. Testing for Muscle Action}

It cannot be too strongly insisted upon that there is only one true test for muscular action, the volitional test scientifically applied. It is the only test which takes into account the two elements of muscular action, the contraction of one muscle and the relaxation of its opponent. By the volitional test we are enabled to determine the minimum action from which the muscle can be re-educated up to the maximum. For the accurate application of this test, the ancestral history of the muscle must be considered. We must have a knowledge of the lines along which it acquired its maximum function, as in the case of the deltoid and the quadriceps. In addition, we must consider the effect of gravity; the placing of the origin and insertion of the muscle as nearly as possible on a level, as in the case of the brachialis; the avoidance of frictional effects by directing that the limb should be placed on a sheet of smooth cardboard; the position in which the "moment of force" of the muscle is greatest, as in the case of the flexor, pronators, or supinators of the forearm; also the position in which leverage can be most effective, as in testing for finger flexion, by asking the patient to first dorsi-flex at the wrist joint.

There should be complete normal range of movement of the components of a joint that constitutes the fulcrum for the affected muscle. Should the contraction of an antagonistic muscle have been allowed to occur through neglect, 
this should be first overcome, as often a muscle condemned as hopeless is really a recovered one mechanically prevented from acting. Tests that do not consider these principles can be dismissed as worthless. On these the prognosis should be based, and the opinion be expressed as to whether operative interference in a case of suspected nerve injury is necessary or not. Ignorance of these principles and failure to recognize the importance of the insertion of a muscle as an indication of its function, are at the root of the scepticism with which the demonstration of an early recovery of muscle, after nerve-muscle injury, is received. Results actually present are doubted, because they do not conform to traditional text-book opinion. It is interesting for the physiologist to explain, why after repair of a complete division of the median and of the ulnar nerves in the arm, when the muscles supplied by these two nerves were tested on the above lines, function, though small, was found in nine weeks to be present in every muscle supplied by these nerves. And why also, as the result of re-education, there was a complete recovery in that period of the flexors of the wrist and of the pronators of the forearm. We can test for recovery and commence to re-educate after nerve division as soon as the surgeon is satisfied that firm union has occurred. Graduated or pari passu re-education is not a source of irritation to a repairing nerve. To those not previously acquainted with these methods the early stage at which muscular movement will be noted will come as a revelation.

A few instances are worthy of mention as to the method by which muscles are usually tested for action, and the unscientific grounds on which they have been condemned as hopeless because they have apparently failed to respond to massage and electric treatment. In the case of the flexor of the forearm it is usual for the patient to be asked to bring the hand up to the mouth from the hanging down position of the arm, or to do the maximum work for that muscle of roo units. Here the weakened muscle is asked to bend the forearm with the hand against gravity, 
with its line of action lying parallel with the long axis of the extremity, and its " moment of force" being at a minimum. The patient, on the contrary, should be lying down on a firm bed or table with the limb resting on a firm pillow, the elbow should be passively bent to a right angle -the position in which the " moment of force" is greatest -and any movement should take place on a sheet of powdered cardboard to eliminate all frictional effect.

In a case of deltoid paralysis the patient is invariably asked to elevate the arm from the side when sitting or standing erect. Yet a patient who fails in this maximum effort may readily, when lying flat, abduct the arm to a right angle. How often do we find a quadriceps regarded as " paralysed," because the patient is unable when sitting to lift the heel off the ground with the knee extended! Yet such a quadriceps, if tested with the patient lying on a firm bed, may, extend an acutely flexed knee. The pronator teres should not be tested with the patient sitting erect or standing. He should be lying down with the arm resting on a pillow, the forearm at a right angle and midway between pronation and supination. To test the inverting or everting power of the tibialis anticus or peroneus brevis, and the inverting or everting power of the tibialis posticus or peroneus longus, the foot should be dorsi-flexed in the case of the two former and extended (plantar-flexed) in the case of the two latter.

\section{Correlations}

At the foundation of all study on function lies not merely a knowledge of structure but, what is more important still, a knowledge of correlated structure; and this constitutes what is meant by applied comparative anatomy. The study of structural correlations throws numerous hints, not merely on normal, but also on so called abnormal or monstrous structures. Changes regarded as monstrous in us may be seen in other animals to be correlated to other changes; in other words, nature has in different members of the mamma- 
lian class carried certain of her adaptive trends farther than with us, giving us an indication of the intended procedure in man. Thus light is thrown on the mode of disappearance of the fibula, the possible significance of a cervical rib, mode of disappearance of the vermiform appendix, significance of the large gut in the economy, and on the functional value of certain muscles. When we speak of "highest" and "lowest" amongst the mammalia we refer really to the relative development of the new brain. The comparison relates to cerebral and not to physical development. Thus, if we take the case of the vermiform appendix, we find that in the wombat (marsupialia) this structure has evolved farther towards complete disappearance than in man. By a study of the abdominal cavities of the koala and phalanger light is thrown on the vital importance or otherwise of the adrenal glands and on their mode of disappearance. When we hear it stated that the large gut is practically a useless structure, and that the ideal gut is a simple tube, it is as well to reflect that, in a member of the marsupialia, namely, the Tasmanian devil, such a canal has been evolved. A study of correlations, however, shows this animal to be the most defective muscular type of its order. If nature producesand apparently she is doing it frequently-a new rib in the neck, the question pertinently arises, as also in the case of the disappearance, partial or complete, of a bone usually present, for example the fibula, is this disappearance or appearance utilitarian and in response to a need ? In other words, are there evidences of correlation of the rib with associated structures indicative of permanency in the effort? If symptoms present themselves, are these due to a failure in the correlating process either natural or mechanically produced ? This must be the test applied to all so called abnormalities.

Nothing in itself should be regarded as abnormal or monstrous; the variation may be a useless one, nevertheless it is a response to an effect or need. The removal of the rib can only be scientifically justifiable when it is proved that removal is the factor necessary to secure adequate correlations of the adjacent structures. The fact 
that complete correlation could occur was demonstrated in Turner's celebrated dissection of a cervical rib extending to the first thoracic costal cartilage, in which a complete adjustment of parts could be demonstrated. Similarly there is abundant evidence that the human fibula is undergoing adaptive changes. In man, so called " congenital fractures" are met with in this bone, or even its com-

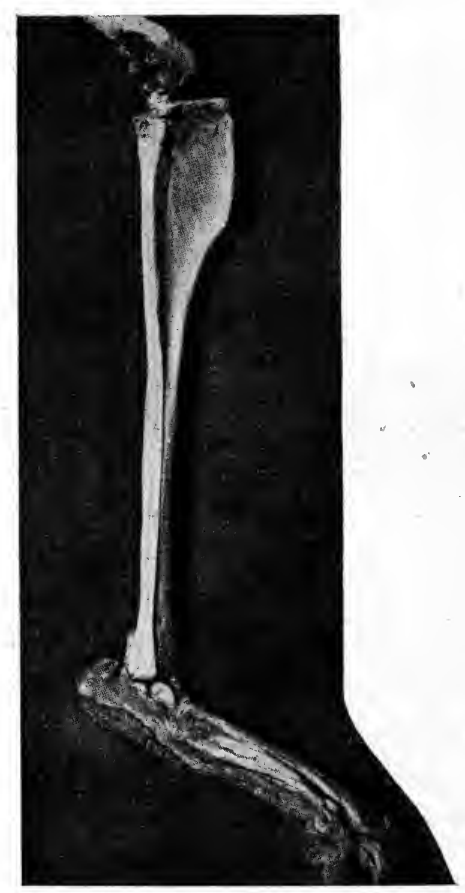

FIG. 5.-The leg and foot of kangaroo.

plete disappearance with loss of the outer toes. Such cases may serve as an indication of ultimate adaptation, although in causing a congenital absence of the human fibula to occur, nature has carried the progress of one of the consequences of the erect position and associated gait beyond that of the other consequences with which it should be co-ordinate. It is not enough to say that the fibula is disappearing, unless one finds correlative evidence in the toes 
and muscles of the leg and foot. The leg of the kangaroo, in which the lower half of the fibula does not exist separately from the tibia (fig. 5), throws important light on the natural mode of disappearance of the fibula. Here muscle correlations can be studied, and also the method of correction of the

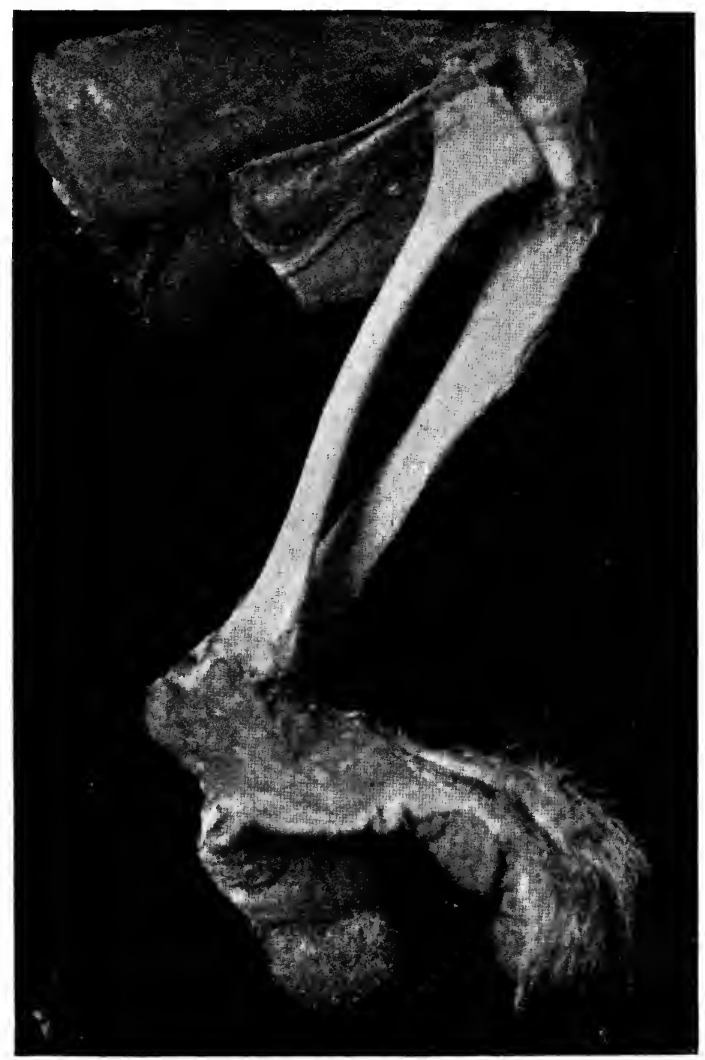

FIG. 6.-The leg and foot of koala (Phascolarctos cinereus).

error. In the koala the relatively large fibula is associated with an opposable hallux (fig. 6), and in this animal and in the phalanger there exists a greater freedom of movement between the tibia and fibula than in others of the marsupialia. This approaches in some degree the rotation between the radius and the ulna 
On similar lines one is able to determine the "survival value" in man of a muscle such as the brachio-radialis (supinator longus). From the variety of functions ascribed to this muscle one infers that it is regarded by many as almost the most important in the upper extremity. To it are ascribed the triple powers of pronation, of supination, and of flexion of the forearm. Those who ascribe the power of elbow flexion to this muscle seem to forget that this would involve the relaxation of the antagonistic and powerful triceps, which is only associated with brachialis contraction. This muscle (brachio-radialis) is an accessory supinator. Its insertion is on a bone whose muscles permit of rotatory movements only, and not the forward and backward movements of flexion and extension. Its maximum development is reached in the wombat, which is the most powerful muscular animal amongst the marsupials. In this animal it extends above as high as the spine of the scapula, and has a broad attachment below. The wombat is a burrowing animal, and for this powerful pronation and supination are necessary; the hand has an unopposable pollex. In the koala this muscle is also well defined, and at its termination it is seen to cross dorsal to the radio-carpal articulation to terminate at the lateral side of the carpus. Its development in this tree-climbing marsupial is associated with a double pollex by which limb grasping is improved. In man the supinator longus has a narrow tendinous insertion on the lower end of the radius, and in the kangaroo there is little muscular belly, the greater part of the muscle being a slender tendon which is attached more proximal and more extensively to the radius than in man. This muscle in the human upper limb can be regarded not as one possessing a multiplicity of function, but as one struggling hard to retain its place in the human economy.

Muscle Development and the Thymus Gland.-The thymus gland is a characteristic of early human life; it commences to disappear about the fifth year, and in the adult nothing is to be seen macroscopically. At birth it weighs $\frac{1}{2} \mathrm{oz}$. or more. At twenty-one it is usually a remnant 
weighing only forty grains, and after twenty-five it is extremely difficult to discover any of its structure in the mediastinal tissues. Cases of a defined human thymus being retained throughout life are recorded, a fact of no little importance. The thymus gland is at its maximum development when muscular action and co-ordination associated with the erect posture are developing; these being well established it commences to disappear. In the recorded instances of its persistence throughout life its presence has presumably been a necessity, and harmless. Although it commences to disappear early, the question arises, should it necessarily disappear then ? Is its absence beneficial in all or only in some? Are conditions superimposed causing its too early disappearance in some to whom perhaps a thymic secretion may be a necessity? On these questions a study of muscular and thymic correlations throws some interesting light.

In the platypus at one end of the mammalian scale, in which piscatorial, avian, and mammalian features indicative of instability are to be found, the thymus, apart from any question of hibernation, is present throughout life. I have found it covering the ventral and lateral surfaces of the pericardium with a thickness of nearly $\mathrm{I} / 8$ inch. In the Tasmanian devil (Dasyurus sarcophilus) also, a well developed thymus is retained throughout life. This exclusively carnivorous marsupial has during the past century offered a poor resistance to man, and like the Tasmanian wolf (Thylacinus) is now almost extinct. The dasyures or carnivorous marsupials represent from the point of view of musculature the poorest developed type amongst marsupials. There would appear to be a definite relationship between this and the retained thymus. If, on the other hand, we were asked to designate the perfected physical type from the point of view of musculature amongst marsupials, one would unhesitatingly refer to the wombat. Unprotected by law, it has easily offered the greatest resistance to the presence of man on the Australian continent. Yet in this animal I have never met with the presence of thymic tissue either in the young or in 
the adult. I have directed attention to this question as possibly throwing light, not so much on the treatment as on the causation of certain muscular and nerve diseases. I would suggest the use of thymus gland in the early stages of poliomyelitis during re-education, and similarly during the re-education of weakened muscle from nerve or other injury. The question is suggested whether in some remote way this gland is associated with the failure in man of a limb to regenerate after amputation-a power which our remote ancestors before the evolution of the mammalian type appear to have possessed in conjunction with a highly developed reflex system.

XiII. Contraction, Relaxation, and Elongation of MUSCle: Elasticity

When we use the term "muscular action" we are not referring to the síngle action of muscular contraction, but to a double physiological action, namely contraction and shortening of the muscle producing the desired effect at the fulcrum or centre of motion, since the origin and insertion are approximated; and relaxation and elongation of its opponent. The voluntary stimulus causing one muscle or set of muscles to contract causes also relaxation of the opposing muscle or set of muscles. Just as important as a knowledge of the action of a muscle is a knowledge of the action of its opponent. A muscle fibre cannot be in a state of physiological relaxation and physiological contraction at one and the same time. Contraction and relaxation of muscle are two opposite physiological states. Relaxation does not mean, as some have supposed, a cessation in function of the muscle fibre. It is as much a physiological action as contraction; and it is to be remembered that muscles which contract at death do not relax post-mortem. Normally, muscles in life maintain a constant action which is known as " tone," but neither contraction nor relaxation of muscles is produced after death. In a normal muscle during life there is no such thing as a state of absolute rest or inaction, so that in connection with fixation of muscle, 
for example in bone or joint injury or disease, we can never produce absolute rest of normal muscle. This can only be produced by death. Elongation of muscle, on the other hand, is not a physiological action, but is produced as the result of a mechanical action. Whether we are dealing with voluntary or involuntary muscle, elongation

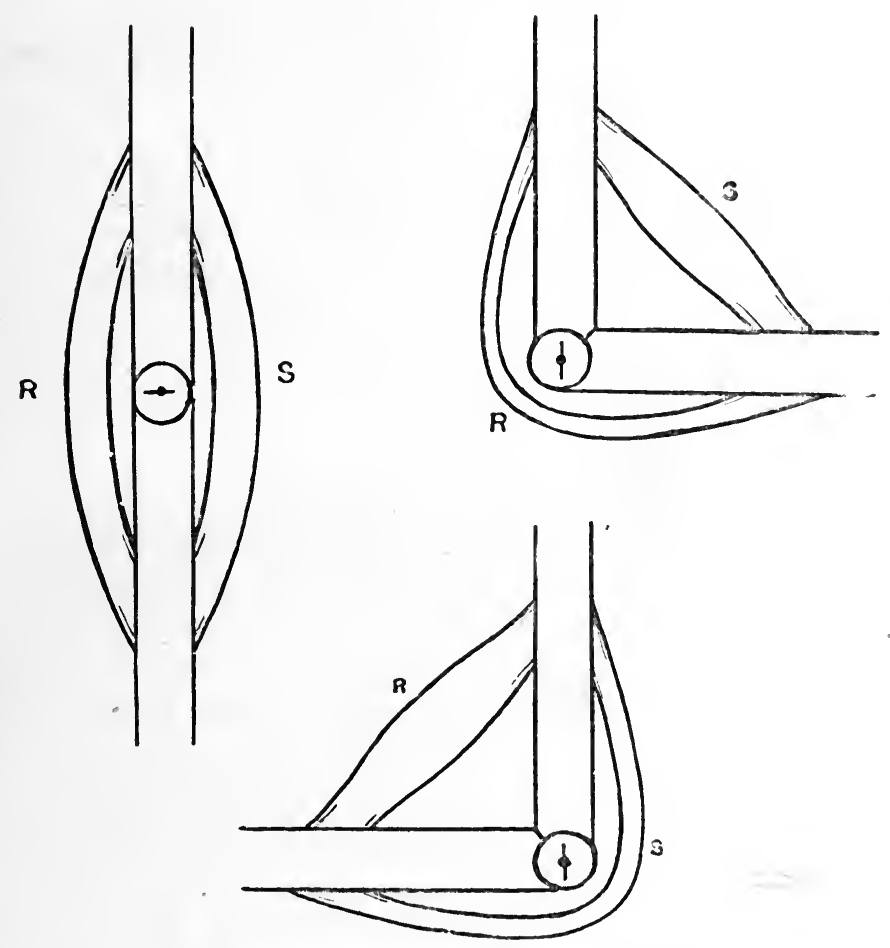

FIG. 7.-Diagrammatic representation of muscular action, and reciprocal elongation.

depends for its production on some outside force. The power of elongation is not inherent in the muscle fibre.

Relaxation is essential for elongation, it must precede it ; but there is no inherent force in a relaxed fibre that produces elongation. In the case of voluntary muscle the relaxed muscle $(R)$ is elongated, owing to the contraction and shortening of its opponent (S). When the relaxed 
muscle fibre $(\mathrm{R})$ in its turn contracts, the opponent (S) simultaneously relaxes, and is itself in turn elongated (fig. 7). Thus the opposing muscles or groups of muscles throughout the body become in turn reciprocal elongators of each other. In the case of involuntary muscle action, as in the propulsion of food along the gastro-intestine, elongation is produced by the mechanical impaction of food. The presence of food in a suitable state of digestion is the stimulus to contraction of that portion of the gastro-intestine with which it is in contact-the successive portion of intestine becomes relaxed to allow of the propulsion of food by which it is elongated, and this portion in its turn contracts, propelling the food into the adjacent distal relaxed portion.

The action of an elastic body is, on the other hand, of an essentially different nature from that of a muscular one. An elastic body can produce movement by shortening, after it has been stretched, just as muscle can ; but two opposing pieces of elastic tissue, unlike two opposing pieces of muscular tissue, cannot reciprocally elongate each other. The muscle fibre has the power in itself of producing motion; it can relax and contract. An elastic fibre has no inherent power of contraction or relaxation, and it is dependent on an outside power which is not elastic for its elongation. By this elongation it is enabled to produce motion on a part, since it is always striving to arrive at the normal state in which it is not stretched, and this it does as soon as the stretching force is removed. Unlike muscular action the property of elasticity is not affected by death. We can inject and dilate blood vessels after death, or stretch a tendon which shortens when the stretching force is removed. By means of their elastic property the ligaments of a joint are enabled to accommodate themselves to alterations in the angles of the components of the articulation, which alterations cannot be produced by ligaments, but by muscle. The ligaments, when stretched, are factors which are always endeavouring to approximate the angle of the components of a joint to the normal. The use of elastic substance in muscle is in connection with the state of equilibrium between 
two opposing muscles or opposing groups of muscles. The elastic components would be constantly striving to produce the state of equilibrium, and indeed this may also be said of the muscle fibres. When a contracted muscle (S) (fig. 7) relaxes, its elongation towards the middle state of equilibrium is the result as much of the recoil of the elastic substance of the contracting muscle (R) as of the action of the contracting muscle itself ( $R$ ) which had been elongated. Beyond that, action would appear to be solely muscular. The state of equilibrium is the state when muscles or groups of muscles are equally balanced, not only as regards pressure or tension in the muscle fibres, but also when the opposing elastic fibres are themselves in a state of equilibrium. When two opposing muscles or opposing groups of muscles are paralysed, the position of rest corresponds to the normal position of equilibrium between the two groups. A muscle which is elongated during normal muscular action presents a firm, tightened feel to the touch, which is often and erroneously regarded as a state of contraction. Thus, when we stand erect, although the hamstrings feel firm, they are in a state of relaxation and elongation owing to the contraction of the quadriceps by which the erect position is maintained. If we bend the body back at the knee, the flexors of the knee contract and the quadriceps relaxes and elongates, but this latter is pari passu with the contraction of the hamstrings. In other words, flexion is not an unregulated action of the flexors ; by means of the extensors a too forcible action of the benders is prevented. Relaxation of muscle is thus essentially an active, not a passive state; the relaxation of the opponent goes hand in hand with the action of the contracting muscle. A good example of the evil attendant on the uneven balance between elongation and contraction of opponents is seen when a person in danger of falling backwards makes a sudden muscular effort to prevent this. This effort causes a spasmodic contraction of the quadriceps without pari passu relaxation and elongation of the flexors, and may result in a yielding either of the patella itself or of the ligamentum patella.

When I use the term "centre of motion or fulcrum" in 
reference to the action of muscles or groups of muscles I do not refer to the centre of motion of the body, which would appear to be at the hip joints; but to the particular joint, the angles of whose components are altered by the muscle or muscles when they produce motion. When I use the term "muscular opponents of a joint" I refer to muscles or groups of muscles which have the power of reciprocally contracting and elongating about the same centre of motion, and not to muscles which are merely passing over the particular centre of motion to act on some distant articulation. Thus, if we take the case of weakened flexor action of the second and third phalanges of the fingers (sublimis and profundus), we find that the patient will invariably dorsiflex the wrist to obtain the benefit of good leverage. This he does by contracting one and reciprocally relaxing the other of the two opposing groups of muscles acting on the same centre of motion at the wrist joint.

The stimulus to contraction of the extensores radialis longus and brevis and the carpi ulnaris calls into physiological relaxation the flexores carpi radialis and ulnaris, by means of which the contracting extensors are enabled mechanically to elongate them. With this motion the muscles acting on the fingers have absolutely no connection. We can produce this position of the wrist with the fingers either flexed or extended, abducted or adducted. The long flexors of the fingers, though passing over the wrist, do not act on it: their contraction does not call into relaxation the extensors of the wrist without which flexion of the wrist is not possible. Their action calls into relaxation the extensors of the two distal inter-phalangeal articulations on which they act, namely the interossei. Similarly, the extensor communis which produces extension at the metacarpo-phalangeal joints of the fingers does not extend the wrist. The same stiniulus that produces its contraction does not cause relaxation of the flexors of the wrist without which extension is impossible. On the contrary, it produces relaxation of the muscles which act on the same joints, those which have the same centre of motion or 
fulcrum, namely the lumbricales, and which are the benders of the metacarpo-phalangeal joints of the fingers.

In the case of the elbow the muscles which have the same centre of motion, the humero-ulnar joint, are the brachialis in front and the triceps behind. The stimulus necessary to produce contraction in the one simultaneously produces relaxation in the other. By means of these muscles, and these only, flexion and extension of the elbow joint are produced. The biceps brachii is a supinator. The stimulus that produces its contraction causes pronator relaxation, without which its action is impossible. Its centre of motion is radio-ulnar and not humero-ulnar. This stimulus does not produce relaxation also of the powerful triceps, without which elbow flexion is impossible. The brachialis is the only muscle whose stimulus to contraction has that effect. The supinator longus (brachioradialis) is often erroneously described as a flexor of the elbow. It has no such power. It is attached to the radius, which has a rotatory motion (pronation and supination). The centre of motion for the action of the long supinator is radio-ulnar, and the stimulus for its contraction does not simultaneously call into relaxation the powerful extending triceps without which elbow flexion is impossible. It may be argued that if a movable elbow joint be improvised with the bones of the upper limb, and if straps be attached corresponding to the insertion of the biceps or supinator longus, traction on these straps will produce flexion of the elbow joint, and hence these muscles must be elbow flexors. That argument holds if the question of triceps action be excluded. It will not hold if, for example, a splint be fixed dorsal to the elbow joint so as to prevent its motion, for that is practically what the contracted triceps corresponds to as regards the supinator longus and biceps in a normal upper extremity.

\section{Reverse Action}

In " reverse action" there is an alteration of the terms applied to the termination of the muscles. What is usually 
regarded as the origin of the muscle becomes the insertion, and what is usually regarded as the insertion becomes the origin. Thus in the case of the brachialis the insertion into the ulna becomes the origin, and its attachment to the humerus or origin becomes the insertion. The reverse action is seen when we flex the body on the fixed forearm and hand, as in elevating the body from the ground on the horizontal bar (fig. 26). Here we are dealing with a joint (humero-ulnar) admitting of flexion and extension only, and acted on by muscles (brachialis and triceps) which pass over it only. So that whether we are bending or extending with either muscular extremity - the arm or the forearm-as the origin or insertion the physiological action is the same. The contraction of one muscle causes relaxation of its opponent acting on the same centre of motion. In the case of reverse action of a muscle passing over two joints, as for example the sartorius or the gastrocnemius, it is the knee joint in the one case and the ankle in the other which constitute the centre of motion respectively for these muscles, whether the action be what is called "normal" or "reverse." Thus it is usually stated that the gastrocnemius with its fixed point below acts as a flexor of the femur on the tibia ; this implies that contraction of the gastrocnemius can call into physiological relaxation the extensor of the knee joint, the quadriceps. That muscle is antagonized however by the muscles which have the same centre of motion or fulcrum, namely the flexors of the knee. They alone can call the quadriceps into a physiological state of relaxation. The muscles acting on the ankle, and having the same centre of motion as the gastrocnemius, are the flexors of the ankle. It is their relaxation which permits of the action of the gastrocnemius, and the result of the reversed action of the gastrocnemius and the soleus is a limited extension backward of the whole body at the ankle joint.

Taking its fixed point from below, the sartorius is described as a flexor of the pelvis on the thigh. The centre of motion for the sartorius is the knee joint. If the knee be fixed in the extended position, as when we stand, it seems to be 
forgotten that the quadriceps extensor has contracted to produce this because all the flexors of the knee including the sartorius are relaxed and elongated. Furthermore, contraction of the sartorius does not call into physiological relaxation the extensor of the hip joint, the gluteus maximus, without which flexion of the pelvis on the femur at the hip joint is impossible. The gluteus maximus has its own reciprocal elongator, the ilio-psoas or flexor of the hip, and contraction of the ilio-psoas alone is accompanied by physiological relaxation of the gluteus maximus.

XV. Muscles passing over Several Joints Muscles passing over several joints, as the biceps (shoulder, elbow, and radio-ulnar) or the long flexors and extensors of the fingers, are with their opponents in a state of equilibrium as regards all the movements of the joints over which they pass. They are only concerned with their own particular function, and in the case of the flexor profundus digitorum this is an action on the last phalanx of the fingers resulting in an alteration of the relations of the components of the distal interphalangeal joints. The profundus is not a flexor of the elbow, wrist, or metacarpo-phalangeal joints. We can flex the distal phalanx of the fingers in the position either of flexion or extension of these joints.

The power that muscles have of adapting themselves to alterations which take place in the natural distance between their origin and insertion, as a result of action on the joints over which they pass by the particular muscles of those joints, would appear to depend on their elastic components. Thus when we flex the second and third phalanges of the fingers with a flexed wrist, and then extend the wrist still keeping our phalanges bent, this does not mean an alteration in the physiological state of the muscle fibre or cell of the profundus and sublimis, which remains one of contraction. The centres of motion for these muscles are the interphalangeal articulations, and only actual alterations of the angle of the centre of motion would be associated with alteration of the physiological state of the muscle cell. Again, if 
with the forearm at a right angle to the arm we over-supinate, this is due to the action of the biceps assisted by the supinatores, and also to accompanying relaxation of the pronatores. If we further flex the elbow, which is due to brachialis contraction and triceps relaxation, some slight shortening of the biceps will be noted; this is due to the elastic components of the biceps accommodating themselves to the altered angle of the elbow joint. It is not due to a physiological contraction of the biceps acting as a bender of the elbow as is erroneously described, since, with the elbow joint flexed from a right angle to 45 degrees by the brachialis, we can relax the biceps by over-pronation of the forearm.

\section{Specialization of Muscle Function}

Each muscle can be regarded as a specialist as regards function, and should several functions be ascribed to a muscle these can only be regarded as the result of one contraction. A muscle cannot be at one time an extensor and at another a flexor as is described in the case of the interossei of the hand. These muscles are supposed by some to have the dual power of flexing the metacarpo-phalangeal joints and of extending the interphalangeal ones. This however is not so, since, if we flex the two interphalangeal joints, which we do by contracting the profundus and sublimis, the interossei, as the extensors of these joints, must be in a physiological state of relaxation. Yet holding the interphalangeal joints flexed we can flex also at the metacarpo-phalangeal joints. This we do by contraction of the lumbricales and relaxation of the extensor communis.

Under normal conditions of volitional control, the two states of contraction and relaxation cannot be considered as existing in the one muscle at the same time. In spasm, which is abnormal, an irregular action of muscle fibres may occur. Similarly a portion of a muscle may be artificially stimulated, or the normal action may be interfered with owing to trauma of portion of the nerve supply or portion of the muscle. Speaking generally however, when the whole 
muscle acts it produces one general effect, having one defined action; and its combined force acts in the line of the resultant of the forces exerted by its component fibres. This combined action is only possible on account of the nervous mechanism by which the numerous fibrils composing the muscle are connected. The deltoid muscle, though it has an extensive origin, has a comparatively narrow insertion-the fibres converge towards a narrow area to produce a combined action. We see it stated that " the anterior fibres of the deltoid assisted by the pectoralis major draw the arm forwards, and the posterior assisted by the teres major and latissimus dorsi draw it backwards." This means physiologically that if the anterior fibres draw the humerus forwards they are contracted and shortened, and the posterior fibres which draw the humerus backwards are in this movement relaxed and elongated, two opposite physiological states existing in a muscle at one and the same time. This statement of deltoid action is erroneous, and leads, in astudent's mind, to endless confusion. The insertion of the deltoid is our guide for function. It should be regarded simply as an abductor of the arm and its physiological opponent is the pectoralis major.

\section{Synergists}

In any muscular action throughout the body there are numerous other muscles at work-synergists-whose action though secondary is designed to help the principal movement. Thus in lifting a weight above the head not only the muscles of the upper extremity are at work, but the muscles of the trunk and of the lower limbs, which maintain the erect position, an essential for this action, also participate. In picking up an object from the table when sitting, it is not only the opposed action of the thumb to the fingers; but the forearm, elbow, and shoulder muscles are also brought into action. The essential action however is the opposition of thumb to the fingers, and although we make a routine of certain combinations, these do not warrant the stress that has been laid upon them. Thus we can pick an object off 
the table with the wrist or elbow flexed or extended, and the arm abducted or adducted at the shoulder. We can abduct the little finger with the wrist abducted or adducted, flexed or extended, or midway between flexion and. extension.

What the student should particularly remember is that the components of combined muscular movements are muscles or groups of muscles in a state of either relaxation and elongation, or contraction and shortening, and acting at different but specific centres of motion. Thus in abduction of the little finger with the elbow flexed and the wrist dorsi-flexed we have at the elbow contraction of the brachialis and relaxation of the triceps ; at the wrist relaxation of the wrist flexors and contraction of the wrist extensors. The essential movement of abduction of the little finger due to contraction of the abductor minimi digiti is possible owing to adductor relaxation, and to that only. This action takes place at the metacarpo-phalangeal joint of the little finger, which is the centre of motion or fulcrum, and is independent of the action of muscles which have not that articulation as their fulcrum or centre of motion.

\section{Mechanism of Muscular Contraction}

As to the nature of the mechanism of muscular action whether it can be explained by physical or chemical methods, we must still confess our ignorance. But the ignorance of cause does not prevent us from investigating the principles by which muscular action is regulated, or from studying the effects of action. Charles Bell regarded muscle contractibility as an original endowment of nature, imparted in a way that could not be known. Older anatomists regarded the muscle fibre as a hollow tube from end to end; or a chain of cells of various shapes, some rhomboidal, some circular, some cylindrical, which contained foreign matter capable of being acted on. Following Newton's great discoveries the nerves and muscles were regarded by others as containing "the rare elastic spirit" which he concluded to be diffused throughout the universe. The effect of mental effort was to 
communicate a pulsation to this elastic spirit in the nerve, and so to the same spirit within the juices wherewith the muscle cells were filled, and the result was a rarefication and dilatation of the juices. When the mind ceased to act the impulses propagated in the elastic spirit ceased, and the muscular fluid, whose expansion was occasioned by these pulsations, subsided, and so relaxation was accounted for:

In recent times perhaps the most important view put forward is that of Professor MacDougall, "that osmosis might occur between the interior of the fibril and the sarcoplasm outside." It seems certain that with contraction of a muscle lactic acid is evolved, and the contracted muscle as compared with its relaxed and elongated opponent is in a state of increased tension. Oxidation is the accompaniment of relaxation or lowered tension, not of contraction or increased tension, and with relaxation of fibre lactic acid is absent. To resort to a mechanical simile muscle fibres always have steam up. They are always ready for action, and as stated a muscle can only be completely rested when this "irritability" " tone " or " inherent power" has gone, i.e. post-mortem. This power on the part of the muscle fibre of keeping up steam or tension corresponds to the power of the hepatic cells to secrete bile, or the pancreatic cells to secrete pancreatic juice. Its function has disappeared when it loses that power, just as the function of the pancreatic cell has gone when it is unable to secrete pancreatic juice. As an engine with a pressure of $80 \mathrm{lbs}$. will move an engine pulling in the opposite direction with a pressure of $40 \mathrm{lbs}$., so will the contracting muscle whose pressure is greater, pull and elongate the opposing or relaxed muscle whose pressure is less. In the state of equilibrium between two physiologically opposing muscles or groups of muscles the tension or pressure in the opposing muscle fibres is of equal intensity. Under similar conditions the two engines, each with $40 \mathrm{lbs}$. pressure, are unable to affect one another. What corresponds in the muscle fibre to steam in the engine is still unsolved. The explanation of this problem might almost be regarded as the explanation of life itself, for after all life is motion. 


\section{CHAPTER II}

\section{THE SHOULDER REGION}

THE upper limb of man with its fine intrinsic and extrinsic adjustments can, in comparison with the lower limb, be regarded as correlated to intellectuality. The main function of the lower limb is support, and its principal movements are the forward and backward ones of flexion and extension. It is more important to prevent ankylosis in the upper than in the lower extremity, since in spite of fixation of the three main lower limb joints a patient may walk comparatively well. In man so fine are the muscle adjustments in the upper limb that the loss of function, for example, of the adductor or opponens pollicis interferes at once with the opposition of thumb to fingers, an essential movement for grasping. Of the pronator teres, in which case food when placed in the hand can be conveyed to the mouth, but owing to the fixed oversupinated position of the hand cannot originally be grasped. Of the supinators, in which example food can be grasped, but owing to the hand being fixed in the position of overpronation cannot be conveyed to the mouth. The loss of any of these functions may be sufficient to ruin for practical purposes the utility of the limb-a condition of affairs that would never occur in the case of the lower extremity.

It is interesting to note that poliomyelitis affects the upper limb much less frequently than the lower, and adult affection of the former is extremely rare. If cases of upper limb paralysis from this disease are treated early and effectively on lines which recognize the ancestral sequence of acquisition of function, recovery is much more rapid and complete in the upper than in the lower limb-a result 
quite opposed to former statistics. Nor is the reason hard to seek. More perfect rest is secured, volitional control of muscular action is better, and the easier working from the "zero" position renders the muscles of the forearm and hand more responsive than those of the leg and foot. It might at first appear somewhat strange to state that there could possibly be any association between two marsupial

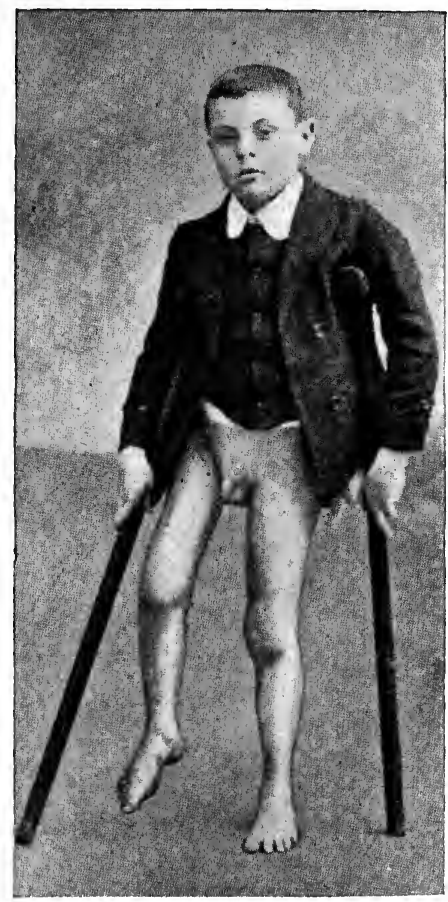

FIG. 8.-Quadriceps extensor paralysis (case of infantile paralysis).

animals - the wombat and koala-representing life on this planet many ages ago, and a soldier from the recent French battlefields who has been wounded in the shoulder region with resulting inability to raise his arm above his head. In this connection it is first necessary to define particularly two very important characteristics of man's erect or orthograde posture.

(I) That the quadriceps muscle produces the essential 
stiffening of the knee which enables the erect standing attitude to be maintained; failure to effect this is the cause of the swinging limb and crutchdom met with so often in old untreated cases of infantile paralysis (fig. 8).

(2) That not only has the upper limb been dispensed with

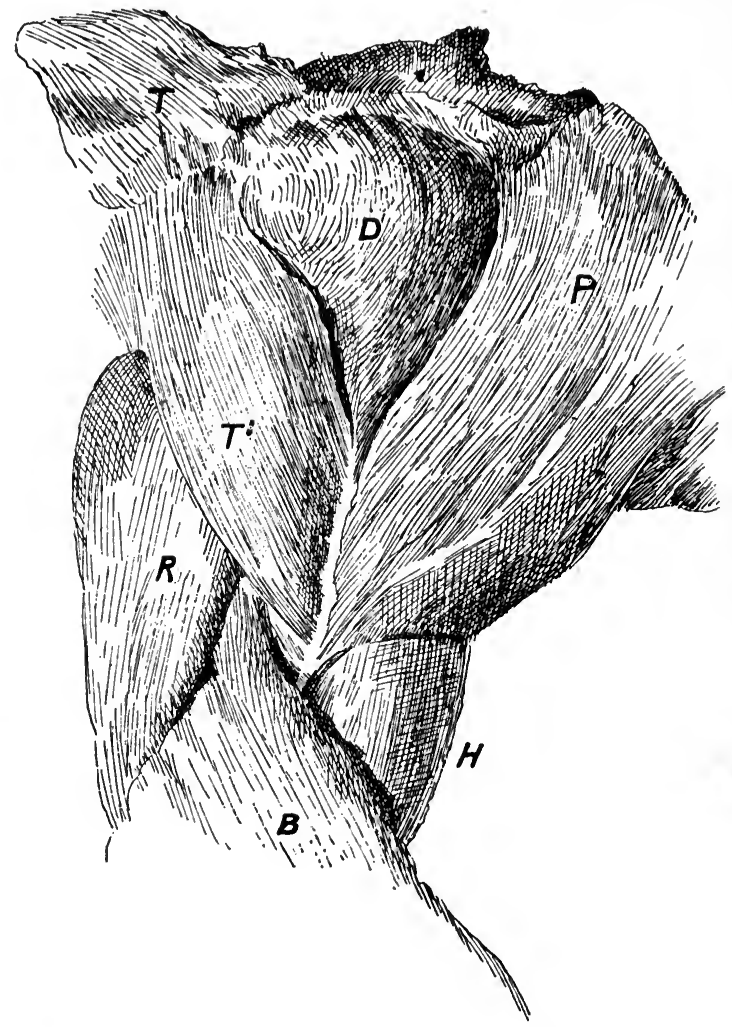

FIG. 9.-The deltoid region of koala.

$D$, Deltoid ; $P$, Pectoralis major ; $H$, Biceps ; $B$, Brachio-radialis ; $R$, Triceps ; $T^{\prime}$, Deltoid; $T$, Trapezius.

as a means of bodily support in progression, but, although dependent, it can be raised above the head.

These are the latest muscular functions to be acquired by man, for even such closely related mammals as the orang and chimpanzee walk with the aid of the fore limb, and the 
$\therefore 7$ 
knee slightly bent. Yet nature experimented in this direction when the marsupial was the highest form of mammalian life on the earth. The selective factor was in all probability drought. It drove the wombat below the surface of the ground for water and roots. It drove the koala to the trees for the eucalyptus leaf. Incidentally a vermiform appendix developed in the former, and a cæcum sometimes $8 \mathrm{ft}$. long in the latter. In the case of the koala (fig. 9) the ability to raise the arm was not associated with orthograde posture. The erect posture and the ability to raise the hand above the head were not correlated in the marsupials. There was a partial success in the kangaroo. The perfected correlation in man occurred many years later. In effecting elevation of the hand from the hanging position to above the head, nature was not prodigal in muscle expenditure. The keynote to the movement of elevation of the upper extremity is to be found in the characteristic development of the deltoid muscle as seen in man-deltoid development at the expense of trapezius and pectoralis major. The trapezius instead of extending to the humerus with the pectoralis, as is seen in the wombat, became limited by the spine of the scapula, the acromion process, and the clavicle (fig. Io). For the study of this important evolution the koala and kangaroo (marsupialia) afford abundant evidence. The action of the supraspinatus as an abductor of the arm is subsidiary to that of the deltoid, and would appear to be associated with external rotation of the humerus. It is important to notice that its development, unlike that of the deltoid, is not associated with the acquisition of the power of elevation of the fore limb. In an animal like the wombat in which there is neither attempt at the erect posture nor elevation of the fore limb the supraspinatus is a well-developed muscle. The deltoid in man is a lever of the third degree, the power is between the weight to be raised and the fulcrum or centre of motion at the shoulder joint. This ensures velocity. As evidence of strength, though it has an extensive origin it is concentrater at its insertion into the middle of the outer surface of the shaft 
of the humerus which is at some distance from the centre of motion. It is a fleshy muscle, the fibres being strengthened by fibrous septa to improve attachment. The arrangement of the anterior and posterior fibres is linear and that of the central ones bipennate. Once able to maintain the abducted arm in relation to the acromion by means of the deltoid assisted by the supraspinatus, nature found that all that was necessary for further elevation was a rotatory movement of the scapula. No new muscles were called into being. She utilized the rhomboids, trapezius, serratus, and levator anguli scapulæ by giving them new rotatory functions ; and as the abduction and rotatory powers are recent so also are they unstable, easily lost, and regained in cases of injury or disease with difficulty.

\section{MUSCLES OF THE SHOULDER REGION IN MAN}

It is necessary to distinguish between two groups, viz. :

(I) Muscles arising chiefly from the scapula to move the humerus;

(2) Muscles inserted into the scapula to produce the movements peculiar to that bone.

\section{Muscles moving the Humerus}

All these with the exception of the pectoralis major and latissimus dorsi arise from the scapula.

They are:

Abductors.-Deltoid and supraspinatus.

Adductor.-Pectoralis major.

External rotators.-Infraspinatus and teres minor.

Internal rotators.-Latissimus dorsi and subscapularis.

Flexor.-Coraco-brachialis.

Extensor.-Teres major.

Deltoid.-This thick fleshy triangular muscle which gives the rounded form to the shoulder arises from the outer third of the clavicle, outer border of the acromion and the lower margin of the spine of the scapula. The fibres converge 
from this extensive origin to a narrow tendinous insertion at the middle of the outer side of the humerus. Though limb muscles usually have their insertion nearer to the

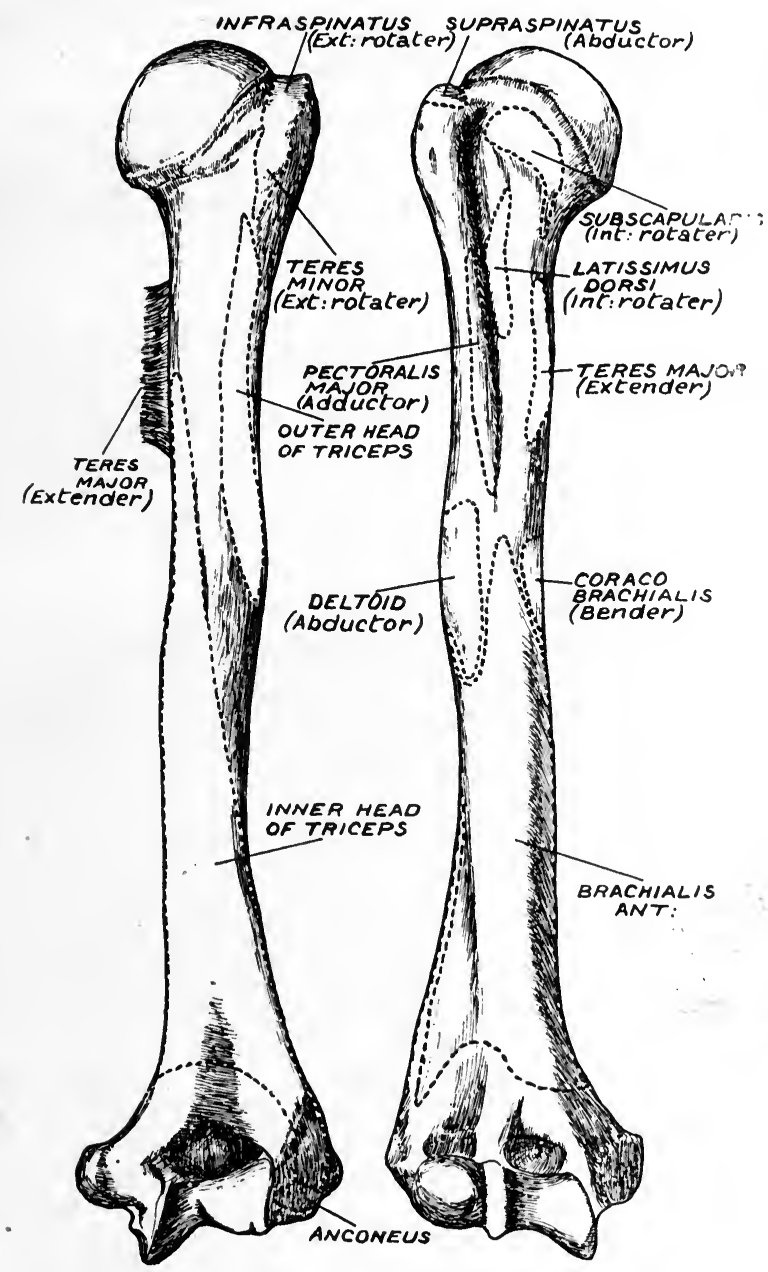

FIG. II.-The insertions of muscles moving the humerus.

centre of motion than the origin, yet in the case of the deltoid and coraco-brachialis the origin is nearer the centre of motion than the insertion. The effect however is the 
same in each, action being produced with a minimum contraction. The insertion of the deltoid into the humerus corresponds to a triangular area about $2 \frac{1}{4}$ inches long with a greatest width of about I inch. The width of its origin is about 7 inches. Thus a muscle whose width of origin is 7 inches operates on an area whose width is only $I$ inch.

Supraspinatus.--This is so called because it arises from the hollow on the back of the scapula above the spine. It becomes adherent to the shoulder capsule, and is inserted into the highest facet of the great tuberosity of the humerus. By its attachment to the capsule of the shoulder joint, this, though lax, is prevented from being impacted between the humerus and glenoid cavity when the arm is raised above the head.

Pectoralis Major.-This arises from the front of theinner half of the clavicle, from half of the front of the sternum and costal cartilages as low as the seventh cartilage, and by a slip from the aponeurosis of the external oblique of the abdomen. The fibres converge towards the upper arm, and the muscle twisting on itself is inserted by a flat tendon about $\mathrm{I}_{\mathbf{4}}^{\mathbf{3}}$ inches wide into the outer or lateral margin of the groove in the upper third of the humerus for the passage of the biceps tendon.

Infraspinatus.-This thick and fleshy muscle arises from the infra-spinous fossa on the back of the scapula and is inserted into the capsule of the joint like the supraspinatus, and also into the middle facet of the great tuberosity of the humerus.

Teres Minor.-This like the teres major is so called from its apparent roundness when first dissected. It arises from the lower or axillary margin of the scapula and is inserted into the lowest of the three facets on the great tuberosity of the humerus, and into the bone immediately below it. It is worth remembering that the strong fibro-muscular attachment of the infraspinatus and teres minor measures about 2 inches in width.

Latissimus Dorsi.-This broad flat muscle arises in the loins, being attached to the spines of the six lower thoracic, 
lumbar, and sacral vertebraæ, also from the back of the crest of the ilium and by fleshy digitations from the dorsal portions of the four lower ribs. It is traced up and out round the side of the trunk, the fibres converging on the dorsum of the spine of the scapula into a thick fasciculus which twisting on itself is finally inserted by a flat riband-like tendon on the bottom and inner edge of the bicipital groove opposite that of the pectoralis major. One is struck by the relatively weak insertion for such an extensive muscle which is less pronounced than the much smaller muscle-the teres major.

Subscapularis.- This is a fleshy, strong, somewhat fanshaped muscle, lining like a cushion the ventral surface of the scapula from which it arises. Its fibres are inserted by a broad thick tendon into the lesser tuberosity of the humerus, and into the neck and shaft of the bone below the tuberosity for about $\mathrm{I}$ inch. This muscle at its insertion measures about $\mathrm{I}_{\frac{3}{4}}$ inches in width.

Coraco-Brachialis.-This narrow but fleshy muscle arises from the apex of the coracoid process and is inserted at the middle of the inner surface of the shaft of the humerus. This muscle is fleshy almost to its insertion, which is equal in length to, but slightly narrower than, the teres major.

Teres Major.-This arises from the dorsal aspect of the lower angle of the scapula and is inserted by a flat tendon into the inner or medial margin of the bicipital groove close to that of the latissimus dorsi. If we examine a humerus specially preppared to show the insertion of muscles we find that the tendon of the teres major lies, at its insertion, not merely medial but distinctly dorsal to that of the latissimus dorsi. The insertion of the teres major is seen when the bone is viewed from the dorsal aspect. This has an important bearing on the function of the teres major. Though this muscle is usually described as an internal rotator, it is important to remember that we can flex or extend the humerus in the position of external or internal rotation at the shoulder joint. 


\section{(2) Muscles which act on the Scapula}

These are :

Trapezius.-This rotates the scapula upwards, backwards towards the spine, and outwards (elevation) from the chest wall, rotating also the clavicle upwards and backwards. It is assisted by a direct elevator acting at the superior angle, the levator scapulæ, which arises from the transverse processes of the upper four cervical vertebræ, and is inserted into the vertebral border of the scapula from the superior angle of the spine on the dorsal aspect.

Serratus Magnus or Anterior.-Its action is to draw the scapula inwards to the chest wall, downwards, and forwards. It is the antagonist of the trapezius, and is assisted by the subclavius, which passing from the first rib near its cartilage to the under surface of the middle third of the clavicle draws the clavicle downwards and forwards.

Rhomboids. - Draw the scapula backwards towards the spine and somewhat upwards.

Pectoralis Minor.-The antagonist of the rhomboids draws the scapula forwards and somewhat downwards.

Trapezius.-This muscle receives its name owing to its lozenge form and to older anatomists was known as the cucularis from its resemblance to the monk's cowl. This fleshy muscle arises above from the skull, being attached to the external protuberance of the occipital bone and the adjacent superior curved line; from the ligamentum nuchæ which extends from the protuberance to the seventh cervical spine, and from the spines of all the thoracic vertebræ. The upper fibres pass down and out to be inserted into the outer third of the clavicle, the intermediate fibres pass out to the acromion and upper border of the spine of the scapula, and the lower fibres pass up and out and form a flat tendon which is inserted into the root of the spine of the scapula.

Serratus Magnus or Anterior.-This fleshy muscle lies upon the side of the chest extending between the ribs and the scapula. It arises by a series of fleshy tongues or 
digitations from the lateral aspect of the upper eight ribs. Its insertion is into the whole length of the vertebral border of the scapula on its ventral or anterior aspect.

Rhomboids (major and minor).-The origin is from the lower part of the ligamentum nuchæ and the spines and supraspinous ligaments of seventh cervical and upper five thoracic vertebræ. The insertion is into the vertebral border of the scapula on its dorsal aspect extending from the spine of the scapula, to the inferior angle.

Pectoralis Minor.-This triangular muscle lies beneath the pectoralis major. It arises by three digitations from the third, fourth, and fifth ribs near their cartilages, and is inserted by a flat tendon into the coracoid process of the scapula.

\section{Elevation of the Upper Limb}

When we speak of paralysis of the shoulder or scapular region, we invariably refer to the inability either to raise the arm to a right angle, or the arm and hand above the head. The exact mechanism by which we raise the hand above the head has been the subject of discussion for many years. In the first place it may be observed that the upper extremity is connected with the trunk only at one relatively small point, the sterno-clavicular articulation. At the outer end of the clavicle, where it is related to the acromion, strengthening the acromio-clavicular capsule, we have the firm coraco-clavicular ligaments, the conoid and trapezoid. Here there is little movement of the scapula on the clavicle, and Bell regarded this as almost a fixed point. The deltoid assisted by the supraspinatus abducts the arm to a right angle. Farther movement of the humerus upwards is prevented by the acromion projecting over it. This farther movement is not performed at the shoulder or gleno-humeral joint, but is rendered possible by a rotatory movement of the scapula and the clavicle on the trunk.

The principal motion takes place at the sterno-clavicular articulation, and the clavicle has been well described as a shaft or axis on which the scapula rotates together with 
the humerus, which is fixed to it by the deltoid and supraspinatus. As the scapula does not appear to rotate through an angle of 90 degrees in this movement, Cathcart held the view that the final part of the movement in this second arc took place in the shoulder joint. The principal motor machine in this second movement is the trapezius which has been described as a continuation of the deltoid, both being attached to the outer third of clavicle, acromion, and spine of the scapula. The trapezius in this motion is assisted by the levator scapulæ and the rhomboids. By means of the serratus magnus passing from the ribs to the ventral surface of the vertebral border of the scapula, the latter maintains its relation with the chest wall. This is a position necessary for scapula rotation and for depression of the limb after elevation-in this latter action the serratus is assisted by the subclavius. Where this is interfered with as in serratus paralysis the scapula is elevated from the chest wall and raised upwards, giving rise to the characteristic winging of the scapula; and then elevation of the hand above the head is not possible (see figs. I2-I7). In paralysis of the deltoid, trapezius, or serratus, the reason why the patient consults us is the inability to raise the hand from the side above the head.

Positions of Anatomical Rest for Loss of Muscular Power in THE SHOUlder Region

Trapezius.-In a case of loss of muscular power in the trapezius muscle, which is not so common as that of the deltoid, the arm should be abducted to an angle of not less than 145 degrees. This can be easily effected by means of an upper limb abduction splint the stem of which is tilted up at the axilla. While the splint is being made, to effect this the sleeve can be immediately pinned to a pillow, and another pillow placed in the axilla with the patient in bed. I prefer the metal splint to any plaster appliances, a great advantage being that it can be removed in a few seconds for the purpose of muscle re-education. 


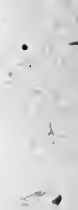

I 

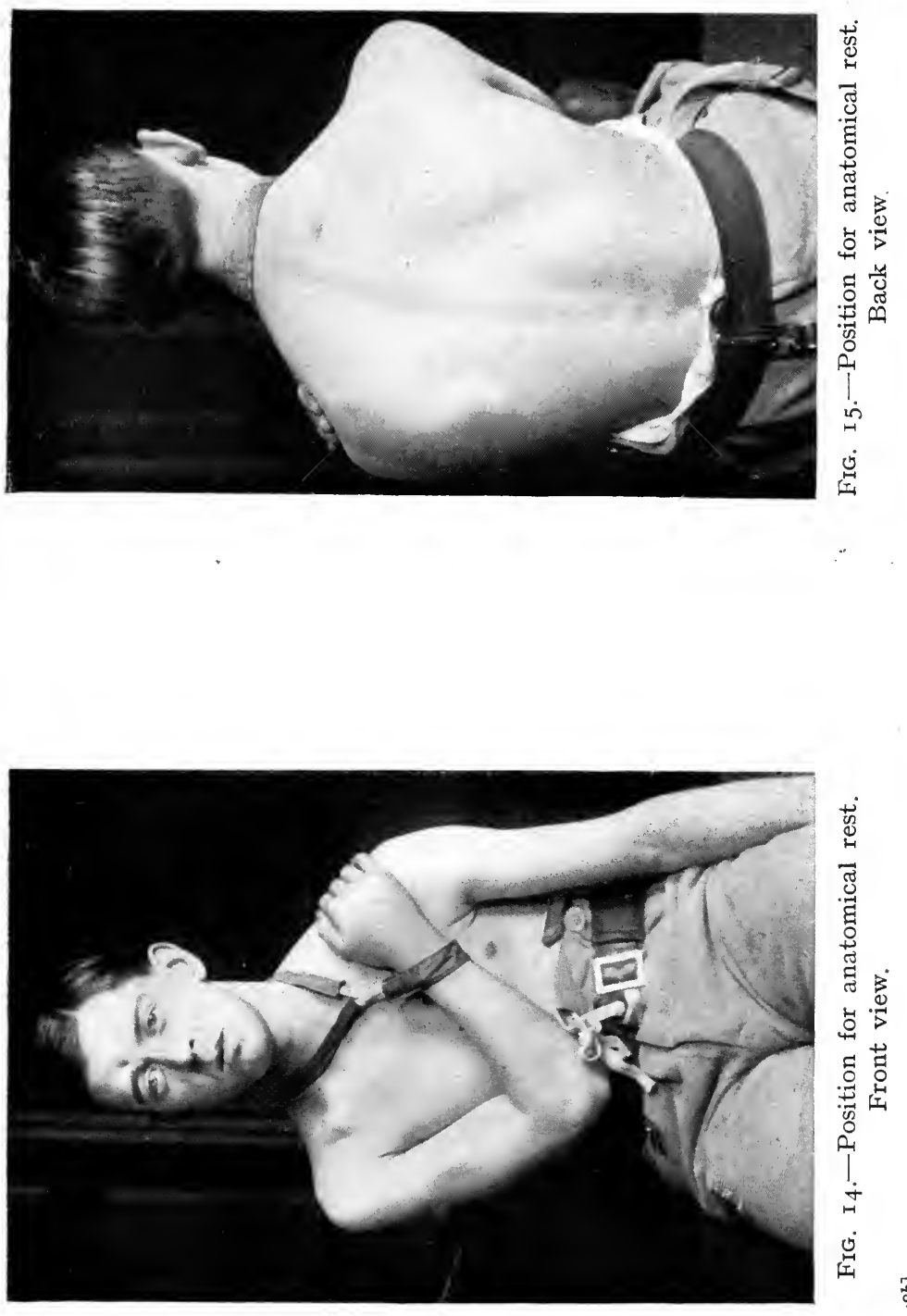
1

$\because=\cdots x_{x}$ 


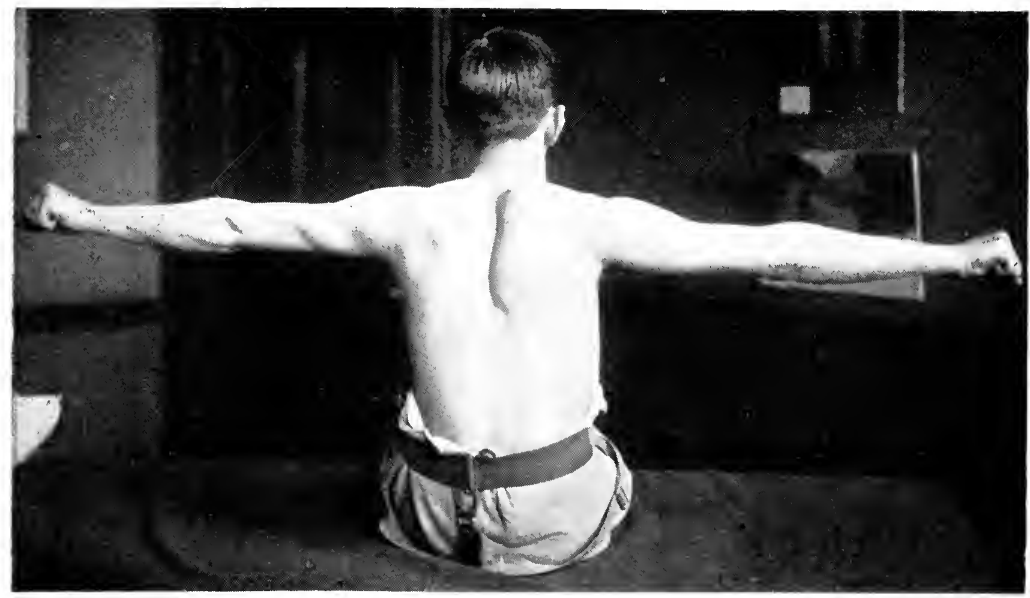

FIG. I6. - The inability when erect to elevate the upper limbs beyond the right angle.

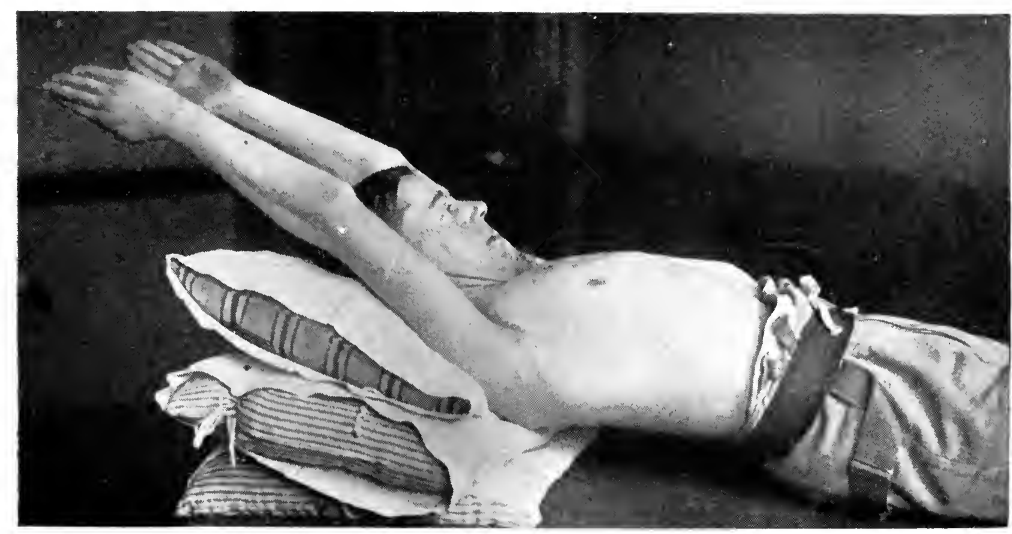

FIG. 17.-Method of re-education. 
Deltoid.-Here a similar procedure is adopted, the stem of the abduction splint being placed at a right angle-and the scapula rotated backwards. In this way the contraction of the antagonist pectoralis is best prevented. The following is the model of the splint (fig. I8) first introduced by me at the Australian Medical Congress, October I908, and exhibited at Bologna, Italy, in roog.

The basis is a stem of malleable iron $\frac{1}{2}$ inch wide and $\frac{1}{8}$ inch thick. This runs up the mid-axillary line from about $x$ inch above the costal edge to the axilla and follows the required contour of the under surface of the arm and forearm to the

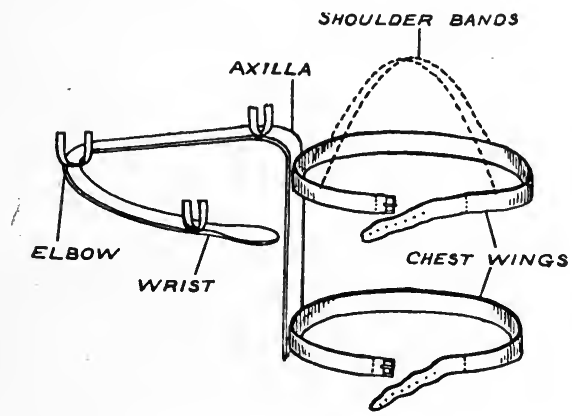

FIG. I8.-The upper limb abduction splint.

wrist, being bent to the requisite angle at the axilla and elbow. At the latter, flexion almost to a right angle will be found most suitable for the patient. A piece of aluminium or tin shaped to the hand is attached at the wrist termination so as to allow the mid prone and supine position, and the fingers rest on a pad of wool. Wings $\frac{1}{2}$ inch by $\frac{1}{16}$ inch are attached at the wrist, elbow, and upper arm, and two wings pass round the chest being attached to the stem, one at the costal extremity and the other below the apex of the axilla. The ends of these chest wings are connected anteriorly by strap and buckle. The limb is lightly bandaged on, and pieces of webbing crosswise over each shoulder from the back to the front are sufficient to hold the splint well into the armpit.

Serratus. - The hand of the affected side is placed on the 
opposite shoulder with the elbow at the side and maintained in this position by means of a bandage sling round the neck (see figs. I4 and I5). In this position all winging of the scapula disappears, the scapula is brought downwards, forwards, and inwards to the chest wall, while the opponents of the serratus are relaxed and elongated. There is little doubt that in cases of loss of power about the shoulder rest in bed at the time of injury or after nerve operation should be insisted on as much as possible. In an early infantile paralysis case, this should be the rule until recovery.

Method of Re-education For Muscular Weakness in THE Shoulder Region

A paralysed limb should at all times be handled with the greatest gentleness. The muscular engine will not respond to force, but will respond to gentle coaxing. When taken off the splint with the patient lying down the limb should occupy at first the position it had when supported by the splint. The arm with a paralysed deltoid should not be allowed to drop when the splint is removed. We should be as careful to avoid unnecessary movement as in the case of a fracture of the humerus. Hence also the importance of a firm bed, so that movements can be carried out without unduly disturbing the patient. At first it is best to place a sheet of powdered cardboard under the limb to obviate all resistances to movement. In re-education to enable the patient to raise the hand from the side to above the head three separate arcs of a circle must be carefully borne in mind. These patients invariably have the volitional test applied when standing or sitting erect. But of one thing we can be certain, if the patient cannot elevate the arm when lying flat on the back either on a table or firm bed, he will not be able to accomplish this when erect. It is impossible to perform Ioo units of work if the muscles fail at Io. We give the minimum test for recovery by commencing work with the patient lying flat and with a folded sheet under the head. We commence at "zero" (A B, fig. I9), the patient 
being gradually elevated by means of pillows placed under the head and shoulders along the planes $\mathrm{E} \mathrm{B,} \mathrm{C} \mathrm{B,} \mathrm{and} \mathrm{H} \mathrm{B}$, till the sitting erect plane D B is reached. Apart from this

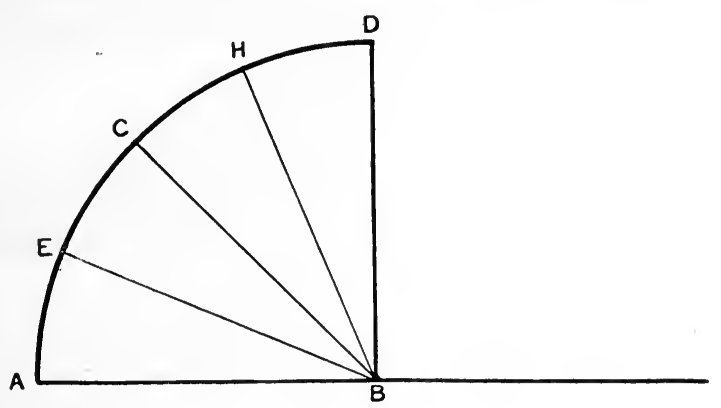

FIG. I9.

arc, which refers to the position of the body relative to the limb, we have for consideration the fact that deltoid abduction and scapula rotation also represent two distinct arcs.

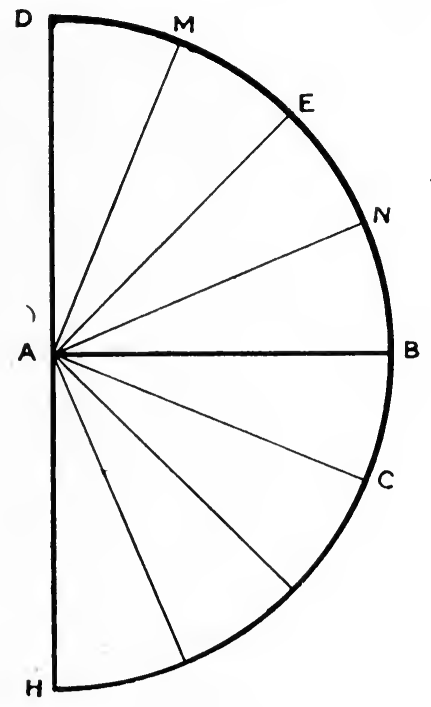

FIG. 20.

In order to re-educate weak muscles of the shoulder region these two separate arcs of deltoid abduction, $\mathrm{H} \mathrm{B}$ (fig. 20) and of scapula rotation B D (fig. 20), must be 
considered in every case. Work should begin in the absolutely horizontal plane A B' (fig. I9), full movement obtained in that, and then gradually increased to the full vertical plane D B (fig. I9) in the manner described. Full movement in both arcs of deltoid abduction and of scapula rotation must be obtained in any one of the body planes E B, C B, H B (fig. I9) before proceeding to the next highest-this next one is then reached as stated by gradual elevation of the patient.

In fig. 20 the line A B represents the arm held abducted to a right angle. It represents the maximum work, or roo units, for the deltoid in whatever plane of the body we are working. But the completion of the arc $\mathrm{H}$ B does not necessarily mean that we can enter the arc B D (scapula rotation). In commencing work in the $\operatorname{arc} \mathrm{B} \mathrm{D}$ at $\mathrm{A} B$ we are asking the muscles that produce rotation of the scapula to perform their work at the maximum disadvantage. With the arm hanging at the side, represented by the line A $\mathrm{H}$, we have the humerus in the position where to raise it the deltoid is asked to do a maximum of work. But the weakened deltoid is incapable of doing that work, and in that position is at its maximum disadvantage. Furthermore, it is in that position overstretched by a dependent limb to its greatest extent. It cannot pull right up the hill. We place the limb at a right angle, keep it supported there, and are certain we can lower it and go from $\mathrm{C}$ to $\mathrm{B}$ before we attempt $\mathrm{H}$ to $\mathrm{B}$. In this way we have a certain means of testing recovery and of regulating the amount of muscular work. We have not only to coax our muscle to functionize with the limb in the easiest position, but similarly with the position of the limb relative to the rest of the body.

Deltoid Re-education.-With the patient lying on his back on a firm bed or table and with a slight support under the head we may find that he lifts the abducted arm readily off the cardboard, or if it be passively raised he may be able to maintain it so (fig. 2I). Our object is to recover the power of abduction from the side to a right angle. If 
we lower the arm from the right angle abducted position to the side, the patient is unable to abduct to the right angle, but if we lower it down to A C (fig. 20), supporting the patient's elbow on the palm of our hand, it may be raised to the right angle readily. We gradually lower the supported elbow, beginning each day at our original minimum till finally we reach the position with the arm at the side. There will be recovery in this posture when the patient can abduct to the right angle at once with the arm at the side. Until this deltoid arc is completed the patient is unable voluntarily

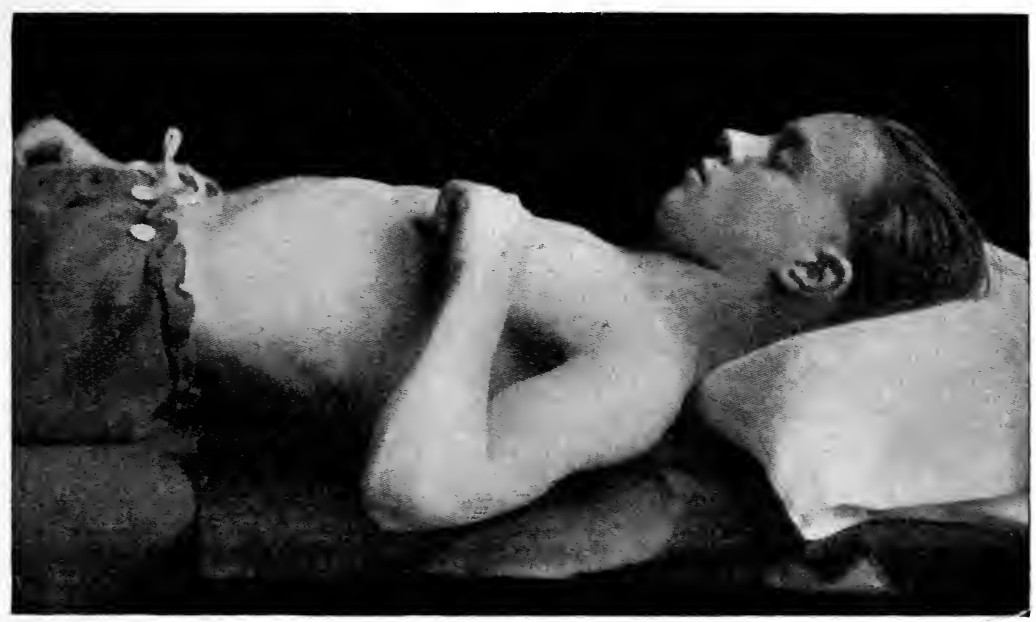

FIG. 21.-Method of re-educating the deltoid.

to enter the second or scapula arc. In many cases I have seen, this has been unattainable, not so much on account of the weakened deltoid as of a contracted pectoralis and occasionally of the latissimus dorsi. The position of the forearm and hand in testing for deltoid recovery, or in commencing re-education, is important. With outward rotation at the shoulder, flexion at the elbow to a right angle, and supination of the hand, deltoid action is aided (fig. 22). It is less easy with the elbow extended and the hand supinated. It is difficult with the hand over-pronated, the elbow flexed, and inward rotation at the shoulder. In an old neglected 
case of infantile paralysis, with associated weakness of flexors and extensors giving rise to " flail elbow," it is best at first to fix the elbow by means of adhesive plaster in the

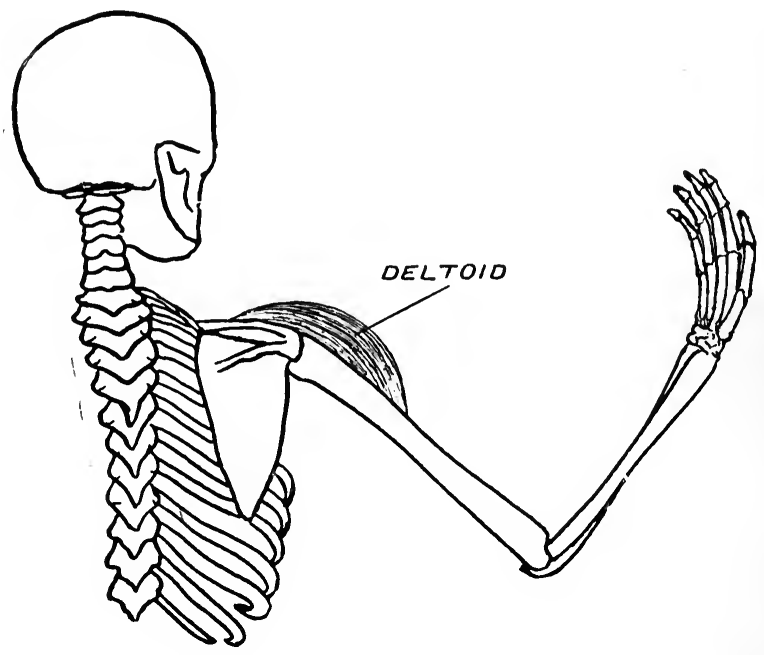

FIG. 22.-Illustrating method of educating the deltoid.

flexed position, so as to improve our leverage for abduction movement.

Scapula Re-education.--The limb having been passively brought into this arc we work slowly from A M (fig. 20), not from $\mathrm{A} \mathrm{E}$ or $\mathrm{A} \mathrm{N}$, along the arc $\mathrm{M} \mathrm{D}$, this having been found the easiesi position from which to begin movement. From there we gradually increase the work by moving downwards towards the plane A B. The Ioo units of work or maximum effort are completed when the patient can move the limb along the arc B D. We cannot work from A E to A D till we are assured of $\mathrm{A} M$ to $A \mathrm{D}$, nor similarly from $\mathrm{A} \mathrm{N}$ till we are assured of A E. All the time we support the patient's extended elbow with our hand. It may not be necessary to wait for the completion of the deltoid arc before commencing with the scapula arc. The fact that voluntary movement can take place in the latter enables one to give a hopeful opinion as to the probability of recovery of movement in 
the deltoid arc in an old infantile paralysis case. Reeducation in the two arcs may go on together, but we know that until deltoid abduction to a right angle is complete, we cannot lift our arm from the side above the head in one continuous movement. Re-education movements should be carried out for twenty minutes about four times a day. These procedures are similarly repeated with the patient slightly raised by means of an ordinary pillow under the head, and in this manner he is gradually elevated to the orthograde or erect posture. The Ioo units of work or normal have been achieved when the patient is able sitting erect to abduct the arm from the side not only to a right angle, but to elevate it also above the hea'd.

As illustrating the difficulties we may have in recommencing function and the various means adopted in seeking for muscular advantages, the case of an adult with infantile paralysis of about two years' standing may be cited. Both upper limbs had been affected, and throughout this period he was not able to raise either arm to a right angle. After four weeks' treatment on the lines of the preceding principles he was able when lying down and elevated on two pillows to raise both hands above the head. When seen the patient was placed immediately in a double upper limb abduction splint. The right upper extremity was the worse of the two, and to this I shall refer. He was tested lying flat on a firm table with a single pillow under the head and a sheet of powdered cardboard under the limb, to obviate friction. With the hand at the side, beyond a jerk of 2 to 3 inches out he was at first quite unable to abduct the limb. I then placed the arm with the elbow extended in the second arc, i.e. elevated it to an angle of about I 40 degrees, A E (fig. 20), and found the patient elevated it to I80 degrees, A D (fig. 20). It did not fall to the side. With coaxing I found he could elevate it from Ioo to I8o degrees.

As a result of this the opinion of possible return of abduction power, of deltoid action, became slightly favourable. In a week when lying flat with the arm resting on a pillow 
(still using the cardboard) and with the elbow extended, he was able to jerk the limb, and in this way to abduct it from the side up to the right angle from which, using the pillow as a support, he elevated it above his head with ease. One movement only of this nature was at first a great effort for the patient, but it represented the best means of antagonizing the more powerful pectoral. It was then found that if we took the pillow away from the side so as to increase the effort, he was unable to elevate the limb. If however we used first a smaller pillow and then a folded sheet he finally elevated the arm from the side without pillow support. Gradually in this position he abducted the limb to the right angle with less jerk and more true volitional effort. I found also that though there was difficulty in elevating with the elbow extended, yet if I flexed the elbow (the brachialis though functional was weak), shortening the length to be elevated, the patient could raise the arm from the side above the head-at first with some effort, and later with comparative ease. Within three weeks an arm condemned after nearly two years as hopeless for recovery could be raised by the patient's volition from the side above the head in the lying-down posture; and similarly a week later when the patient's head and shoulders were elevated by two pillows. As the limb was raised from the beginning more easily with the elbow flexed than with the elbow extended the line of treatment was obviously to regard the flexed elbow as the zero- the extended elbow position being gradually reached by means of a light splint or adhesive plaster.

The first cases of infantile paralysis with loss of abduction power I ever treated by this method were a series of ten seen in Australia during an epidemic of this disease in I908.* They were all immobilized for from ten to fourteen weeks, and of these nine recovered. They were not cases which could have recovered spontaneously, as the improvement was very gradual. The case that did

* "Treatment of Infantile Paralysis of the Upper Limb." Intercol. Med. Journ. of Aust., May I9og. 
badly was that of a child with four limbs affected and the muscles of the back and neck, in addition to having marked meningeal symptoms. Within an hour after being seen all cases were immobilized by means of the abduction upper limb splint, and when possible, recumbency obtained by means of a double Thomas hip splint. Personally at the outset I prefer complete recumbency with even one limb affected, using, not owing to any particular virtue for this condition, but for its convenience, the double Thomas hip splint. The limb was well surrounded with wool and rested completely for a week. At the end of that time the limb was taken off the splint for purposes of re-education, being placed on a pillow in the position occupied when in the splint. Neither massage nor electrical treatment was allowed in these cases until recovery in the erect position was reached. Massage does not initiate muscular function; it does not permit rest of an inflamed cell. These results were shortly afterwards confirmed by E. Mayer of Cologne, who in one of his cases with affection of both arms, leg, and back, obtained complete recovery in two months except for a slight weakness of the tibialis anticus.*

\section{Pectoral Contraction: Stiff Shoulder}

The importance of pectoral contraction (occasionally also that of the latissimus dorsi) in preventing arm elevation cannot be too strongly insisted on. Not only should the arm be abducted, but rotated backwards, so as to obviate all chance of contraction occurring. Frequently one sees an abduction splint applied in which the hand is brought forward on a level with or beyond the patient's mouth, thus favouring pectoral contraction. In re-education the greatest antagonistic force is felt as we approach the right angle A B (fig. 20), and until this is overcome we may move readily from $\mathrm{H}$ to $\mathrm{C}$ and be unable to move from C to B. The possibility of a "stiff shoulder"

* International Clinics, vol, i, I912. 
following any injury or inflammation of the shoulder region should always be borne in mind and means taken to prevent it. I have seen it occur after rheumatic inflammation of the shoulder, fractures of the upper end of the humerus, falls on the shoulder, and after bullet wounds of the neck region and of the shoulder joint without much obvious injury to muscle. By a stiff shoulder we do

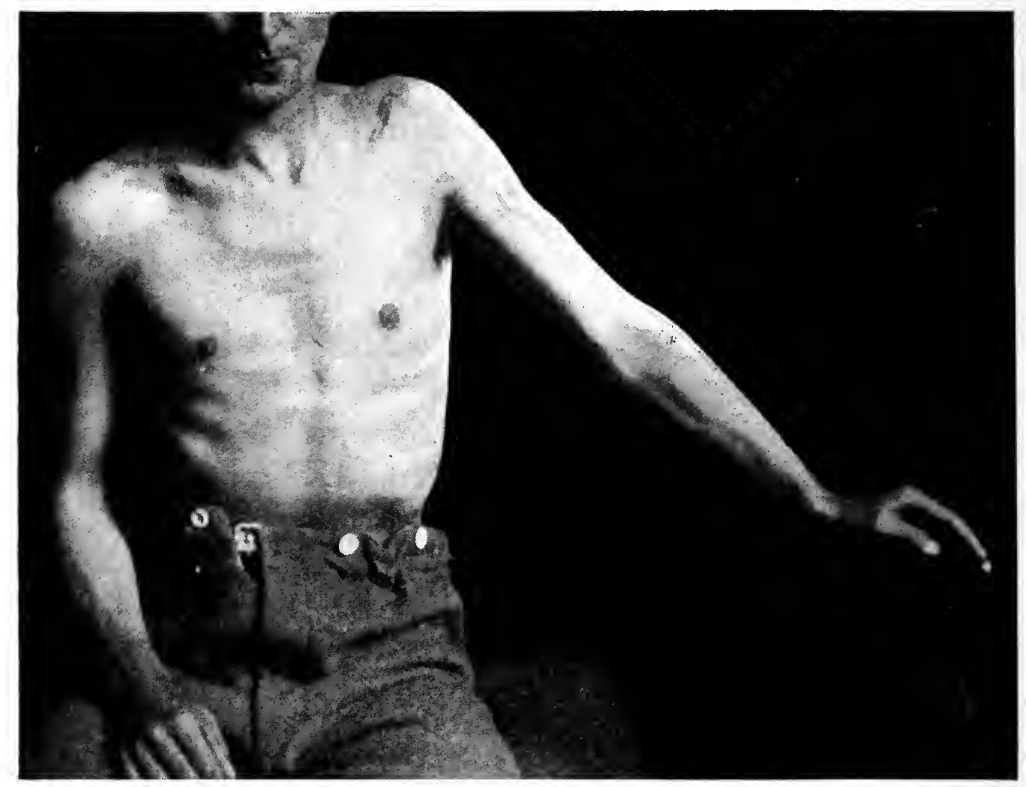

FIG. 23.- Stiff shoulder.-The patient when erect is unable to abduct the arm 45 degrees from the side.

not necessarily mean "adhesions" in the joint binding the head of the humerus to the glenoid cavity. In many cases of stiff shoulder the head of the humerus can be rolled freely on the glenoid. The two factors to be borne in mind are adductor contraction, which is specially favoured by gravity, and weakness (paresis) of the muscles, which elevate the limb. The former responds to continued pressure, though in an old case it may be necessary to lengthen the pectoral tendon, and the paresis will yield to treatment on the 


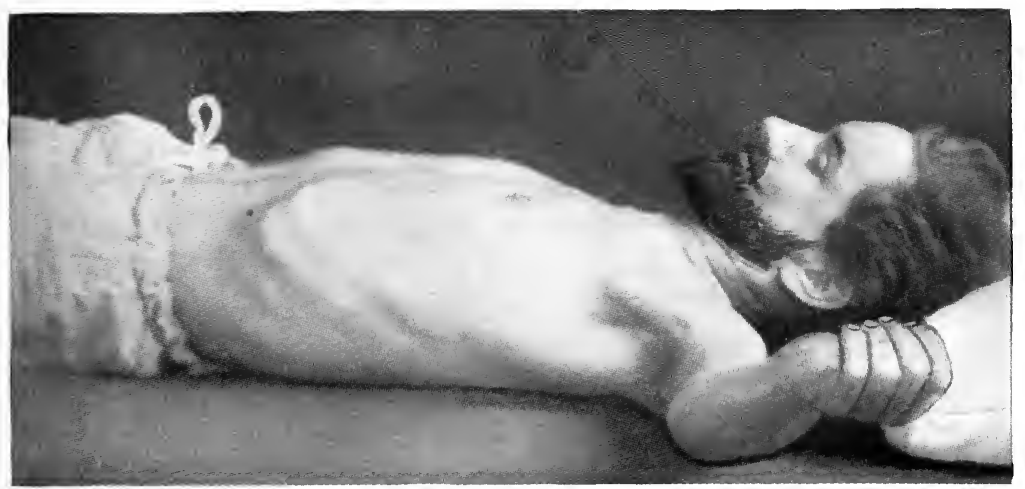

FIG. 24.-When lying flat on a firm table the same patient abducts the arm readily to a right angle.

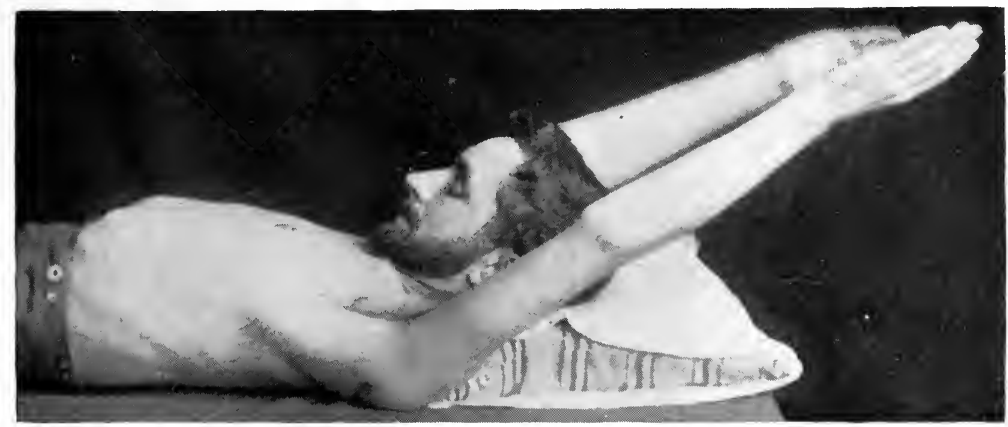

FIG. 25.-When lying flat the same patient can partly elevate the left upper limb above the head, i.e. can enter the second or scapula rotation arc. 


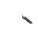

$\because 2$ 
previously indicated lines of gradual re-education. These cases are numerous. They have usually had massage of muscles assiduously for many months, and the "adhesions of the joints" have at various times been broken down under an anæsthetic without alleviation of the condition. Muscular function as a factor in restoration has been regarded as unworthy of consideration.

My attention was first drawn to these conditions by the case of a woman who, thirteen months before being seen, had fallen on her right shoulder. Pain, swelling, and stiffness followed, for which massage, electricity, and forced movements "to break down the adhesions" were used. On examination, in spite of the fact that the head of the humerus could be rotated freely, there was inability to abduct the arm, when sitting, more than two inches from the side. When lying down however, with a small pad under the head, she was able to her astonishment to abduct the arm to a right angle, and though with difficulty, owing to some contraction of the pectoralis, to raise it above her head. She was thus not only able when lying down to complete the arc H B (fig. 20), but could practically complete the second arc B D. The limb was placed on the abduction upper limb splint to stretch the adductor.

When the splint was taken off a week later the patient was still unable to abduct the arm when sitting up, but on lying down she raised it above her head with ease. In two days she was able to raise it, not only when lying flat, but also when the head was elevated on a pillow. Within a fortnight she was able to raise the hand above the head when sitting up, having during that time been gradually raised by pillows to the erect position, the movements each day being commenced in the lying-down or "zero" position. A week later she was able to raise the arm immediately on sitting up. Till then the splint was worn. Such a rapid recovery is however rare. Treatment usually extends over a much longer period. The patient, in this as in all cases of muscular affection, must be encouraged 
to take an intelligent interest in his or her own condition; and in this way any superadded hysterical or functional element in the case may be successfully eliminated. With a pillow on the table or floor for twenty minutes four times a day the attempt at previous maximum effort should be repeated. The volitional effort of the patient can be greatly aided by gentle but firm pressure on the contracted pectoralis major, and the patient should also make traction with his sound hand on the affected limb. 


\section{THE BICEPS BRACHII AND BRACHIALIS: MUSCULO-CUTANEOUS PARALYSIS}

THE musculo-cutaneous nerve, which derives its origin from the fifth and sixth cervical, supplies two muscles, the brachialis and biceps brachii. The coraco-brachialis, though pierced by the nerve, receives its supply from the seventh cervical. Occasionally, it is stated, an additional fine branch can be traced from the musculo-spiral to the brachialis muscle. This is also derived from the fifth and sixth cervical.

Brachialis. - In the marsupial this is a comparatively small muscle. In man it is strong and fleshy. It arises by an extensive and largely muscular origin from the lower half to two-thirds of the outer and inner surfaces of the front of the humerus, and crossing the elbow joint has its insertion into the front of the coronoid process of the ulna (fig. II, p. 53). In the student's mind the power of this muscle is over-shadowed by that of the biceps. The strong grip of origin this muscle has is well shown in the magnificent dissection of the humerus demonstrating the attachment of the different muscles prepared by William Pearson at the London College of Surgeons.

The brachialis throughout its extent is essentially a fleshy muscle compared with the biceps. The muscular character is continued almost to the insertion, and in a well-dissected specimen of the bend of the elbow, the main biceps tendon appears as an insignificant structure compared with the broad muscular brachialis in the background. This muscle is the bender of the elbow joint, and its antagonist is the 
triceps (extender). The brachialis instead of flexing the forearm on the arm, in which case the arm is the fixed point, may produce the reverse action and flex the arm on the forearm, in which case the forearm is the fixed point. This is seen in elevation of the body on the horizontal bar (fig. 26),

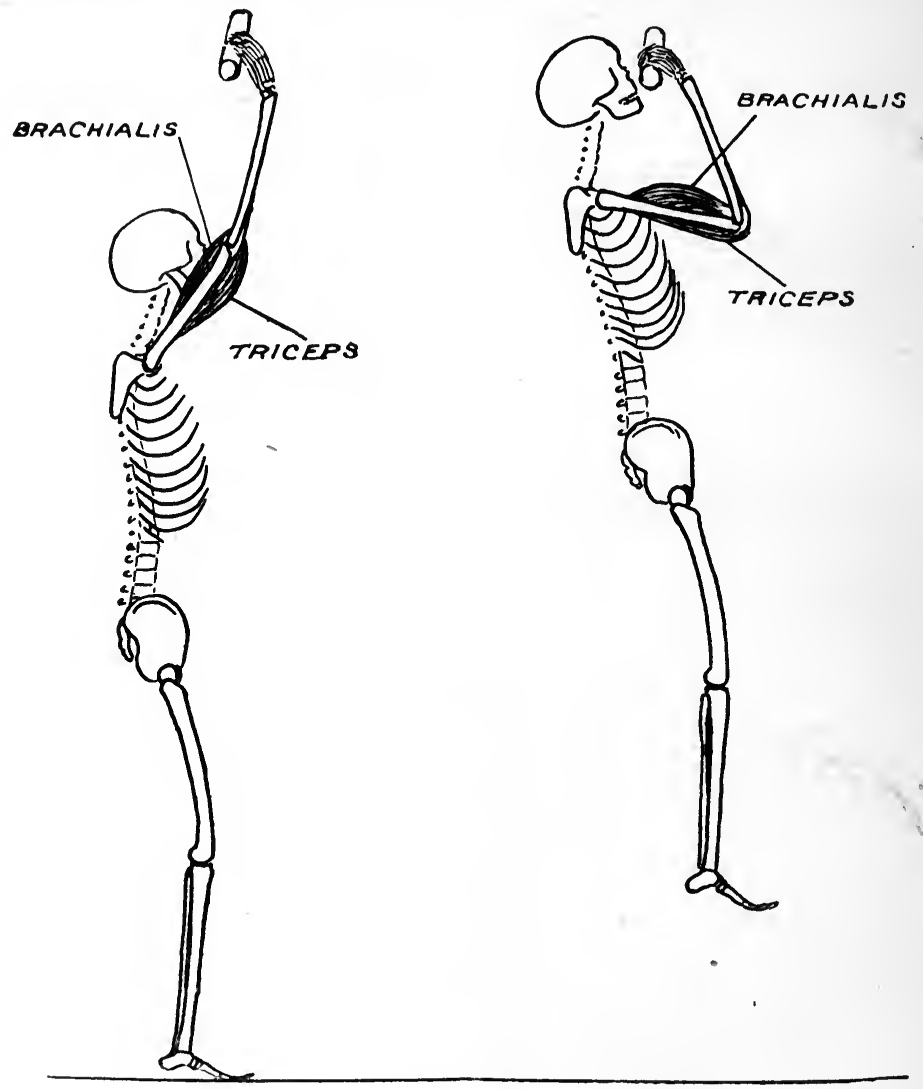

FIG. 26.-Illustrating "reverse action" by the brachialis muscle.

the origin of the brachialis being the insertion, and the insertion the origin. When the athlete grasps the bar with the hands to raise himself he does so with the hand either in the position of over-supination or over-pronation. If the former, the biceps is contracted; if the latter, it is relaxed and elongated. Elevation of the body really consists of 
flexion of the arm on the fixed forearm-the amount of elevation depending on the degree of flexion. This action is performed by the brachialis, and the triceps its opponent is relaxed and elongated.

The brachio-radialis or supinator longus is regarded as an accessory flexor of the elbow joint. Both pronating and supinating powers have also been ascribed to this muscle, the power therefore of contracting and relaxing at one and the same time. In man this muscle would appear to be struggling to retain its position in the forearm. If its insertion be examined closely in a well-dissected specimen the tendon is found not merely to be inserted into the lateral edge of the lower end of the radius, but on to the anterior surface as well, it comes well round to the front at its insertion. This in man would correspond to the outer more tendinous portion of the insertion as seen in the wombat. The centre of motion for muscles inserted into the radius is radio-ulnar, not humero-ulnar, and I regard this muscle as an accessory supinator especially to the biceps.

Its greatest development is reached in the powerful fore limb of the wombat (fig. Io, facing p.50), and its origin reaches as far as the spine of the scapula. Here it acts as a supinator, being inserted by a broad attachment into the outer margin and dorsum of the lower end of the radius. In the koala it has an extensive origin from the humerus, and crossing dorsal to the radio-carpal articulation is inserted by a welldefined tendon into the outer side of the carpus. In the poorly developed fore limb of the kangaroo it is largely tendinous, and closely related below to the lower portion of the radius where it terminates.

Biceps Brachii.-This arises in man by two tendinous heads-a short head from the coracoid process, and a long head which arising from the upper margin of the glenoid cavity passes through the shoulder joint. The tendon of the long head is narrow and rounded, while that of the short head is wide. At its origin it is in close relation with the coraco-brachialis, and its under surface of attachment is distinctly muscular. The belly of the short head is nearly 


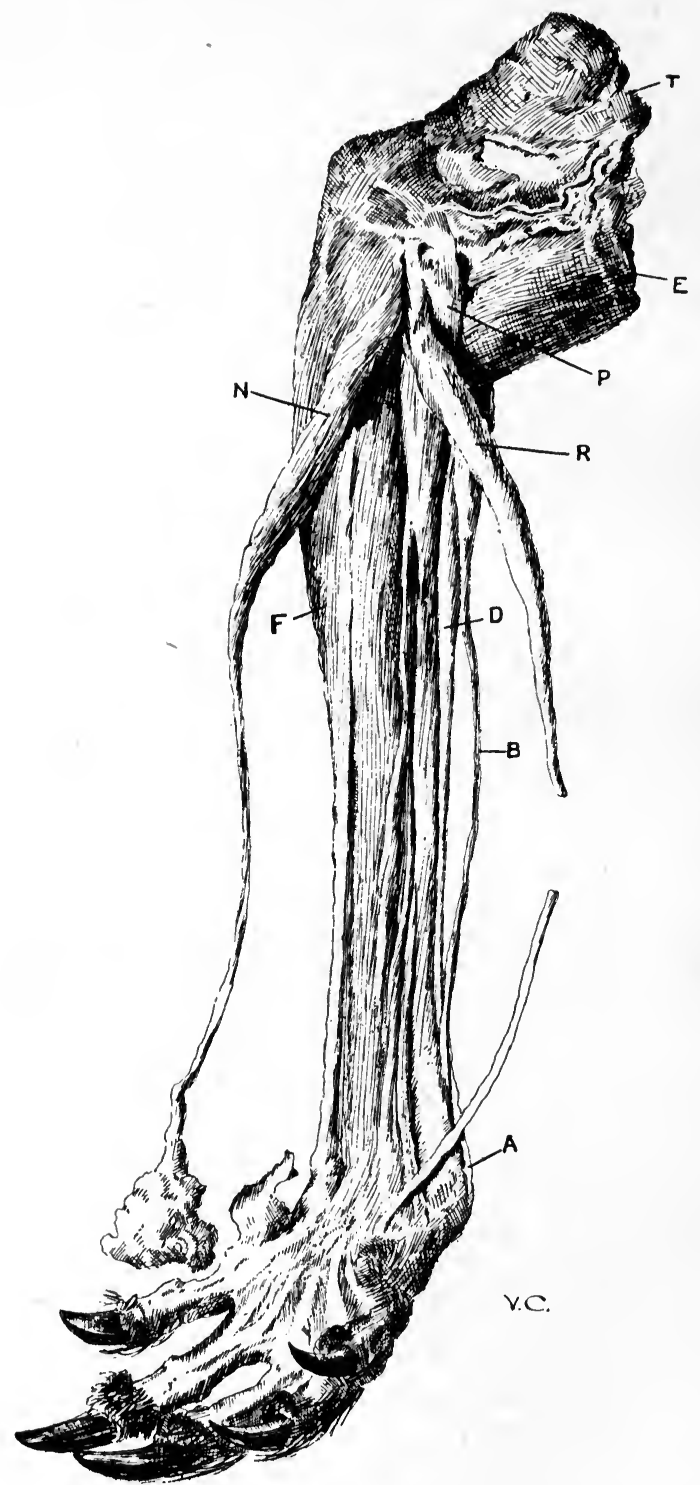

FIG. 27.-Dissection to show front of forearm and hand. Kangaroo.

T, Triceps. E, Biceps. P, Pronator teres. N, Palmaris longus. R, Flexor carpi radialis.

F, Flexor carpi ulnaris. B, Brachio-radialis. D, Wrist extensors. A, Ext: carpi radialis. 
twice as extensive as that of the long head. The two join about the junction of the middle and lower third of the arm. Just above the elbow the fibres terminate in a somewhat flattened tendon which is inserted into the dorsal part of the tuberosity of the radius. In short, the biceps brachii is inserted into the sides of a rotating cylinder.

At the junction of the muscular belly and the tendon of insertion we have arising a membranous band-the so-called bicipital fascia-about $\frac{1}{2}$ inch broad and running on the anterior surface of the tendon from its outer to inner side. It is attached to the deep fascia of the upper third of the forearm. The bicipital fascia does not receive the insertion of any muscular fibres, and the direction of the biceps tendon from the inner to the outer margin is wholly towards the tuberosity. This fascia would appear to act as a band somewhat similar to the annular ligaments or folds, and to prevent undue prominence with liability to injury of a well-defined tendon. It is essential that tendons should be firmly held down to the bone, for if they were allowed to rise during the action of the muscle, the effects of muscular contraction would be greatly interfered with. The long head of the biceps might almost be regarded as a ligament of the shoulder, serving to avoid forcible impaction of the head of the humerus against the coraco-acromial arch during abduction of the arm. Its division is carefully avoided by surgeons during shoulder joint excision.

\section{ACtion OF THE BICEPS}

The biceps is usually regarded as the great flexor of the elbow joint, and as a supinator of the forearm. It is a supinator of the forearm, and so of the hand, but does not flex the elbow-that function being performed by the brachialis. In the first place we would observe that the athlete who displays his biceps during elbow flexion does so with the hand in a position of over-supination. If we over-pronate the forearm, the biceps as a supinator must be in a physiological state of relaxation and elongation. 
Yet with the hand over-pronated we readily flex the elbow. This action is due to the brachialis only. If when half way through the movement of flexion we over-supinate the forearm the biceps is immediately seen to contract and harden (shorten). If we flex still further when over-supinated, and then over-pronate, the biceps is seen to relax and elongate. Further, if the biceps flexed the forearm, when we extended the forearm it would be in a state of relaxation and elongation, yet in this position we can see its contraction on supinating. Thus the biceps acts as a supinator
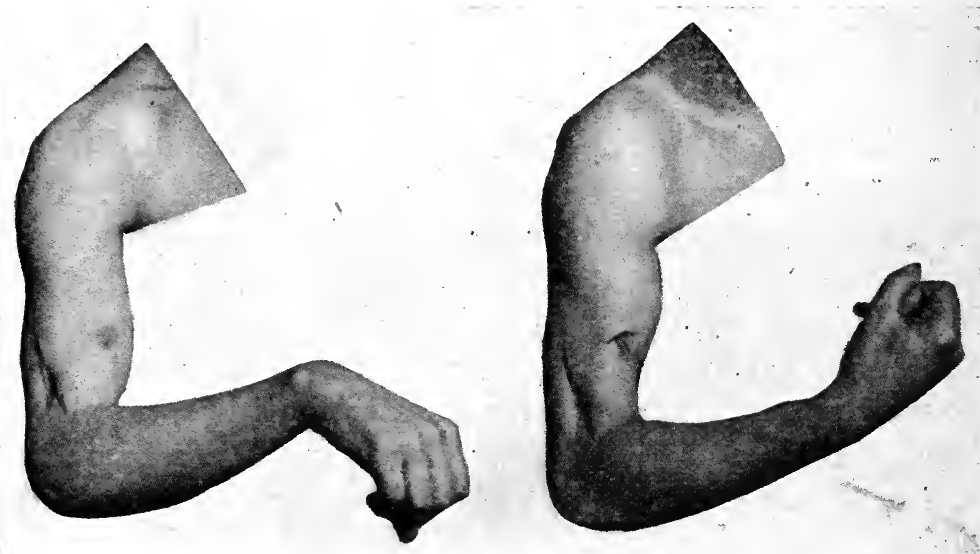

FIG. 28.-Biceps brachii and brachialis. Biceps in pronation and supination of forearm.

in both the positions of flexion and extension of the elbow joint. For its action flexion is most favourable. The moment of its force is greater then than in the extended position. These facts I was able to verify in the case of a soldier with a shell wound of the biceps and adherent skin giving rise to a well-defined scar (fig. 28.) When the biceps was in a state of relaxation and elongation the scar was level with the surface, but when the muscle contracted and shortened it gave rise to a well-defined pit admitting the tip of the little finger. Both the pronator teres and supinator (brevis) cross the elbow joint, the former on the 
ventral aspect and the supinator more dorsally. As both these muscles are inserted into the radius the centre of motion for these muscles is not humero-ulnar (elbow joint) but radio-ulnar.

It may be stated that muscles inserted into the radius do not cause motion in the humero-ulnar, but in the radioulnar articulation. They are either pronators rotating the radius inwards and the palm of the hand down, or supinators turning the radius outwards and the palm of the hand upwards. Their contraction does not cause the physiological state of relaxation in either the flexors or extensors of the humero-ulnar or elbow joint. Extension of the elbow joint is more favourable for supinator brevis action and flexion for action of the pronator teres, although both these muscles can act like the biceps in the flexed or extended condition of the elbow. The pronator quadratus would appear to act best with a firm ulna, as in the position of elbow extension. Pronation of the forearm in the extended position is effected by the pronator quadratus assisted by the pronator teres, and supination by the supinator brevis assisted by the biceps and brachio-radialis. Pronation of the forearm in the flexed position is effected by the pronator teres with possible slight assistance from the quadratus, and supination by the biceps assisted by the supinator brevis and brachio-radialis. Thus, in flexion the effective engines are the pronator teres and biceps, and the subsidiary ones the quadratus and supinator brevis.

It may be argued that if the muscles of the arm and forearm be removed in a fresh cadaver and the biceps alone be left the elbow may be flexed by traction made on that muscle-unmistakable evidence of its power as a flexor. It is of course necessary to know if the traction were made in the natural direction. Furthermore, we are in this case taking no consideration of the opponents of flexion. Elbow flexion is ordinarily permitted, not only because the brachialis contracts and shortens, but because the triceps relaxes and lengthens. The biceps in the natural condition is allowed to act, not because the triceps relaxes, but because the 
pronators do. Relaxation of the triceps does not permit of supination, although all anatomists are agreed that the biceps has supinating power. In the human biceps the principal motor force is that associated with the short or coracoid head, and the future line of evolution would appear to be along the lines of the retention of the long head or

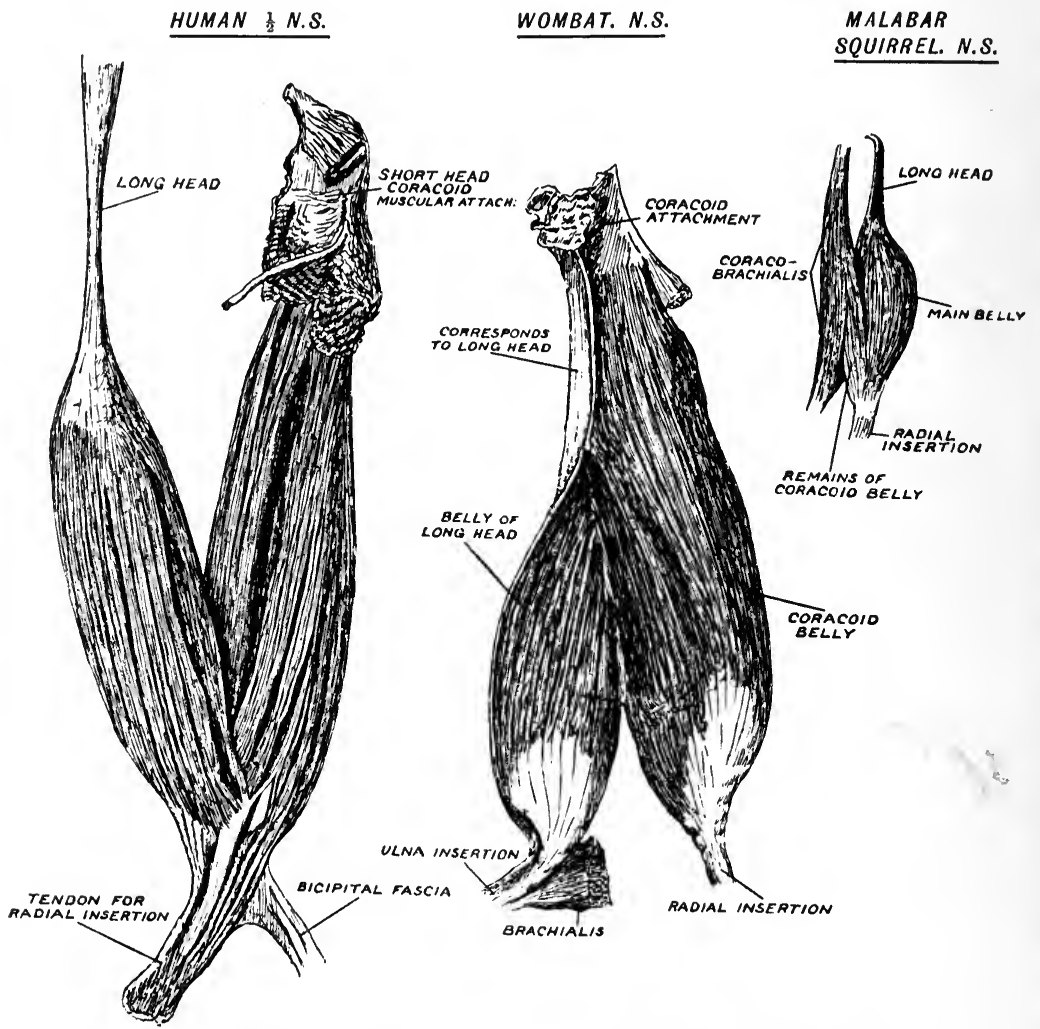

FIG. =9.-Nature's experiments with the biceps brachii.

tendon as a ligament of the shoulder joint only. It is interesting to note that in a case of infantile paralysis seen recently, the right upper limb showed a functionizing short or coracoid portion which was defined, while the long or outer portion could not be demonstrated.

In connection with the biceps, nature has made some interesting experiments. In the marsupial wombat the 
condition is as follows. At the origin there are not two separate heads, but a single broad tendon about $2.25 \mathrm{~cm}$. wide passing over the front of the shoulder joint. This is mainly attached to the coracoid, but the outer margin for about $5 \mathrm{~cm}$. is thick and tough, and is traced as in the human type to the upper rim of the glenoid cavity. There is a double insertion. The outer thick part of the tendon of origin is traced to a muscular belly $(6.5 \mathrm{~cm}$. long) which, terminating in a small tendon, is inserted into the coracoid process of the ulna together with the brachialis. The main portion of the tendon of origin is traceable to a larger muscular belly (9 $\mathrm{cm}$. long) which terminates in a longer tendon than that of the preceding, and is inserted into the tubercle of the radius. Thus in the marsupials nature clearly differentiated between flexion of the elbow and supination of the forearm.

In the Malabar squirrel (Rodentia), I found that the short or coracoid head was non-existent. Its belly arose as a slender muscle $5 \mathrm{~cm}$. wide from the middle of the belly of the coraco-brachialis and joined the main belly. This was $2 \cdot 75 \mathrm{~cm}$. wide and was connected with the long head, which resembled that of man. There was a single tendon of insertion into the upper part of the radius. I was interested and surprised to rotice this line of evolution, and proceeded to correlate this biceps with the animal's hand. I found only four digits and no opposable thumb-only a rudimentary structure, practically forming a pad, with dorsally a small nail. The hand was not destined for a higher evolution. With the specialization of biceps function in man the long head, owing to its relation with the shoulder joint, is obviously at a disadvantage in supinating. It becomes involved in joint disease and injuries to which the shoulder is liable, and is subject to displacement interfering with arm elevation. As previously stated the "selection" trend would appear to be in the direction of the retention of the short or coracoid head and belly; the long head being retained as a tendon, and its muscle belly fortifying the brachialis, as would seem to be suggested in certain of the marsupials. 


\section{Rest in Musculo-Cutaneous Paralysis}

(I) As the long head of the biceps, with which is connected a well-defined muscular belly, is related to the shoulder joint it must be rested by placing the arm in the abducted position (fig. 30), without the additional backward rotation necessary in deltoid paralysis.

(2) The elbow should be flexed to a right angle or less so as to relax and elongate the triceps. In this way we rest the brachialis.

(3) The forearm should be over-supinated so as to take strain off the biceps by elongating the pronators.

These positions are simply effected by means of the upper limb abduction splint with an over-supinated handpiece (fig. 3I). This should be worn till complete recovery has taken place. , As I have repeatedly pointed out mere flexion of the elbow is not the position of physiological rest in this form of paralysis. Failure to rest the biceps effectively is without doubt responsible for the large number of cases presenting a failure in supinating after injuries of the brachial plexus.

\section{Method of Re-education}

The ideal recovery for which we aim is such, that when the patient sits erect or stands, not only can he bring food up to the mouth (brachialis), but can supinate (biceps) so as to place it in the mouth. With the patient lying down on a table or firm bed, and with a small cushion under the head; the splint is removed and the limb placed on a pillow occupying as nearly as possible the position it occupied when in the splint (fig. 32). A sheet of carboard powdered is placed under the limb to obviate friction in movement. Thus flexion and extension take place on an even and level plane. The "moment of force" of the brachialis, i.e. the effective force, is the amount of force $(\mathrm{P})$ produced by its contraction multiplied by the perpendicular distance from its point of application to the fulcrum or elbow joint. This 


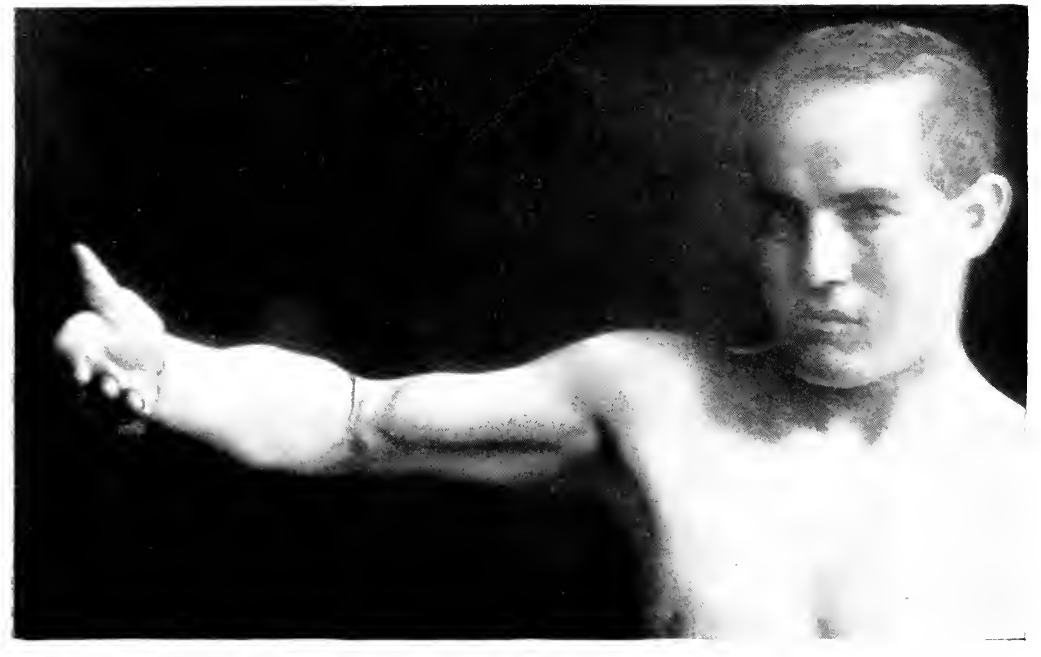

FIG. 30.-Position of rest in musculo-cutaneous paralysis.

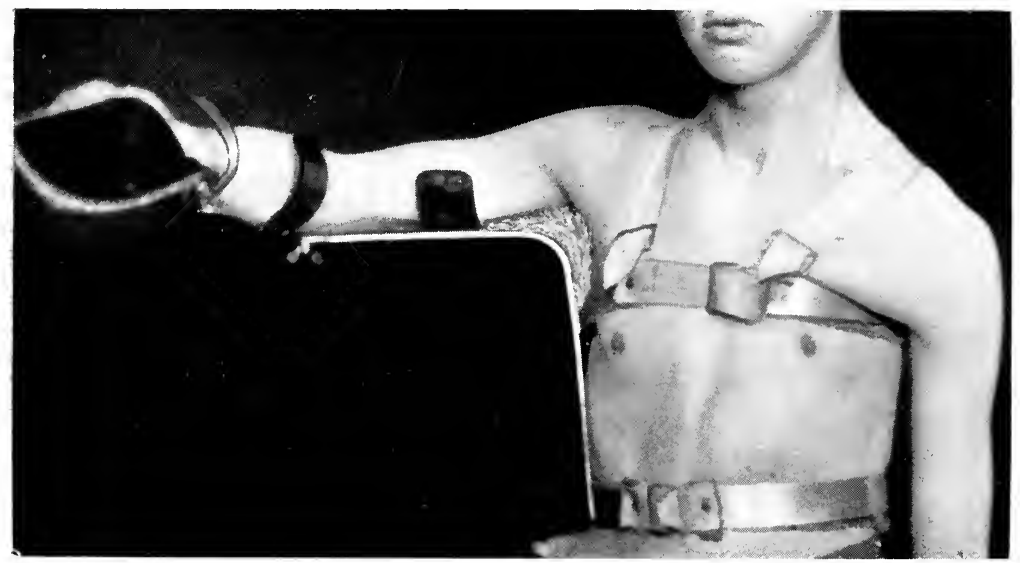

FIG. 3I.-Method of rest in musculo-cutaneous paralysis. 
, 1

'L - in 
is greatest when the forearm is at a right angle to the arm, for in this position the muscle acts more perpendicularly on the ulna (fig. 33). This action represents a lever of the third order, the power or point of insertion of the brachialis B (fig. 33) lies between the weight to be lifted $(\mathrm{W})$ and the fulcrum or centre of motion (F) in the elbow joint. In the right-angle position the perpendicular $\mathrm{B} \mathrm{F}$ (fig. 33) drawn from the line of the direction of the power to the fulcrum is greatest and becomes less, C F, E F, if for

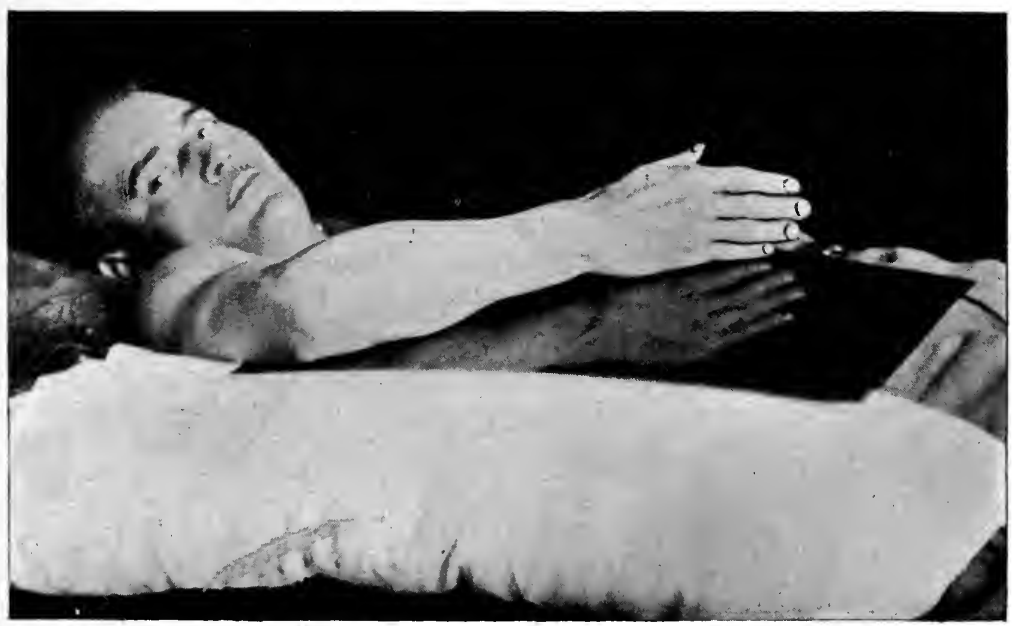

FIG. 35--Commencing re-education of the brachialis. Note the slight pronation of the hand.

example the elbow be extended as is shown in fig. 33. Thus flexion movement is most favourably commenced with the elbow at a right angle A B (fig. 34), and we move along the arc $\mathrm{A} \mathrm{C}$ to the fully flexed position. The arc of extension is gradually increased, E B and $\mathrm{H} \mathrm{B}$, till flexion can take place from full extension $\mathrm{D}$ along the arc $\mathrm{D} \mathrm{A} \mathrm{C}$. With the patient still lying flat we increase the amount of work we ask the brachialis to do by lowering the elbow, firstly by means of a smaller pillow (fig. 35), then a folded sheet, 
and finally leave it unsupported-the above procedure being successfully repeated at each stage, at first on the cardboard, and later without. During these procedures the arm is kept well adducted.
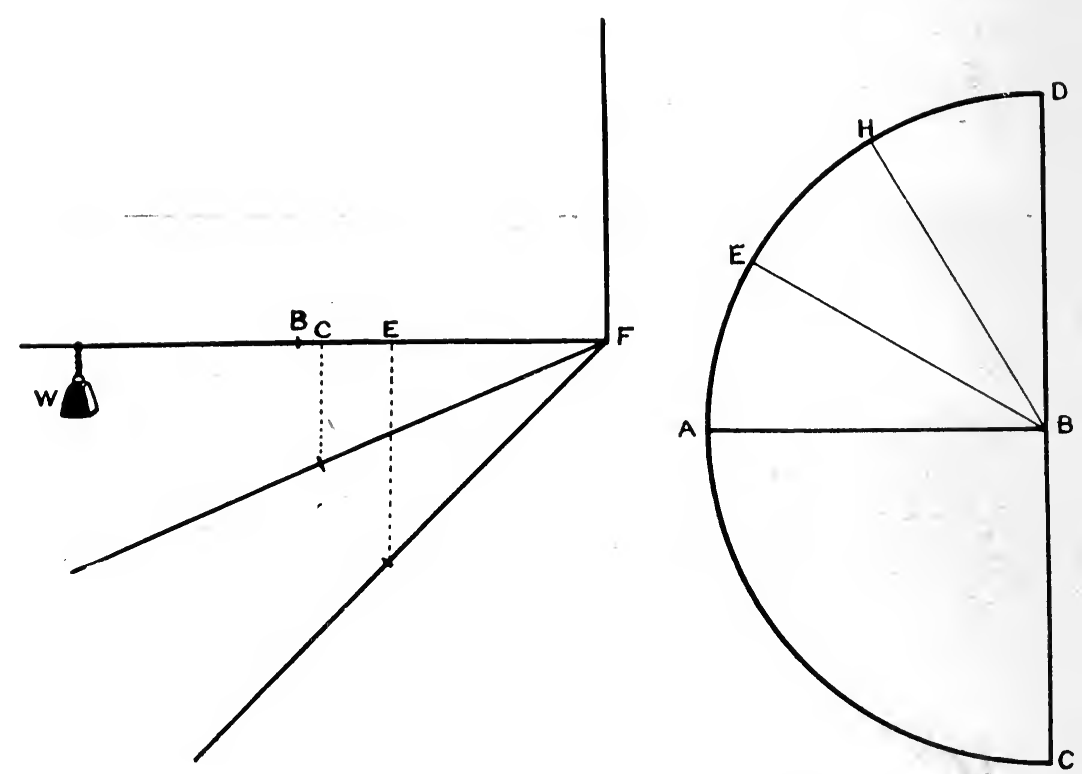

FIG. 33.-Biceps and brachialis.

FIG. 34.-Biceps and brachialis.

Having then ensured recovery in this position the patient is gradually elevated by means of pillows. The above procedure is adopted with each elevation till the erect posture is reached. Until this occurs it is best to begin each day with the lying-down position. Tested and treated in this way it is surprising what an amount of work a brachialis may perform, that for years had been regarded as hopeless for recovery.

For recovery of biceps supination the patient should lie on a table with the back of the arm resting on a firm pillow (fig. 36), and with the forearm supported by the attendant's hand, placed at a right angle A B (fig. 38). C represents 


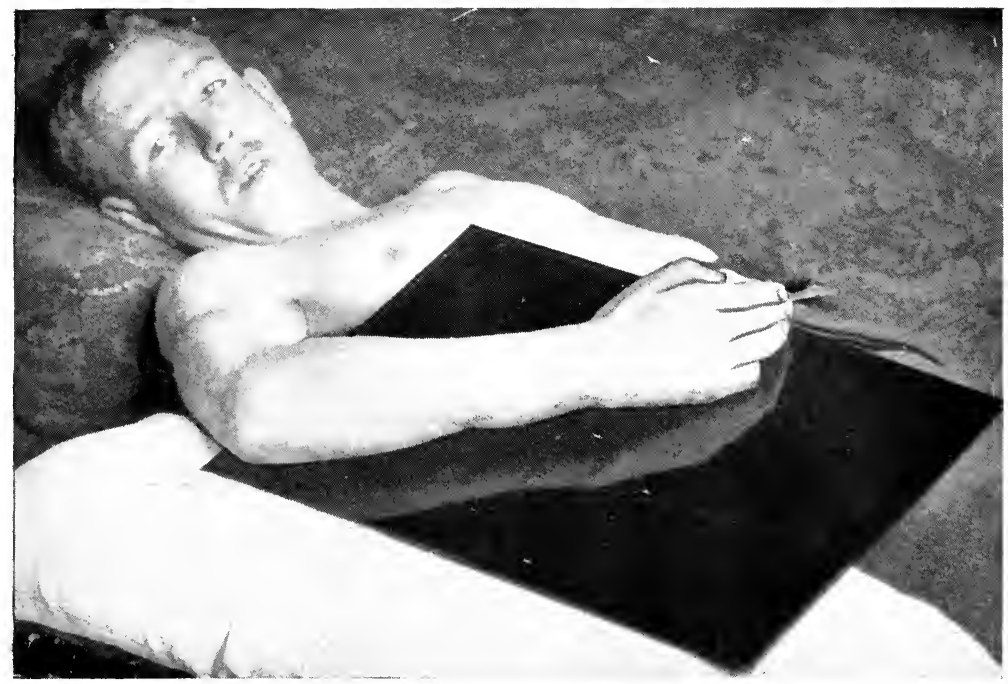

FIG. 35--Biceps and brachialis. Work for the brachialis is increased.

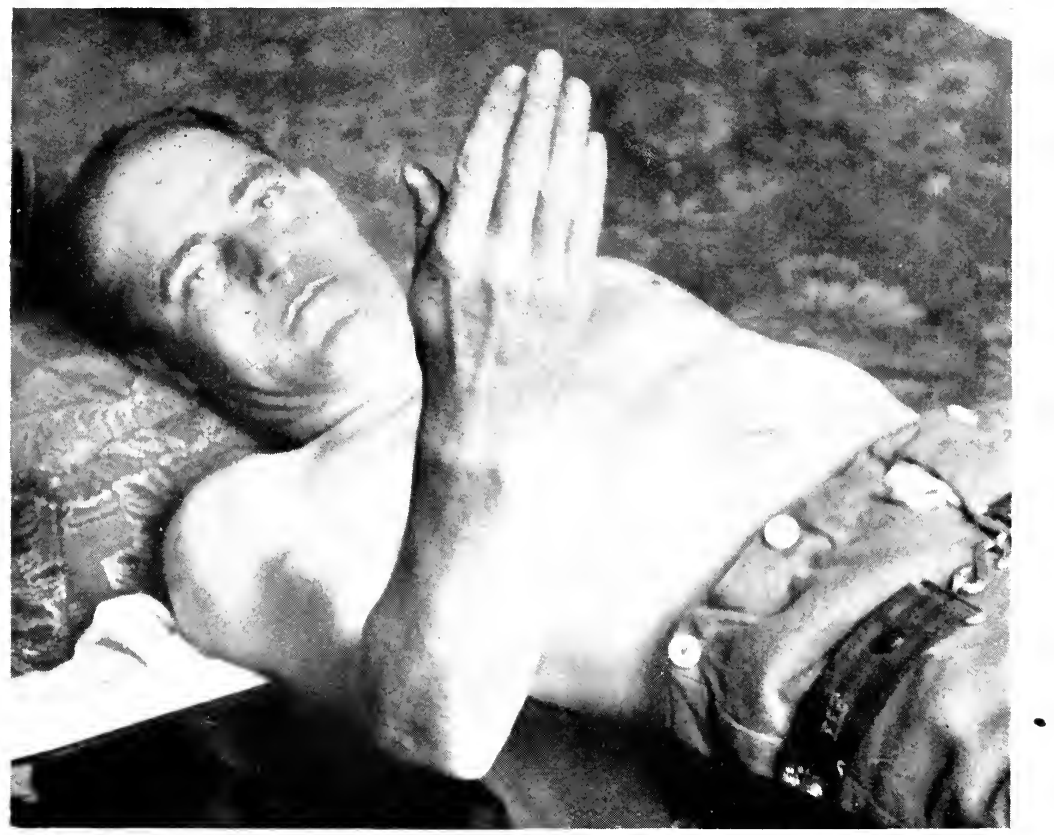

FIG. 36--Commencing re-education for biceps brachii. 
-

1

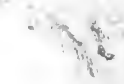


THE BICEPS BRACHII AND BRACHIALIS 83

full flexion and $\mathrm{D}$ represents full extension. If we assume $\mathrm{N}$ (fig. 37) represents the forearm in the position midway

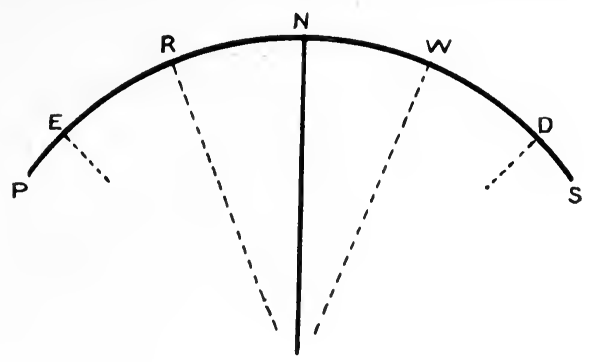

FIG. 37 .

between pronation and supination, $\mathrm{P}$ the position of overpronation, and'S the position of over-supination the arc P S would represent the maximum recovery. We place the forearm at W and coax it to supinate along the arc W S. ; then from $\mathrm{N}$ to $\mathrm{S}, \mathrm{R}$ to $\mathrm{S}$, and finally $\mathrm{P}$ to $\mathrm{S}$. (fig. 37). Having ensured, then, recovery with the arm at a right angle we lower the forearm to $\mathrm{E}$ (fig. 38), we increase the extension arc and repeat the same procedure along the plane $\mathrm{BE}$ (fig. 38). Then similarly along the planes B H and BF, till finally B D, full extension, is reached.

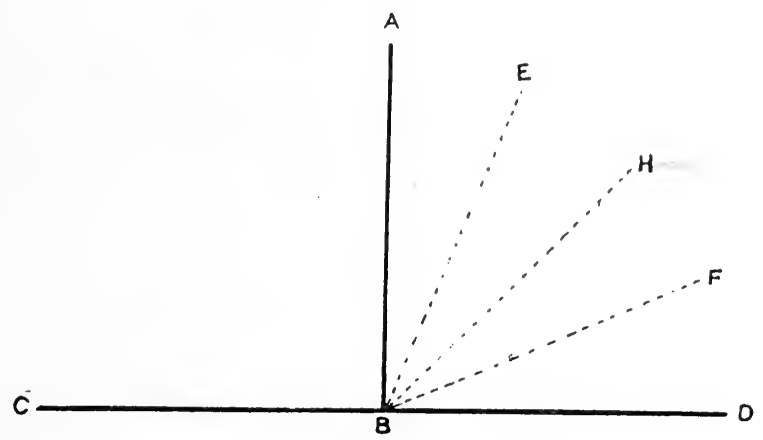

FIG. 38 .

Lengthening of the Pronator Teres.-In old cases of paralysed biceps in which failure to supinate is associated with a contracted pronator teres, it may be preferable 
to elongate the latter at once by operation than to overcome its contraction by graduated force. An attempt to strengthen supination by muscle grafting might be recommended on academic, but not on practical grounds. The distal or lower half of the muscle can be explored by making a vertical incision about two inches long over the middle of the radial side of the forearm. It has to be remembered that the brachio-radialis and the vessels and nerve lie superficial to the muscle near its insertion. I have successfully adopted this procedure on various occasions. 


\section{MEDIAN NERVE PARALYSIS}

THE movements performed by muscles supplied by the median nerve are :

(a) Pronation of the forearm. (Pronator teres and pronator quadratus.) The antagonists are biceps, supinator brevis, and supinator longus or brachio-radialis which supinate the forearm.

(b) Flexion of the wrist. (Flexor carpi radialis assisting the flexor carpi ulnaris.) The opponents are extensors carpi ulnaris, radialis longus, and radialis brevis. The palmaris longus when present acts as an accessory flexor of the wrist.

(c) Complete flexion of the index and middle fingers, third phalanx by the flexor profundus, second phalanx by the flexor sublimis, first phalanx by the lumbricales. The antagonists are the interossei which extend the second and third phalanges, and the extensor communis which extends the first or proximal phalanx.

(d) Flexion of the middle phalanx of the ring and little fingers performed by the flexor sublimis, whose antagonists are the interossei. Flexion of the proximal phalanx in these fingers is performed by the lumbricales, and of the distal phalanx by the flexor profundus (ulnar nerve).

(e) Thumb movements :

(I) Abduction of the thumb from the index finger performed by the abductor pollicis, its opponents being the adductores pollicis (ulnar nerve).

(2) Flexion of the proximal phalanx. Superficial part of flexor brevis, which is opposed by the extensor brcvis pollicis (musculo-spiral). 
(3) Rotation of the metacarpal bone on its axis, by which means the ball of the thumb is turned towards the palm and so opposition of thumb to fingers is permitted. This is performed by the opponens pollicis, and the opponent is the long abductor pollicis (extensor ossis) (musculo-spiral nerve).

(4) Flexion of the distal phalanx. Flexor longus pollicis. The opponent is the extensor longus pollicis (musculo-spiral).

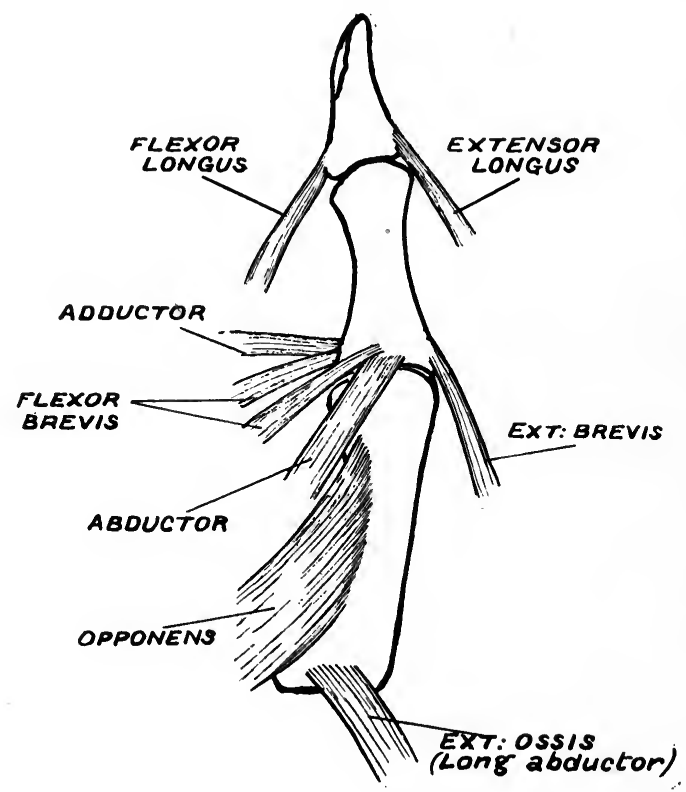

FIG. 39.-The motor forces which move the thumb.

The extensors longus and brevis and the long abductor are supplied by the musculo-spiral nerve. The opponens, abductor brevis and outer head of flexor brevis, by the median nerve, as well as the flexor longus. The adductor and inner head of flexor brevis are supplied by the ulnar nerve.

The Pronators.-The two muscles producing rotation inwards, or pronation of the radius, are the pronator teres and pronator quadratus. The position of flexion of the elbow is most favourable for pronator teres action, and the position of extension for that of the quadratus. Both muscles however can act in either the position of an extended or bent elbow. Our first but erroneous impression would appear 
to be that these pronators are scarcely powerful enough to antagonize the supinators. The biceps seems a much more powerful muscle than the pronator teres. The biceps however, like the brachio-radialis (supinator longus), is an elongate muscle with a large amount of tendon in its composition. A cord six inches long is more easily broken than a cord of the same thickness and half the length. The biceps has its action weakened also owing to its long head passing through the shoulder joint. In the dissection of an adult male forearm the pronator is a fleshy somewhat rounded muscle from its beginning almost to its insertion. It measures about $\mathrm{I} 7 \mathrm{~cm}$. long, and seen anteriorly its width is $2.5 \mathrm{~cm}$. The pronator quadratus is also a muscular muscle with a width of about $5 \mathrm{~cm}$. Loss of pronation alone would be sufficient to ruin the utility of the upper limb. Food could not be grasped owing to the inability to rotate the radius inwards and the palm of the hand downwards.

Thumb Opposition. - The importance of the action of the opponens pollicis cannot be too strongly emphasized. Its loss is sufficient to ruin the utility of an otherwise perfect upper limb. The power of opposing the thumb to the fingers, necessary for the finer movements of grasping, reaches its highest developments in man. In machine accidents to the hand every effort is made by the wise surgeon to avoid amputation of the thumb. Fingers without an opposable thumb are of little use. The retention of the thumb would be preferable to the loss of the middle, ring, and little fingers. The thumb represents one blade of a dissecting forceps.

Flexor Profundus and Flexor Sublimis. - The arrangement of the tendons of these muscles in the hand illustrates one of nature's methods for giving strength to muscle near its insertion. As the root of the finger is approached the tendon of the sublimis (superficial) is grooved below for the deep flexor. About the middle of the proximal phalanx the tendon of the sublimis divides into two slips, and between the two the profundus tendon passes, i.e. the sublimis rests 
on the profundus. Then the two portions of the sublimis reunite below the profundus, forming a grooved channel on which the deep tendon rests. Finally the sublimis divides again into two slips, each being inserted into the sides of the middle phalanx, while the profundus tendon

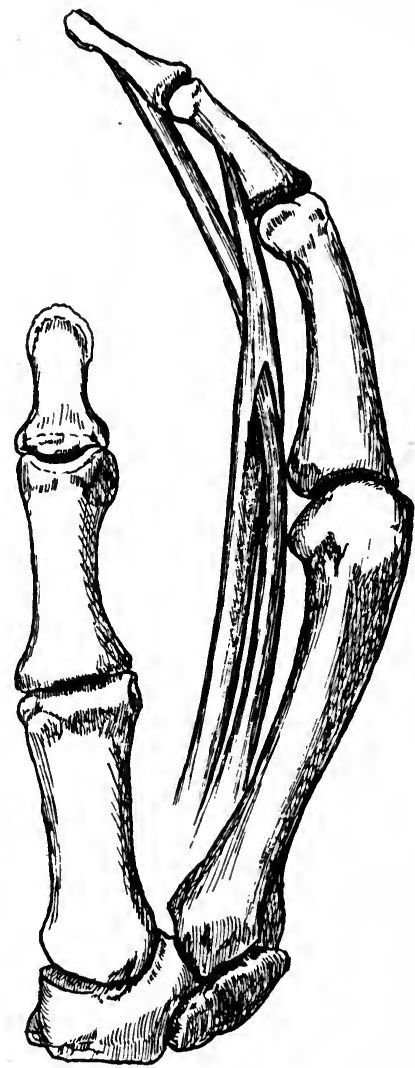

FIG. 40.-Reciprocal leverage by the profundus and sublimis to improve flexion power.

is inserted into the base of the distal phalanx. By means of mutual support, first of the sublimis on the profundus and then of the profundus on the sublimis, improvement of leverage is effected (fig. 40). These are the muscles of coarse flexion, and are antagonized by the interossei, which 
are comparatively short muscles having a powerful muscular grip of origin from the metacarpal bones. The interossei do not have to accommodate themselves to movements of the wrist and elbow like their antagonists.

Lumbricales. -These are usually four in number, small and round, and were so named owing to their resemblance to the earth worm. Those for the index and middle fingers are supplied by the median nerve and for the ring and little fingers by the ulnar nerve. They are fleshy in character from their origin almost to their insertion, and are instances of muscles connected at both insertion and origin with the tendons of other muscles. They arise in the palm of the hand from the tendons of the flexor profundus. From their origins they are traced to the radial or outer sides of the metacarpo-phalangeal joints of each finger and on to the outer side of the proximal phalanx. At about the junction of the middle and proximal thirds each is traced to the corresponding interosseous tendon and has an attachment to the phalanx as well. The action of the lumbricales is to produce flexion at the metacarpo-phalangeal articulations. They antagonize the extensor communis, whose action is to extend at the metacarpo-phalangeal joints (fig. 54, p. I09). Flexion of a phalanx is produced in one of two ways: either by a pull on the palmar surface of the phalanx (profundus and sublimis), or by a pull on the dorsal surface by a muscle whose motor machine lies on the front of the hand (lumbrical). Owing to the tendons of the sublimis and profundus passing on the front of the proximal phalanx, a palmar attachment of the lumbrical was not possible. The passage of the lumbrical along the side of phalanx is a wise provision of nature to secure unimpeded action. Furthermore, the phalanx forms a rigid support by means of which the action of the interosseous as well as of the lumbrical is strengthened.

Compared with the profundus and sublimis the lumbricales are the muscles of fine flexion and move the fingers with great rapidity. They are the muscles mainly concerned in the quick movements of the fingers seen in 
musicians, and for that reason were known to Cowper some centuries ago as the musculi fidicinales. In playing the piano we relax the interossei so as to produce slight flexion (sublimis) at the first interphalangeal joints-the rapid movements of the fingers being performed at the metacarpophalangeal joints, flexion by the lumbricales and extension by the extensor communis. The power has been ascribed to the lumbricales, because they are connected with the tendons of the interossei, of extending the middle and distal phalanges in addition to flexing the proximal phalanges. The direction of their pull on the proximal phalanx is from the front and not dorsally. In a dissection of a fresh cadaver, traction made on the lumbricales produces flexion at the metacarpo-phalangeal joints only, but makes no impression on flexed interphalangeal joints. That the lumbricales do not extend the middle and distal phalanges is also shown by the following two simple tests.

(I) If we acutely flex the two interphalangeal joints the lumbricales, if they extended these, would be relaxed and elongated to allow the flexor profundus and flexor sublimis to act, yet in spite of this flexion we can readily bend the metacarpo-phalangeal joints. This is performed by the lumbricales, and the relaxation at the interphalangeal joints by the interossei.

(2) If we extend at the metacarpo-phalangeal joints, keeping the middle and distal phalanges bent, the lumbricales are in a state of physiological relaxation and elongation to allow of contraction and shortening of the extensor communis. Yet we can (action of the interossei) extend the middle and distal phalanges from acute flexion. If the lumbricales were extensors of these, as is so frequently taught, then they would be in a physiological state of relaxation and contraction at one and the same time-relaxed to allow of extension at the metacarpo-phalangeal joints by the communis, and contracted to produce extension at the interphalangeal joints. 


\section{Anatomical Rest in Median Nerve Paralysis}

(I) Owing to the fact that the long flexor muscles arise above the elbow joint this should be placed in the position of flexion.

(2) The forearm should be over-pronated to relax and elongate the biceps and supinators, thus resting the pronators teres and quadratus.

(3) The wrist should be flexed so as to rest the affected flexor carpi radialis.

(4) The thumb should be flexed at the interphalangeal and

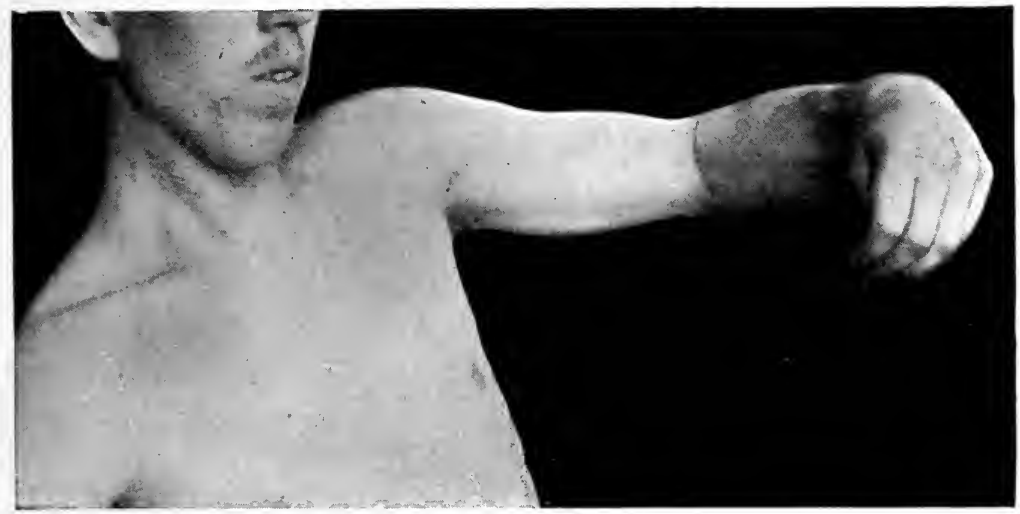

FIG. 4I.-Position of rest in median nerve paralysis.

metacarpo-phalangeal joints, thus elongating the opposing long and short extensors supplied by the musculo-spiral nerve, and should be drawn across the palm of the hand to rest the opponens and prevent. overaction of the long abductor pollicis (extensor ossis). These positions of the thumb can be easily effected by means of a strip of adhesive plaster (fig. 46). The failure to recognize that the opponens represents one of the most important muscles in the upper extremity is responsible for the inutility of the upper limb which is frequently seen in old cases of infantile paralysis. This is owing to the fact that the patient is unable to oppose the thumb to the fingers. An upper limb may have the rest 
of its musculature perfect, but be rendered functionless owing to the loss of the opponens pollicis.

(5) As regards the index and middle fingers, these should be bent, but not acutely; at the interphalangeal and metacarpo-phalangeal articulations, since all the flexors are affected, the profundus acting on the distal phalanges, sublimis on the middle, and the lumbricales on the proximal ones. In the case of the ring and little fingers, flexion at the interphalangeal and metacarpo-phalangeal joints is also advisable, although the sublimis acting on the middle phalanges is the only muscle affected.

Although I regard the interossei of the index and middle fingers as receiving in addition to branches of the ulnar some accessory branches from the median nerve, this would not alter the above disposition of the fingers for purposes of rest.

In a case with combined ulnar and median affection the positions would be similar to the above as regards the elbow, wrist, and thumb. In the fingers a difficulty arises, owing to the fact that we are dealing with two opposing and weakened groups at the interphalangeal joints, namely the interossei and flexors profundus and sublimis. The best position is good flexion at the metacarpo-phalangeal joints, so as to rest the lumbricales and relax and elongate the extensor communis, and slight flexion at the interphalangeal joints. As the flexors recover before the interossei we must be careful not to allow flexor contraction to occur, and thus interfere with the recovery of the interossei. Functional recovery of the groups should be pari passu; this ideal may be obtained by careful muscle re-education.

In a case with combined ulnar and musculo-spiral affection the wrist should be extended (dorsi-flexed), since although the flexor carpi ulnaris is affected, we have intact for flexion the flexor carpi radialis ; and furthermore, flexion is more readily recovered than extension. The thumb should be adducted towards the index finger, with extension at the metacarpo-phalangeal and interphalangeal joints. As the flexor sublimis (median nerve) is intact in the ring and little fingers, and all the flexors in the index and middle 



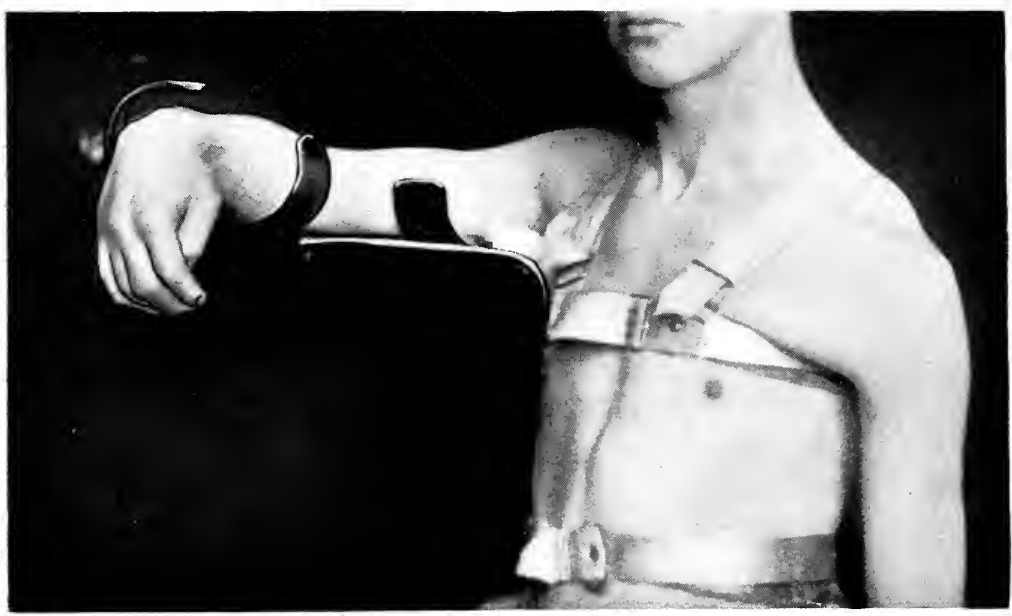

FIG. 42.-Method of effecting rest in median nerve paralysis.

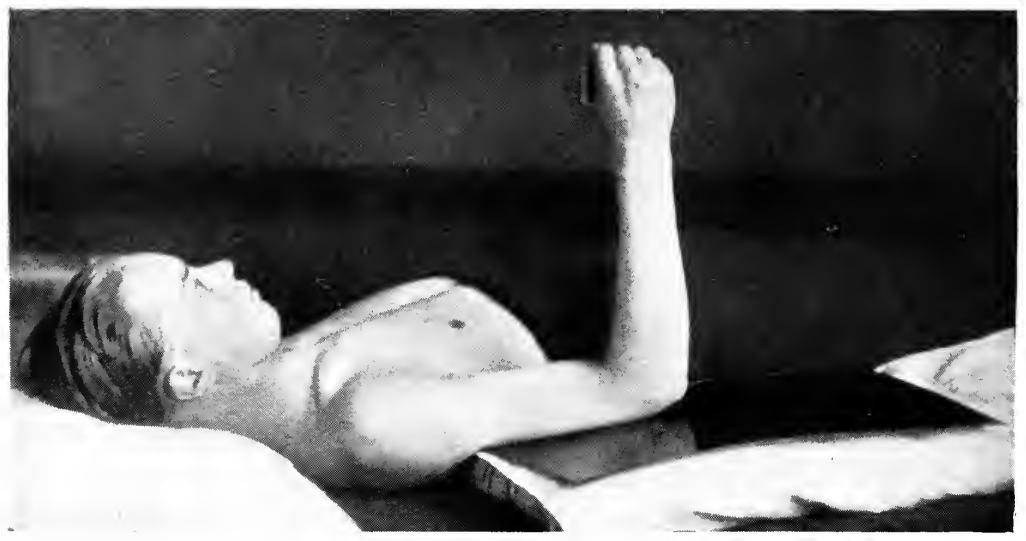

FIG. 43.-Commencing re-education of pronation. Median nerve paralysis 
fingers (median), the best position for the fingers is extension both at the metacarpo-phalangeal joints to rest the extensor communis, and at the interphalangeal joints to rest the interossei.

In a case with combined median and musculo-spiral affection the wrist should be extended (dorsi-flexed), since although the flexor carpi radialis is affected we have intact for flexion the flexor carpi ulnaris ; and furthermore, flexion, as stated above, is more readily recovered than extension. The thumb should be placed across the palm of the hand, as the opponens is the most important thumb muscle. This means also flexion at the metacarpo-phalangeal joint, but the interphalangeal joint should be extended, as recovery of the long extensor is more difficult than that of the long flexor. In the case of the fingers, the flexor sublimis, passing to the middle phalanges of all, is affected, as well as the lumbricales and profundus of the index and middle fingers. As regards position the lumbricales must be sacrificed to the extensor communis. The most suitable position is flexion at the interphalangeal joints of all the fingers, to rest the flexors profundus and sublimis, with hyper-extension at the metacarpo-phalangeal joints to rest the extensor communis. All these positions can be affected by means of the upper limb abduction splint, with the hand-piece adjustable to suit the different positions of flexion and extension or pronation and supination, and with an adjustable hinge opposite the elbow joint.

\section{Re-education in Cases of Median Paralysis}

Pronation.-For recovery of pronation the patient should lie on a table with the back of the arm resting on a firm pillow (fig. 43) and with the forearm, supported by the attendant's hand, placed at a right angle A B (fig. 45). $\mathrm{C}$ would represent full flexion and $\mathrm{D}$ full extension.

If we assume $\mathrm{N}$ (fig. 44) represents the forearm in the position midway between pronation and supination, $\mathrm{P}$ the position of over-pronation, and $\mathrm{S}$ the position of over- 
supination, the arc S P would represent the maximum recovery, or roo units of work.

We place the forearm at $R$ and coax it to pronate along the arc $\mathrm{R} \mathrm{P}$, then from $\mathrm{N}$ to $\mathrm{P}, \mathrm{W}$ to $\mathrm{P}$, and $\mathrm{S}$ to $\mathrm{P}$ (fig. 44).

Having ensured, then, recovery with the arm at a right

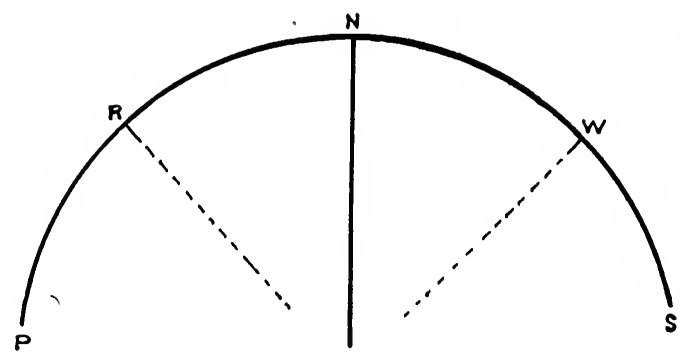

FIG. 44.

angle we lower the forearm to $\mathrm{E}$ (fig. 45), thus increasing the extension arc and repeat the same procedure along the plane B E (fig. 45). Then, similarly, along the planes B H, and B F, till finally $\mathrm{BD}$, or full extension, is reached. The patient is

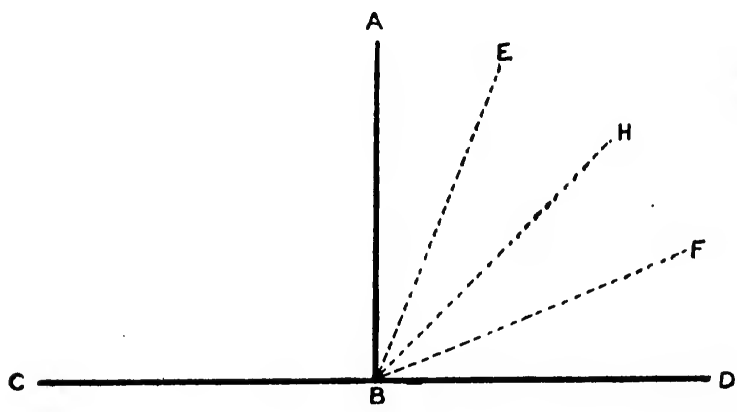

FIG. 45 .

gradually elevated by means of pillows and these procedures successively repeated till the erect posture is reached.

Wrist Flexion.-Flexion at the wrist joint, though impaired, is not lost, as the flexor carpi ulnaris is intact. We stimulate the return of function in the flexor carpi radialis by commencing work in the over-pronated position $\mathrm{P}$ 



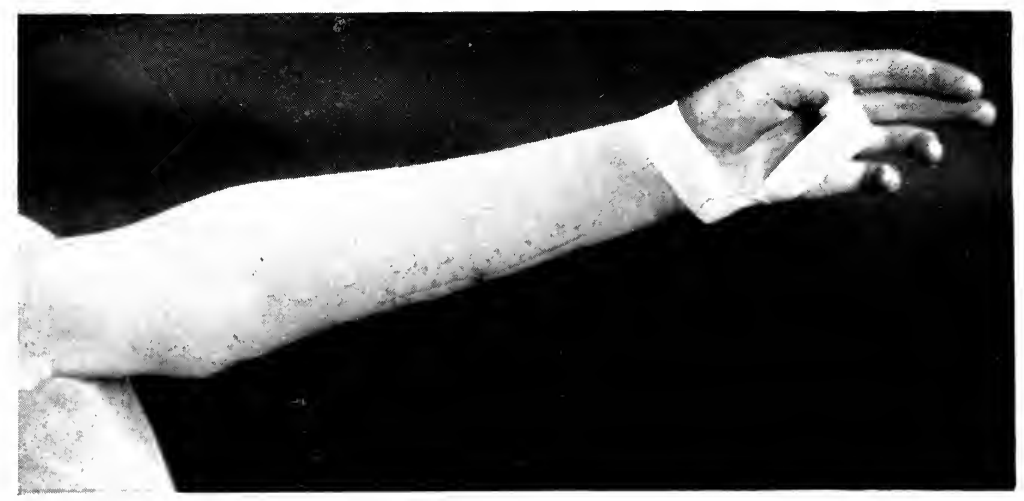

(a)

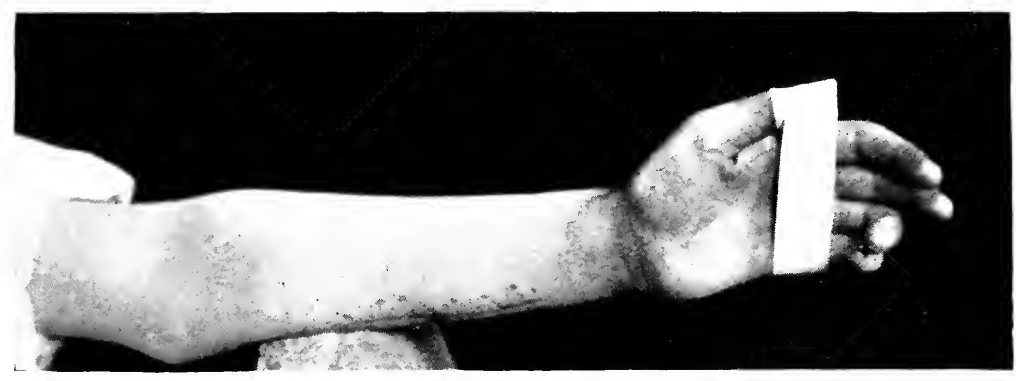

(b)

FIG. 46.-Method of resting $(a)$ and of re-educating $(b)$ the thumb in median nerve paralysis.

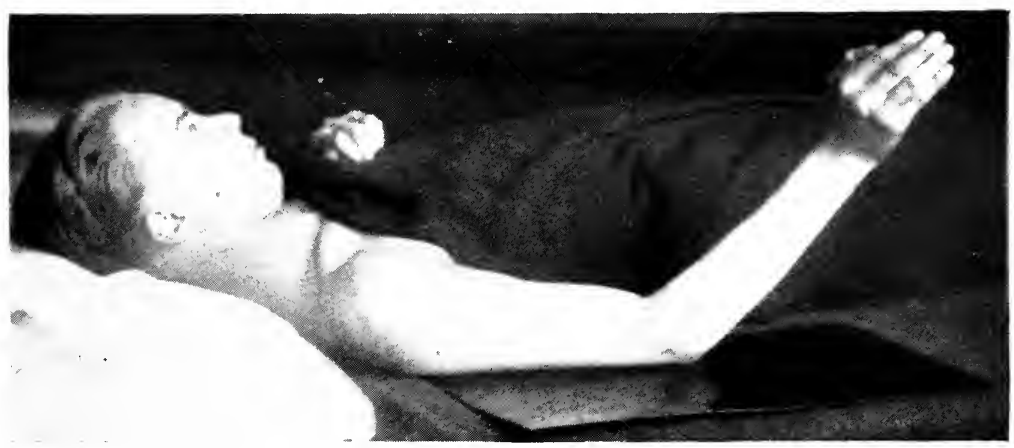

FIG. 47.- Re-educating finger flexion. 
(fig. 44), with the dorsum of the hand uppermost and the wrist extended so as to improve the leverage action of the carpal flexors; and gradually work towards the mid prone and supine position $\mathrm{N}$, and finally to the over-supinated position S. Flexion of the wrist is most difficult with the palm of the hand looking upwards.

Thumb Opposition and Flexion. - With the hand in the position midway between pronation and supination, the opponens pollicis and flexor brevis pollicis are stimulated to action by relaxing slightly the adhesive strapping in order to permit of abduction, and then encouraging the patient to oppose the thumb to the fingers. The abduction is gradually increased (fig. 46) till we can obtain opposition from full abduction. The adhesive strapping should not be removed completely till recovery of opposition takes place, as the powerful abductor longus (extensor ossis) and the extensor brevis will produce forcible abduction and extension of the thumb. During the varying degrees of increasing abduction of the thumb, the flexor longus pollicis is stimulated by grasping the proximal phalanx and asking the patient to flex and extend at the interphalangeal joint.

Finger Flexion.-Although in the case of the ring and little fingers the sublimis alone is affected it is preferable to work all the fingers together. Flexion of the fingers is best stimulated by resting the arm on a firm pillow with the patient lying down. The elbow is flexed to a right angle and the wrist extended. Extension of the wrist improves leverage by providing a pulley over which the weakened long flexors can act (fig. 48). In this way the "moment of force" of the flexors is increased. Slight extension of the fingers is at first allowed and the patient encouraged to bend them. The extension is gradually increased (fig. 47) till we can flex both at the interphalangeal and metacarpo-phalangeal joints from full extension. We should commence, as in the case of the carpal flexors, with the hand in the over-pronated position, and gradually work towards the mid prone and supine position (fig. 47), and finally to the over-supine position. Flexion of the fingers, 
as in the case of the wrist, is easiest with the palm of the hand looking downwards.

In some cases we may have complete recovery of the profundus for the index and middle fingers and of the sublimis for all the fingers, but the lumbricales for the index and middle fingers may be delayed in recovery. Hyper-

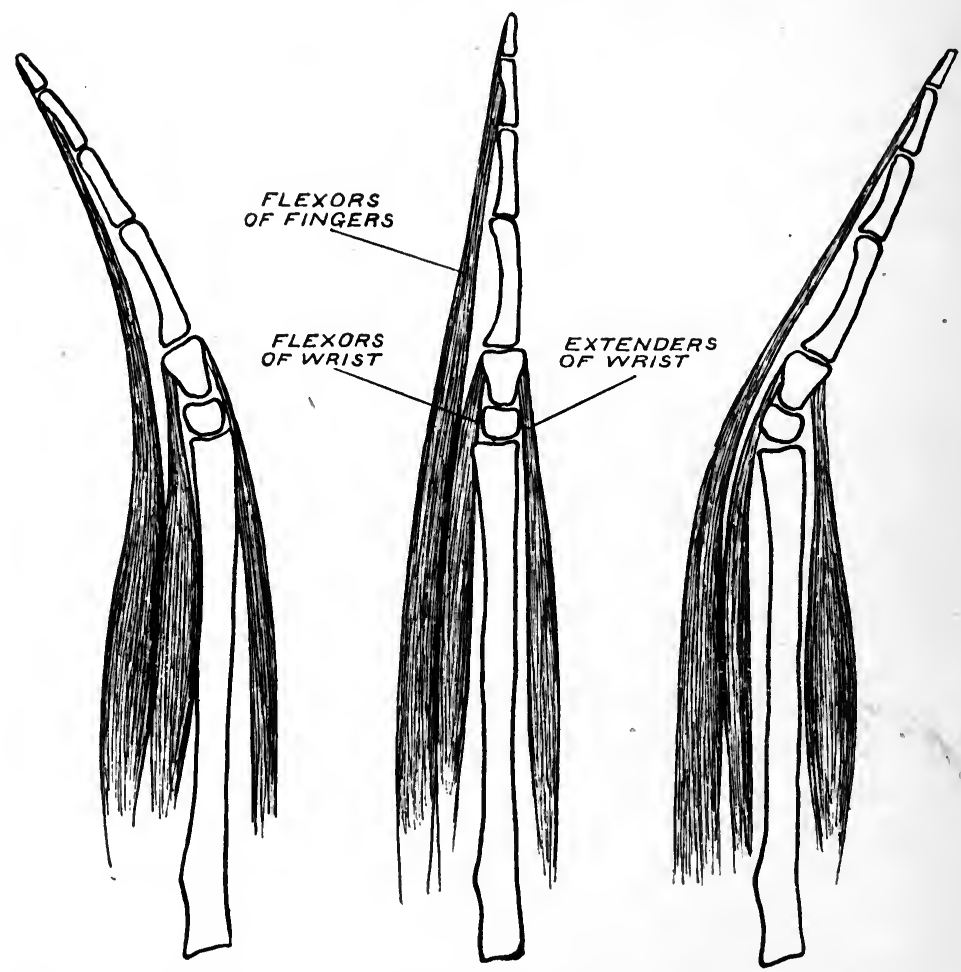

FIG. 48.-How flexor leverage of the fingers is improved by extending (dorsi-flexing) the wrist.

extension may then occurat the metacarpo-phalangeal joints, due to over-action of the extensor communis. This is treated by acutely flexing on a splint the index and middle fingers at the metacarpo-phalangeal joints, and encouraging pure lumbrical action by asking the patient to flex at these joints, whilst the interphalangeal joints are extended by means of light straight metal splints. 


\section{CHAPTER V}

\section{ULNAR PARALYSIS}

THE ulnar nerve arises from the inner cord of the brachial plexus-its origin being traced to the eighth cervical and first thoracic (or dorsal) nerves. There are two sets of muscular branches, namely:

(I) Those which arise in the forearm.

(2) Those which arise in the palm of the hand.

(I) BRanches Which aRISE IN THE FoRearm

These are given off as a rule immediately the nerve enters the forearm and supply:

(a) The flexor carpi ulnaris, which is a flexor of the wrist and accessory ulnar abductor of the hand, and $(b)$ the inner half of the flexor profundus digitorum, which produces flexion of the distal phalanges of the ring and little fingers. It is important to note that the origin of the flexor carpi ulnaris can be traced above the elbow joint to the inner or medial condyle, while the profundus arises below the joint-its origin being from the upper two-thirds of the front of the ulna as well as from the interosseous membrane.

(2) Branches which aRise in the Hand

These supply:

(I) Small Muscles of the Little Finger, viz.-(a) Abductor, which passes to the ulnar side of the base of the proximal phalanx and abducts the little finger from the mid line, separates the little finger from the ring finger. 
(b) Flexor brevis also passes to the ulnar side of the proximal part of the first phalanx and acts like the preceding as an abductor of the little finger. Both these muscles are said to have flexion power at the metacarpo-phalangeal joint.

They are however only abductors, since we can separate the little finger from the ring if the metacarpo-phalangeal joint be in the position of extension.

(c) Opponens or Adductor.-This is inserted into the metacarpal bone of the little finger on the inner or ulnar side. Its function is to antagonize or oppose the little finger to the others, and it resembles in action the opponens of the thumb. By its contraction the hollow of the palm of the hand is deepened, and so we have formed what the older anatomists called Diogenes' Cup.

(2) Small Muscles of the Thumb, viz.-(a) Adductores pollicis (obliquus and transversus). Both these portions have a tendinous insertion into the inner or mesial side of the base of the proximal phalanx of the thumb. By their action the thumb is approximated inwards towards the index finger.

(b) Deep portion of the flexor brevis pollicis. This portion is inserted into the ulnar side of the base of the first phalanx of the thumb. It is a bender of the proximal phalanx of the thumb (fig. 39).

(3) Two inner Lumbricales (third and fourth).--These pass to the radial side of the ring and little fingers respectively and bend the metacarpo-phalangeal joints of these digits, and thus are the antagonists of the extensor communis tendons acting on these joints.

(4) All the Interossei (both palmar and dorsal).-These fill the spaces between the metacarpal bones, and from their positions are divided into two sets, dorsal and palmar. These are the muscles that extend the middle and distal phalanges, being the antagonists of the flexor sublimis and flexor profundus ; and they also draw the fingers out or abduct and draw them in or adduct. They do not flex at the metacarpo-phálangeal articulations. This is performed by 
the lumbricales. The dorsal interossei or abductors arise from the sides of adjacent metacarpal bones forming each interosseous or interbony space. Thus they are four in number. The first (sometimes called abductor indicis) lies between the thumb and index finger, the second between the index and middle, the third between the middle and ring, and the fourth between the ring and little fingers.

Taking the mid line as a longitudinal line drawn through the middle of the mesial finger, these muscles are abductors, draw the fingers out from the mid plane, and so their attachment can easily be remembered. Thus we can abduct or draw our index finger out towards the thumb, and to secure this pull the tendon of the first dorsal interosseous passes over the metacarpo-phalangeal joint on its radial side. The middle finger can be moved (abducted) either towards the index or ring fingers, and so we find that the dorsal interossei between the index and middle and between the middle and ring fingers pass on either side of the middle finger. To abduct the ring finger, i.e. draw it from the mid line towards the little finger, we find that the dorsal interosseous between the ring and little fingers passes to the ulnar side of the ring finger. Both the thumb and little finger have independent abductors. For the thumb we have the abductor pollicis (median nerve) and the long abductor (musculo-spiral nerve). For the little finger we have the abductor minimi digiti (ulnar nerve).

There thus only remain for consideration the muscles that adduct or move towards the mid plane, the index, ring, and little fingers, as the middle finger can be abducted either towards the ulnar or radial side by the dorsal interossei. The thumb has its own intrinsic adduction. This adduction of the fingers is performed by means of the palmar interossei. They are three in number, and smaller than the dorsales. The fingers naturally incline towards the mid line, and a greater muscular effort is required for abduction than for adduction. The pull would obviously be on the ulnar side 
of the index and on the radial side of the ring finger and little fingers. The muscle to pull in the index finger arises from the ulnar side of its metacarpal, and those for the ring and little fingers arise from the radial sides of their respective metacarpals. Extension of the proximal phalanges of the fingers is performed by the extensor communis tendons, and of the middle and distal phalanges by the interossei. Flexion of the distal phalanges is performed by the flexor profundus, and of the middle phalanges by the flexor sublimis. Flexion of the proximal phalanges (metacarpo-phalangeal) is performed by the lumbricales. The lumbricales do not assist in extending the two distal phalanges. The interossei do not assist in flexing at the metacarpo-phalangeal articulation. In reference to the action of the interossei the following four simple tests are important:

(a) If we produce acute flexion at the interphalangeal joints (relaxation of the interossei and contraction of the flexor profundus and sublimis) and over-extension at the metacarpo-phalangeal joints (extensor communis), we can flex at the metacarpo-phalangeal joints from over-extension. This is performed by contraction of the lumbricales.

(b) We can extend at the interphalangeal joints as readily with extension as with flexion of the metacarpo-phalangeal joints. If the interossei were flexors of these joints, then, in the state of extension they would be in a physiological state of relaxation, and so could not contract in order to produce extension at the interphalangeal joints.

(c) If we flex at the first or proximal interphalangeal joint, i.e. relax the interossei, the distal phalanx is quite flaccid on tapping. Its extension power is lost. Perform the same movement at the metacarpo-phalangeal joint of the thumb and we find that flexion here does not affect the distal phalanx. Its extensor is a separate muscle, the extensor longus pollicis.

(d) Abduction and adduction of the fingers take place with extension of the two distal phalanges. If we flex the interphalangeal joints, relax the interossei, these actions 
are interfered with; and this is very marked if we prevent the action of the extensor communis by flexing at the metacarpo-phalangeal articulation. If we extend the fingers and hold the index and middle steady we can abduct or adduct the ring finger with ease if it be extended, but flex at the interphalangeal joints and these movements are lost. Abduction or adduction is with extension the result of interosseous muscle contraction. If we take for example the movement of abduction of the ring finger, extension of the distal phalanges would depend on the contraction of the abductor or dorsal interosseous, since the adductor or palmar interosseous would be relaxed to admit of the movement.

The long tendons of the interossei are placed lateral to the proximal phalanges which give them an attachment. Traction on these would be lateral (abduction or adduction). These tendons are connected on the dorsum of the proximal phalanges to the extensor communis tendons by fibrous membrane-thin proximally, thicker more distally. The motor engines (muscles) of the interossei have no direct pull on the common tendons. The interossei tendons cross the first inter-phalangeal joints laterally, then pass to the dorsum of the middle phalanges which give them an attachment-the tendons of each side having a strong fibrous connection. The tendons converge and are attached together on the dorsum of the proximal parts of the distal or third phalanges (fig. 56, p. II5).

\section{Anatomical Rest in Cases of Ulnar Paralysis}

(I) As the flexor carpi ulnaris arises above the elbow joint flexion of the elbow is preferable, and this can be simply effected by means of a sling, but best by the abduction arm splint.

(2) The wrist joint should be flexed (palmar flexion) to take all strain off the flexor carpi ulnaris, and the hand should be diverted towards the ulnar side to prevent radial deviation. 
(3) The thumb should be brought across the palm of the hand by means of a strip of plaster, so as to rest the flexor brevis and adductores pollicis (fig. 46). The importance of this cannot be too strongly insisted on, since failure to adduct means an impairment of opposition of thumb to fingers.

(4) In resting the fingers we have to consider the two inner tendons of the flexor profundus or benders of the distal phalanges of the ring and little fingers at the second or distal interphalangeal joint; all the interossei or extensors of the two distal phalanges of all the fingers; and the two inner lumbricales or benders at the metacarpo-phalangeal

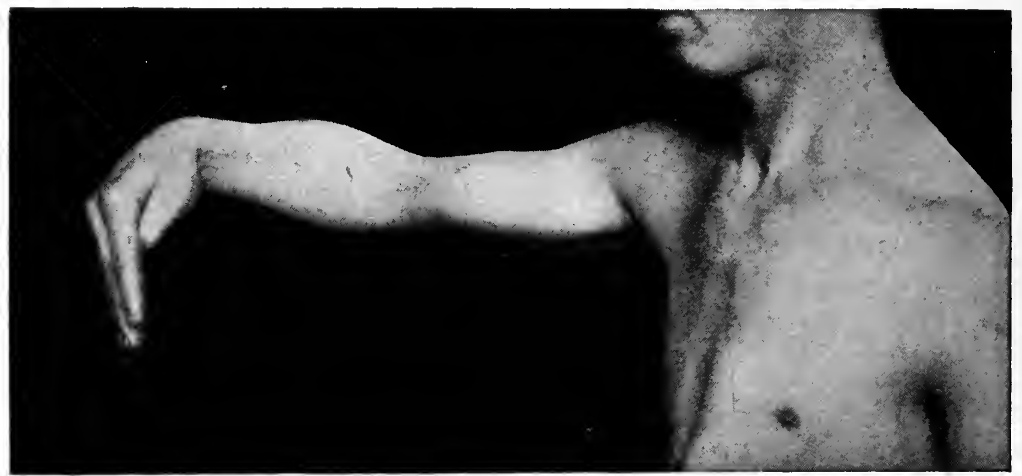

FIG. 49.-Position of rest for the upper extremity in ulnar paralysis.

joints of the little and ring fingers. The lumbricales are rested by flexing the fingers at the metacarpo-phalangeal joints; it is preferable to rest all four, although only the inner two are affected. In the case of the index and middle fingers rest of the interossei is secured by extension at the interphalangeal joints, as we have no profundus to be considered. In the case of the ring and little fingers we have to choose between rest of the profundus by bending at the distal interphalangeal joint, which means elongating the interossei, or extending at the interphalangeal joints so as to rest the interossei.

As the sublimis is normal (median nerve) in the ring and little fingers, and as we are resting other benders, viz. the 
lumbricales, there should be no hesitation in regarding the interossei as suitable for rest in preference to the flexor profundus. In any case of ulnar paralysis it is not any failure to bend, but the failure to extend at the two interphalangeal joints that causes difficulty. The positions for rest in ulnar paralysis then would be :

(I) Flexion at elbow.

(2) Palmar flexion of the wrist.

(3) Thumb adducted across the palm of the hand.

(4) Fingers bent at the metacarpo-phalangeal joints, and extended at the interphalangeal joints.

(5) The hand midway between pronation and supination.

These positions can be effected by means of the upper limb abduction splint. This is hinged at the elbow, flexion being adjusted by means of a screw or other mechanism. Adjustments can also be provided for the hand-piece by means of which flexion and extension of the wrist and pronation and supination of the forearm are regulated. With recovery of flexion the wrist is extended to the mid position, and similarly with the metacarpo-phalangeal joints. This form of splint is best worn till recovery has taken place.

\section{Methods of Re-education in Ulnar Paralysis}

Flexion at the wrist joint, though impaired, is not lost, as the flexor carpi radialis is intact. We stimulate the return of the function in the flexor carpi ulnaris by commencing work in the over-pronated position $\mathrm{P}$ (fig. 50), the dorsum of the hand being uppermost and the wrist dorsiflexed. This improves the leverage action of the carpal flexors, and we gradually work through $\mathrm{F}$ and $\mathrm{R}$ towards the mid prone and supine position $\mathrm{N}$, and finally to the oversupinated position $\mathrm{S}$ through $\mathrm{D}$ and $\mathrm{W}$. Flexion of the wrist is most difficult with the palm of the hand looking upwards.

Re-education to regain ulnar abduction should be carried 
out with the hand in the position midway between flexion and extension, i.e. on a level with the forearm.

The action of the small muscles of the thumb is encouraged by gradually allowing the thumb to abduct by relaxing the adhesive plaster. It is a mistake to remove this completely until recovery takes place, as the powerful abductor longus

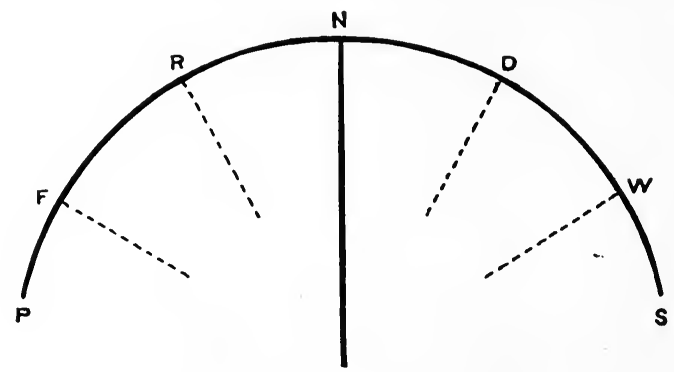

FIG. 5O.

pollicis (extensor ossis) and the extensor brevis will produce forcible abduction and extension of the thumb (fig. 46).

For re-education of the adductor the hand should rest on the table with the palm upwards, in the over-supinated position ; and for the flexor brevis in the position midway between pronation and supination.

The inner lumbricales are stimulated to work by encouraging flexion at the metacarpo-phalangeal joints of all the fingers-the interphalangeal joints being maintained in the position of extension by means of small straight finger splints. Until recovery is complete flexion of the two inner metacarpo-phalangeal joints should be insisted upon, as only in this way is hyper-extension at these articulations due to action of the unopposed communes tendons avoided. This hyper-extension is unfortunately a common feature of ulnar paralysis.

The weakness of the interossei is shown in the inability to extend at the interphalangeal joints. In what is usually demonstrated as a typical case of ulnar paralysis the condition is a flexion at the proximal interphalangeal joints, and 
a hyper-extension at the metacarpo-phalangeal joints of the ring and little fingers. There is no doubt that even after total division of the nerve and subsequent suture, recovery of the interossei of the middle and index fingers-the power to extend at the interphalangeal joints-is more rapid than

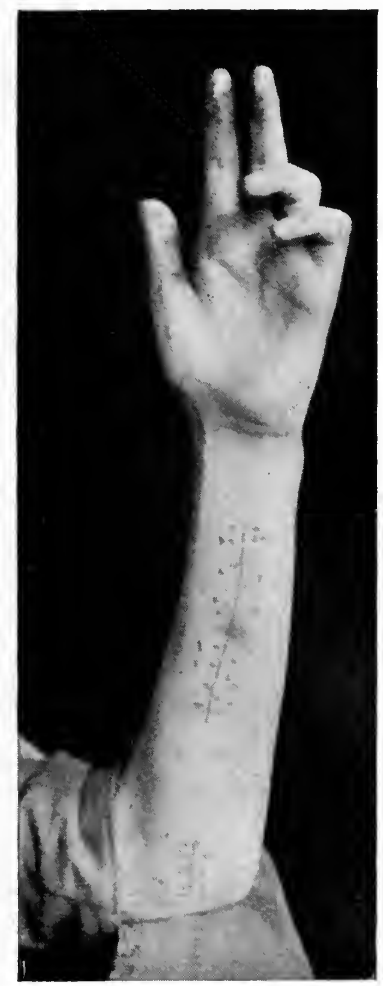

FIG. 5I.-Partial ulnar paralysis.

The interossei of the index and middle fingers have recovered. The interossei of ring and little fingets are still paralysed. There is inability to extend at the interphalangeal joints of these fingers (interossei); also inability to flex at the metacarpo-phalangeal joints owing to paralysis of the inner lumbricales. The fingers are flexed at the interphalangeal joints owing to action of the flexores profundis and sublimis.

in the case of the ring and little fingers (fig. 5I). One might have expected the reverse, since in these two fingers the opposition of the flexor profundus is present, and also the lumbriçales, whilst these are lost in the ring and little 
fingers. At first I was inclined to regard the good result in the case of the index finger as in some way associated with the extensor indicis, but careful dissections demonstrated that, like the communis tendon for the index finger, its motor effect did not extend beyond the proximal phalanx. The next explanation that suggested itself was: Could it be due to stimulated function on the part of accessoryif minute-branches of the median nerve to the outer interossei ? Of this I have no evidence in my notes carried over a period of twelve years in the dissecting room. Another explanation was that recovery of the interossei would manifest itself earliest and strongest in the fingers furctionally the most active. In the causation of this condition two other factors must be taken into consideration. With hyper-extension at the metacarpo-phalangeal joints of the ring and little fingers (over-action of communis and paralysis of lumbricales) there is an alteration in the line of pull of the interossei, which becomes angular instead of straight. Furthermore, an extended proximal phalanx would form a rigid dorsal support for the sublimis tendon, thus increasing its flexion pull.

Having as I thought exhausted all possible explanations an interesting phenomenon was noted four months after operation in a case of complete division of the median and ulnar nerves in the arm of a patient at the Military Orthopædic Hospital, Shepherd's Bush. The operation, which was performed by Lieutenant Teece, R.A.M.C., consisted in a repair of the ulnar nerve with a portion of the internal cutaneous and of the median with a portion of the great sciatic from an amputation stump. After the operation Lieutenant Teece kindly handed me over the case for observation and reeducation. We were dealing with a condition in which as regards all the fingers flexion power was lost as well as extension of the middle and distal phalanges. Recovery of power in the fingers was first obtained in the flexor sublimis, with ability to bend all the fingers at the first interphalangeal joint. This was median nerve recovery. As this flexor power increased it was noticed that there was 
comparatively easy extension at the interphalangeal joints (interossei) of the index and middle fingers, but in the ring and little fingers this was practically absent, owing to flexor contraction. It was clear that if this were not prevented the marked deformity of flexor contraction of the ring and little fingers characteristic of ulnar paralysis would be manifested. I may mention that the pronators were at this time acting strongly.

If all the interossei had a similar nerve supply there was no reason why the two inner interossei should have

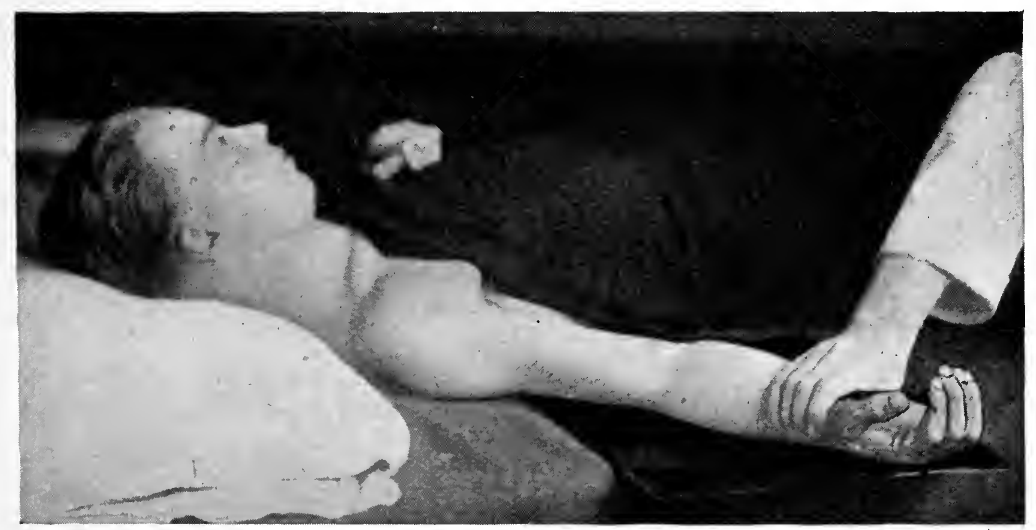

FIG. 52.-Testing and commencing re-education in weakness of the extensors of the fingers (interossei and extensor communis).

behaved differently from the two outer ones. There could to my mind be only one explanation, namely, that the median as well 'as the ulnar nerve was sending branches to the interossei of the index and middle fingers. These interossei, therefore, have a double nerve supply, of which the ulnar forms the principal portion, since though at the outset of ulnar paralysis we meet with weakness of the outer interossei this is not noticeable in cases of median paralysis. I have dealt rather particularly with this question because it is an important example of the value of functional anatomy as the great guiding factor in the display of any dissection, Another example may be also noticed 
in the occasional double supply of the profundus tendon to the middle finger. This is observed in some cases of median paralysis. In re-education of the interossei it is important not to take the fingers out of the splint unsupported, as the sublimis is always ready to flex acutely, and how can weakened interossei act against an unrestrained flexor sublimis? The fingers are best kept rested on an adjustable metal splint, and a little flexion allowed at the interphalangeal joints, so as to offer a minimum effort in extension. This is gradually increased as recovery takes

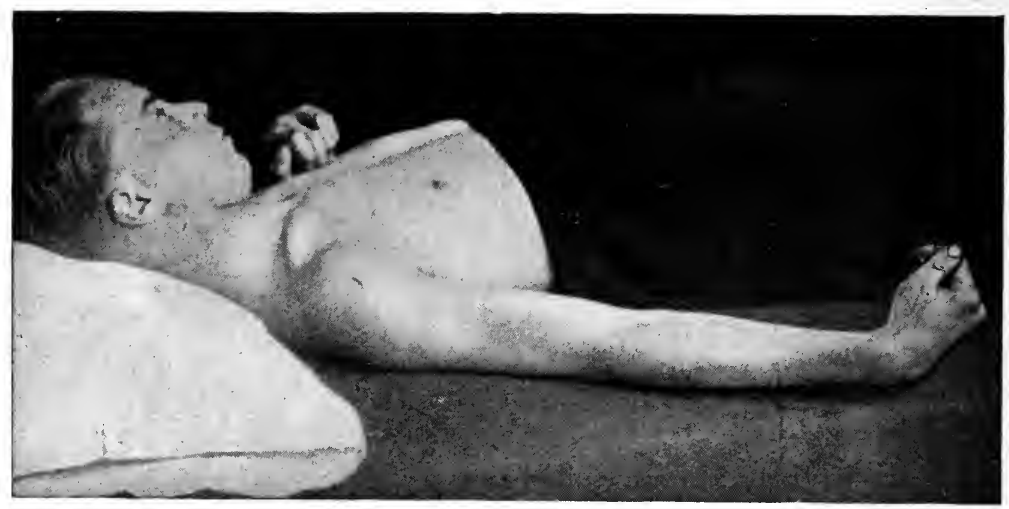

FIG. 53.-Increasing the work of the extensors of the fingers

place. The maximum recovery or Ioo units of work has taken place when the interossei can extend the two interphalangeal joints from acute flexion against gravity, the hand being over-pronated, the palm looking downwards and the dorsum upwards. Hence in commencing work for the interossei the hand should be at first over-supinated (fig. 52); by this means we obtain gravitational assistance. With recovery, just as in re-educating the wrist extensors, we gradually work to the mid prone and supine position (fig. 53), and finally to the position of over-pronation.

As regards weakened extension of fingers, whether of the interphalangeal joints (interossei) or metacarpo-phalangeal (ext. communis), it is important to remember, as previously 
stated, that this is aided by flexion of the wrist, whether the position of the forearm be that of pronation, or supination, or midway between the two. The position of extension of the wrist favours flexion of the fingers. Thus in testing for recovery of the interossei or extensor communis the wrist should be slightly flexed and the forearm placed in a position of over-supination. Increased work is given by gradually extending at the wrist to the position of complete extension. It is frequently found that movement of the

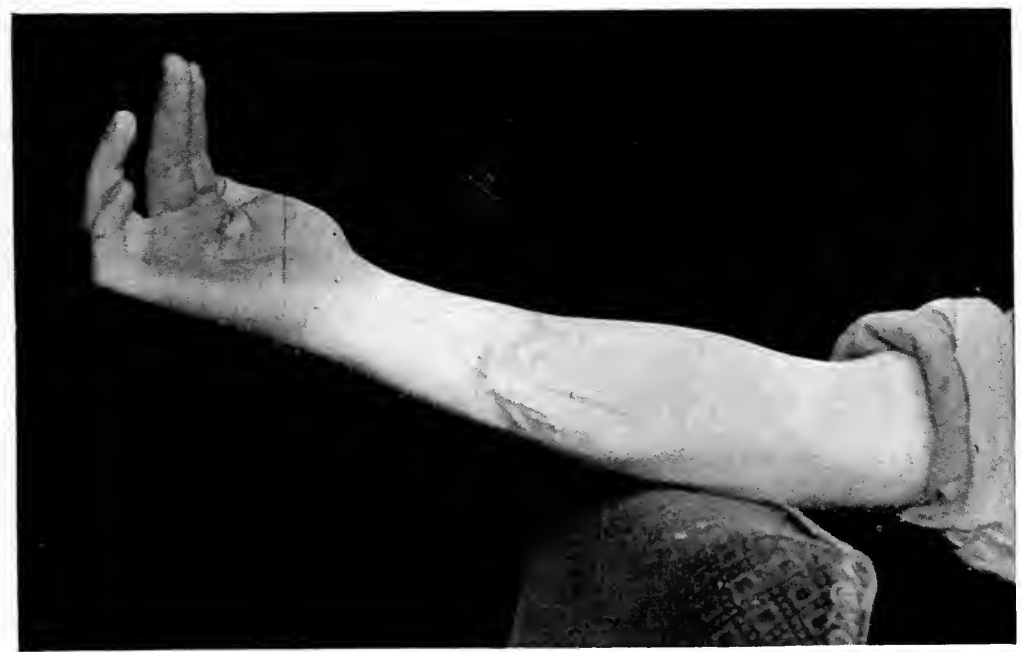

FIG. 54.-Partial ulnar paralysis.

The interossei of the index and middle fingers have recovered, and the two outer lumbricales are producing flexion of these fingers at the metacarpo-phalangeal articulations. The interossei and lumbricales of the ring and little fingers are paralysed. There is hyper-extension. at the metacarpo-phalangeal joints owing to action of the extensor communis from paralysis of the lumbricales. In spite of strong action of the flexor sublimis the patient is unable to bend the proximal phalanges.

interossei is aided by making pressure on the proximal phalanges of the patient.

In cases where hyper-extension at the metacarpo-phalangeal joint of the ring and little fingers has unfortunately (fig. 54) been allowed to occur, we find that the patient can extend at the interphalangeal joints when the metacarpophalangeal joints are flexed, but fails when they are extended. 
Hence when re-educating the interossei on the splint we should begin with flexion at the metacarpo-phalangeal joints, gradually extending them as recovery takes place.

It must be borne in mind that it is common enough to find sublimis contraction with flexion at the first inter phalangeal joints as a result of neglect to rest the interossei properly. I have never, on the other hand, seen any impairment to sublimis function follow when this treatment has been carried out. After the operation of suture for complete division of nerve there is no reason why movements should not be tested for and encouraged as soon as the surgical state of the wound is regarded as satisfactory. This would apply to the case of division of any nerve in the upper or lower limb. 


\section{CHAPTER VI}

\section{MUSCULO-SPIRAL PARALYSIS}

THE musculo-spiral nerve which supplies the extensors of the elbow, the wrist, thumb, and metacarpo-phalangeal joints of the fingers as well as the supinator brevis, may be regarded as a continuation of the posterior cord of the brachial plexus. It takes its rise from the fifth, sixth, seventh, and eighth cervical nerves. The order and position of origin of the muscular branches-a knowledge of which is important in cases of nerve injury-are as follows :

Triceps and anconeus (extensors of All branches to these are given off elbow)

$\{$ above lower third of the arm.

Brachio-radialis (accessory supinator (These muscles arise from the of forearm)

Extensor carpi radialis longus (extensor and radial abductor of hand at wrist joint) main trunk of the musculo-spiral immediately above the elbow joint. The remaining branches arise from the posterior interosseous division.

Extensor carpi radialis brevis (extensor and radial Immediately below abductor of hand at wrist joint)

Supinator brevis (supinator of forearm) the elbow, just before the posterior interosseous nerve enters the supinator brevis.

Extensor communis digitorum (extender of metacarpo-phalangeal joints of fingers)

Extensor minimi digiti (accessory extender of metacarpo-phalangeal joint of little finger)

Extensor carpi ulnaris (extender and ulnar abductor of hand at wrist joint)

In the upper third $\mathrm{Cl}$ forearm, immediate. below the supinator brevis. 
Extensor ossis metacarpi pollicis (long abductor of thumb) or abductor longus pollicis

Extensor brevis pollicis (extender of the metacarpophalangeal joint of thumb)

Extensor longus pollicis (extender of the interphalangeal joint of thumb)

Extensor indicis (accessory extender of metacarpophalangeal joint of index finger)

In the middle of the forearm.

It is important to note that the extensors of the wrist, extensor carpi radialis longus, extensor carpi radialis brevis, and extensor carpi ulnaris, all arise above the elbow joint, being traced to the lateral or external condyle-the longus, the highest in origin of all, arising from the supra-condyloid ridge.

The extensor communis digitorum and the extensor min. (quinti) digiti-really a portion of it -also arise above the elbow joint from the outer condyle of the humerus. The three extensors of the thumb, namely, extensor ossis or long abductor which is inserted on the radial side of the base of the first metacarpal, the extensor brevis pollicis which is inserted into the dorsal aspect of the base of the first phalanx, and the extensor longus pollicis which is inserted into the dorsal aspect of the base of the second or distal phalanx, together with the extensor indicis-constituting the deep layer of the muscles of the forearm-all arise below the elbow-joint. No branches are distributed from the musculo-spiral nerve to the small or intrinsic muscles of the hand. They receive their supply from the median and ulnar nerves.

The triceps, the antagonist of which is the brachialis, is not often affected in paralysis. It is a powerful muscle, and its strength is shown by the projection of the olecranon, into which its tendon is inserted.

Just as the biceps is the most powerful supinator in the flexed position of the forearm, so is the supinator brevis the most powerful supinator in the extended position. This interesting distinction can be made in cases of musculospiral affection in the arm, or in cases of musculo-cutaneous paralysis. 
The short, fleshy, and powerful supinator brevis is traced above to the lateral condyle of the humerus, and, passing from behind forwards round the outer side of the upper portion of the radius, is inserted into the front and outer aspects of that bone as low down as the junction of the middle and upper thirds.

The specialization of thumb movement in the human hand is well shown in the fact that the metacarpus, first phalanx, and second phalanx have each a separate extend-

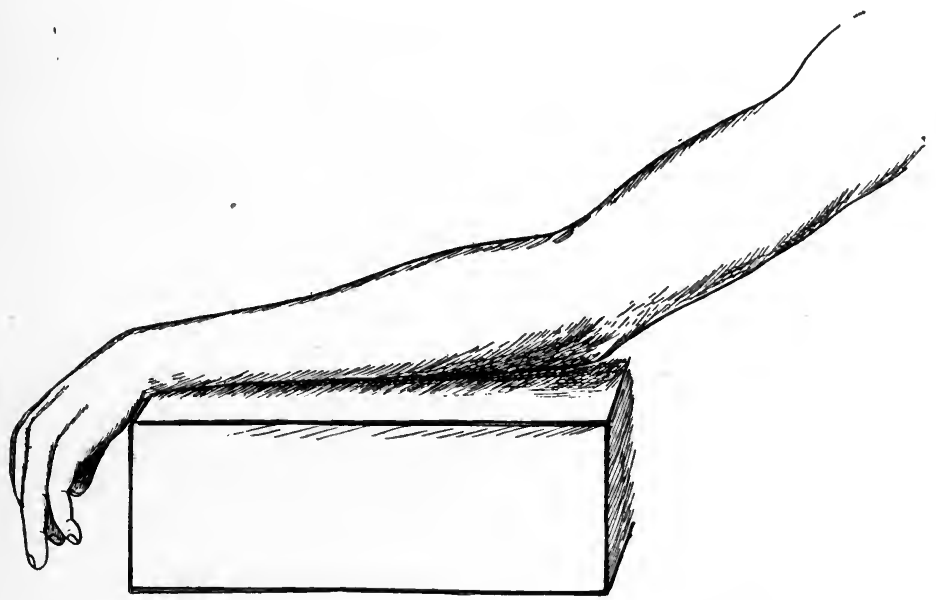

FIG. 55.-Musculo-spiral paralysis (Charles Bell).

Note the over-pronated forearm with elbow extension, wrist flexion, thumb flexed and adducted, flexion of metacarpo-phalangeal joints of the fingers (lumbricales). Extension can still be produced at the interphalangeal joints by the interossei, as is shown in the case of the middle finger.

ing muscle. In cases of thumb drop with paralysis of all three, should tendon transplantation be decided on it would be better to graft on to the extensor ossis (abductor) in preference to either the long or short extenders. From the point of view of utility, paralysis of the three extenders is not so severe as the loss of the power of opposition of thumb to fingers due to paralysis of the opponens pollicis.

With reference to the abduction power of the three extensors of the wrist, the extensor carpi ulnaris and extensors carpi radialis longus and brevis, it would appear that 
abduction, either to the radial side or to the ulnar, can only take place when the extensors and flexors of the wrist are in a state of physiological equilibrium as regards flexion and extension. Thus we can abduct readily to the ulnar side by the action of the flexor and extensor carpi ulnaris when the forearm and hand are on a level, and similarly to the radial side by the action of extensor carpi radialis longus and brevis and the flexor carpi radialis. If we acutely flex the wrist (palmar flexion), in which case the flexors are in a physiological state of contraction and the extensors physiologically relaxed; or acutely extend (dorsal flexion), in which case the extensors are contracted and the flexors physiologically relaxed, we are unable to perform this movement. The effort to perform this is then made by pronation and supination of the forearm and abduction and adduction of the fingers. The practical importance of this is in connection with re-education for either ulnar or radial deviation of the hand. This should be carried out with the hand midway between flexion and extension, on a horizontal level with the forearm.

Extensor Communis Digitorum.-Much obscurity surrounds the action of this muscle, for which there would appear to be little justification. It arises above the elbow from the outer condyle, has a thick fleshy belly, and at the lower part of the dorsal aspect of the forearm divides into three, sometimes four, tendons. These tendons diverge across the back of the hand, and, if only three exist, the inner one divides into two, one for the ring and one for the little finger. The tendon for the ring finger is connected to that for the little and middle fingers, but not to the first finger, by accessory bands or vincula, so that while extension of the ring finger is associated with that of the other two, there is free mobility of the index finger. On the dorsum of each proximal phalanx each tendon is usually described as forming an aponeurotic expansion, which is reinforced by the tendons of the lumbricales and interossei - that of the index finger being reinforced by the extensor indicis and of the fifth finger by the extensor minimi 
(quinti) digiti. The expansion then divides into three portions, a central inserted into the middle phalanx, and two lateral ones which unite and are inserted into the

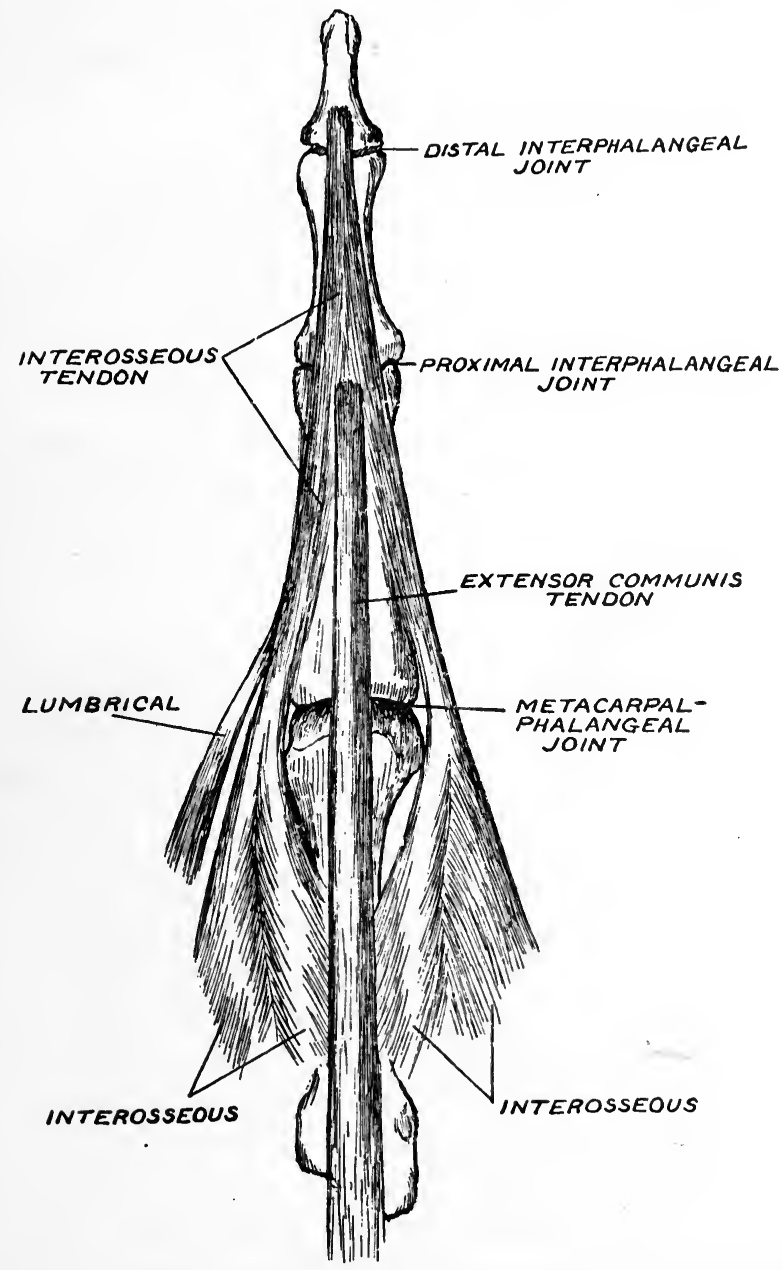

Fig. 56.-The arrangement of motor forces on the dorsum of the finger.

distal phalanx. Thus, from this description the communis when it contracted would act on all the phalanges producing extension, and being assisted in the case of the middle and 
distal phalanges by the interossei and lumbricales. That this description of function is erroneous can be demonstrated by two simple tests.

(a) If we hyper-extend at the metacarpo-phalangeal joints we can at the same time acutely flex at the interphalangeal joints. The hyper-extension at the metacarpo-phalangeal joints is due to the fact that the extensor communis is in a physiological state of contraction. If this muscle extended the middle and distal phalanges also, then, to allow acute flexion of the interphalangeal joints by the profundus and sublimis, the communis must be in a state of physiological relaxation and subsequent elongation. But, as has been explained above, a muscle cannot be in two physiological states, contraction and relaxation, at one and the same time.

(b) If we flex at the metacarpo-phalangeal joints the communis, which extends these, must be in a physiological state of relaxation with subsequent elongation, yet with these joints flexed we can fully extend at the interphalangeal joints. The extensor communis produces extension at the metacarpo-phalangeal joints. It does not produce extension at the interphalangeal joints.

If we examine the dorsum of a proximal phalanx, for example of the ring finger (fig. 56), the extensor tendon does not spread out into an aponeurosis. On the contrary, it is well defined, and is traced mesially to the distal part of the phalanx, where it is attached. The motor effect of contraction of the communis terminates there. Laterally we see the two well-defined tendinous edges of the interossei receiving on the radial side, just distal to the metacarpophalangeal joint, the insertion of the lumbrical-and these are traced on to the distal phalanx. On the dorsum of the proximal phalanx these lateral tendons are connected proximately to the communis tendon by thinner, and more distally by thicker strands, and also to each other on the dorsum of the middle phalanx. They finally converge, to be attached to the proximal portion of the terminal phalanx. If we carefully examine a dissection of the dorsum of the hand and fingers in which the joints are lax, fully extend 



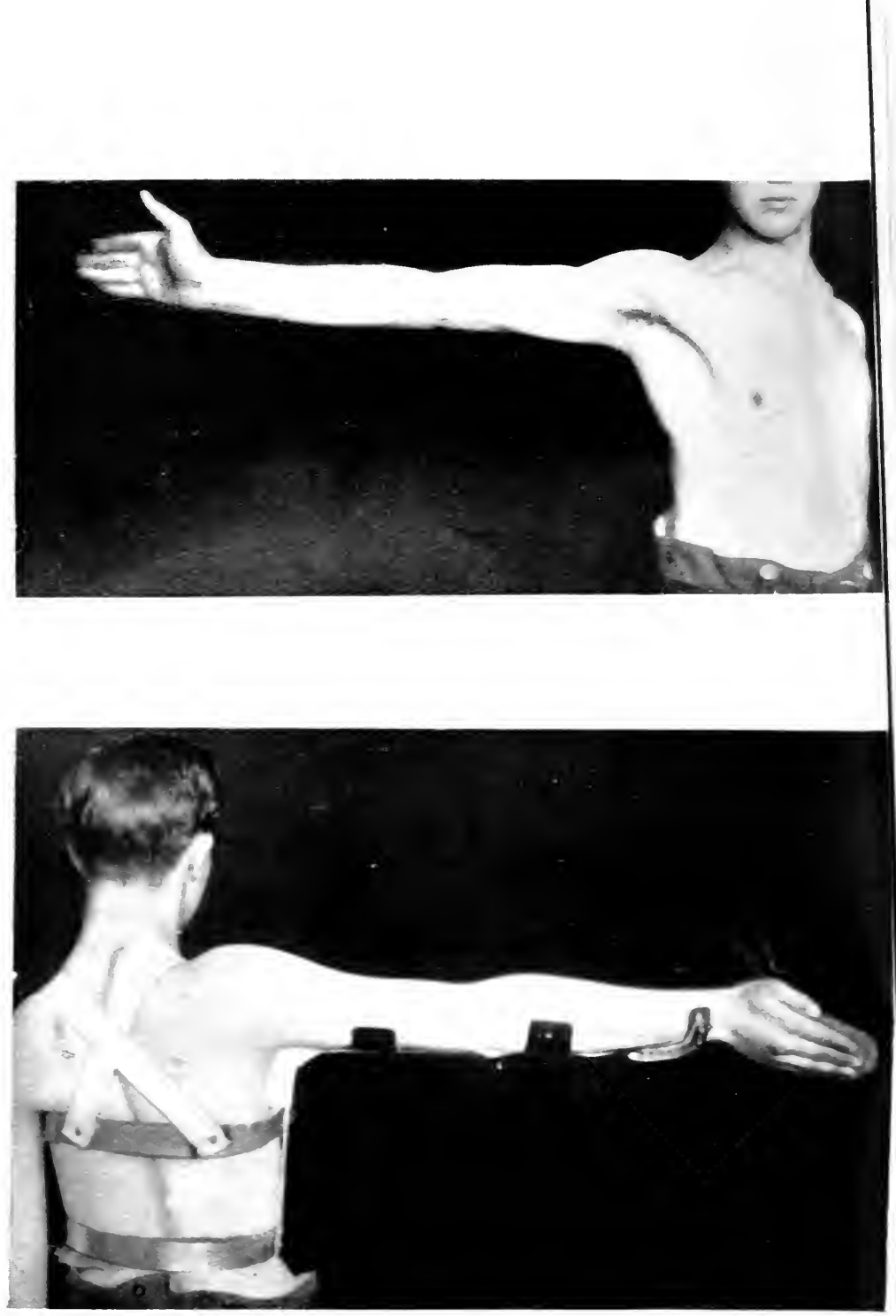

FIG. 5\%.-Position for immediate rest in musculo-spiral paralysis. 
the fingers and then, still keeping the metacarpo-phalangeal joint over-extended, flex at the interphalangeal joints, the result is to elongate, not the communis tendon, but the tendons laterally from the interossei. If we bend the metacarpo-phalangeal joint we immediately elongate the communis tendons.

\section{Anatomical Rest in Musculo-spiral Paralysis}

(I) The triceps not only arises by two heads, the lateral ind medial, from the outer border and dorsal surface of the humerus, but also by the long or scapula head which is traced to the rough triangular surface of the scapula immediately below the glenoid fossa. Hence, to rest most effectively our triceps it is not only necessary to extend the elbow joint so as to elongate the opposing brachialis, but also to elevate the arm so as to take the strain of the weight of the limb off the long head.

(2) The forearm is placed in the mid position between pronation and supination, or, better, in the slightly supinated position, and the wrist is hyper-extended (dorsi-flexed) so as to take all strain off the supinator brevis and the extensors of the wrist. In this way we antagonize the actions of the flexor carpi ulnaris, flexor carpi radialis, and pronators. Full supination in this position could not be borne by the patient.

(3) The thumb is extended and held forcibly abducted by means of adhesive plaster. Too much attention cannot be paid to adequate rest of the long abductor of the thumbthe extensor ossis metacarpi pollicis.

(4) The fingers are best extended at the outset, not only at the metacarpo-phalangeal articulations, but at the interphalangeal joints as well. In this way the lumbricales, the opponents of the extensor communis, are relaxed and elongated. Although communis power terminates at the proximal phalanx, nevertheless, as a result of the connection of their tendons on the dorsum of this phalanx with the interossei tendons, adequate rest is not secured unless the interossei are rested as well, i.e. unless the interphalangeal 
joints are extended. Only in this way is the greatest resistance offered to the most effective factor in preventing recovery in a case of musculo-spiral paralysis, namely active contraction of the flexors of the fingers and wrist. The greater size of the medial or inner condyle of the humerus gives us an indication of the relative power of the two groups. These positions can be effected by means of the abduction upper limb splint, with the hand-piece dorsi-flexed and slightly supinated. They are admittedly not positions of comfort, but after an operation on the musculo-spiral nerve or at the onset of paralysis they are those which give most complete rest. Furthermore, in severe old flexor contractions (which it may be stated should never occur), these are the positions in which the greatest tension and stretching force is placed on the tightened tendons. In the absence of triceps affection and when the flexion contraction is not severe a " long cock-up " metal or aluminium splint may be used. This is bent at the wrist to give the necessary dorsiflexion, and on it the front of the forearm and hand rest.

In the absence of contracture of the flexors, and with commencing recovery of the common extensor this splint need only extend to the distal portion of the proximal phalanges, leaving the middle and distal phalanges free. In cases where the extensor tendons of the wrist only are affected, the " cock-up" splint may only extend from the middle of the forearm to the metacarpo-phalangeal joints. Numerous patterns of these " cock-up" splints

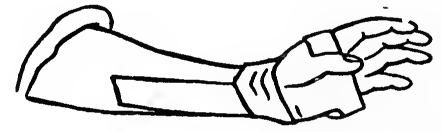

FIG. 58.-Resting the hand in paralysis of the wrist extenders (Thomas).

are in use, and instead of being made of metal they may be made of plaster moulded to whatever position in which it is desired to place the hand. All are modelled, however, on the original " drop wrist " metal splint (fig. 58) designed many years ago by Mr. H. O. Thomas. 
Method of Re-education in Musculo-spiral Paralysis

It is presumed at the outset that in a case of nerve injury or division surgical repair has been effected. The treatment of this form of paralysis is practically the treatment of "dropped wrist," in which we include the thumb and the fingers at the metacarpo-phalangeal joints. The basic principles for the treatment of this condition were first enunciated by $\mathrm{H}$. O. Thomas. Instead of assuming that the condition of wrist drop was permanent and irremediable, Thomas argued that the primary cause might have been temporary in its effects; and that elongated but recovered extensors were mechanically prevented from acting owing to the contraction of their opponents. The extensors were not of necessity permanently paralysed and stretched.

For purposes of prognosis Thomas used the following test : "If the patient be asked to try and perform by the exercise of the will only greater flexion than already exists, the fingers flex readily, and also by the act of the will extend forward again to the position of previous repose; furthermore, while the wrist is firmly flexed by supplemental assistance, the patient can by the exercise of his will within a small radius rapidly flex and extend his fingers." In treatment he placed the hands and fingers by means of the long cock-up splint in the position of extreme extension, " so as to allow the muscular tissue of the extensors of the wrist and fingers to retract from the overstrain or overdraw." On removal of the splint by the surgeon to test the result; " should the patient by the exercise of his will be able to maintain extension the surgeon may allow the limb to be used, discontinuing the angular extension apparatus." Thomas recognized that a muscle, though weakened in action or " paralysed," might have recovered; but it was prevented from acting simply because during its period of weakness its opponent had shortened, with the result that it itself was held mechanically elongated. He did not of course regard this simple treatment as universally successful in all cases of dropped wrist. In cases of division of the musculo-spiral 
nerve and subsequent repair, or in cases of poliomyelitis, rest in the dorsi-flexed wrist position does not of itself effect recovery, though in the latter, if the case is seen early, this may be all that is necessary.

If we place the patient's wrist and hand in the position of extension at the outset, we have made a good beginning on the road to recovery. No attempt at re-education should be commenced till we are assured that we have overcome "flexor" contracture. Weakened muscles must not be asked to commence work at a mechanical disability. The hundred units of work, or maximum recovery, will have been attained when the patient can not only extend at the wrist and metacarpo-phalangeal joints from acute flexion with the dorsum of the hand uppermost, i.e. in the position of over-pronation; but can also similarly abduct the thumb with extended phalanges.

For exercising, the patient should lie on the back with the head supported by a pillow, and the arm with the elbow slightly flexed should rest on a firm pillow placed parallel to the patient's side. A sheet of cardboard should be placed under the limb. When the hand is taken off the splint it should be placed in the position of over-supination

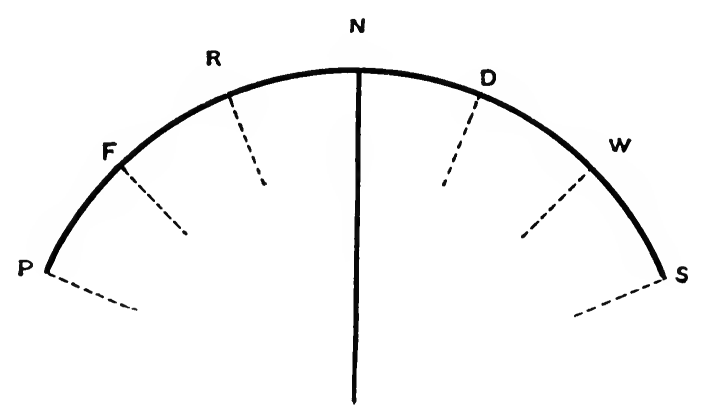

FIG. 59

(S, fig. 59), the palm of the hand being uppermost. The thumb, while the wrist and fingers are being exercised, should be held abducted by a strip of adhesive plaster. In the supinated position slight flexion of the wrist and fingers is permitted and the patient asked to extend. In 


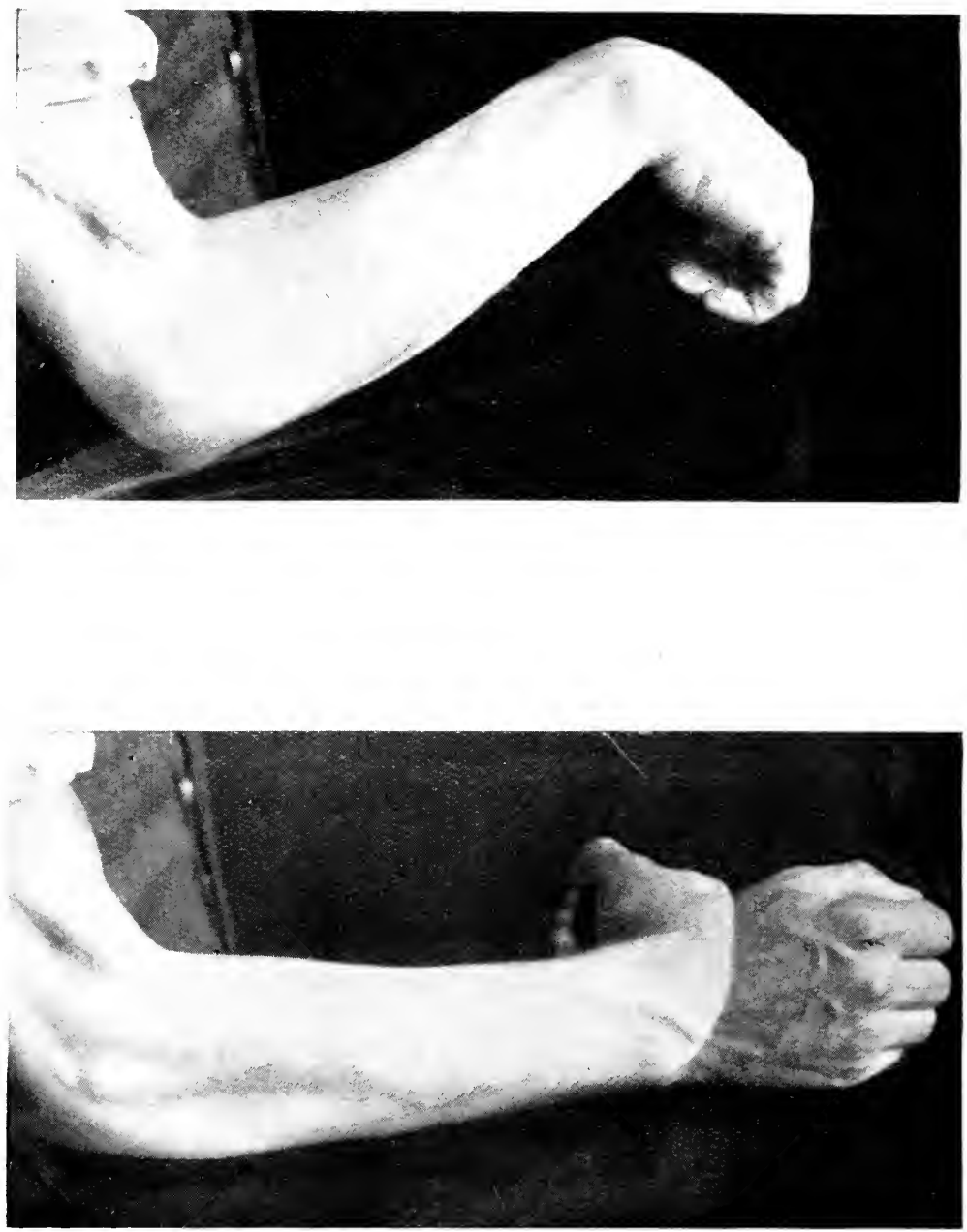

FIG. 60.-Musculo-spiral paralysis.

The patient is unable to extend the wrist in the over-pronated position, but can do this when the hand is placed midway between pronation and supination: 
this position we obtain the benefit of gravitational effect, and with flexion at the wrist and metacarpo-phalangeal joints leverage action of the weakened extensors is improved, since at these joints artificial pulleys are formed. This is similar to the effect of extension of the wrist in helping the action of weak long and short flexors of the fingers. The flexion is gradually increased till finally the patient can extend from full flexion. This would constitute recovery in this position. The amount of flexion can be graduated by means of an aluminium "cock-up" splint. Help may be given to the tendons by grasping the forearm of the patient above the wrist. As regards the thumb, slight adduction is at first allowed by slacking the adhesive plaster and the patient is encouraged to abduct. Similarly slight flexion (gradually increased) is permitted at the metacarpophalangeal and interphalangeal joints to encourage extension, and the latter may be assisted by compression of the proximal phalanx and of the metacarpus. In some cases it may be found advisable to prevent flexion at the interphalangeal joints of the fingers by means of small straight splints, only allowing movement at the metacarpo-phalangeal joints.

As regards extension of weakened fingers, whether of the interphalangeal joints (interossei) or metacarpo-phalangeal (extensor communis), it is important to remember that this is aided by flexion of the wrist, whether the position of the forearm be that of pronation, or supination, or midway between the two. The position of extension of the wrist favours flexion of the fingers. Thus, in testing for recovery of the extensor communis or interossei the wrist should be slightly flexed, and the forearm placed in a position of oversupination. Increased work. is given by gradually further extending at the wrist.

The hand of the patient is allowed gradually to approach from $\mathrm{W}$ and $\mathrm{D}$ to the mid prone and supine position $\mathrm{N}$ (fig. 59). Thus at $\mathrm{W}$ a similar procedure to the above is adopted, and recovery obtained before pronating to $\mathrm{D}$, and similarly at $\mathrm{D}$ before pronating to $\mathrm{N}$. The arc is gradually increased 
to $\mathrm{R}$ and $\mathrm{F}$, till finally the over-pronated position $\mathrm{P}$ is reached, the patient can then extend both wrist and digits with the dorsum of the hand uppermost (fig. 6o). If the patient cannot extend the wrist in the mid prone and supine position $\mathrm{N}$, it is positively certain he will be unable to do it in the over-pronated position $P$. In cases of musculo-spiral paralysis much patience and continuous work may be necessary to obtain recovery. When it is decided to commence re-education, twenty minutes at least

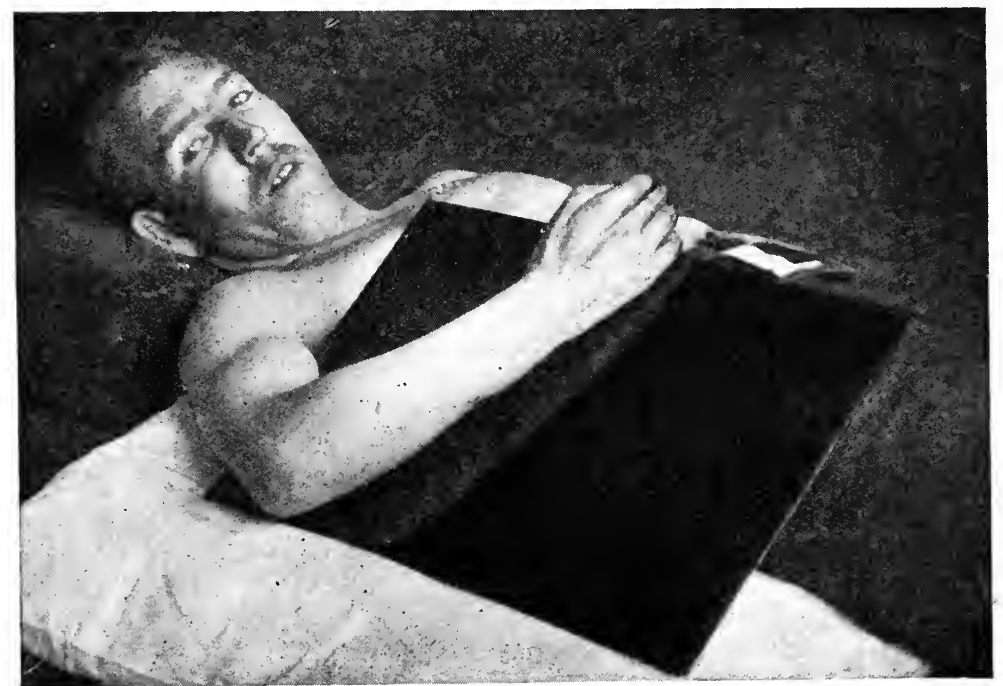

FIG. 6I.-Re-education of the triceps.

four times a day should be devoted by the attendant to this procedure.

Supinator Brevis.-In cases where this muscle is affected, this would be manifested by difficulty in supinating in the extended elbow position as compared with that in the position of flexed elbow. With the elbow extended and the patient lying down we would work gradually along the arc S P (fig. 59), being certain we could supinate along the arc $\mathrm{D} S$ before we attempted from $\mathrm{N}$, the mid prone and supine position. We have reached our Ioo units of 
work or maximum effort when we can supinate from $\mathrm{P}$, the position of over-pronation.

Triceps.-The patient should be lying down and the limb placed on a pillow and cardboard as above described (fig. 6I). A C B (fig. 62) represents the elbow extended, and D C the forearm at a right angle. With the elbow flexed to E 45 degrees we ask the patient to extend along the arc E B. Then along the arc D B and then F B. Recovery takes place in this position when the patient extends the elbow

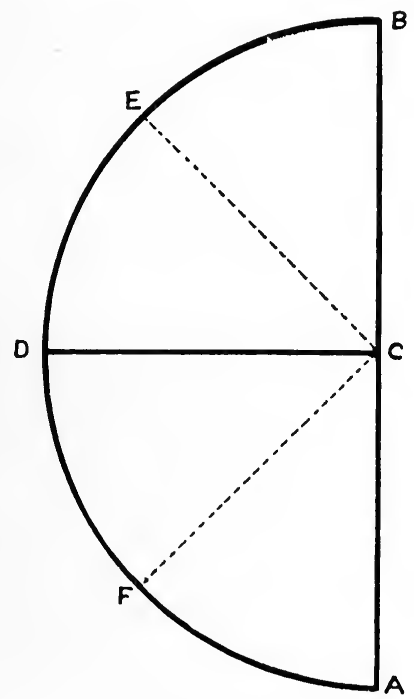

FIG. 62.

from the position of acute flexion. Further work is given to the triceps by removing the pillow, and with the patient still recumbent and the arm resting on the table the arc of flexion is again gradually increased till the patient extends from full flexion. Also with the elbow and arm raised, first on a small and later on a large pillow, and the forearm and hand dependent towards the patient's body, the triceps is asked to extend the forearm against gravity. This is the reverse method of re-education to that described for the 
brachialis. The patient is then gradually elevated by means of pillows to the erect position-the same positions and methods being repeated-recovery is complete when the patient, sitting or standing up, can extend the elbow from full flexion, controlling it at any angle of extension.

Division of the Lumbricales.-As the lumbricales are the physiological antagonists of the communis muscle the question may arise whether division of these is not justifiable in old cases with flexor contraction which are not responding to treatment. As the profundus and sublimis tendons are intact and would permit of the coarser flexion movements, the loss of the lumbricales would be of little moment compared with the recovery of the dropped fingers. The tendons of the lumbricales can be picked up where they join the tendinous expansions from the interossei, about $\mathrm{I}^{\cdot} 5 \mathrm{~cm}$. above the metacarpo-phalangeal joints in an adult (fig. 56, p. II5). A vertical incision is made on the middle of the lateral or radial surface of the proximal phalanx of each finger.

The Movements of The Thumb and Fingers in Median, Ulnar, AND Musculo-spiral Paralysis

Median Nerve.-(I) Inability:

(a) Cannot flex the second phalanges of any fingers (flexor sublimis).

(b) Cannot flex the third or distal phalanges of the index and middle fingers (flexor profundus).

(c) Cannot flex the proximal phalanges of the index and middle fingers (two outer lumbricales).

(d) Cannot flex the thumb (flexor longus and flexor brevis pollicis), nor oppose the thumb to the fingers (opponens pollicis).

(2) Ability:

(a) Can abduct or adduct the fingers (interossei).

(b) Can extend all the fingers at all the joints (interossei and extensor communis).

(c) Can bend the ring and little fingers at the metacarpo- 
phalangeal articulation (two inner lumbricales) and at the distal interphalangeal joints flexor profundus.

(d) Can extend and abduct the thumb (extensor brevis and extensor longus pollicis and the long abductor or extensor ossis).

(e) Can adduct the thumb (adductor pollicis).

Ulnar Nerve.-(I) Inability:

(a) Cannot flex the proximal phalanges (lumbricales) or distal phalanges (flexor profundus) of the ring and little fingers.

(b) Cannot abduct or adduct the fingers (interossei).

(c) Cannot extend the middle and distal phalanges of any fingers (interossei).

(d) Cannot adduct the thumb (adductor pollicis).

(2) Ability:

(a) Can flex all the phalanges of the index and middle fingers (flexor sublimis, flexor profundus, and lumbricales).

(b) Can flex the middle phalanges of the ring and little fingers (flexor sublimis).

(c) Can extend the proximal phalanges of all the fingers (extensor communis).

(d) Can oppose the thumb to the fingers and also flex and extend as well as abduct it (flexores longus and brevis pollicis, extensores longus and brevis pollicis, long and short abductor pollicis, and opponens pollicis).

Musculo-Spiral Nerve.-(I) Inability:

(a) Cannot extend the phalanges or metacarpus of the thumb (extensor longus, extensor brevis, and extensor ossis or long abductor).

(b) Cannot extend the proximal phalanges of any fingers (extensor communis).

(2) Ability:

(a) Can flex, oppose, and adduct the thumb (long and short flexors, opponens, and adductor pollicis).

(b) Can abduct and adduct the fingers (interossei).

(c) Can flex the proximal phalanges of all the fingers (lumbricales).

(d) Can flex and extend the middle and distal phalanges 
of all the fingers (flexor sublimis, flexor profundus, and interossei).

In considering the movement of abduction of the thumb, I regard the extensor ossis or long abductor (musculo-spiral nerve) as the important factor, rather than the short abductor pollicis (median nerve). 


\section{CHAPTER VII}

\section{THE MUSCLES OF THE THIGH}

THE movements of the thigh bone or femur, all of which are performed at the hip joint, are :

(I) Flexion.

(2) Extension.

(3) Adduction or movement inwards towards the opposite thigh.

(4) Abduction or movement outwards.

(5) External rotation.

(6) Internal rotation.

The muscles performing these movements arise from the pelvis, except the psoas which arises from the lumbar spine. They are all examples of the third order of levers, in which the power lies between the weight to be moved and the centre of motion at the hip joint or fulcrum.

These movements are performed by the following muscles :

Flexion

Iliacus, psoas major

Adduction

Adductor magnus

Adductor longus

Adductor brevis

Pectineus

Rotation outwards

Pyriformis

Gemelli

Obturator internus

Obturator externus

Quadratus femoris
Extension

Gluteus maximus

Abduction

Gluteus medius and Tensor fasciæ femoris

Rotation inwards

Gluteus minimus 
There are two principal regions where these muscles are inserted, namely the great trochanter and the lesser trochanter. The insertions of the adductors are however traced down the shaft of the femur along the linea aspera. When we speak of thigh movements we presume of course that the thigh is not fixed by the body weight; in other

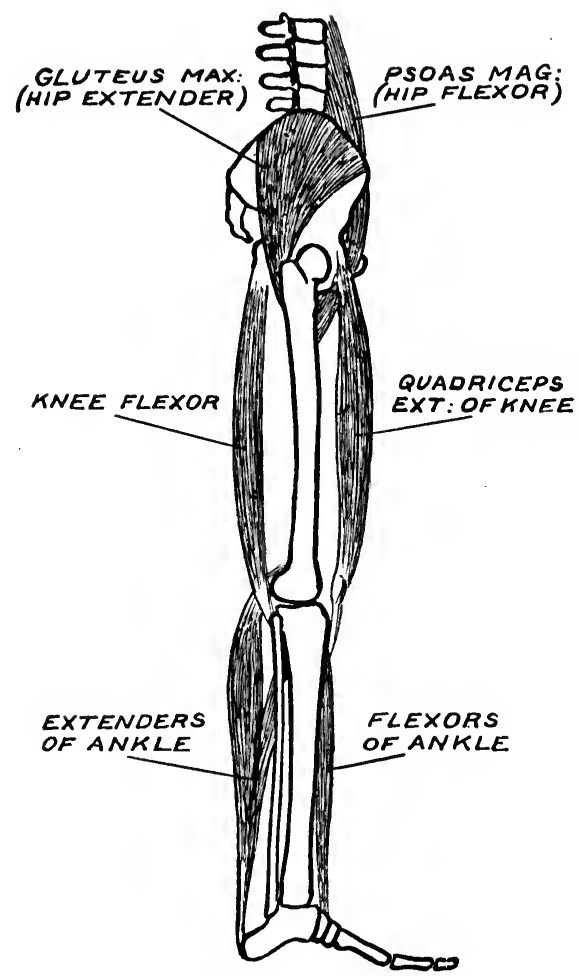

FIG. 63.-The right lower limb supporting the trunk in the erect position owing to contraction of the gluteus maximus and the quadriceps.

words, we are not using it for support ; or, rather, that it is not fixed by muscle so as to support the weight of the body. The muscles which act on the free thigh, with the trunk of the body fixed, also act on the free trunk when the thigh is fixed. Thus, according to the free or fixed condition of the thigh do we designate the origin and insertion of the 
muscles. If we stand on the right lower extremity (fig. 63), which we are enabled to do by extending the knee (quadriceps) and the hip joint (gluteus maximus), we can readily flex the left thigh (ilio-psoas) on the trunk and extend it readily also (gluteus maximus). . If we stand on both lower limbs, the knees being extended (quadriceps), we can bend the trunk on the thighs at the hip joint

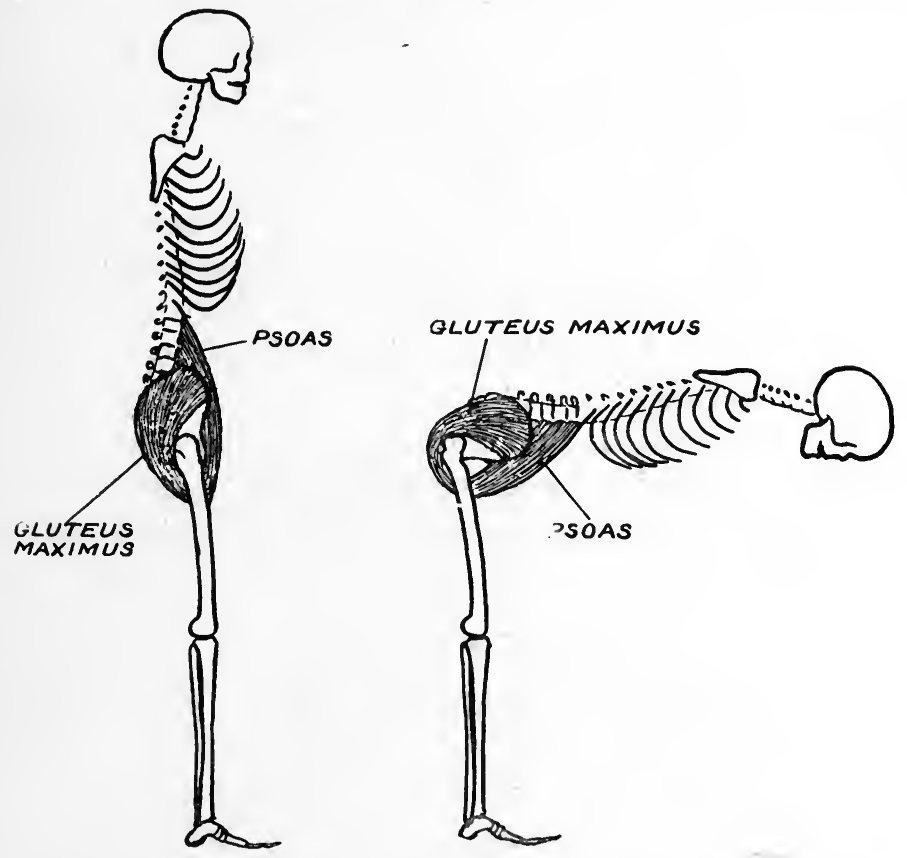

FIG. 64 .--Flexion and extension of the trunk on the thigh.

(ilio-psoas), as in stooping to pick up an object from off the ground, and extend it to resume the erect position (gluteus maximus). The lower limbs then become fixed points on which the trunk flexes and extends (fig. 64). Although the knee and ankle, like the hip, bear the whole weight of the trunk, yet no joint in the body is the subject of more continuous movement than the hip; apart from the fact that in comparison with the knee and ankle it allows movement in all possible directions. 
When we stand on our lower limbs, although the body weight is transmitted unfavourably, the share taken by each femur is equal, and the centre of gravity falls exactly in the middle between the two thighs. But if we abduct the trunk on to one or the other limb the body weight is not transmitted to the ground through the neck, or weakest part of the femur, but through the middle of the bone, which is its strongest part. The obliquity and length of the neck of the femur, though predisposing to fracture and so constituting a source of weakness, nevertheless, are essential, since it is necessary that the femur should move freely in all directions.

The lower limbs of man are relatively longer and larger than those of any other member of the mammalian order. The prominences of the femur are more marked, the neck more oblique, and the pelvis is wider. These, together with the great development of the buttock and back muscles, especially the gluteus maximus, are related to man's erect posture, and admit of freedom as supporting agents of the fore limbs, so that they can respond readily to the requirements of the will.

\section{Flexion AND Extension}

Flexion movement at the hip joint, whether of the thigh on the trunk or of the trunk on the thigh, is performed by the iliacus and psoas magnus muscles. The psoas is a long and powerful fleshy muscle arising within the abdomen on each side from the lumbar vertebræ. It is traced ventral to the sacro-iliac articulation, and coming into relation with the iliacus passes with it beneath Poupart's (inguinal) ligament, and is inserted by a tendon into the lesser trochanter of the femur. The iliacus, also a strong fleshy muscle, arises within the pelvis from the iliac fossa and base of the sacrum. It is traced with the psoas beneath Poupart's ligament to be inserted partly into the tendon of the psoas, partly into the lesser trochanter, and partly into the femur-for about I inch-immediately below the trochanter. The two muscles being so closely related and having a similar 



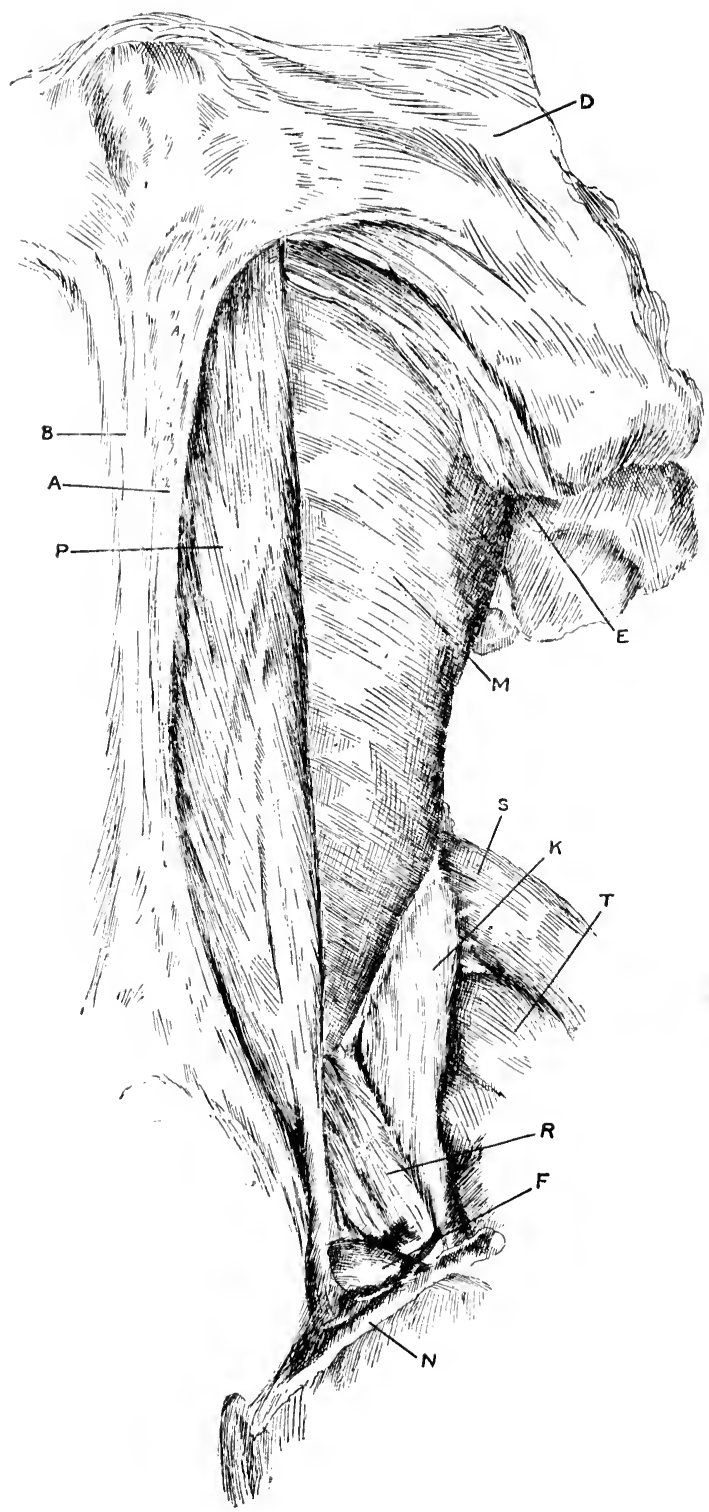

FIG. 65.-The dorsal abdominal wall of the kangaroo.

D, Diaphragm. E, Last dorsal nerve. A, Aorta. B, Post caval vein. P, Psoas minor. M, Quadratus lumborum. R, Psoas major. K, Iliacus. N, Epi-pubic bone. F, Pectineus. S, Sartorius. T, Rectus femoris. 
insertion are designated ilio-psoas. In addition to the power of flexion of the hip outward rotatory powers have been ascribed to the ilio-psoas. It is a pure flexor, however, as we can bend the thigh in the position of either internal or external rotation. If the ilio-psoas were an external rotator, then to allow internal rotation it would have to be in a state of relaxation and bending of the thigh should then be impossible. In connection with the development of the erect attitude as exemplified in man the four principal muscular changes to be noted are :

(I) Deltoid development.

(2) Selection and enlargement of the psoas major and the diminution in size, or complete disappearance of the psoas minor.

(3) The great relative size of the gluteus maximus.

(4) Functional, not structural, increases in the quadriceps.

In the case of the kangaroo, representing an experiment towards the erect position at an early stage of mammalian development, we find that the psoas minor is the great psoas and that the muscle corresponding to the psoas major of man is the smaller. The psoas minor on each side forms in the kangaroo a large fleshy mass (fig. 65) traceable from the lower dorsal and lumbar regions to the tubercle of the pubis. In this marsupial the " erect posture" is not formed by a trunk resting on two practically vertical stems. The erect position is maintained by means of a long foot and an enormous tail propping up the trunk behind-the knee and hip joints being flexed. It is an erect position in the sitting posture-the tail in the kangaroo corresponding to a chair as used by man (fig. 66). In the platypus also, representing in various directions the lowest form of mammalian life, the psoas minor is a relatively large muscle in relation with the thoracic and lumbar vertebræ, while the psoas major is comparatively insignificant. The erect position in man has necessitated powerful muscles for support about the centre of motion of the body, which is at the hip joint. 
The development of powerful extension function (gluteus maximus) has called into being powerful flexion. The psoas, instead of being a pelvic muscle, has as it were incorporated into itself the belly of the psoas minor, and has become an abdominal muscle, thus increasing its effectiveness as a bender. There is no muscle in the body whose action is of more diagnostic significance than that of the psoas magnus of man. Flexion of the thigh, due to reflex contraction of the ilio-psoas with relaxation and elongation

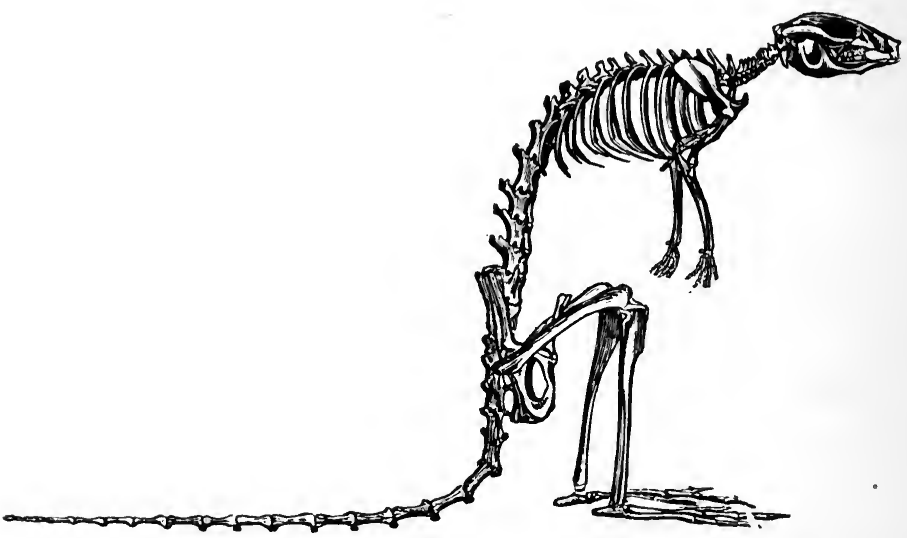

FIG. 66.-The erect posture of the kangaroo.

of the gluteus maximus, is associated with many pathological conditions, such as the passage of a stone in the ureter, appendicitis, and inflamed lymphatic glands in children, etc.

The great value, however, of psoas contraction as a diagnostic help is in connection with disease of the lumbar vertebræ and joints, sacro-iliac joint, and the hip joint, with all of which it is in relation. In hip joint disease however, limitation to elongation of the psoas is associated with limitation of movement in all directions. The psoas, by its-contraction, acts as a sentry and places the hip joint in the position of greatest relative ease. If we forcibly extend the hip the shortened psoas arches the spine, producing lordosis; and if we flex the hip we straighten the spine. H. O. Thomas, recognizing this 
factor, rested the joint, by means of his dorsal hip splint, in the flexed position assumed by the joint for the purpose of decreasing tension-the deformity was then gradually reduced and the angle of the splint altered as the joint recovered. The psoas was gradually allowed to relax and elongate, and the gluteus maximus to contract and shorten. The method of forcible reduction, as by weight and pulley, is antagonistic to the principle that regards psoas contraction as nature's means of placing the joint in a state of physiological rest.

It was John Bell's opinion, over a century ago, that the pernicious doctrine of forcibly resisting the contractions of the muscles gave rise to those torturing machines for the
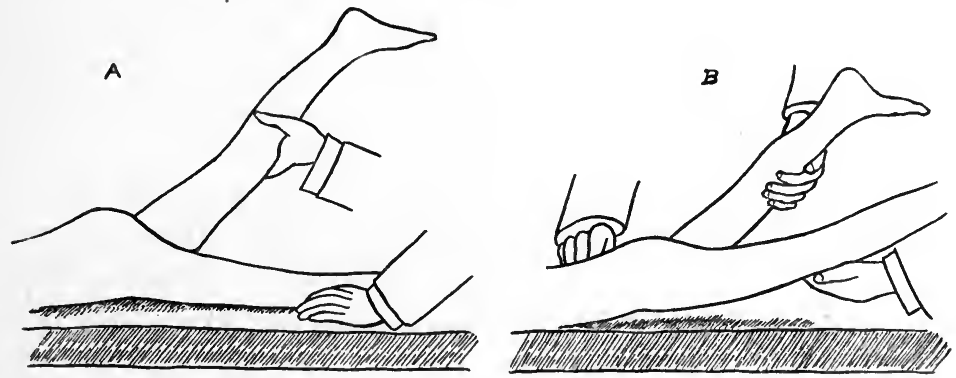

FIG. 67.-Testing for psoas irritation.

treatment of conditions about the hip which were associated with the names of Gooch and Wathen. If we place a healthy child in the prone position and grasp the extended knee of either side the amount of extension at the hip joint, or in other words, of relaxation and elongation of the psoas, is sufficient to allow the thigh to be brought almost to a right angle to the pelvis (fig. 67A). In cases of suspected psoas irritation this forms a very delicate test. On the normal side there is this free extension, on the abnormal the patient will limit the extensor action of the gluteus maximus, which necessitates relaxation and elongation of an irritated psoas, and will simulate extension by raising the pelvis on the affected side (fig. 67 в). 
This is a serviceable test in cases of suspected psoas abscess. Opposing the ilio-psoas we have the powerful fleshy Gluteus Maximus. This arises from the upper and back part of the dorsum ilii, back of the sacrum and coccyx, and from the great sacro-sciatic ligament. The fibres pass obliquely downwards and outwards, and are inserted by a somewhat broad and flat tendon into the fascia lata and for about $7 \mathrm{~cm}$. along the gluteal ridge, between the great trochanter and the linea aspera on the posterior aspect of the femur (fig. 72, p. I43). This muscle is the extender at the hip joint. With the pelvis fixed and the hip flexed it extends the femur so as to bring the thigh in a line with the body. Further extension (dorsi-flexion) is however permitted, as is seen particularly in children and in dancers who, with the body supported on one lower limb, are able to touch the back of the head with the other heel, the action of the gluteus maximus being assisted by flexion at the knee joint. After we stoop to pick up an object the gluteus maximus, using the femur as a fixed point, draws the pelvis backwards, and with it the trunk, thus enabling the body to assume the erect posture. Its action is seen also when we rise from the sitting to the erect posture. When we sit with the body erect we rest the feet on the ground and the knees and hips are flexed. When we rise, we extend at the hip by the gluteus maximus, and at the knee by the extensor quadriceps. The gluteus maximus is also described as an abductor and external rotator, but this is not so. We can extend at the hip in either the positions of adduction or of internal rotation; and if the gluteus maximus were an abductor or outward rotator, then, during adduction or internal rotation, it would be relaxed, and so could not contract to extend. The relatively great size of the gluteus maximus is essentially a characteristic of man and a necessity of the erect position. Various experiments have been made by nature with this muscle at different stages of mammalian development. In the platypus (fig. 68) the fibres of its large fleshy belly converge behind the leg between the biceps cruris and spur, and twisting 
on themselves are inserted at the middle of the tibia below the inner hamstrings. It acts as a bender of the knee. In the kangaroo the insertion has retracted to the thigh and

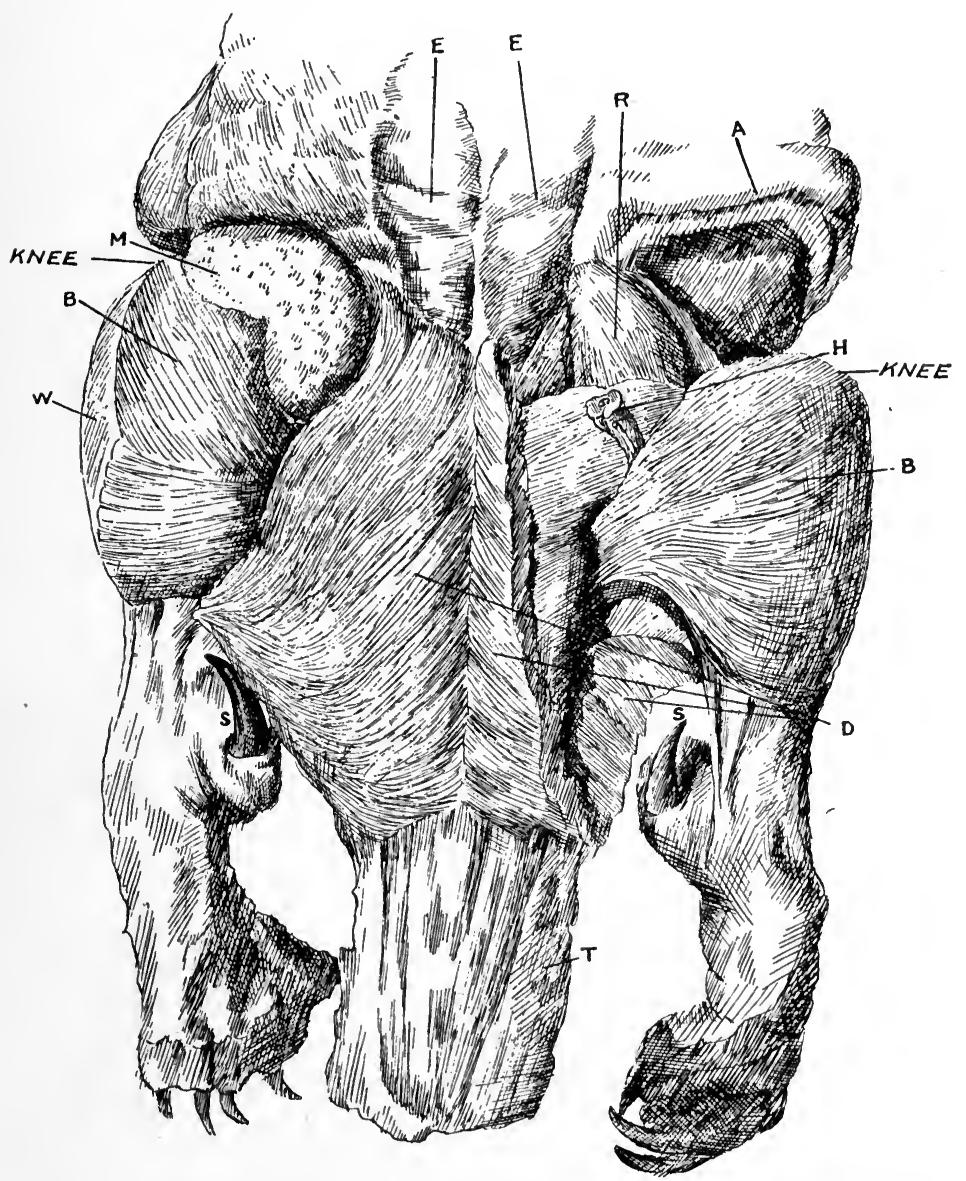

Fig. 68.-Dorsal dissection of platypus to show gluteus maximus and biceps cruris.

s, Spurs, in which duct of gland terminates. M, Poison gland. $\mathbf{H}$, Part of gland and duct passing behind biceps. E, Erector spinæ. R, Meso-gluteus. A, Abdominal muscles. r, Part of tail. D, Ecto-gluteus (maximus). W, Anterior tibial muscle. B, Biceps femoris.

its narrow tendon is traced along the lower third of the femur. Though it acts in this marsupial as a hip extender 
it does not present the relative predominance in size over the meso-gluteus which we meet with in man (fig. 69). Even in the anthropoid apes the development of the outer gluteus is small compared with that of man, for whilst according to Macalister it forms I9\% of the musculature round the hip in man, it forms $13 \%$ in the gorilla, $12.5 \%$ in the chimpanzee, and only $\mathrm{I} 2 \%$ in the orang. The femur in the anthropoid is always at an angle with the trunk. It never approaches the vertical as seen in man. In the shuffling gait of the chimpanzee seen in any menagerie, the ape prefers to balance himself by the use of the long fore limbs in preference to depending solely on the hip and knee muscles. Of all the anthropoids the gorilla in its attitude most nearly approaches the erect posture of man.

\section{AdDuction AND AbDuction}

The three adductors, which were described by the older anatomists under the name triceps femoris, represent a strong group of muscles running from the pelvis (arising from the outer surfaces of the pubis and ischium anterior to the centre of the hip joint) downwards and outwards to the inner side of the thigh. They are inserted along the linea aspera on the back of the femur and extend from the lesser trochanter to the inner condyle.

The Adductor Longus arises from the os pubis at the junction of the crest with the symphysis by a narrow tendon, which in a case of division is exposed one inch below the bone, and is the most superficial of the three adductors.

The Adductor Brevis, which lies behind the longus, arises farther out from the mid line, from the body and descending ramus of the pubis.

The Adductor Magnus, the largest of the three, arises still more dorsally from the rami of the pubis and ischium, as well as from the tuberosity of the ischium.

The Longus passes downwards, outwards, and backwards, forming a well-defined fleshy belly ending in a flat tendon, which is inserted into the middle of the linea aspera on the inner lip for about 4 inches. 


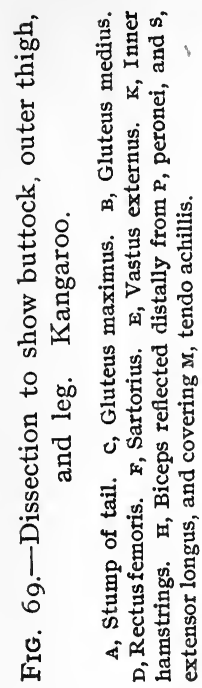


The Brevis, like the longus, has a narrow origin, but expands into a fleshy belly and is inserted by a flat tendon into the linea aspera behind and above the longus, and reaching as high almost as the lesser trochanter. Its fibres run less obliquely than the longus, since, owing to the

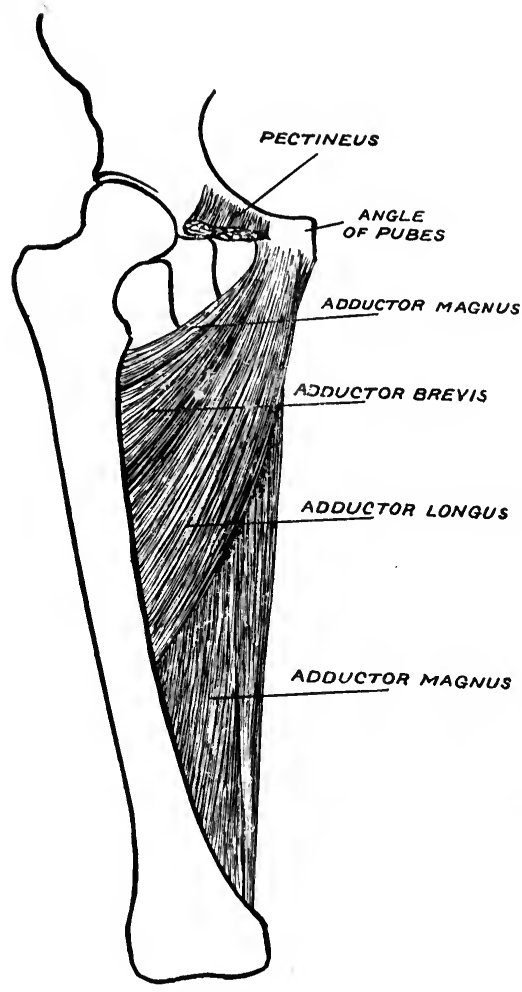

FIG. 70.-The adductors of the thigh.

position of its insertion and its size, it passes more directly between the pelvis and the thigh.

The Magnus, which lies behind the longus and brevis, is inserted along the whole length of the linea aspera, extending below to the adductor tubercle on the internal or medial condyle. The upper fibres pass almost directly across to the upper part of the linea aspera from the pelvis, while the lower pass obliquely downwards and backwards, 
and the muscle forms what is practically a partition between the front and back of the thigh.

Pectineus.-This arises from the ilio-pectineal line and the adjacent outer surface of the os pubis, and passes outwards, downwards, and backwards, to be inserted at a rough line leading from the lesser trochanter to the linea aspera.

Both the adductor longus and the magnus are inserted at a distance from the centre of motion at the hip joint, but compared with the other muscles acting on the femur the pull of the adductors is more at a right angle than oblique. This increases their power. These muscles, when the pelvis is fixed, all adduct the femur, bringing it inwards towards the mid line, i.e. towards the opposite thigh. In riding these muscles bring the knees of the horseman against the animal's flanks. With the thighs fixed they are important muscles in maintaining the equilibrium of the erect posture, preventing the trunk being abducted to one or the other side. In standing erect we have our thighs moderately abducted, the base between the anterior extremity of each foot being about twelve inches. That would represent the state of equilibrium between the abductors and adductors. If we stand on the right foot only, that necessarily means an abduction of the trunk on the right thigh, since the body weight is transferred through that femur to the ground. The adductores of that side have relaxed and the abductor, the gluteus medius, has contracted. These states can be increased by throwing the trunk more to the right side. As the body weight is taken off the left limb the movements on the left side are abduction or adduction of the thigh on the trunk according to will.

External rotatory and other powers have been also ascribed to the adductores. Their action is solely to draw the thigh inwards. This can be done with the thigh in the position either of flexion or extension or of inward or outward rotation. If these muscles were outward rotators also, then, if we internally rotated the thigh, they should be in a physiological state of relaxation. 
Yet in this state they can also contract, since we can adduct readily the abducted and internally rotated thigh. In spastic diseases seen in children, spasmodic contraction of the adductors forms a great barrier to walking, the knees approach each other and even cross, and there is no base or interval between the feet necessary for the maintenance of the erect posture. Whether the primary trouble is associated with the contracted adductors, or with the controlling antagonistic abductor is difficult to state. In standing erect it is essential for equilibrium that the vertical from the centre of gravity, which is situated at the spinal column about the lumbo-sacral region, should fall within the base of support, i.e. between the two feet, which in ordinary standing are usually separated anteriorly by about 12 inches. With adduction of the thigh the feet are approximated to one another and the supporting base lessened, with the result that the slightest movement to one side or the other will throw the vertical outside of the base. Various measures have been adopted to overcome this when it is permanent, such as removing a wedge from the adductors, etc. As a rule division of the tendon of origin of the adductor longus and of the middle of the fleshy

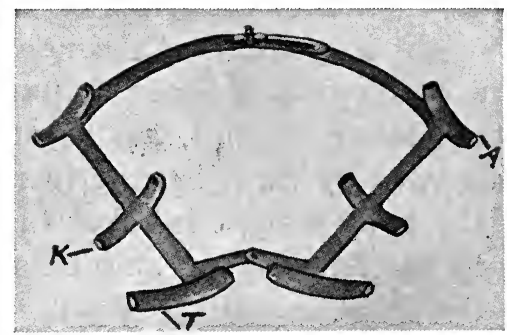

FIG. 7I.-A simple abduction or adduction frame.

$\mathrm{T}$, Upper thigh wing. $\mathrm{K}$, Knee wing. A, Ankle wing.

gracilis, which is associated with flexion of the knee, is sufficient in the absence of feet deformities - the patient being placed with the thigh forcibly abducted on the simple abduction frame shown in fig. $7 \mathrm{I}$. It is worthy of note 
that in a dissection of the ventral surface of the thigh in the platypus the gracilis is seen to be a broad fleshy muscle covering the adductores, semi-tendinosus, and semi-membranosus.

Abduction.-The antagonist to the adductores is the Gluteus Medius which abducts or draws the thigh outwards when the pelvis is fixed, or abducts the trunk when the thigh is fixed. In walking, when we stand on our right limb so as to allow the left limb to make a step, we abduct the trunk on the right thigh so that the weight of the body is transmitted through the right femur to the ground. This fan-shaped broad and fleshy muscle, the posterior third of which is covered by the gluteus maximus, arises from the outer surface of the pelvis, from that portion of the outer surface of the ilium between the upper curved line and iliac crest above, and the middle curved line below. The fibres converge to the great trochanter, where they are inserted by a strong flat tendon into the post-superior angle and the outer surface (fig. 72). The length of the tendinous insertion of the gluteus medius is about 3 inches. If we look at the hip joint from the outer side, the insertion of this muscle is immediately above the trochanter, and shortening of its fibres must result in abduction of the thigh. It is also stated by some authorities to have powers of extension and internal rotation, but we can abduct in the position of either flexion or of external rotation of the hip. If the medius were an extender or internal rotator it could not contract so as to abduct, since in these positions it would be relaxed. Compared with the adductores the gluteus medius has its insertion only a short distance from the centre of motion in the hip joint. Though having a short pull this is direct, and, furthermore, it has a powerful muscular belly.

Assisting the gluteus medius in its action as an abductor is the Tensor Fasciæ Femoris. This takes its tendinous origin from the fore part of the crested ilium for about $\mathrm{I}_{2} \frac{1}{2}$ inches. It passes downwards and obliquely backwards, and becoming thick and fleshy about the middle is inserted 
into the fascia lata on the outer side of the thigh at about the junction of the upper and middle thirds. As this muscle is inserted not into the bone, but into the fascia enveloping the muscles of the thigh, its use, from the direction of the fibres and position of origin and insertion, is to pull the thigh outwards from the mid line.

\section{External AND INTERnal Rotation}

The muscles which rotate the thigh outwards on the trunk, or the trunk outwards on the thigh, are six in number, and, coming from behind, they are inserted mainly on the inner surface of the great trochanter after bending round the axis of the femur.

The Pyriformis is one of the direct muscular connections between the axial skeleton and the lower limb. It arises from within the pelvis from the front of the sacrum, emerges by the great sacro-sciatic foramen, and is inserted into a facet on the upper and inner aspects of the great trochanter.

The Obturator Internus with the Superior and Inferior Gemellus can, from the point of view of insertion, be regarded as one muscle. The three muscles end in a common tendon which is inserted to a facet on the inner surface of the great trochanter close to the obturator externus and below the pyriformis. The superior gemellus arises from the spine of the ischium and the inferior gemellus from the ischial tuberosity. The obturator internus arises from within the pelvis, however, from the inner surface of the outer and front wall of the pelvis around the inner side of the obturator foramen. It leaves the pelvis through the lesser sacrosciatic foramen, the margin of which is grooved, cartilaginous, and lined with a synovial bursa, thus forming a pulley round which the tendon plays, by which means the leverage is improved.

The Obturator Externus.-This triangular muscle covers the outer or lateral surface of the front wall of the pelvis. It arises from the margins of the obturator or thyroid foramen formed by the pubic bone and ischium, and from 
the outer surface of the obturator membrane, and passing backwards and out on the dorsal aspect of the neck of the femur is inserted by a strong tendon into the digital fossa at the roof of the great trochanter. It is a short muscle, and is broad at its origin but narrow at its insertion, and twists round the thigh bone between it and the pelvis.

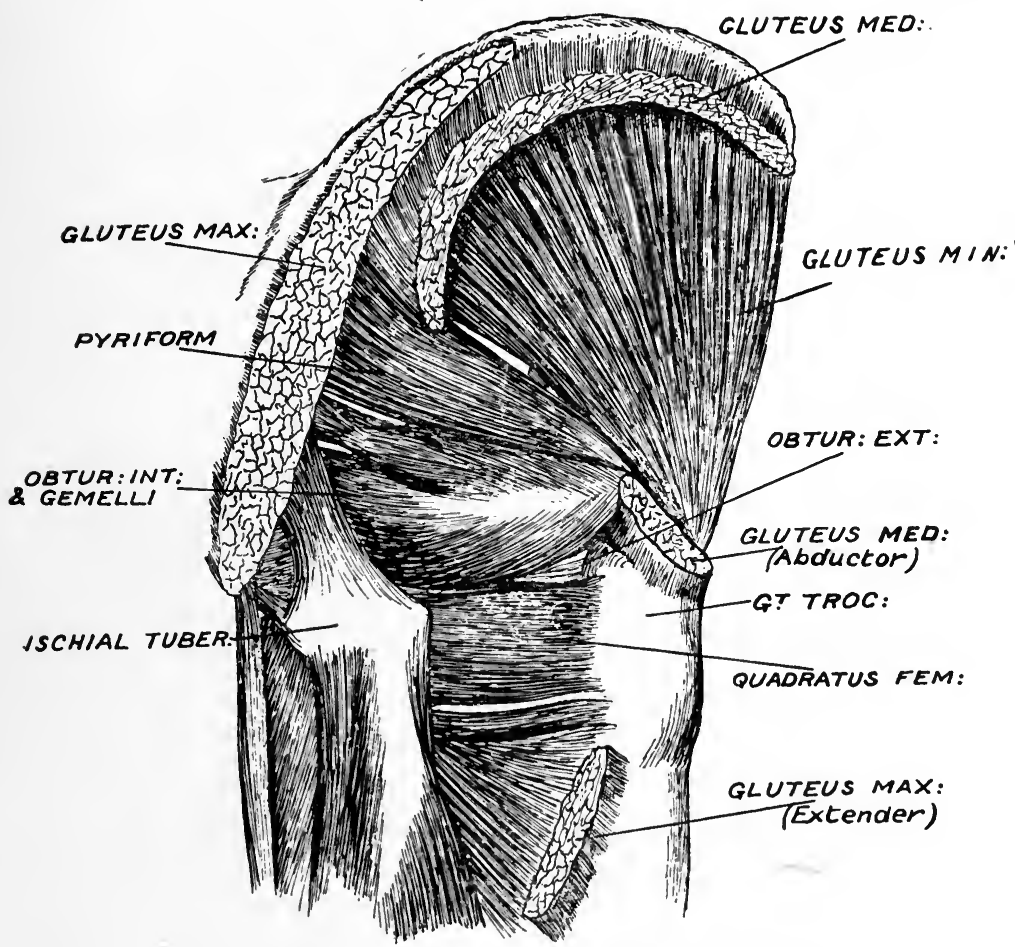

FIG. 72.-The muscles which rotate the femur.

The Quadratus Femoris is a thin muscle measuring about one inch from its upper to its lower border. It passes from the outer border of the tuberosity of the ischium to be inserted into the back of the femur between the two tuberosities below the level of the inter-trochanteric line. It is interesting to note that, whilst we depend on a single muscle for inward rotation, a series of six are necessary for outward 
rotation. By this multiplicity of muscles, flexion and extension are unimpaired, and by this provision accommodation is found for the numerous muscular insertions which are bunched round the trochanters so as to allow movement at the hip joint in every possible direction.

Internal Rotation is performed by theGluteus Minimus, which is the physiological antagonist of the six external rotators. It arises from the outer surface of the ilium, dorsal to the gluteus medius, between the middle and lower curved lines, and in front extends almost as far forward as the crest of the ilium, and behind reaches to the front of the great sciatic notch. It is inserted into the anterior surface of the great trochanter (fig. 72). The length of the insertion is about $I$ inch and width about $\frac{1}{2}$ inch. The tendon of insertion, which is about I' 5 inches long, describes a curve round the front of the great trochanter and lies more or less parallel with the neck of the femur, so that owing to the direction of its pull internal rotation will be produced.

Rotatory movements are the most limited of all the motions taking place at the hip articulation. In standing, for example, on the left lower limb with the right free, if we keep the right knee extended we can rotate the right thigh inwards or outwards on the fixed trunk with ease. As we flex the thigh this becomes more difficult, owing to the obliquity of the axis of the trunk with the femur, and in the position of acute flexion it becomes almost impossible. This applies similarly to the action of the trunk on the thigh. When we sit on a chair with the knees and hips flexed, rotatory movements of the femur are practically impossible, owing to the angle formed by the axis of the trunk with the thigh. The opposing or reciprocal muscles of rotation can be regarded as being in a state of equilibrium with each other. Nor can we roll the trunk on the femur. Even if we raise the right foot off the ground we cannot rotate the thigh on the trunk, but if we extend the right knee, which is associated with diminished hip flexion, then rotation can be effected; and this is especially so if we lean back on the chair so as to render the thigh and trunk level. 


\section{CHAPTER VIII \\ MUSCLES ACTING ON THE LEG}

THE muscles which move the leg are divided into two groups, benders and extenders. The centre for their motion or fulcrum is at the knee joint, and they are all examples of levers of the third order, in which the power is inserted between the part to be moved and the fulcrum. In all cases the insertion of the power is close to the fulcrum.

The flexors are six in number, five having an inner or medial insertion and one an outer or lateral insertion.

The inner flexors are:
Sartorius.
Gracilis.
Semi-tendinosus.
Semi-membranosus.
Popliteus.

The outer one is:

\section{Biceps cruris.}

The popliteus, though part of its insertion is seen on the inner side of the tibia, passes across the back of the joint from the outer to the inner side.

The extensors are four in number:

Rectus.

$\left.\begin{array}{l}\text { Vastus externus. } \\ \text { Vastus internus. } \\ \text { Crureus. }\end{array}\right\}$ Forming the Quadriceps Extensor. 


\section{FLEXORS}

Sartorius. - This is the longest muscle in the body and crosses the front of the thigh obliquely like a strap from the outer to the inner side. Its origin is from the pelvis in the region of the anterior superior spine, and it passes behind the inner condyle of the femur, to be inserted into the upper part of the inner surface of the shaft of the tibia. It was called the sartorius, or tailor's muscle, because it was supposed to abduct the thigh enabling one leg to be crossed over the other. This is erroneous.

The squatting position assumed by tailors depends on flexion of the knee and abduction and external rotation of the thigh.

Gracilis. - This is a fleshy narrow muscle placed superficially on the inner side of the thigh. It arises from the descending ramus of the pubis near the symphysis, and is inserted, like the sartorius, into the upper part of the inner surface of the shaft of the tibia below the tuberosity. In addition to its power as a flexor of the knee it has been described as an adductor of the tibia. Both the sartorius and gracilis bend the leg on the thigh when the latter is fixed. They arise from the front of the pelvis at either extremity of Poupart's (inguinal) ligament. The hamstrings arise at the back of the pelvis. The best demonstration of flexor action is seen when a person lies on the back and, with the knee extended and leg projecting over the end of a table, bends the extended knee. Also when the individual lies on the opposite side, for example the left, and with the right knee extended, resting against the left knee, acutely bends the right one. When the elbow is bent the forearm is directed to the front of the body; in the case of the knee the leg is directed towards the back. When, with the body lying altogether on a level surface, a bed or table, we flex the knee, we necessarily flex the thigh as well at the hip, otherwise movement could not occur. Flexion of the hip and knee are not, however, necessarily accompaniments of each other. They are independent 
movements. Thus the individual, when lying on the back in bed, can acutely flex the hip with the knee extended, and then flex the knee with ease.

The following functions are also ascribed to these muscles :

(I) By continuing to act they are said to flex the thigh on the pelvis.

(2) Taking their fixed point from below, i.e. with the lower extremities steadied, they fix the pelvis on the thigh, helping to maintain the body erect, and may further flex the pelvis on the femur.

(3) Powers of abduction and external rotation of the thigh are ascribed during flexion to the sartorius, and of adduction to the gracilis.

These muscles are not attached to the femur. They run between the pelvis and the leg, and therefore motion, the result of their action, takes place at the knee, not at the hip. The fulcrum or centre for action is not the hip joint, but the knee joint. They do not bend at the hip joint, and they can act as flexors of the knee with the thighs extended. Similarly they do not act as abductors, adductors, or thigh rotators, since they can act as flexors of the knee in the position of abduction, adduction, internal or external rotation of the hip. With the knee extended, rotatory powers at the hip joint might naturally be ascribed to these muscles; but in the act of extension at the knee by the quadriceps, these muscles would necessarily relax and elongate, and so could not contract and shorten in order to move the femur.

As regards the supposed power these muscles have when, by taking their fixed point from below, i.e. with fixation of the lower extremities, they help to maintain the erect position or even bend the trunk on the femur, we again have to remark that the fulcrum for their action is not at the hip but at the knee. These muscles do not bend the trunk at the hip, or act as supports in the erect position. Fixation of the lower extremities to allow these muscles to take their fixed point from below, so as to bend the trunk 
at the hip, necessitates knee extension. To allow the quadriceps to extend the knee, the gracilis and sartorius as flexors would of necessity be relaxed and elongated, and so could not contract to produce further action at the pelvis. In spastic paraplegia of children, in which adduction of the thigh is a marked feature, the gracilis is contracted and shortened as well as the adductors of the thigh. But its contraction is associated with accompanying knee flexion, of which it is the main cause, and not with adduction.

Semi-tendinosus. - This muscle was so named owing to the great length of its tendon of insertion, which usually begins about 5 inches above the knee joint. It arises, like the long head of the biceps cruris and the semi-membranosus, from the back of the tuberosity of the ischium. It lies on the inner and back of the thigh, and is inserted, like the sartorius and gracilis, into the upper part of the inner surface of the shaft of the tibia just below the inner tuberosity. These three insertions lie close to one another, being more or less united and almost implanted like one muscle tendon.

Semi-membranosus. - This lies, like thesemi-tendinosus, on the inner and back of the thigh, and like it also arises from the back of the tuberosity of the ischium. It received its name from the fact that it begins and ends in a flattened somewhat membranous-like tendon. It is inserted more proximal than the three preceding muscles, into the back of the inner tuberosity or condyle of the tibia above that of the popliteus.

Biceps Cruris.-This is the single bender inserted on the outside, and runs from the pelvis to below the knee. It has two heads of origin, a long and short. The long head arises, like the semi-tendinosus and semi-membranosus, from the back part of the tuberosity of the ischium. The short head arises from the outer lip of the linea aspera on the back of the shaft of the femur.

This short head begins about the junction of the upper and middle thirds of the femur. The common tendon of insertion is into the outer side of the head of the fibula, 
and by a fibrous extension to the outer tuberosity or condyle of the tibia, and sometimes into the outer side of the leg as well. The "hamstrings" is the term applied to the three flexors placed on the back of the thigh, namely, biceps, semi-tendinosus, and semi-membranosus.

Popliteus.-This muscle and the short head of the biceps are the only knee flexors not connected with the pelvis. It is a triangular muscle lying at the back of the knee joint, taking its origin from the outer aspect of the outer condyle of the femur and, crossing the joint, passes obliquely downwards and inwards, and is inserted into the dorsal surface of the upper part of the shaft of the tibia above the oblique line.

The Hamstrings, like the sartorius and gracilis, taking their fixed position at the pelvis, flex the knee. The popliteus, taking its origin from the fixed femur, also flexes the tibia. As to the action of the hamstrings when the fixed point is at the leg much confusion seems to have arisen. They have been described as taking a very important part in maintaining the body erect. For example, it is stated that "if we stand erect and bend the body forwards at the hip joint, on feeling the hamstrings it will be found that they are in strong action to prevent the trunk from falling forwards, and are the chief factors concerned in bringing the body back again to the erect position." When we stand erect our knees are extended owing to quadriceps contraction, which necessitates a state of relaxation and elongation in the opposing flexors.

When we bend the body forwards at the hip joint in the erect position, this is due to the action of the ilio-psoas taking its fixed point from the thigh, and is permitted owing to associated relaxation and elongation of its opponent the gluteus maximus (fix. 64, p. I29). The body is brought back to the erect position by the contraction of the gluteus maximus, and the relaxation and elongation of the iliopsoas. The hamstrings feel tight, not owing to the action of contraction and shortening, but to that of elongation. This is accentuated by the necessity, furthermore, of accommodating themselves during flexion of the 
trunk to the altered state of the hip joint over which they pass. Taking their fixed point below, their action is to bend the trunk and thigh on the leg at the knee joint, and this can be associated with either flexion or extension at the hip. Thus it is possible to bend the rigid trunk and thigh back at the knee joints, and though the ordinary individual can do this only slightly, acrobats and athletes can produce a marked flexion backwards. This is a pure action of the knee flexors-the quadriceps being relaxed and the condition of the hip being one of extension owing to the contraction of the gluteus maximus and the relaxation of the ilio-psoas-the remaining hip muscles being in a state of equilibrium.

In this backward action of the flexors we see the importance of the direct pull of the popliteus, for the reason of the retention of which in man I was often puzzled. As the extending vasti also arise from the femur and the rectus from the pelvis we have acting on the knee both on the front and back of the thigh, long and short levers. Taking their fixed point below, the most important action of the knee flexors is in connection with the apparently simple phenomenon of sitting. When we stand erect we have extension of the knee, the extensors being contracted and the flexors relaxed and elongated. When we sit on a chair there is flexion at the knee and hip joints, and the body is supported on the ischial tuberosities between which the vertical, through the centre of gravity, passes. In sitting down the legs and feet are fixed points, although it is interesting to note that when sitting, which means mechanical support, we can flex and extend the leg on the thigh. To acquire the erect position again we must have the legs and feet as fixed points, just as when we sat down. The flexion at the hip joint during sitting is a bending of the trunk on the femur by the ilio-psoas, the gluteus maximus being relaxed. The flexion at the knee is a flexion of the trunk and femur on the leg at the knee joint, owing to contraction of the flexors of the knee, and relaxation and elongation of the quadriceps extensor. 



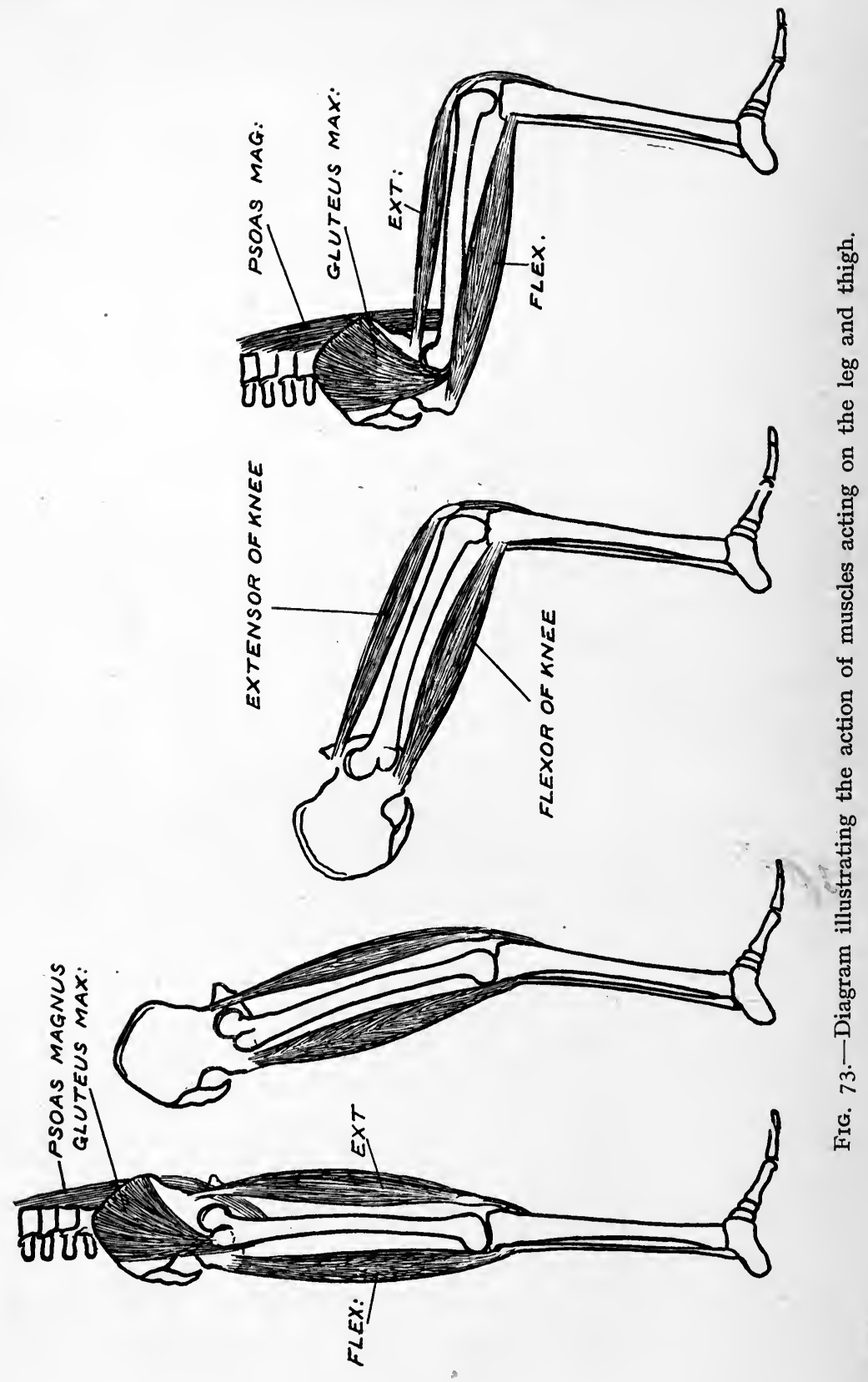

ซ్ 
The alteration of angle at the hip joint, of the trunk on the femur, is an independent action to that at the knee. The action of the flexors of the knee with the fixed point below is a flexion of the trunk and femur at the knee, irrespective of the angle between the pelvis, i.e. trunk and the femur at the hip.

These principles are illustrated in the accompanying diagrams (fig. 73). In addition to their actions as flexors of the knee these muscles can also produce a limited amount of rotation movement at the knee. This can only occur during the action of flexion, when the muscles are contracted, and not during extension of the knee, when they are relaxed and elongated. It is a limited rotation either in or out of perhaps not more than Io degrees, and can be demonstrated during flexion of the knee by placing a finger on the tubercle of the tibia. The chief muscle producing inward rotation is the popliteus, which corresponds to the pronator teres of the upper limb, and it is assisted by the gracilis, sartorius, semi-tendinosus, and semi-membranosus.

The outward rotator is the biceps. It has an advantage as an antagonist, in that its short head arises from the shaft of the femur. It has to be borne in mind that in chronic diseases of the knee joint, with backward displacement, there is frequently some outward rotation at the joint due to the action of the biceps-a point worth remembering in reduction of the displacement.

In the marsupials the semi-tendinosus and semi-membranosus have a much lower attachment on the tibia than in man (fig. 74), and their strength in the phyllophagous and arboreal koala is shown in the development of a bony eminence on the tibial shaft. In the platypus the gracilis is a broad fleshy flexor of great power and size, and beneath it lie the semi-membranosus, semi-tendinosus, and the adductores (fig. 75).

The most variable, or rather adaptable, of the flexors would appear to be the sartorius and the biceps. In reference to the retention in man of the peculiarly situated sartorius as a flexor we must remember that in the marsupial,- -and 
this can be well demonstrated in the kangaroo,-it is an extensor, being related to the quadriceps extensor at the inner side of the gristly patella (fig. 74). Hence the advantage of this muscle as a graft in cases of weakness of the

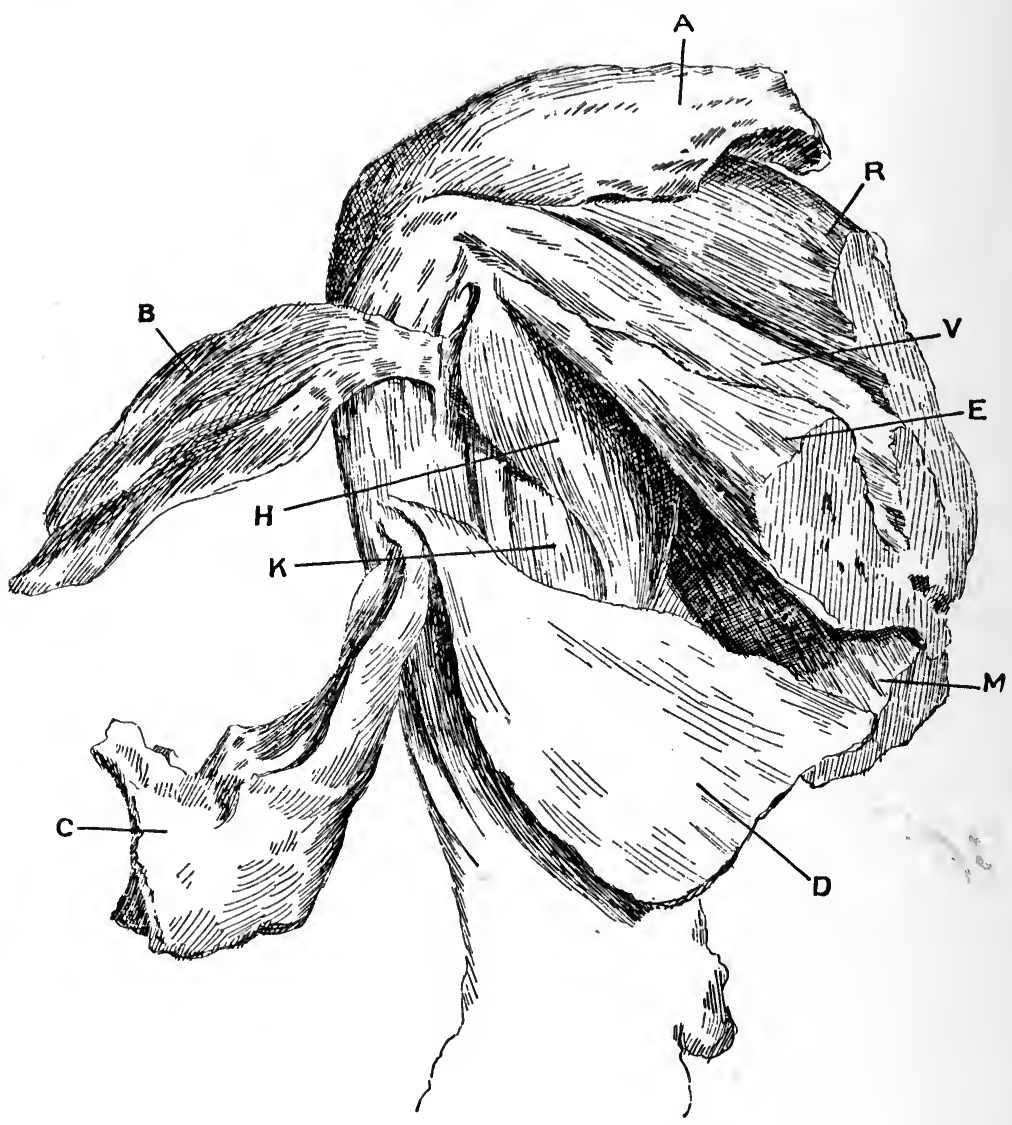

FIG. 74.-Dissection of inner side of knee.

Wombat (Phascolomys Mitchelli).

A, Sartorius. R, Rectus femoris. B, Gracilis. V, Vastus internus, E, Great adductor. M, Biceps cruris. D, Semi-membranosus. C, Semi-tendinosus, with biceps attachment. H, Gastrocnemius. $\mathbf{K}$, Tibialis posticus and deep flexor.

quadriceps. The biceps, both in the monotremes and marsupials, has a tendinous attachment along the whole length of the leg, and this is particularly well shown in the 


\section{MUSCLES ACTING ON THE LEG}

koala and the platypus (fig. 68, p.I35). Even in the kangaroo with its attempt at the erect posture this feature is present (fig. 69, p.I37). It is interesting to note that in the gorilla, the mammal whose attitude nearly approximates to the erect

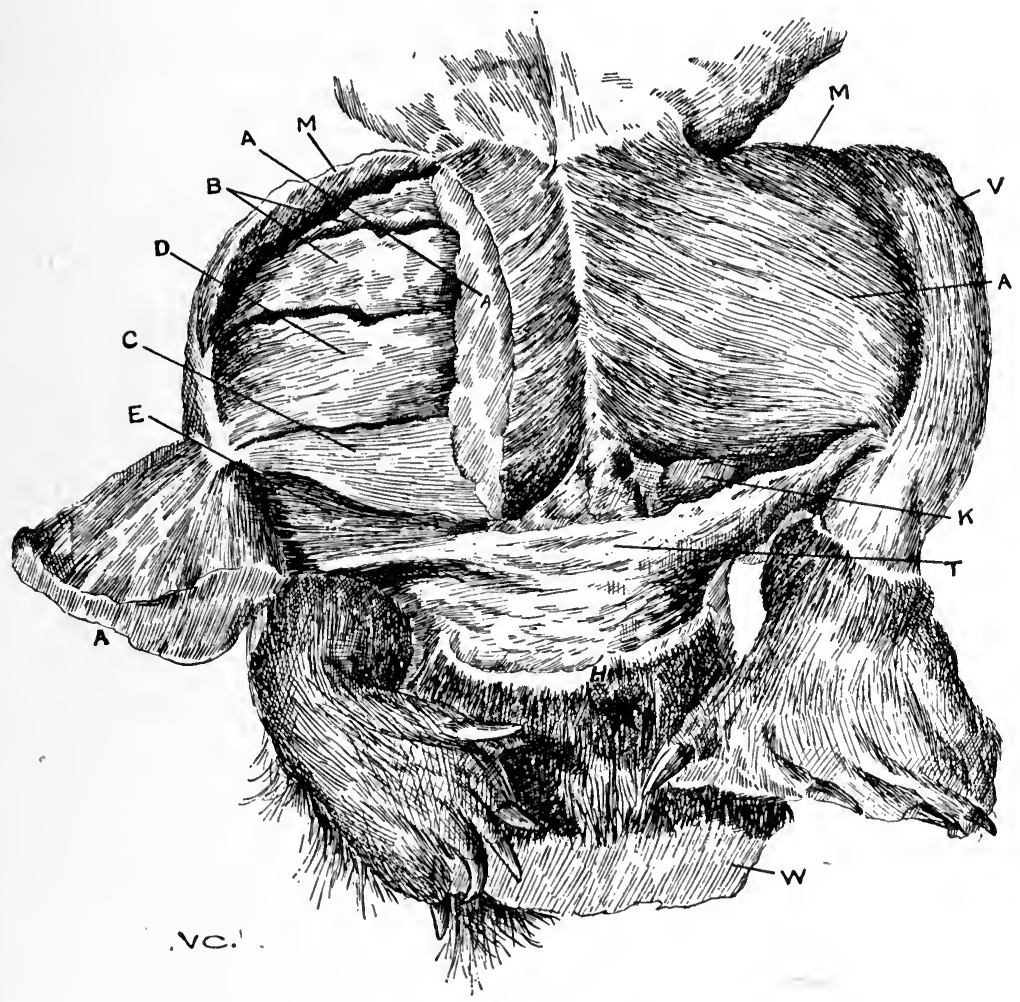

FIG. 75.-Muscles on ventral surface of thigh. Platypus.

H, Sphincter. K, L,eft Cowper's gland. W, Portion of tail. T, Adductor intertibialis. v, Anterior tibial muscles. M, Sartorius. A, Gracilis showing extensive insertion on leg. C, Semi-tendinosus. D, Semi-membranosus. B, Adductores.

posture of man, the portion of the biceps derived from the ischial tuberosity and inserted into the outer tuberosity of the tibia is more distinct than in man, from that portion having its origin at the linea aspera of the femur, which is inserted into the head of the fibula. 


\section{EXTENSORS}

These are the rectus femoris, two vasti, and the crureus, collectively known as the quadriceps extensor.

The rectus is superficial, lying on the front of the thigh with the vastus internus and vastus externus on either side. The crureus lies beneath these, being extensively related to the femur. The four muscles form a fleshy mass around the fore part of the thigh.

Rectus Femoris. - This muscle has a double head or origin-one head arising from the anterior inferior spine at the front of the ilium, and the other, or reflected head, arises from the dorsum of the ilium just above the top of the acetabulum. This latter is demonstrated by the student at the termination of the gluteal dissection. These two heads form a single tendon about 4 inches long. This is succeeded by the fleshy belly of the muscle, which, as it approaches the patella, becomes tendinous, and receiving laterally portions of the insertions of the vasti muscles is inserted with the tendon of the crureus into the upper border of the patella. In the muscle belly is a median tendinous line, towards which the muscle fibres converge, giving a bipennate arrangement to the rectus. This muscle is the only portion of the quadriceps arising from the pelvis and crossing the hip joint.

Vastus Externus or Lateralis.-This arises from the femur, being attached to the lower and fore part of the great trochanter-to the rough line leading from the trochanter to the linea aspera-and to the outer lip.of the linea aspera. The muscular belly is thick and fleshy, and the membranous tendon is inserted below into the outer margin of the tendon of the rectus and the upper and outer border of the patella.

Vastus Internus or Medialis.-This is not so large nor fleshy as the externus. In the dissecting room it is almost impossible to separate it from the crureus, the line of union being along the rounded border of the femur between the front and inner surfaces. It arises from the inner lip of the linea aspera on the shaft of the femur, extending from 
the anterior line between the two trochanters above almost to the inner condyle below. Its strong tendon is attached to the inner border of the rectus tendon and to the upper and inner margin of the patella. It is interesting to note that the fleshy fibres of the internus descend lower than those of the externus, and some are even inserted directly into the patella. This muscular extension gives rise to the fleshy cushion on the inside of the thigh above the knee.

Crureus (Vastus Intermedius).-This is a powerful muscle having a fleshy origin from the upper two-thirds of the shaft of the femur on the anterior and external surfaces. Apart from its close connection with the vastus internus it is also closely related to the vastus externus in the middle third of the thigh. Below, it is joined to the tendon of the rectus, and forms the deep portion of the quadriceps extensor tendon.

Insertion of the Quadriceps Extensor.-The tendon of the rectus, expanding at its termination, is joined on its under surface by the tendon of the crureus and laterally by those of the vastus externus and internus, the combined strong tendon being attached to the upper part and sides of the patella (fig. 76). From the lower border of the patella, what is generally regarded as the real tendon of insertion of the quadriceps or the ligamentum patellæ is continued over the front of the knee joint and is inserted into the tubercle of the tibia. In addition, from the lower fibres of the vastus externus and internus a fibrous sheetlike expansion is traced not only to the capsule of the knee-the so-called lateral patellar ligaments-but to the inner tuberosity of the tibia and the head of the fibula as well. The knee joint is thus protected at the sides as well as in front.

The Patella is usually regarded as a large sesamoid bone whose use is not so much to facilitate the movement of the extensor tendon over the condyles of the femur, since for this we have two bursæ-one between the ligamentum patellæ and the tubercle of the tibia, and the other between the crureus and the lower part of the femur-nor to protect 
the joint, which is an important service, but to strengthen the action of the extensor muscles. It is worth remembering however that the patella might be regarded as a flexible

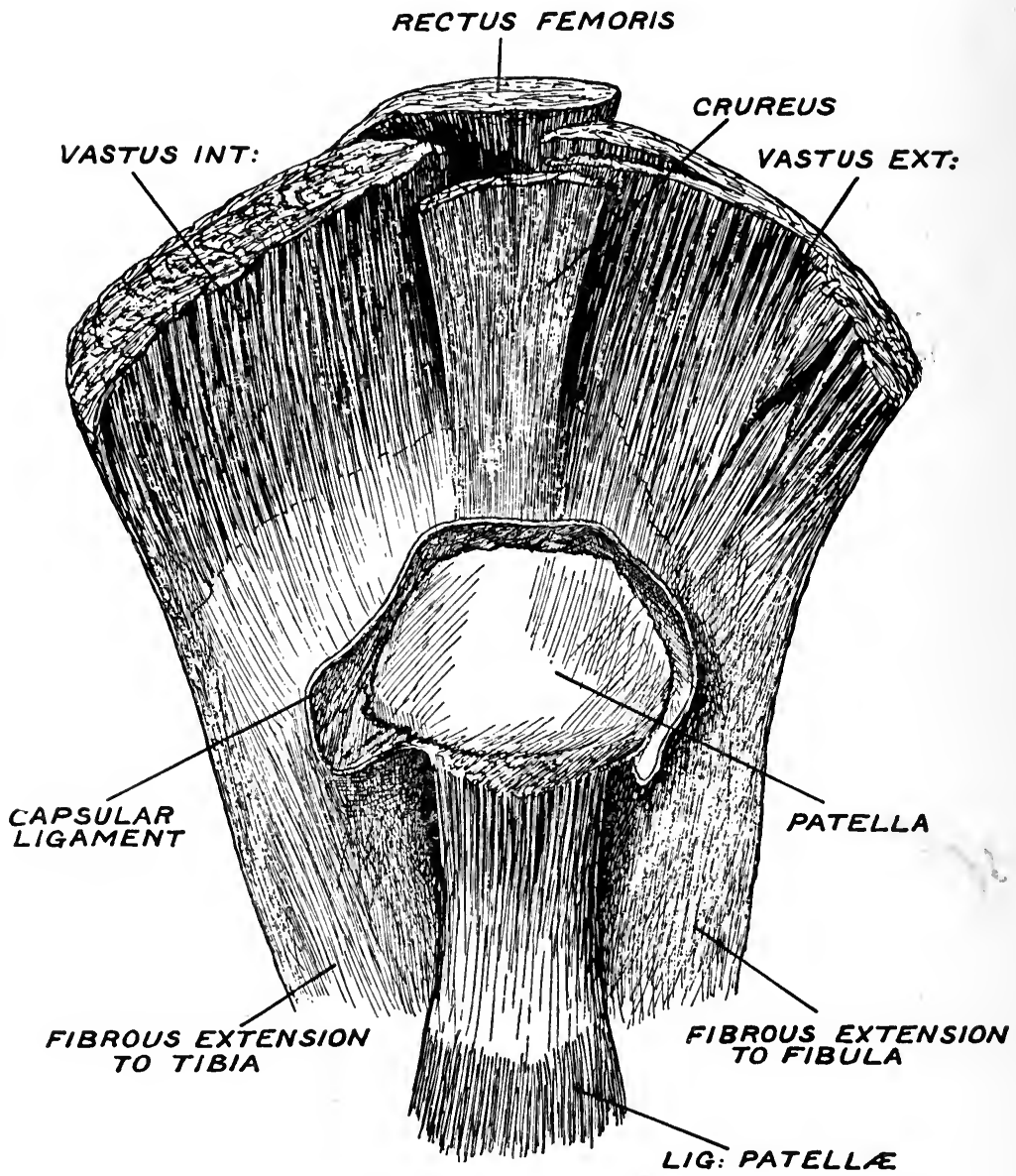

FIG. 76.-Insertion of the quadriceps extensor.

process of the tibia comparable to the olecranon of the ulna, the two being connected by the elastic ligamentum patellæ. A solid inelastic process like the olecranon would however be liable to direct injury, which is extremely rare in the case of the patella. 
Against this view, also, is the fact that the patella is developed as a separate bone in both monotremes at one end of the mammalian scale and in man at the other. In the mammalia I have never seen a patella joined normally to the tibia like the olecranon to the ulna, nor in marsupials as the wombat, where no patella is present, is there a process on the tibia comparable at all to the patella. The function of the patella is to improve leverage, and thus to increase the power of the quadriceps extensor muscle. Its function is practically that of a pulley gliding on the smooth surface of the lower end of the femur between the two condyles. By its means the motor force necessary to extend the knee, instead of acting on the centre motion in a straight line to the components of the articulation, acts at an angle, and consequently its range of power is increased.

The patella is a mechanical aid to the action of the quadriceps, providing as it were a new base for extensor action, since it lies between the motor forces and the insertion of their tendon. In the sitting posture, with the knees bent, the flexors being active and the quadriceps relaxed, the patella sinks in the hollow of the knee, only its upper third being related to the condyles of the femur. In the semiflexed position, when the quadriceps acts, the patella rises out of the hollow, and becomes more prominent. In complete extension, however, it is right at the summit of the knee, and only the lower facets are related to the articular surfaces of the condyles. In a case seen recently of a soldier whose left patella had been removed some months previously for compound comminution, it was interesting to note that whilst on the normal right side the patient when lying on the back was able to lift the heel off the bed with the knee fully extended; on the left side extension of the knee when the heel was raised was incomplete, being accompanied by a flexion of about 15 degrees. When lying on the right side the patient could elevate the left heel with the knee straight, and he was gradually brought from that position to the full dorsal, being finally able to raise the heel with the knee 
fully extended. Such a quadriceps, though at a mechanical disadvantage compared with the normal is sufficiently strong to enable the patient to walk. When walking is commenced, the knee should be at first extended, as, for example, by the use of a Thomas caliper splint. By means of a graduated hinge mechanism opposite the knee flexion can be increased-the body weight being thrown gradually on to the weakened articulation.

Functions of the Quadriceps.-The quadriceps extends the leg on the thigh, being the antagonist of the six flexors. Its action is one of the most powerful in the body. Part of the muscle, the rectus, arising from the pelvis, passes over the hip and knee, the remainder pass over the knee only. Owing to the pelvic origin of the rectus this muscle is said to act on two joints at the same time, to bend the thigh while it extends the knee, and reversely to bend the trunk on the thigh. The centre of motion or fulcrum for quadriceps action is not the hip, but the knee joint. Furthermore, we can readily extend a flexed knee with the hip extended, and can bend at the hip with the knee flexed. The quadriceps does not produce flexion at the hip joint, that action is solely produced by the ilio-psoas.

When the quadriceps takes its fixed point from below its action is to effect extension of the trunk and thigh on the leg at the knee joint ; irrespective of alterations of the angles of flexion of the components at the hip which depend on the ilio-psoas and gluteus maximus muscles. If, with the legs and feet fixed, we bend the body back at the knee the quadriceps will extend the body again to the erect position. Similarly, when we rise from the sitting posture, although the hip alters from flexion to extension owing to relaxation of the ilio-psoas and contraction of the gluteus maximus, it is the quadriceps which is the factor in extending the body on the leg at the knee joint. When we bend the body forward at the hip joint in standing, to pick an article off the ground, this action is produced by the contraction of the ilio-psoas and the relaxation of the gluteus maximus, 
It is rendered possible owing to extension at the knee by the contraction of the quadriceps, and relaxation and elongation of the hamstrings and the three other flexors.

These points I have dealt with more particularly under the actions of the knee flexors. The powerful action of the quadriceps in producing extension at the knee is the basic factor in the maintenance of the erect position of man, not only in standing but also in running and walking. The continual support of the superincumbent pressure made by the whole body depends on the quadricipes extensores. In their action, not only is it a question of the opposition of the flexors, but of body weight and gravity, hence their great relative size. Two centuries ago Cowper, the distinguished British anatomist, weighed the extenders and benders of the knee, and found that the extenders greatly exceeded the benders in weight. Thus in one body he found the extensors weighed $3 \mathrm{lbs}$. 6 ozs., and the flexors I lb. 7 ozs.; and in another the extensors weighed $4 \mathrm{lbs}$. $8 \frac{1}{2}$ ozs., and the flexors 2 lbs. 6 ozs.

When we stand erect on both legs each quadriceps, though contracted, is not at its maximum effort; and owing to the fact that the patella appears loose some anatomists have stated that the knee extension is not dependent on muscular aid. The knee extension does depend on quadriceps contraction-the position of the patella tells us that. A child whose quadriceps is paralysed cannot stand if the knee be placed in the position of extension. The reserve force of this muscle is brought into action if we stand on one limb, when the quadriceps will be felt further to contract and tighten. The strength of the quadriceps is also shown in its ability to produce a fracture of the patella. This not infrequently occurs when a person is going upstairs. The balance is for some reason upset while the knee is semi-flexed, and a sudden effort is made to tighten the joint in extension. The result is that a violent spasm of the quadriceps occurs which is not associated, as in ordinary circumstances, with a pari passu relaxation and elongation of the flexors. These are taken 
unawares, and the force of the quadriceps action falls on the patella, which is frequently unable to bear the strain.

\section{Paralysis of the QUAdriceps Extensor}

That the quadriceps is the great factor in maintaining the erect posture is well illustrated in cases of infantile paralyses affecting the lower limb. In any large city numerous cases are to be met with, ambulating on crutches with a dangling limb, unable to support half the weight of the body owing to a paralysed quadriceps extensor(fig.8r, p. I66). Such patients may be able however to stand immediately if the knee be stiffened by means of a splint placed behind the knee and bandaged to the limb; in other words, by the creation artificially of a contracted quadriceps. That the term "quadriceps extensor" has a different value in man able to adopt the erect attitude, and in a lower mammal unable to stand erect, was impressed on me by a study of cases of poliomyelitis. Cases of quadriceps "paralysis" were met with in this disease in which the patient was unable to stand, or when lying in bed to raise the heel with the knee extended. When he lay, however, on the side opposite to that which was affected and the knee was acutely flexed, the leg could be extended with ease.

Because the patient is unable to raise the heel off the ground with the knee extended when sitting on a chair-is unable, that is, to do Ioo units of work-we are not justified in calling the quadriceps " paralysed." On the same method of reasoning the quadriceps tested with the patient in bed and with the knee acutely flexed might be regarded as normal, since it can perform the function of the quadriceps as given in text-books; that is to say, extension at the knee. These phenomena admitted of only one explanation-namely, that the disease was revealing functional stages in the evolution of muscle. They showed clearly that muscle action was a complex functionone made up of several separate activities - and hence the term "paralysis of muscles" is really referable to the loss 
of some, not necessarily all of the functions. The recovery of function, like the loss, follows in an ancestral or evolutionary sequence. This applies equally to the deltoid as to the quadriceps. In the case of knee extension for the erect posture new muscles have not been called into being-orthograde functions have been superimposed on muscles performing in other mammals plantigrade motion.

Charles Bell taught us that the anterior cord presides over muscular action, and Marshall Hall that movement can occur reflexly from irritation independently of volition. A study, however, of cases of infantile paralysis in man, regarded as the most advanced mammalian type, demonstrates the fact also that the anterior cord not merely presides over " muscular action," but recognizes the components of which that function is made up; and that these cell-controlled components always bear a definite sequence one to another. Though similarly placed in an anatomical sense the action of the quadriceps is vastly different in platypus and in man. The quadriceps in platypus responds to the anatomical test of extension of a flexed knee, but that is a different function from that of a quadriceps, which will allow of the orthograde posture. The quadriceps of the ape has a more complex function than that of the platypus, and so, similarly, has man's compared with the anthropoid. A patient who can raise the heel off the ground when sitting with the knee extended will be able to extend the acutely flexed knee in bed; but the patient who can do the latter need not necessarily be able to do the former. When, therefore, we are told that the quadriceps is paralysed merely because the patient is unable to raise the heel off the ground, we are told something which is biologically untrue. This is a question apart altogether from volition, since in a condition like infantile paralysis, we are dealing with a disease in which generally cerebration is unaffected.

A study of the anthropoids at any menagerie shows the reluctance with which the fore limb is dispensed with as a means of support. The ape prefers the knee slightly bent, with the balance afforded by one or both fore limbs. To 
assume the orthograde posture is an effort, and a still greater one while so supported to raise the fore limb above the head. The stiffening of the knees by the quadriceps which has enabled man to stand and walk, and the ability not only to dispense with the fore limb for support, but to elevate it when erect above the head, are late acquisitions in man's evolution, and are hence unstable.

\section{Method of Resting a Paralysed Quadriceps Extensor}

The most frequent cause of this condition is poliomyelitis in children. In this disease it is essential at the outseteven if only one limb be affected-to rest the two, as pelvic tilting, the result of over-action of one side, easily occurs, and once having occurred is never overcome. It is also difficult at the outset to make sure whether the abdominal muscles are affected, and furthermore, only in this way can we prevent undue rotation of the hip, and abduction, or adduction, to which there is so great a tendency. Hence the importance of noting the relationship of the two anterior superior spines to the umbilicus, or more correctly to the mesial plane. The spines should be on the same horizontal level. This can be immediately effected temporarily by means of two padded boards placed behind the extended knee from the upper third of the thigh to the lower third of the leg, with a connecting piece across the middle-or two long back splints with foot-pieces, joined together-and permanently effected by the simple abduction splint (fig. 7 I, p. I40). The quadriceps is in a position of anatomical rest because its opponents, the flexors, are in a position of relaxation and elongation. To prevent overstretching of the flexors a small cushion should be placed under the knee. With the feet elevated on a pillow strain is taken off the ilio-psoas and the rectus which arises from the pelvis. Anatomically, then, the lower limb, like the upper, can be easily and should be immediately rested. If these details are attended to we have gone a long way on the road to secure a recovery. Through failure to effect anatomical rest at the outset 
can be largely ascribed the bad results met with in infantile paralysis.

Method of Re-education for a Paralysed Quadriceps

A paralysed quadriceps should be handled with the greatest gentleness, and when taken off the splint the limb should occupy at first the position it had when supported

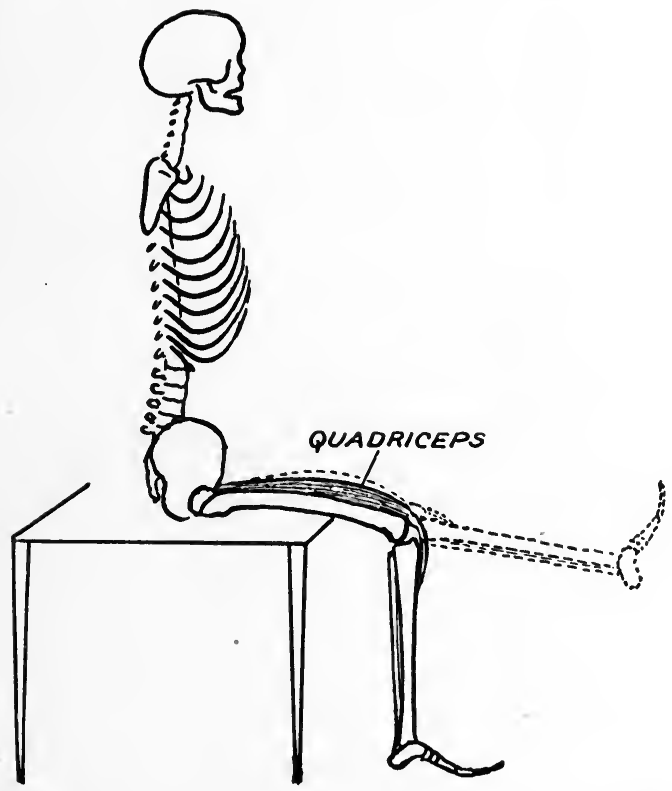

FIG. 77.-Complete recovery of quadriceps action (maximum work). An erroneous method of testing for paralysis.

by the splint. The knee should not be bent up nor allowed to hang over the edge of the bed. We should exercise just as much care as in the case of a fracture of the femur. It is best for the patient to rest on a firm bed, so that movements can be carried out without disturbance. A sheet of cardboard should be placed under the $\operatorname{limb}$ to obviate all resistances to movement. The maximum worknamely, Ioo units-is represented by the ability to stand 
erect with the knees straight, or to raise the heel off the bed when lying with the knee extended, and similarly when

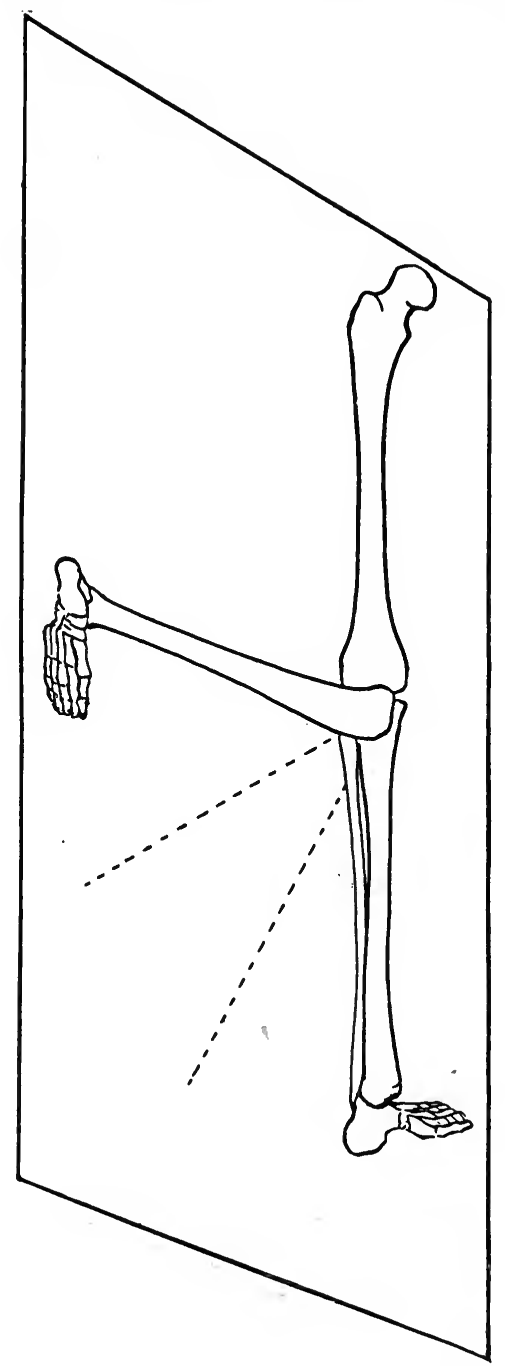

FIG. 78.-Commencing re-education of the quadriceps.

sitting on a chair (fig. 77). The minimum is represented by the ability to straighten the knee without abduction, with the patient on the back and the knee slightly bent, 
actively or passively. On flexing the leg, for example, one inch, it may be noticed that the limb falls straight; or, if bent, say threeinches, the patient may be unable to straighten the knee, but can prevent the limb abducting; that can be regarded as evidence of recovery. The flexion is gradually increased, the fall becomes gradually replaced by a definite push, till finally the patient holding the knee in the position of acute flexion, without abduction or adduction of the thigh, is able to strâighten the limb. It may be necessary to initiate action with the patient lying on the opposite side, and not on the back, beginning the push with a two-inch flexion, and in this early movement hip action is unhindered. An early sign of recovery is the power of adduction or abduction of the whole limb when on the back with the knee straight, especially the latter, and progress can be stimulated by bandaging a splint to the back of the knee.

Our next endeavour is to secure extension from full flexion when lying on the opposite side, with the thigh fixed (fig. 78). This is accomplished by beginning with, say, a 3-inch flexion and gradually increasing, always commencing each day, however, at the 3 -inch stage. It is wrong practice to flex the knee beyond a point at which

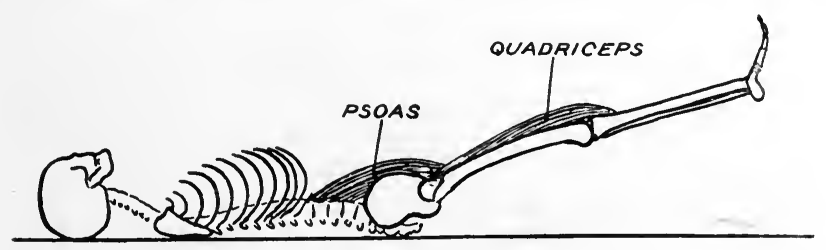

FIG. 79.-Complete recovery of quadriceps. Ioo units of work, or maximum.

extension effort becomes manifest. The action is aided by allowing the patient to flex and extend the knee when on the side, but with the thigh free. The time now comes when our attention is directed to the power of raising the heel off the bed from the hip with the knee extended (fig. 79). To our surprise the patient, when lying on the sound side, may lift the affected limb in one piece and swing it 
forwards and backwards with ease. This is an action of the ilio-psoas muscle, aided by the abductor of the thigh, but is only rendered possible by the action of the quadriceps extensor in keeping the knee stiff. If unable to do this we

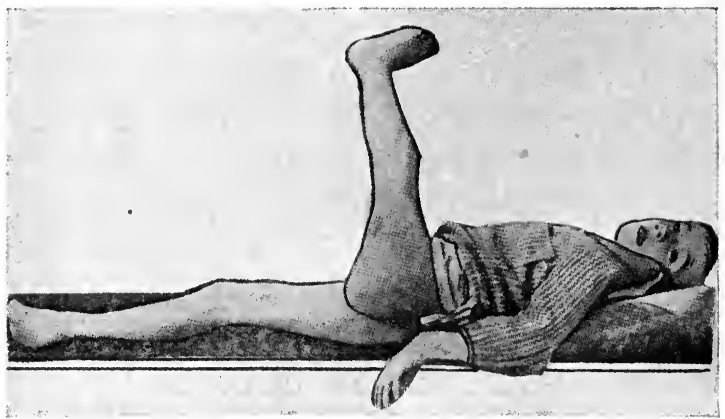

FIG. 80.-Almost complete recovery of quadriceps (same patient as fig. 8I).

can assist the patient to initiate and practise the movement with the knee stiffened by a cardboard or wooden splint behind the joint-that is to say, we produce an artificial quadriceps. Objection may be raised that the elevation of the limb in one piece when on the side is dependent on

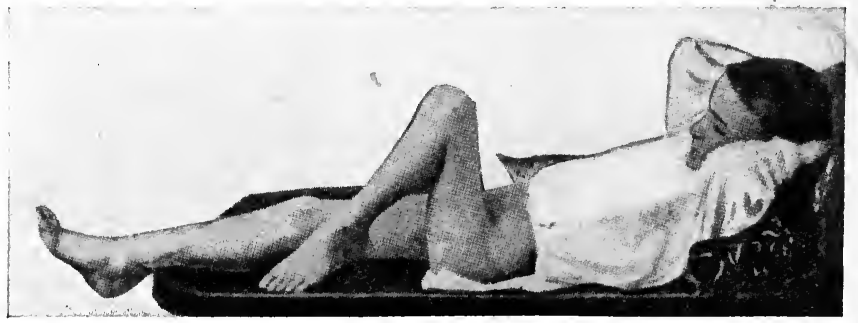

FIG. 8I.-Contracted knee of ten years' duration, the result of contraction of flexors owing to paralysed quadriceps (same case as fig. 80).

the stiffening action of the lateral ligaments. It is, however, a distinctly muscular action, and ligaments do not produce motion.

This can be proved in the case of a severe quadriceps paralysis, when the patient with a sound ilio-psoas will make 
unsuccessful efforts to raise the heel when on the side-the knee remaining bent; but when the splint is applied behind the knee will lift the limb with ease. Although able to raise the limb in one piece when on the side, yet when turned on the back, this, which is the maximum effort, is impossible. The graduated method is again applied. We gradually alter the tilt of the body (fig. 80), which can be done by means of a pillow, from the lateral to the supine position, till finally our maximum of roo units of work is reached. If, when seen, the knee has been bent owing to over-strong hamstrings (fig. 8I), it should first be gradually straightened before true function is commenced. There should be no flexion at any time beyond the point at which extension effort has become manifest. 


\section{CHAPTER IX}

\section{MUSCLES ACTING ON THE FOOT (GREAT SCIATIC NERVE)}

THE muscles acting on the foot are eight in number, and produce the movements of flexion or upward movement (dorsi-flexion), and extension or downward movement (plantar flexion); also of adduction, inversion, or turning the foot in; and abduction, eversion, or turning the foot out. Flexion and extension take place at the ankle joint, and inversion and eversion chiefly in the tarsal articulations, and more particularly in the mid-tarsal joint. The muscles are :

\section{Flexion}

Tibialis anticus

Peroneus tertius

Peroneus brevis
Extension

Gastrocnemius

Soleus

Plantaris

Tibialis posticus

Peroneus longus

Inversion

Tibialis anticus with flexion

Tibialis posticus with extension
Eversion

Peroneus longus with extension Peroneus brevis with flexion Peroneus tertius with flexion

\section{FLEXION AND EXTENSION OF THE FOOT}

\section{FLEXION}

Tibialis Anticus.-This muscle is placed on the outer side of the tibia arising from the lateral tuberosity or condyle and upper two thirds of the shaft of that bone. The belly is adherent to the deep fascia covering it, and terminates about 4 inches above the ankle in a tendon which, crossing the ankle joint, is inserted on the inner surface of the first 
or internal cuneiform bone and on the base of the metatarsal bone of the great toe. Tendon comprises half the length of this muscle.

Peroneus Tertius. - This is usually described as a special-

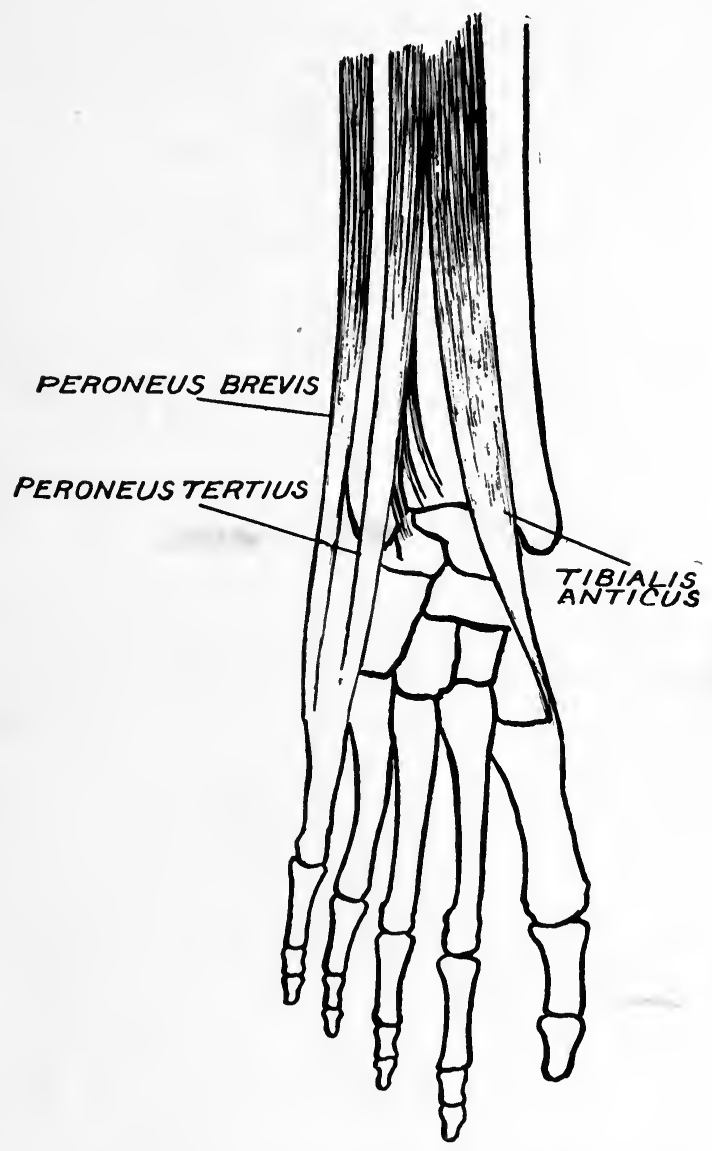

FIG. 82.-The benders (dorsi-flexors) of the foot.

ized portion of the extensor longus digitorum, not specially innervated, and not always present. When present it represents the outer part of the extensor brevis digitorum, retaining its old mammalian origin from the leg, although the main part of the muscle has migrated to the foot. Its 
tendon passes in front of the outer malleolus, while those of the peroneus longus and brevis pass behind.

It arises from the lower third of the anterior surfaces of the fibula, and its tendon is inserted into the dorsal surface of the base of the metatarsal bone of the little toe. There is no slip in the fore limb corresponding to the peroneus tertius, and this muscle was regarded by Owen and others as peculiar to the human leg. In the leg of the wombat, however, there is a well-defined fleshy muscle corresponding to the peroneus tertius, and having an insertion on the dorsum of the foot laterally. This sends a tendinous slip to extend the little toe.

Peroneus Brevis.-This arises from the lower two thirds of the outer surface of the fibula, and an important point is that it is fleshy lower down in the leg than the longus. It passes with the peroneu; longus behind the outer malleolus which forms a pulley for the improvement of their action, especially of the longus, and its tendon, running along the outer side of the os calcis, is inserted into the tuberosity and dorsal surface of the base of the metatarsal bone of the little toe. The insertion of this muscle is dorsal and not plantar. It is always demonstrated in dissections of the dorsal aspect and not in those of the sole of the foot. Expansions from the tendon, just before its insertion, I have always found to be directed towards the medial aspect dorsally and not towards the plantar aspect. This point is of the utmost importance in a consideration of the action of the peronei.

\section{Extension}

Gastrocnemius.-This is the great calf muscle, and arises by two tendinous heads-an inner and an outer. The outer head arises from the upper and back of the lateral or outer condyle, while the inner or larger head arises just above the inner or medial condyle, between it and the adductor tubercle. Each tendon is connected with a fleshy belly, and the two bellies being connected firmly about the middle 
of the leg are succeeded by a large flat tendon, broad above and narrowing below-the superficial portion of the tendo achillis-and this is inserted into the lower part of the posterior surface of the calcaneus or os calcis. A bursa to facilitate action lies beneath the tendon at its insertion. This muscle, unlike the soleus, crosses the knee joint.

Soleus.-This lies beneath the gastrocnemius, and arises by two heads also, one from the head of the fibula and upper third of the back of that bone, and the other from the middle third of the inner or mesial border of the tibia. The tibial origin would appear to be peculiar to man. Even in the gorilla the soleus has an origin from the fibula only. Some fibres also arise from a tendinous arch between the two heads. The fibula head is large and round and the tibial head smaller and flatter. They are succeeded by a fleshy belly, which about the middle of the leg is succeeded by a tendon, and this blending with that of the gastrocnemius forms the greater part of the tendo achillis. This, as stated above, is inserted into the posterior or dorsal aspect of the os calcis. As the powerful tendo achillis approaches the heel it narrows, but on reaching the bone it spreads out to take a firm grip. This muscle does not cross the knee joint. The calcaneus or os calcis can be regarded as a point of support for the whole foot, and through it is exercised the lever power by which the superincumbent body weight is raised in the peculiar bipedal walk of man. Its strength and backward development relate purely to increase of power, and depend on muscular development. There is a distinct difference in the size of the os calcis in African races, whose members walk barefooted and carry heavy burdens, and the modern European. By means of a single tendon of insertion nature is able to concentrate all the force of the powerful calf muscles on the calcaneus.

Plantaris. - This muscle is often wanting. It is so called as it was thought it was continued on as the plantar fascia. Comparative studies show that this fascia results from the retrogression of the plantaris. Its slender fleshy belly is 
about three inches long, and is traced to the lateral condyle of the femur. Its narrow tendon runs along the inner margin of the tendo achillis to the posterior surface of the calcaneus. This muscle crosses the knee joint. It has never been detected, as far as I know, in the gorilla.

Tibialis Posticus.-This is the most deeply seated muscle in the leg, and arises below the knee joint from both the

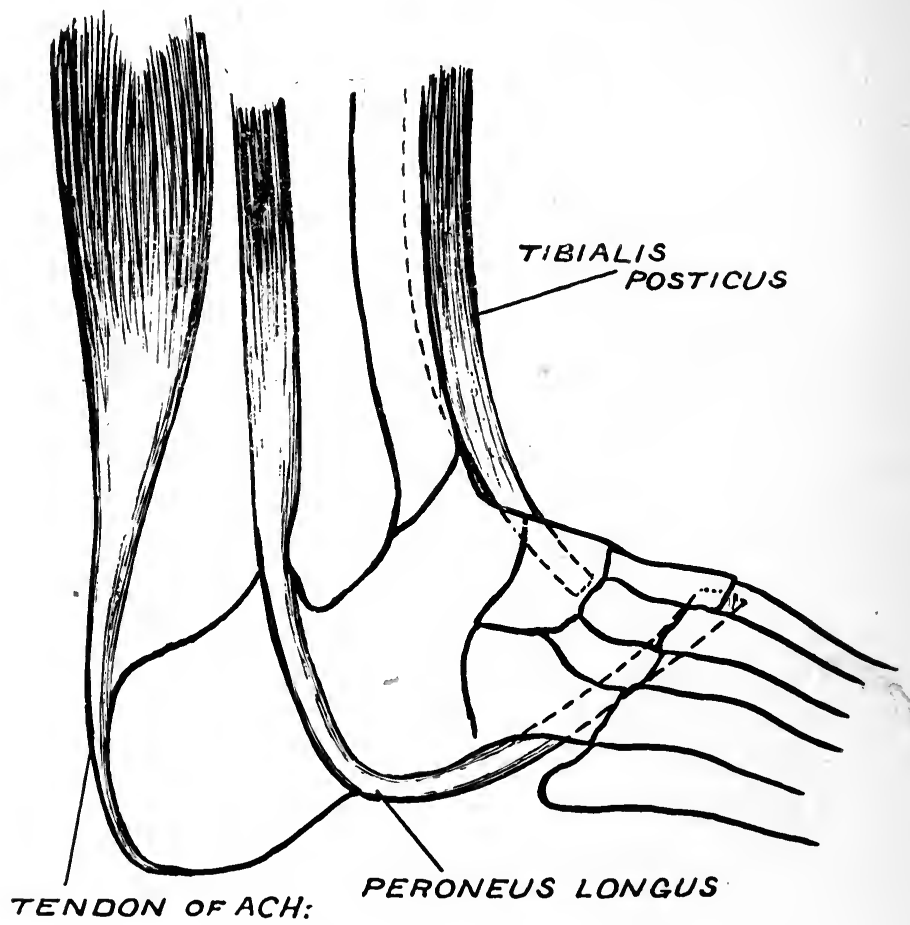

FIG. 83.-The extenders (plantar-flexors) of the foot.

tibia and fibula, as well as from the interosseous membrane between the bones, and from the deep fascia over it. Its attachment to the back of the fibula is from the upper two thirds of the inner surface, and to the tibia from the dorsal or posterior surface below the oblique line. The muscle belly terminates in a tendon, and this passes behind the inner malleolus, which acts as a pulley for it like the 
outer malleolus does for the peronei. The principal insertion of the tendon is to the plantar surface of the tubercle of the navicular or scaphoid and the adjacent internal cuneiform bones; but, in addition, fibrous expansions are given off to the two outer cuneiform bones-cuboid and sustentaculum of the calcaneus - and also to the second, third, and fourth metatarsal bones. Its insertion is expanded much more than the peroneus longus, and owing to the firm, spreading, tentacle-like grip it has of the sole of the foot the insertion has been likened to a hand.

Peroneus Longus.-The muscle lies superficial to the peroneus brevis, and arises from the head and upper two thirds of the outer surface of the fibula. Its tendon passes with that of the brevis behind the outer malleolus, which forms a pulley round which the tendon plays, and so has its function increased. Running on the outer side of the os calcis to the cuboid it enters the sole of the foot, across which it runs obliquely to be inserted into the base of the metatarsal bone of the great toe, and to the internal cuneiform bone as well. Both behind the outer malleolus and on the outer side of the os cuboid, where the tendon alters its direction, it is thickened. Whilst the outer malleolus acts as a pulley for the muscle, it is interesting to note that at the os cuboid, where it is entering the sole of the foot, a fibro-cartilage is formed in the tendon which plays by means of a bursa over a smooth tubercle on the cuboid bone.

\section{MUSCULAR ACTIONS}

\section{Flexion and Extension}

In the movements of pure flexion and extension of the foot, without inversion or eversion, bending (dorsi-flexion) is produced by the tibialis anticus, peroneus tertius, and peroneus brevis, and extending (plantar-flexion) by the gastrocnemius, soleus, plantaris, tibialis posticus, and peroneus longus. The front of the leg and dorsum of the 
foot belong morphologically to the general extensor surface of the limb, corresponding with the back of the forearm and the dorsum of the hand. The back of the leg and sole of the foot correspond with the front of the forearm and palm of the hand, so that what we call flexion is really over-extension. It is usual, however, and it will be followed here, to use the term flexion for the movement by which the dorsum of the foot is bent towards the front of the leg; and extension to the movement by which the sole of the foot is directed towards the back of the leg. In the movements of flexion and extension at the ankle three principles of leverage are illustrated.

(a) If we raise the foot from the ground and then extend it so as to point the toes, we illustrate a lever of the first order, the fulcrum or ankle joint lying between the power of the tendo achillis at the calcaneus and the weight or front of the foot which offers the resistance (fig. 2, p. I0).

(b) When we stand on tip-toe we illustrate the second variety of lever-the fulcrum being at the ball of the toes; the power is applied by the muscles of the calf at the os calcis, and between the two is the weight to be raised, which is that of the body communicated through the tibia (fig. 3, p. II).

(c) If we raise one foot from the ground and flex it we illustrate a lever of the third order, the power represented by the insertion of the tibialis anticus, peroneus tertius, and peroneus brevis, lying between the weight, or front of the foot, and the fulcrum at the ankle-joint.

The chief flexor of the foot on the leg is the tibialis anticus and the chief extensors are the gastrocnemius, soleus, and plantaris acting through the tendo achillis. The disparity in size between the extenders and the flexors is very evident, and while the tibialis anticus may weigh only $5 \mathrm{oz}$., the gastrocnemius and soleus may weigh five times that amount.

The muscles acting through the tendo achillis on each side, when they take their fixed point above, are important muscles in running, walking, jumping, and standing on tiptoe, in which their action is against gravity and the body 
weight, hence their size and strength. When we stand, their fixed point is at the heel, and they are important factors in keeping the body from inclining the tibia and fibula forwards at their articulation with the astragalus. These muscles (fig. 63, p. 128) thus steady the leg on the foot

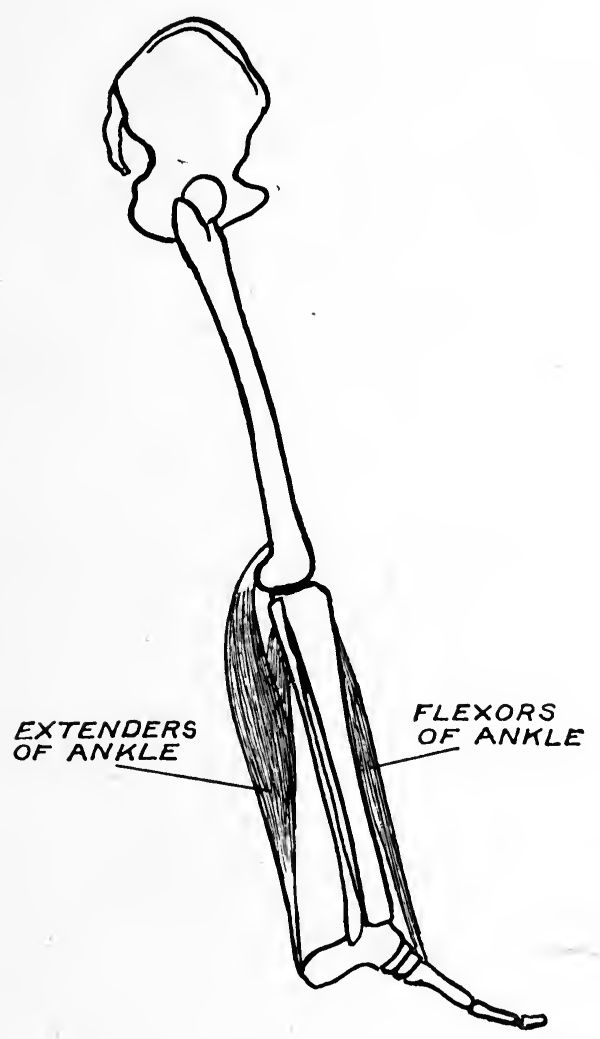

Fig. 84.-Extension backwards of the rigid body at the ankle joint.

and help to prevent the body from falling forwards. The gastrocnemius has been described not only as an extensor of the foot, but as a flexor of the knee as well. It does not act on the knee joint. Its contraction is not necessarily associated with relaxation of the quadriceps. That is only associated with muscles having the same 
centre for motion, the flexors of the knee. We can extend at the ankle in either the position of flexion or extension of the knee. The best illustration of the action of the flexors and extensors of the ankle when they take their fixed point from below, viz. the foot, is seen in certain acrobats. These, when standing with the hip and knee joints in the position of extension, can bend the body forward in one piece at the ankle, so as to pick an object off the ground (flexors of the ankle), and can extend the body at the ankle back to the erect position (extensors of the ankle). Thus we see the necessity for strong extensors which can bring the body back against gravity-the soleus gripping the tibia and fibula, and the gastrocnemius the femur. From the position of equilibrium at the ankle in the erect posture, in which the powerful extensors of the ankle antagonize not only the flexors, but also gravity, the calf muscles are able to produce a limited amount of extension backwards of the rigid body at the ankle joint, as shown in fig. 84. This, like so many of the reverse actions of the lower extremity, is best seen in athletes.

In talipes calcaneus (fig. 85B), the result usually of poliomyelitis in childhood, the functions of the muscles of the calf (extenders) are weakened and the unrestrained flexors contract, producing the attitude of flexion at the ankle joint. In this condition there may only be an acute angle between the dorsum of the foot and front of the leg, and in some severe cases the two may actually be in contact. The patient walks on the heel. In talipes equinus (fig. $85 \mathrm{~A}$ ), which is usually also the result of poliomyelitis, the opposite condition is present. The flexors are paralysed, and the extensors contract and shorten, resulting in the tense unyielding tendo achillis requiring operative lengthening to produce muscular equilibrium, and so to overcome the deformity. The heel is drawn up, and in severe cases may actually form a straight line with the leg. The patient walks on the balls of the toes, which, in walking, form a right angle with the foot. 


\section{INVERsion AND Eversion}

(a) Inversion.-When we speak of the tibialis anticus, peroneus tertius, and peroneus brevis as being flexors of the foot, and the tibialis posticus and peroneus longus as being extensors, we mean that as regards eversion and inversion of the foot, these muscles are then in a state of equilibrium. They are evenly balanced as regards those particular functions, so that we thus obtain a pure flexion and extension at the ankle without any. lateral deviations. Why we have two inverters (the tibialis anticus and posticus), and two principal everters (the peroneus longus and brevis), is to allow of either inversion or eversion in the position of either flexion or extension of the foot. Turning the foot, either in or out, can take place in the position of flexion or extension, and each movement must be evenly opposed. Inversion with flexion is produced by the tibialis anticus, and eversion in the flexed position by the peroneus brevis, assisted by the peroneus tertius when present. Inversion with extension is produced by the tibialis posticus (fig. 87, p. I94), and eversion with extension is produced by the peroneus longus. These movements would appear to be associated with the arboreal habit of man's ancestors to which Professor Wood Jones has directed our attention.

If when sitting we raise the foot off the ground and extend it at the ankle, the tibialis anticus and the other flexors relax and elongate, and the extensors contract and shorten. If we then invert the foot in the extended position, this is due to further contraction of the tibialis posticus, and its tendon can be felt to rise up close to its insertion at the navicular or scaphoid bone. It is interesting to note that in cases of acquired talipes equinus (fig. $85 \mathrm{~A}$ ), with the patient walking on the balls of the toes, it is rarely necessary to do more than elongate the tendo achillis. Should there be any inversion of the foot (varus) it is the tibialis posticus that requires division. If, when sitting, we raise our foot off the ground and flex it 
at the ankle, the tibialis anticus, peroneus tertius, and peroneus brevis contract and shorten; the muscles uniting at the tendo achillis, together with the peroneus longus and tibialis posticus, relax and elongate. If we now invert the foot the hollow over the tibialis anticus on the front of the leg, associated with its contraction for flexion, becomes distinctly deepened and the tendon stands out more prominently on the inside of the foot. In a condition of equilibrium of the muscles acting on the foot, in which the latter is midway between flexion and extension, and between inversion and eversion, inversion of the foot, in which the inner border is raised and the sole looks inward, would be the result of the combined action of the two tibial muscles.

In an arboreal animal like the marsupial koala, in which the big toe can oppose like a thumb and the fibula approaches the tibia in size, the importance of good inversion and eversion of the foot is obvious. The evolutionary evidence derived from a study of the foot of the kangaroo especially seems to show that the tibialis anticus or flexing inverter is "selected" in preference to the tibialis posticus or extending inverter. In the saltatorial kangaroo, which may reach 8 feet in height and whose leap may vary from 25 to 30 feet, eversion of the foot -which practically consists of one main toe-is prevented by the retention of a very powerful muscular tibialis anticus. In this animal I have often found the posticus to be absent, and when present to consist of nothing more than a tendinous shred about the thickness of strong cotton. In the kangaroo the fibula can be regarded as non-existent as a separate bone. For its lower two thirds it has become attached to the tibia, in which it is becoming incorporated, just as the appendix is in the wall of the ileum in the wombat, and the right adrenal is in the liver of the Australian phalanger. In cases of congenital defects of the fibula in the human subject, which are associated with eversion of the foot, the condition in the kangaroo's foot points the way towards treatment (fig. 5, p. 32). A bone which in the human species is obviously undergoing disappearance is scarcely one in which 
to expect reparative changes from bone grafting. The indication is to develop the tibialis anticus as powerfully as possible. This can be done by resting the foot at night in a shoe in which the member is flexed and inverted, and by encouraging the patient to walk on the outside of the foot by having the inside of the boot arched at the instep, and the sole and heel raised on the inner side. This method I have seen successfully carried out in several cases.
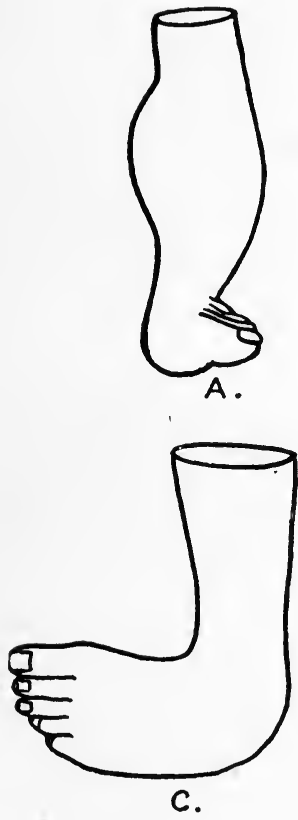

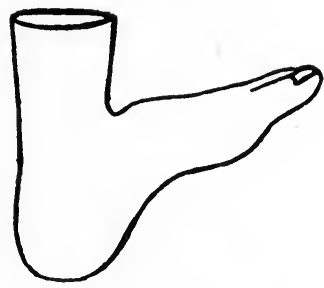

B.

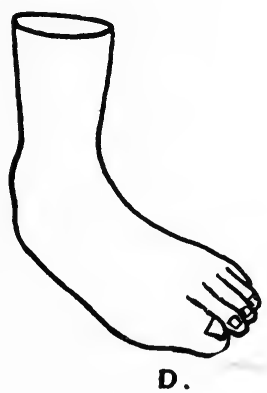

FIG. 85.-Acquired deformities of the foot. A, Equinus. B, Calcaneus. C, Varus. D, Valgus.

(b) Eversion. - In a condition of equilibrium of the toot as regards eversion or inversion, the peroneus longus, which is inserted on the sole of the foot, acts as an accessory extender, and the peroneus brevis, which is inserted on the dorsum of the foot, acts as an accessory bender. The peroneus brevis does not, as is usually taught, help to extend the foot on the leg, antagonizing the tibialis anticus and peroneus tertius, 
which flex. On the contrary, it is an accessory muscle to these latter. If we raise the foot off the ground and flex it, this latter movement is performed by the tibialis anticus, peroneus tertius, and peroneus brevis; the tendo achillis muscles and the peroneus longus and tibialis posticusbeing relaxed and elongated. If now we evert the foot, this eversion is produced by further action of the peroneus brevis, assisted by the tertius, which are both in a physiological state of contraction. If we raise the foot off the ground and extend it, this movement is performed by the muscles of the tendo achillis and the tibialis posticus and peroneus longus - the tibialis anticus, peroneus tertius, and peroneus brevis being in a physiological state of relaxation. If now we evert the foot, this action is produced by the peroneus longus, which is in a physiological state of contraction. Thus the two peronei, though inserted differently, produce in different states of the ankle a similar result-the one turning the outer edge of the foot up and the other turning the inner edge down. If the foot be in a condition of equilibrium as regards flexion and extension, eversion of the foot is produced by the combined action of the peronei.

When they take their fixed point from below the peronei and the tibiales help to maintain the erect posture by steadying the leg on the foot, which is especially evident when we stand on one leg-the opposing and balanced groups not only strengthening the ankle joint, but preventing the body from falling to one side or the other. The peroneus longus, by its insertion at the ball of the great toe enabling the inner edge of the foot to be turned down to the ground, is especially serviceable when we stand on tip-toe, whereby, with the feet separated and everted and the ankle extended, the weight of the body is transmitted to the ground mainly through the ball of the big toe. Hence in man the importance of this muscle in walking, running, and jumping. Its action is especially well exemplified in dancing and skating. It is at the ball of the big toe that severe pain is often felt after a long walk. 
From the point of view of selection, however, it would appear that the action of the peroneus longus is of less importance than that of the peroneus brevis. The brevis in man is muscular, practically down to the malleolus, and just above the malleolus its size is about twice that of the longus, which hugs it closely. In the koala, with its opposable big toe, the longus has a well-defined use, being traced to the hallux and acting as an adductor ; and in the anthropoid it is a grasping muscle, acting as a flexor of the metatarsal bone of the hallux. In the wombat, with a diminutive big toe, we have a relative diminution in size of the longus, which is plainer seen in the kangaroo. Here the peroneus longus is practically non-existent, so it cannot be of the importance in retaining the foot on the ground previously imagined. In this animal the longus attachment is retreating laterally, and a retreating muscle is a weakening one; and this is further contrasted by the greater belly muscularity of the brevis. In the koala I have noted laterally on the dorsum of the foot two peronei accessory to the peroneus brevis. In a case of valgus (eversion) (fig. 85D), in which the patient walks on the inside of the foot, the condition at the ankle being neither that of extension (equinovalgus) nor flexion (calcaneo-valgus), should we desire to weaken eversion without abolishing it, the principle on comparative lines would be to divide the stronger and selected everter (peroneus brevis), and to retain the weaker everter (peroneus longus). 


\section{THE MUSCLES OF THE TOES (GREAT SCIATIC NERVE)}

THESE may be considered under two divisions :

(I) Those which move the hallux or big toe.

(2) Those which move the four lesser toes.

\section{MUSCLES ACTING ON THE HALLUX}

These are :

(a) Two extensors.-Extensor longus hallucis and extensor brevis digitorum (innermost tendon).

(b) Two flexors.-Flexor longus hallucis and flexor brevis hallucis.

(c) Abductor hallucis.

(d) Adductor hallucis.

Extensor Longus Hallucis.-This slender muscle arises from the middle two fourths of the front of the fibula and the adjacent interosseous membrane, and its tendon, after crossing the front of the ankle, is inserted into the base of the distal phalanx of the great toe.

Extensor Brevis Digitorum.-This arises on the dorsum of the foot from the front part of the superior surface of the os calcis, and from the outer or lateral extremity of the anterior annular ligament. Passing forward on the dorsum of the foot it gives rise to four muscular bellies, the two inner of which are the larger. These bellies terminate in four tendons. The innermost tendon (extensor brevis hallucis) is inserted into the dorsal surface of the base of 
the proximal phalanx of the great toe. The remaining tendons pass to the adjacent three lesser toes. In monotremata (platypus and echidna) this muscle mainly arises from the fibula; but with higher mammalian development it gradually recedes from the leg to the foot, and the peroneus tertius represents, as stated, the remains of its fibula origin.

The long extender (extensor longus hallucis) has its fulcrum at the interphalangeal joint of the big toe. It extends the distal phalanx of the hallux. It does not flex at the ankle joint, as is commonly taught. By its

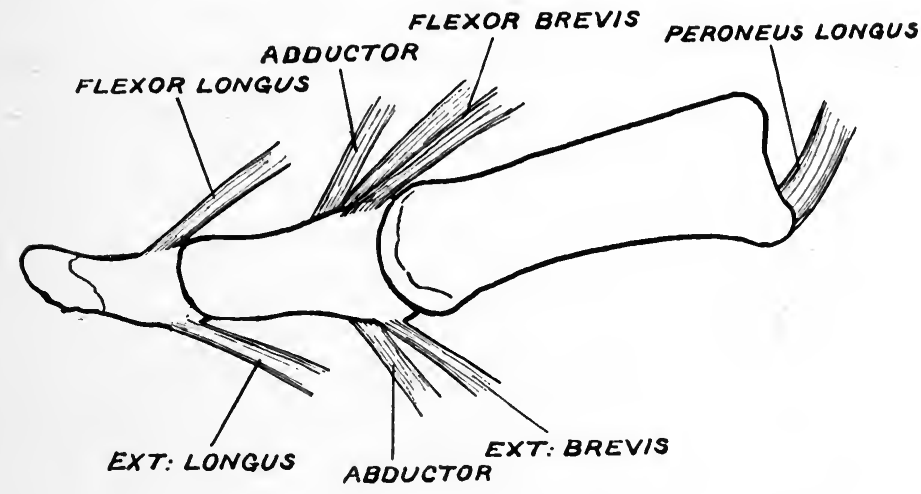

Fig. 86.-The muscles of the big toe (hallux).

action the extensors of the ankle are not called into a state of relaxation. This can only happen when the flexors of the ankle (tibialis anticus, peroneus brevis, and peroneus tertius) contract. The short extensor has its fulcrum at the metatarso-phalangeal joint. It produces extension there, acting on the proximal phalanx. It is interesting, then, to note that the big toe has two separate extending muscles. In children with talipes varus (inversion of the foot) division of the tibialis anticus is usually required. Its proximity to the extensor longus hallucis should be remembered, as the two are sometimes divided in a subcutaneous tenotomy, with subsequent "dropped toe" deformity. The operation in children 
should always be an open one, and the extensor hallucis should be identified and hooked out of the way.

It is worth remembering that the extensor longus hallucis and extensor longus digitorum are held down by the annular ligaments or straps, so as to follow the contour of the ankle and dorsum of the foot. They do not take the shortest course to the toes. If they did, just as in the case of the biceps brachii without the bicipital fascia, this would result in prominence and so in liability to injury. Although the short course would have resulted in improvement of strength of action, velocity of action would be diminished, and the toes rendered less elastic and more rigid. If tendons were not firmly held in relation to bones, but were allowed to rise during muscular action, the effects of such action would be to a large extent negatived.

Flexor Longus Hallucis.-This muscle arises at the back of the leg from the lower two thirds of the posterior or dorsal surface of the fibula. Its tendon passes in grooves on the back of the lower end of the tibia-on the back of the astragalus-and on the under surface of the sustenaculum of the calcaneus. It then enters the sole of the foot, where it is displayed in the dissection of the second layer of muscles, and is finally inserted into the base of the distal phalanx of the great toe.

Flexor Brevis Hallucis.-This muscle is placed on the inner side of the sole of the foot, corresponding to the metatarsal bone of the hallux, and is demonstrated in the dissection of the third layer of muscles on the sole. It arises by a pointed tendon from the inner side of the cuboid bone and the tendinous insertion of the tibialis posticus. Passing forward, its muscular belly separates into two portions, each of which terminates in a tendon which is inserted on either side of the base of the proximal phalanx of the big toe. On each tendon a sesamoid bone is developed. The inner tendon blends with the abductor hallucis and the outer with the adductor hallucis. The long flexor is the bender at the interphalangeal joint of the big toe, acting on the distal phalanx. It is the antagonist of the long extensor. The 
short flexor produces bending at the metacarpo-phalangeal joint, acting on the proximal phalanx. It is the antagonist of the short extensor.

One cannot fail to admire the means adopted by nature to effect leverage so as to improve the action of the flexors of the big toe. In each tendon of the flexor brevis hallucis a sesamoid bone is developed, by means of which, as in the case of the quadriceps and the patella, the power of the muscle is improved. But, in addition, as the muscular belly of the flexor brevis separates into two portions, a canal is formed by the two heads along which the flexor longus passes to the distal phalanx, and the tendons of each belly, supported by the sesamoids, thus form a pulley for the free action of the long flexor. The great use of the flexor longus hallucis is to keep the big toe in contact with the ground when we walk, being an important factor in raising the body on the great toe. This is especially seen after accidents dividing the tendon, when, with the big toe extended from over-action of the long extensor, we lose that "springiness" on the foot so important in the propulsion of the body.

Abductor Hallucis. - This is found amongst the first layer of muscles on the sole. Its bony origin is from the inner knob on the under surface of the calcaneus. Its muscular belly terminates in the tendon which is inserted into the inner or medial side of the base of the proximal phalanx of the big toe.

Adductor Hallucis.- This consists of two portions, an oblique and a transverse portion. These are demonstrated in a dissection of the third layer of muscles on the sole. The oblique portion arises from the sheath of the peroneus longus tendon and the bases or tarsal extremities of the third and fourth metatarsal bones. The transverse portion is narrow, and is placed transversely across the distal or phalangeal extremities of the metatarsal bones. This portion arises from the plantar ligaments of the three outer toes. The two portions, together with the outer tendon of the flexor brevis hallucis, blend at their termination and are 
inserted into the outer side of the base of the proximal phalanx of the big toe. The transverse portion of the adductor hallucis was wrongly described by the older anatomists under the name of transversus pedis. It was regarded as arising from the big toe, and as being inserted into the fore part of the metatarsus of the little toe so as to act as an adductor minimi digiti. As the little toe, which is struggling hard to retain its place in the human foot, has an adducting interosseous, it is difficult to understand why the adducting power of the little toe should be strengthened in preference to that of the "selected" toe or hallucis. A study of the adductor transversus in the gorilla shows that this is one of the short muscles giving grasping power to the hind thumb or hallux.

The adductor hallucis draws the big toe outwards towards the lesser toes. The abductor hallucis draws the big toe inwards away from the lesser toes. The plane for abduction and adduction corresponds to the axis of the second toe. The transverse part of the adductor, by its contraction, draws not only the big toe towards the lesser toes, but approximates also the heads of the metatarsal bones so as to increase the curve of the transverse arch of the metatarsus. This denotes that origin and insertion of muscle approximate. There is no necessity, however, for hollowing or "guttering" of the foot, and this transverse portion should be looked on, like the oblique portion, as a pure adductor of the hallux. Apart from this action the front of the foot is strengthened by the support given by a transverse band of muscle, with the power of contraction and relaxation in preference to the less perfect support of a transverse ligament.

As there is not the freedom of lateral movement in the great or lesser toes to the same extent as in the fingers, we are apt to think that abduction and adduction are of little importance. Interference with the lateral movement of the big toe, however, is the cause of what is perhaps the most common factor interfering with gait in modern life, namely bunion of the big toe, which is associated with the 
pathological condition known as hallux valgus. This I have invariably found associated with flat foot. In hallux valgus the head of the metatarsal bone of the big toe projects on the inside of the foot, and the phalangeal segment is directed outwards towards the other toes. An angular deformity results, and over the projection a bursa develops, which usually becomes thickened and inflamed. This condition is more common in women than in men, and no doubt is largely dependent on the narrow boots usually worn interfering with the spread of the foot. The condition is aggravated by high heels throwing the body weight on the ball of the great toe.

In treatment it is the muscular factor we have to consider, just as in flat foot. No one presumably regards yielding of plantar ligaments as the cause of flat foot. The essential factor in flat foot is muscular-strong everters and weakened inverters. Similarly, in hallux valgus the abductor hallucis as the result of pressure is in a weakened state, analogous to a paralysed muscle-its action for years having been interfered with-and the result is over-action of the adductor hallucis. No doubt this weakness of the abductor hallucis is a secondary contributing factor towards the condition of flat foot. In treatment the foot should be kept constantly at a right angle in a splint, and the big toe held forcibly abducted, so as to stretch the contracted adductor hallucis, and take the strain off the abductor to allow it possibly to recover. No effect can be obtained in less than eight weeks, and the splint should be worn every night for a considerable period afterwards, daily attempts at re-education of the abductor being made. When walking is permitted the boot should be arched on the inside, and the sole and heel raised along the inner edge, so as to take all strain off the ball of the toe.

The boot' should be broad anteriorly to allow of spread for the toes, and is best at first laced to toe, so that when the boot is put on the big toe can be abducted by wool before lacing. In old severe cases operation, such as excision of the metatarso-phalangeal joint, may be neces- 
sary. Since the hallix is the selected toe care should be taken by the surgeon to avoid if possible its removal in cases of accident. Should this be necessary, the power not only of the foot but of the leg for walking and standing is greatly impaired.

\section{MUSCLES ACTING ON THE FOUR LESSER TOES}

These are:

(I) Extensors.-Extensor longus digitorum and extensor brevis digitorum.

(2) Flexors.-Flexor longus digitorum, flexor accessorius, flexor brevis digitorum, flexor brevis minimi digiti, and lumbricales.

(3) Abductors and adductors.-Abductor minimi digiti and dorsal and plantar interossei.

\section{(I) Extensors}

Extensor Longus Digitorum arises from the upper three fourths of the anterior surface of the fibula and from the outer tuberosity of the tibia. Its tendon passes in front of the ankle and divides into four separate slips which pass to the four lesser toes. On the dorsum of the foot these tendons pass obliquely from within outwards.

Extensor Brevis Digitorum.-This arises on the dorsum of the foot, from the front part of the superior surface of the calcaneus and from the outer extremity of the anterior annular ligament. Passing forward on the dorsum of the foot it gives rise to four muscular bellies, the two inner of which are the larger. These bellies, which pass obliquely from without inwards, terminate in four tendons. The innermost passes as described to the great toe, and the remaining three pass to the second, third, and fourth toes, lying on the outer side of the long tendons.

The tendons of the long and short extensors are distinct on the dorsum of the proximal phalanges of the second, third, and fourth toes, becoming associated at the distal extremity. They are firmly connected to the proximal phalanx. The combined tendon is traced over the middle 
to the distal phalanx, being connected with both. In the case of the little toe, which appears to be struggling hard to retain its place in the foot, the short tendon is absent, and extension is produced by the long extender only. These muscles extend the phalanges of the second, third, fourth, and fifth toes, and it would seem from special dissections I have made in reference to this question, that motor power for extension of the middle and distal phalanges is largely derived from the short extender. The oblique direction of the short extending tendons counteracts the opposite obliquity of the long extenders, so that when the two act together-and their action is inseparable-we have an even extension of the toes. Man not being provided like the ape with an opposable hallux, there is not the same freedom of the toes as of the fingers, nor the same fine co-ordinations.

\section{(2) Flexors}

Flexor Longus Digitorum (Perforans).-This arises from the middle three fifths of the dorsum of the tibia, and its tendon passes behind the inner malleolus and enters the sole of the foot, where it is demonstrated in a dissection of the second layer of muscles. In the sole it is joined by the flexor accessorius, and, dividing into four tendons for the second, third, fourth, and fifth toes, each is inserted into the base of the distal phalanx.

Flexor Accessorius.- This small fleshy muscle lies on the sole of the foot, and arises from the lower part of the calcaneus by two divisions, which unite and are inserted into the tendon of the flexor longus digitorum before its division. The pull of the flexor longus is obliquely from within outwards. By means of this accessory muscle, not only is the action of the flexor longus strengthened, but its pull on the toes is made in a directly backward direction instead of inwards. It is interesting to note that Cowper considered the accessorius as part of the lumbricales. It was his opinion that, just as the long flexor divided into four tendons, so also did the accessorius divide into the fleshy muscles or lumbricales. 
Flexor Brevis Digitorum(Perforatus). -This forms the middle of the first layer of the muscles on the sole of the foot. It arises from the plantar fascia and the inner or medial tubercle of the calcaneus, and its muscular belly divides into four tendons for the four lesser toes. Each tendon at the base of the proximal phalanx divides into two to allow of the passage of the long tendon-these two portions then unite to form a grooved channel for the long tendon-and they finally separate and are inserted on each side of the middle phalanx. This arrangement of the long and short flexors is analogous to that of the profundus and sublimis on the fingers, and by this arrangement space is economized and a double system of leverage improvised, as explained in the description of the finger tendons.

Flexor Brevis Minimi Digiti.-This arises at the base of the metatarsal bone of the little toe and the sheath of the peroneus longus as it crosses the sole of the foot, and is inserted into the base of the proximal phalanx of the little toe internal to the abductor. This muscle assists in bending the little toe at the metacarpo-phalangeal articulation.

Lumbricales. - These are four in number, and arise in the sole of the foot from the tendons of the flexor longus digitorum. Each lumbrical ends in a tendon which is traced forward on the inner side of the four lesser toes to the inner dorsal portion of the base of each proximal phalanx. There is a fibrous connection on the dorsum of the proximal phalanges between the interossei, lumbricales, and extensor tendons. As regards the action of the flexors of the toes, the lumbricales by acting on the proximal phalanges, bend at the metatarso-phalangeal joints of the four lesser toes. The flexor brevis bends at the first interphalangeal joint owing to its attachment to each middle phalanx, and the flexor longus bends at the second interphalangeal joint owing to its attachment to the distal phalanx. The flexor longus, although it arises in the leg, does not extend at the ankle joint so as to assist the gastrocnemius and soleus in walking. It can act in either the position of flexion or extension of that joint. By curving into the sole of the 
foot round the inner malleolus improved leverage is effected, - the malleolus acting as a pulley for the play of the tendon. Although the obliquity of the pull of the long flexor is counteracted mainly by the accessorius, the short flexor is also a counteracting force against the oblique pull, since it arises from the calcaneus and its tendons rather incline obliquely inwards. These muscles assist walking by keeping the toes in relation to the ground.

\section{(3) Abductors and Adductors}

Abductor Minimi Digiti.-This is seen in the first layer of muscles on the sole of the foot on the outer or lateral side. It arises from the under surface of the calcaneus, and is inserted into the outer side of the base of the proximal phalanx of the little toe external to the flexor brevis minimi digiti. Its action is to draw the little toe outwards from the others, resembling the function of a dorsal interosseus.

Interossei. (a) Dorsal.-These are four in number and arise by two heads from the adjacent sides of the metatarsal bones. Each muscle terminates in a tendon which is traced to the outer side of the bases of the proximal phalanx. The first two pass to either side of the second toe, and the remaining two to the outer or lateral side of the third and fourth toes. The big and little toes have their own special abductors.

(b) Plantar.-These are three in number and, arising from the corresponding metatarsal bones, pass to the inner side of the bases of the proximal phalanges of the third, fourth, and fifth toes. The big toe has its special adductor, and the second toe has a dorsal interosseus on either side.

In the foot the imaginary line for the lateral action of the toes passes through the axis of the second toe. In the hand the line of action is to or from an imaginary line through the axis of the middle finger. Owing to the diffe ences in the adjustments and utility of the hand and the foot the interossei in the foot of man have not the importance they have in the hand. The dorsal interossei inserted into 
the outer sides draw the toes from the imaginary line through the second toe. The two dorsal interossei for the second toe oppose each other and abduct separately to one or other side. When not separately in action they maintain an equilibrium as regards lateral motion in this toe, and this applies equally to the opposing adductors and abductors in the other four toes. The plantar, inserted on the inner side, adduct or draw the third, fourth, and fifth toes towards the second. The little toe is abducted by the abductor minimi digiti, and the big toe is abducted from the second toe by the abductor hallucis and drawn to it by the adductor hallucis.

\section{Rest and Re-education for Muscles acting on the Foot and Toes (Sciatic Nerve Paralysis)}

There is not the same specialization of function in the human foot as compared with the hand. The opposability of the hallux to the toes has been lost, and instead of a mechanism like the hand, which might almost be described as "cerebral," the foot has become merely a mechanical support for the body. A person who has lost the foot may with artificial support walk so well that the defect is undetected. In the upper limb, not merely the loss of the hand, but even of one muscle, such as the pronator teres or the opponens pollicis, may be sufficient to ruin the utility of the limb. Furthermore, we find that owing to the differences in the control of the nerves of the hand as compared with the foot, we do not obtain the same response to rest and re-education in the latter as in the former. In treatment an essential point is to maintain the foot at a right angle with the leg. By this I do not mean a forced dorsi-flexion, which is less than a right angle and an uneasy position.

The foot of a patient which is in the position of "calcaneus" (acute flexion at the ankle) or "equinus" (acute extension at the ankle) is in a position of comparative inutility. By maintaining a position of equilibrium at the 
ankle as regards flexion and extension we prevent overaction of either the inverters or everters, thus preventing the development of varus (inversion) or valgus (eversion). The most common cause of "paralysis" in the leg and foot muscles is infantile paralysis. Trauma, gunshot wound or other injury of the great sciatic or peroneal (external popliteal) nerves, are also not uncommon causes. What we are apt to forget is that the injury of the great sciatic nerve in the thigh means an impairment of function on both the dorsal and plantar surfaces of the foot. Rarely in cases of gunshot wounds of the back of the thigh do we meet with interference with the action of the hamstrings. The sartorius and gracilis are not, we must remember, supplied by the great sciatic, though they act as benders at the knee.

Should the peroneal (external popliteal) nerve alone be involved there is interference with function of the muscles of the front and outer aspect of the leg. The following movements are lost : extension of the toes (long and short extenders); flexion of the ankle (tibialis anticus, peroneus longus, and peroneus brevis) ; inversion of the foot in the flexed position (tibialis anticus); eversion of the foot in either the position of flexion or extension (peroneus brevis and peroneus longus). In a case of recent shell wound of the outer part of the upper leg, which had involved the peroneal nerve at its division into the anterior tibial and musculo-cutaneous branches, the patient when seen had foot-drop, with a contraction of the tendo achillis and slight tendency to inversion (equino-varus). He was unable to evert in either the position of flexion or extension at the ankle, owing to weakness of both the peroneus brevis and peroneus longus. He was unable to invert in the flexed position at the ankle owing to a paralysed tibialis anticus ; but inverted freely in the extended position owing to free action of the tibialis posticus supplied by the posterior tibial nerve (fig. 87). Such a foot should be immediately rested in a splint with the foot-piece at a right angle, and the back-piece should extend to the middle of the thigh 
(fig. 93), so as to stretch not only the tendo achillis at the ankle, but the gastrocnemius and plantaris at the knee. The foot should be placed midway between inversion and eversion, and the toes kept hyper-extended by means of wool. Should, in such cases, contraction of the tendo

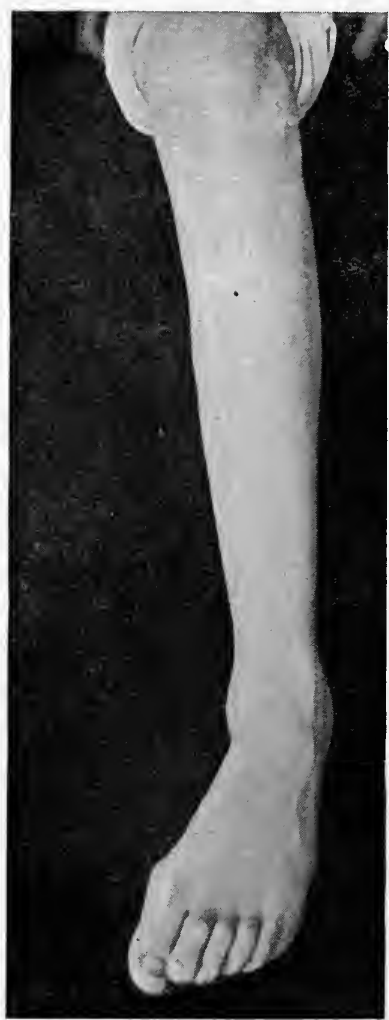

FIG. 87.-Inversion of the foot in the extended position (tibialis posticus).

achillis and tibialis posticus be severe, lengthening of the tendons may be necessary in order to remove any mechanical interference with recovery.

The patient should be encouraged to make frequent voluntary attempts at extending the toes and flexing the ankle while in the splint. The foot should also be lifted 
out of the splint in the position it occupied when at rest, the heel being rested on the palm of the attendant's left hand and slight flexion of the toes and extension at the ankle allowed. The patient is then encouraged by voluntary effort to regain the position of rest-the amount of work being gradually increased. Testing for minimum function and commencement of re-education should be carried out with the patient lying well on the opposite side, the lower limb being rotated so that the dorsal surface of the affected foot looks downward and the plantar surface upward. This position can be initially obtained with the patient lying on his face, and the feet hanging over the end of the bed-the ankles being meanwhile supported on a pillow. With the ankle extended (plantar flexion) we obtain an improved leverage for the weakened muscles, in addition to the benefit of gravitational effect. The patient is gradually, with recovery, brought to the dorsal position.

Should the internal popliteal nerve be involved alone, we find the foot in the position of dorsi-flexion at the ankle, owing to paralysis of the muscles acting through the tendo achillis and of the tibialis posticus. There is also inability to invert the foot in the extended position (tibialis posticus). Flexion and lateral movements of the toes are also lost. In this condition the foot should be rested on a back splint and foot-piece. The foot-piece should be extended so as to allow the foot to be in the position of equinus, and its extremity bent to allow flexion of the toes. The backpiece should extend to the middle of the thigh and be bent behind the knee to prevent straining of the gastrocnemius. Re-education should be on lines opposite to that for the peroneal nerve, work being commenced with the patient on the back and the heel dependent. In a lesion of the great sciatic nerve involving all the muscles of the front and back of the leg and foot, the foot is best rested in the position given above for lesion of the peroneal (external popliteal) nerve.

As regards prognosis and treatment in this condition I 
may mention the case of a boy who was aged $I I \frac{1}{2}$ years when seen, and was suffering from "paralysis" of the right foot. The cause was infantile paralysis. The leg and foot were thin and cold, and the boy, when walking, swung the foot so as to avoid the toes dragging on the ground. No movements of the toes, nor motion at the ankle of either flexion or extension could be detected. The foot, owing to gravity effect, was in the position of equinus. $\mathrm{He}$ certainly seemed a fit subject for one of the numerous surgical procedures advised for flail ankle. Treatment by various measures had been carried out continuously for about five years. The foot was placed in a splint with the foot-piece at right angles, the back of the splint reaching to the upper third of the leg. In this way gravitational effect was overcome. With the heel supported when taken out of the splint, the boy, who for three months was kept in bed, was encouraged many times daily to endeavour to flex or extend the toes and foot, attention being paid particularly to the flexors of the ankle.

Though at first this was without apparent result, movement gradually returned in the toes, and in six months a definite tendo achillis could be felt. The extensor action was more powerful than the flexor, and although the footdrop had lessened there was still little power over flexion at the ankle in spite of twelve months' relaxation. The deep fascia over the long extensors of the toes and of the tibialis anticus was removed by an incision over the front of the leg. By this means room was given to these muscles for expansion. The fascia, to which they are closely attached, is frequently found contracted down on them when they are paralysed. As the result of its unyielding character it allows no room for their expansion when recovery of the muscles is taking place. This procedure was followed by immediate improvement in this case, so that in six days the patient was not only able to flex and extend the toes, but to flex the ankle almost fully as well. A soldier who had his great sciatic nerve completely severed (and subsequently repaired) in the thigh by a shell wound 
was seen six months afterwards. With three months of similar treatment, including division of the fascia, though there was no movement in either foot or ankle, there was no deformity present, and the patient was able, when lying down, to maintain the foot at a right angle when it was lifted off the bed, and could walk without splint support.

In the foot, then, the important thing is the prevention of deformity, the ideal being a foot at a right angle. When surgical procedures are necessary, such as division or lengthening of tendons or tendon transplantation, we have to consider in connection with lateral movements of the foot that there is inversion and eversion in both the flexed and in the extended position of the foot. Also that the two inverters and the two everters produce their own particular action only when the foot is in a state of equilibrium as regards flexion and extension.

It is in connection with the latter position that the question of muscular selection comes into consideration. If we should desire to weaken inversion or eversion which muscle tendon should be divided? Numerous types of splints have been devised for the foot; they are only scientific when they are dominated by principles of muscle rest and muscular action. Where we wish to keep the foot at a right angle it should be firmly held at the ankle; otherwise in a few hours after the application of the splint, the heel will be found well away from the foot-piece, frequently with a lateral deviation as well.

The following is a useful form of foot splint which can be applied for equinus, valgus, or varus, and the foot-piece can be extended for calcaneus. The splint is made of tin in two pieces, soldered together at the heel-one for the foot, and one for the leg-the latter extending to the junction of the middle and upper third. For the avoidance of pressure and to allow for the play of an ankle strap the leg-piece is opened at the heel (fig. 88). The sole of the foot not merely rests against the foot-piece, but the edges of the latter are turned up for I inch on the inside and $\frac{3}{4}$ inch on the outside, 
thus forming lateral shields to keep the foot in position. The splint is lined with blanket and covered with chamois. The extension to the middle of the thigh (fig. 93, p. 224) is made by means of a stem of malleable iron $\frac{5}{8} \times \frac{1}{8}$ inch. Two wings are attached to this by means of rivets-one in the

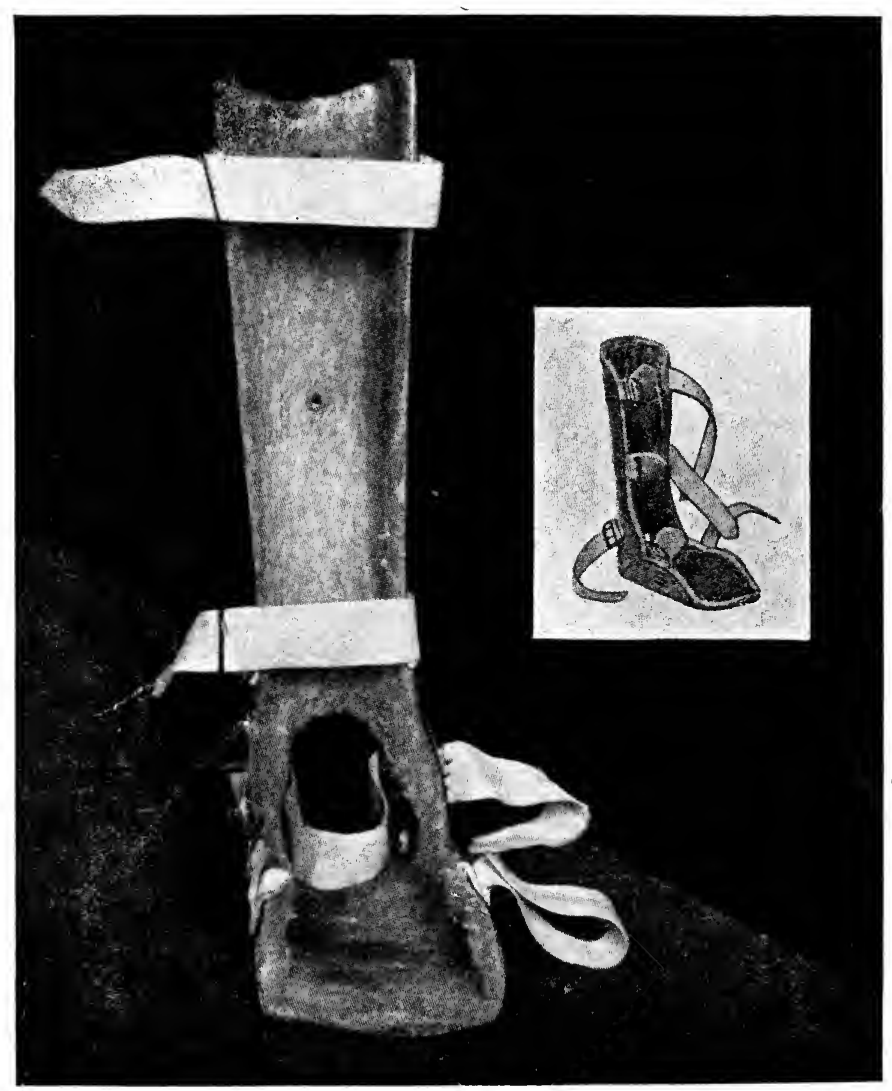

FIG. 88.-A useful form of foot splint. The arc shoe.

vicinity of the knee joint and one at the upper extremity in the thigh. The splint is attached by means of buckles and webbing straps. The ankle strap, in a case of inversion of the foot, is attached to the lower end of the leg-piece on the outer side. By means of the opening in 
the back of the lower end of the leg-piece it is brought up on the outer side of the patient's ankle, across the front of the ankle, and descends on its inner side, and passing out through the opening is attached to a buckle on the inner side of the lower end of the leg-piece. By means of this attachment a constant pressure is exerted on the outside of the foot so as to favour eversion. The reverse attachments would be made in a case of eversion of the foot. 


\section{CHAPTER XI}

\section{MUSCLES OF THE SPINE -}

ARISING from the back there are certain muscles concerned with the motion of the upper extremity with whose movements they have been described. They are inserted into the humerus or scapula, being the trapezius, latissimus dorsi, rhomboids, and levator anguli scapulæ. There are also muscles arising from the back that are inserted into the ribs. These are concerned with respiration. They include serratus posticus superior, serratus posticus inferior, and levatores costarum, and will be described under the mechanism of respiration. The muscles of the back may be conveniently considered under three groups, viz. :

(I) Those which move the head.

(2) Those which move the neck.

(3) Those which move the back and loins (thoracic and lumbar spines).

\section{MUSCLES WHICH MOVE THE HEAD}

In connection with the movements of the head we have to consider two sets of articulations.

\section{(I) Articulation of the Atlas with the Axis}

We have the odontoid process of the axis forming an articulation above with the anterior arch of the atlas, and below with the transverse ligament on which it is supported. There are also joints on either side between the articulatory surfaces of each bone. As the articulation of the occiput 
with the atlas is practically a pure hinge joint, it is between the atlas and axis that rotatory movements are performed - the atlas, carrying the head with it, moving upon the tooth-like or dentate process of the axis. The odontoid process, by means of which rotation of the head is rendered possible, represents the separated body of the atlas united with that of the axis. It is found persisting as an independent bony piece in reptilia, for example, lizards, and also in platypus.

\section{(2) Articulation of the Atlas with the Occiput}

These two bones are connected by antero-posterior and lateral occipito-atlantal ligaments. The two upper articulatory surfaces of the atlas are somewhat oval, and hollowed to receive the condyles of the occipital bone. By means of the obliquity of each component forming the articulation rotatory movements are prevented, and although slight lateral movement is permitted, it is here that flexion and extension of the head or the forward and backward nodding movements take place. The axis is also directly connected to the occiput by the occipito-axial ligament and the odontoid or check ligaments. The atlas was so called owing to the fact that it supports the globe or cranium, and the axis owing to the tooth-like (dentate) process on which the atlas turns.

The movements of the head on the spine are :

(I) Flexion and extension (bending or nodding forwards, and bending or nodding backwards), with slight lateral movement to one or other side, taking place between the head and the atlas.

(2) Rotatory movements. These are performed by the atlas together with the head moving on the odontoid or tooth-like process of the axis as on a pivot.

The muscles are divided into two main groups-postvertebral-dorsal, or extensors, and pre-vertebral-ventral, or flexors, 
(I) Post-Vertebral, or Extending Muscles

There are on each side :

Splenius capitis.

Complexus.

Trachelo-mastoid.

Rectus posticus major.

Rectus posticus minor.

Superior oblique.

Inferior oblique.

The last four muscles form the boundaries of the suboccipital triangle.

Splenius Capitis.-The splenius was so named because it lies like a surgical splint on each side of the neck. Though the splenius capitis and splenius colli are usually described together owing to their similar origin, they are two distinct muscles, capable of separate dissection, and the capitis lies more ventral than the colli. No less an authority than Albinus made the distinction. He regarded splenius capitis as that part arising from the spines of the vertebræ of the neck and inserted into the cranium, and splenius colli as that part arising from the dorsal or thoracic vertebræ and inserted into the cervical vertebræ. This muscle arises from the lower half of the ligamentum nuchæ and spinous process of the last cervical and upper five or six dorsal vertebræ. It is inserted into the mastoid process of the temporal bone, thus obtaining a grip well forward on the skull, and also into the lateral part of the superior curved line of the occipital bone. When both these muscles (splenii capitis) act, they pull the head directly backwards on the atlas-extend the head; when one acts it pulls the head obliquely backwards to one side.

Complexus.- This muscle has been so called owing to the complexity of its fleshy and tendinous parts. It arises by a series of tendons from the lower four cervical and the upper six or seven dorsal or thoracic vertebræ. The thick fleshy belly, partly tendinous and partly muscular, filling up the hollow at the sides of the cervical vertebræ is inserted 


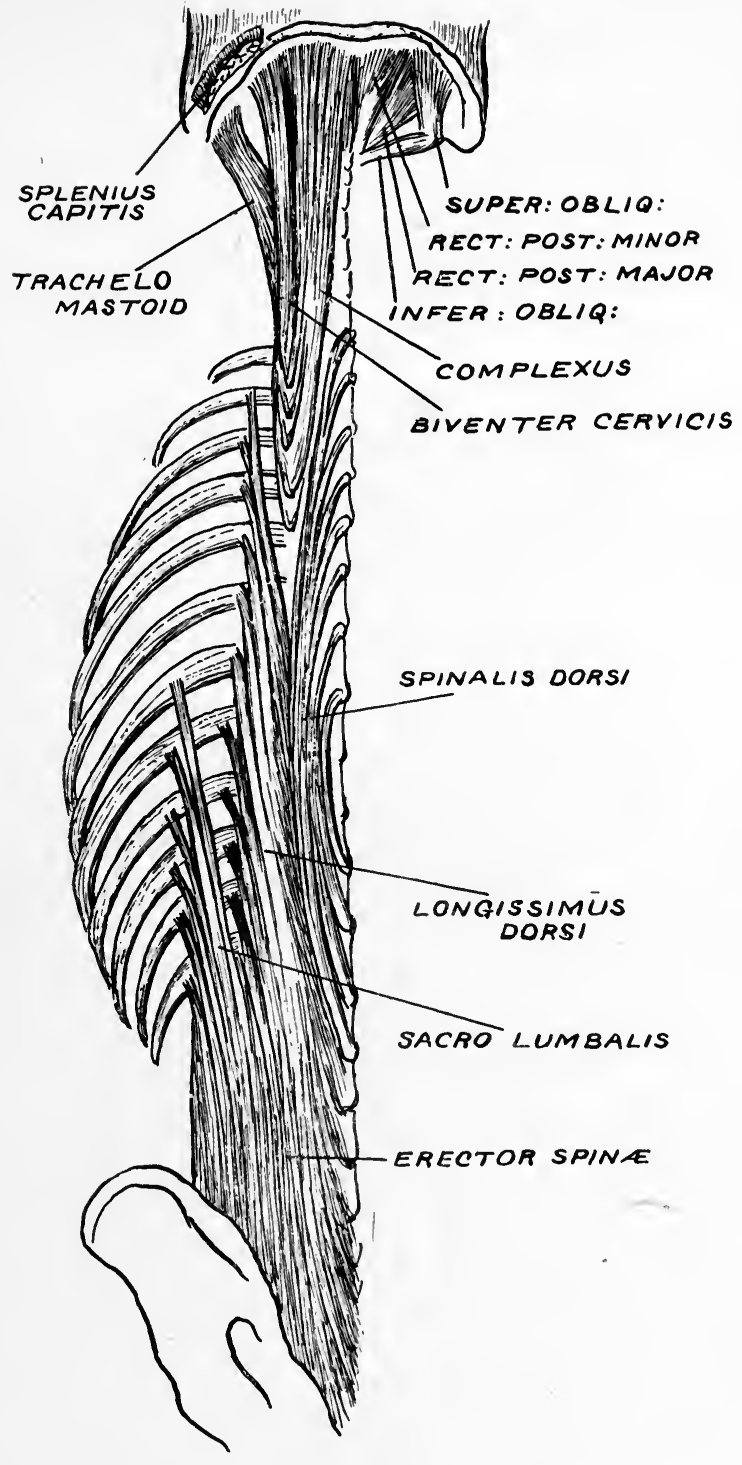

FIG. 89.-The extenders (dorsi flexors) of the head. The erector spinæ and its divisions. 


\section{I}

".

- . . 
into the occipital bone between the superior and inferior curved lines. Albinus regarded the inner part of the muscle, which is fleshy above and below and tendinous centrally, as a distinct portion, under the name biventer cervicis. It is best to regard it, however, as the inner part of the complexus. When both complexi act they extend the head, draw it backwards and so assist the splenii ; when one acts the head is drawn obliquely backwards, to one side. Thus, both the splenii and complexi are important factors in maintaining the head erect.

Trachelo-Mastoid.-This small muscle, so called owing to its origin in the neck and insertion into the mastoid, has also, owing to the mixture of tendon and muscle in its belly, been called the complexus minor. It arises by separate tendons from the lower cervical and six upper thoracic vertebræ, and is inserted into the mastoid process of the temporal bone beneath the splenius. Its use is like that of the complexus and splenius. When one acts the head is drawn backwards obliquely to one side; when both act the head is extended or drawn directly backwards.

Rectus Posticus Major.-This muscle passes upwards and outwards on each side from the spine of the axis to the inferior curved line of the occipital bone and adjacent surface of bone below it.

Rectus Posticus Minor.-This muscle passes on each side from the tubercle or spine of the atlas to the occipital bone below the inferior curved line, and internal to the preceding. When the muscles of the two sides act the recti postici pull the head directly backwards (extend) on the atlas; when one acts it draws the head obliquely backwards to its own side. Older anatomists called the smaller posterior recti, passing from the atlas to the head, the renuentes or backward nodders, in opposition to the small anterior recti, which were called annuentes or forward nodders of the head.

Superior Oblique.- This arises from the upper surface of the transverse process of the atlas, and passing upwards and inwards is inserted into the occipital bone between the two 
curved lines. In spite of their name these muscles do not, as might be imagined, rotate the head on the atlas. This movement is not permitted at this articulation. Acting together these muscles draw the head directly backward on the atlas; acting singly the head is drawn obliquely to one or other side.

Inferior Oblique.-This muscle, passing between two vertebræ, is not a direct muscle acting on the head, although through its action rotation of the head is possible. It passes outwards on each side, from the spine of the axis to the projecting transverse process of the atlas, by means of which great leverage power is obtained. When the muscle of one side acts, the other-its opponent-is in a state of relaxation and elongation. The result of contraction is to rotate or roll the atlas round the odontoid process of the axis, so that the transverse process of the atlas is moved towards the spine of the axis. As apart from ligaments there is a direct muscular connection between the atlas and cranium, it follows that the head, whether in the state of flexion on the atlas or extension, is also rotated with the atlas on the odontoid process of the axis, and the face turned to the same side.

\section{(2) Pre-Vertebral or Bending Muscles}

These are on each side (fig. 90).

Rectus capitis anticus major, or longus capitis.

Rectus capitis anticus minor.

Rectus lateralis.

Sterno-mastoid.

Rectus Capitis Anticus Major.-This arises from the front of the transverse processes of the third, fourth, fifth, and sixth cervical vertebræ, and is inserted on each side into the basilar process of the occipital bone.

Rectus Capitis Anticus Minor.-This small muscle lies beneath the preceding, and passes from the atlas to the basilar process of the occipital bone, dorsal to the preceding.

Rectus Lateralis.-This muscle arises from the trans- 
verse process of the atlas, and is inserted into the jugular process of the occipital bone. These muscles are the benders of the head on the atlas, flexing the head directly forwards when both sides act, or bending it obliquely to one side when only one muscle acts. They may be desig. nated flexores capitis.

Sterno-Mastoid.-This muscle, passing obliquely across the side of the neck, is probably the most prominent in the body. It arises at the lower part of the neck from the first part of the sternum and inner third of the clavicle, and is inserted above into the mastoid process of the tem-. poral bone and the superior curved line of the occipital bone. When both sterno-mastoids act they flex the head on the atlas, and by their aid the chin can be brought down to the chest. Their action is especially seen when we raise the head from the body in the recumbent position. When only one sterno-mastoid acts it bends the head obliquely downwards towards the shoulder of the same side. This muscle, when it contracts, does not rotate the head so as to carry the face towards the opposite side. Its fulcrum or centre of motion is the articulation between the occiput and the uppes surface of the atlas, and rotation does not take place at this articulation. The movements of flexion and extension at the articulation between the atlas and the occiput represent a striking example of the first order of leverage. Thus, in backward movement of the head, the dorsal or extending muscles represent the power, the front of the head represents the weight to be raised, and between the power and weight is the fulcrum, or centre of motion, at the occipito-atlantal articulation. 


\section{CHAPTER XII}

\section{MUSCLES WHICH MOVE THE NECK}

WhILE the preceding muscles move the head together with the atlas or first vertebra only, the remaining vertebræ of the neck remaining unmoved, the following muscles act directly on the vertebræ of the neck, and so move the head indirectly. The former are the proper muscles of the head, and the latter the common. Flexion and extension movements are free in the cervical region. These movements are also free in the lumbar region; but while extension is most free in the cervical, flexion is most free in the lower lumbar region. In addition, lateral movements and slight rotation are permitted. Speaking generally, the greatest mobility of the spine is in the cervical region. This is allowable owing to the thickness of the fibro-cartilage permitting of a large amount of elasticity, small size of the vertebræ, horizontal position of the short spinous processes, and the oblique direction of the articulations. The muscles acting on the cervical vertebræ may be divided into two main groups :

(I) Dorsal-post-vertebral-or extensors.

(2) Ventral-pre-vertebral-or flexors.

(I) Dorsal-Post-Vertebral-or Extending MUSClES

These are :

Splenius cervicis or colli.

Costalis cervicis (cervicalis ascendens, ilio-costalis cervicis).

Transversalis cervicis (longissimus cervicis). 
These latter muscles have been regarded as continuations of the main divisions of the erector spinæ, and named accordingly. It would be better to dissociate them from the muscles of the loins, as they are distinct muscles with distinct functions.

In addition to these three muscles we have in the cervical region-as in the dorsal and lumbar regions-innumerable and intricate small muscles filling up the hollows and interstices on the back of the spine on each side. These, from their dispositions, are called semi-spinales, multifidus spinæ, inter-transversales, and inter-spinales.

Splenius Cervicus. - This arises on each side, in common with the splenius capitis, from the ligamentum nuchæ and the spinous processes of the last cervical and upper six dorsal vertebræ, and is inserted into the transverse processes of the three upper cervical vertebræ. When both these muscles act they draw directly backwards or extend the upper cervical vertebræ ; when only one acts the upper vertebræ are drawn obliquely backwards to one side, by which movement the head is directed towards the opposite shoulder.

Costalis Cervicis.-This arises on each side from the angles of the upper six ribs, and is inserted by a series of narrow tendons into the transverse processes of the fourth, fifth, and sixth cervical vertebræ.

Transversalis Cervicis. - This arises on each side from the transverse processes of the upper six dorsal or thoracic vertebræ, and is inserted into the transverse processes of second, third, fourth, fifth, and sixth cervical vertebræ.

These two muscles are, like the preceding, extensors of the cervical vertebræ, helping to keep the neck erect. If either acts alone the cervical vertebræ are drawn obliquely backwards.

Semi-spinales. - These partly tendinous muscles, one set on each side, were so called as belonging half to the transverse and half to the spinous processes of the vertebræ. They are met with in connection with the movement of both the dorsal and cervical spine. Those for the move- 
ment of the cervical vertebræ arise on each side from the transverse processes of the upper six thoracic (dorsal) vertebræ, and are inserted into the spinous processes of all the cervical vertebræ except the atlas. When the muscles of the two sides act they draw the cervical vertebræ, except the atlas, directly backwards, and so help to extend the neck. When those of one side act only, they undoubtedly, from the obliquity of their direction, cause rotation of the cervical vertebræ and indirectly of the head. By means of the inferior oblique muscles, which pull in an opposite direction, the short and rapid turnings of the head are probably carried out.

Multifidus Spinæ.-Unlike the semi-spinales these muscles are more marked below than above. The multifidus lies along the hollow of the back on each side of the spinous processes, and is found in the loins, back, and cervical region. It was split up formerly by anatomists into numerous divisions, but these are so connected that they have been described under a single name, "multifidus spinæ." In the cervical region the muscular bundles are inserted into the spines of all the vertebræ except the atlas: those for the lower cervical vertebræ arising from the transverse processes of the upper thoracic vertebræ, and those for the upper cervical vertebræ arising from the articular processes of the four lower cervical vertebræ. In the neck the multifidus fibres resemble in action those of the semi-spinales. When those of the two sides act they draw the cervical vertebræ, except the atlas, directly backwards, and so help to extend the neck; when those of one side act they cause rotation of the cervical vertebræ, and so indirectly of the head.

Inter-transversales.-These small slips extend between the transverse processes of the spine. They are best developed in the cervical region, where they are double, owing to the fact that lateral motion in the neck is possible, and the transverse processes forked. They extend from the atlas (between it and the axis) to the seventh cervical, and the bundles are most numerous where movement is 
most marked, namely between the atlas and axis. When the muscles of one side act, these fibres, by approximating the transverse processes, produce some lateral movement in the cervical vertebræ, bending them to the one side; when both act they help to maintain the erect position of the neck.

Inter-spinales.-These are short muscular bundles, most marked in the cervical region where movement is best, extending between the spinous processes of the vertebræ. They were first discovered by Cowper in I69o. When they contract they draw the spines of the vertebræ of the neck nearer, and so help to extend the cervical spine.

(2) Ventral-Pre-Vertebral-or Flexing MusCles

They are :

Longus colli.
Three scaleni: anticus, medius, and posticus. $\}^{\text {See fig. } 90^{\circ}}$ p. 2 Io.

Longus Colli.-This long flat muscle, which lies on each side on the front of the cervical vertebræ, extends from within the thorax below to the atlas above. Three parts are recognized: Superior oblique portion passing from the transverse processes of the third, fourth, and fifth cervical vertebræ upwards and inwards to the tubercle on the front of the atlas. Vertical portion passing from the front of the bodies of the upper three thoracic and lower three cervical vertebræ to the front of the second, third, and fourth cervical vertebræ. Inferior oblique portion passing up and out from the upper three thoracic vertebræ to the transverse processes of the fifth and sixth cervical vertebræ. By means of the division of this muscle into three portions added strength is given to its contraction. When it acts with its fellow of the opposite side it bends the cervical spine forwards, and so indirectly the head; when the muscle of one side acts the cervical spine is bent laterally to one side.

Scaleni.-These muscles were so called owing to their resemblance collectively on each side to a scalene triangle. 
The true anatomy is to regard the scalenus anticus, medius, and posticus as one flat triangular muscle extending from

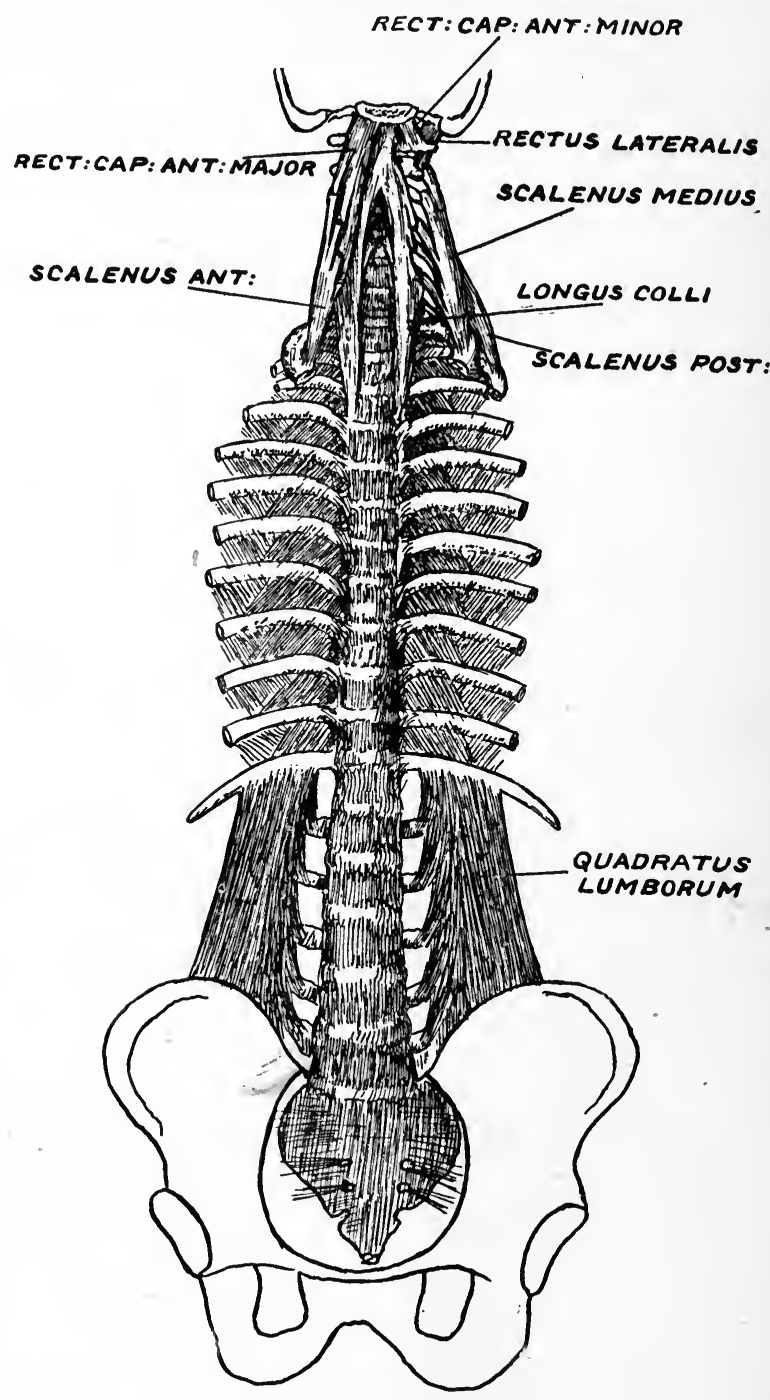

FIG. 90.-The benders (with gravity) of the head and spine.

the first and second ribs below to the transverse processes of the six lower cervical vertebræ above. It is usually, 
however, described in three portions on each side, though Winslow described but two, Douglas four portions, and Albinus no less than five portions.

The Anticus passes from the scalene tubercle on the inner part of the upper surface of the first rib near the cartilage, almost vertically upwards to the transverse processes of the third, fourth, fifth, and sixth cervical vertebræ; the Middle passes between the upper surface of the first rib dorsal to the anticus and the transverse processes of the lower six cervical vertebræ; and the Posticus passes between the upper edge of the second rib near the spine to the transverse processes of the lower three cervical vertebræ.

As regards the action of the scaleni muscles, discussion is always raised as to whether they are flexors of the neck primarily and muscles of inspiration secondarily, or inspiratory primarily and flexors secondarily. It seems to be generally agreed that when the fixed point is below these muscles flex the cervical spine, and when the fixed point is above they elevate the first two ribs and are muscles of inspiration. Of one thing, however, we can be certain : they are either muscles of respiration or flexors of the cervical spine, but they are not both. We must refer the fulcrum or centre of motion either to the ribs or to the vertebræ, not to both. The scalenes have not two separate fulcra or centres for action.

We would first observe that though there are powerful muscles on the back of neck acting as cervical extenders, yet antagonizing these so far we have only described one muscle, the longus colli. At once it may be argued that the action of the longus colli is favoured by gravity ; in other words, that an effort is required to hold the neck extended and the head erect. If we are recumbent, however, something more powerful is needed to flex the extended neck than the longus colli. Again, should the origin of these muscles be at the spine, there would be no particular reason for the multiform origin from all the cervical vertebræ except the atlas, in order to secure an action 
on the first two ribs. On the other hand, acting from below, a strong grip is secured by this multiple attachment in flexing the cervical spine. Furthermore, the attachment to elastic ribs-elastic owing to the interposition of cartilage between them and the sternum-would rather strengthen than weaken muscular pull on the vertebræ.

The scaleni, acting together, bend the cervical spine directly forwards, assisting the longus colli. When those of one side only act the cervical spine is bent to one or the other side. In this action they are more powerful than the muscles longus colli, though in the former action the longus colli, from their median position, are the more powerful. Should the fixed point for the action of these muscles be above, they would flex the trunk at the cervico-dorsal junction of the spine. This requires much practice, though I have frequently seen it performed by acrobats and jugglers when lying on the back with the head and neck fixed.

As regards lateral movement of the cervical spine, and indirectly of the head, this is performed by the combined action of the muscles of one or other side-the actions of the two groups on the front and back being, as regards flexion and extension of the neck, in a state of equilibrium : the cervical spine is neither dorsi-flexed nor ventro-flexed. In this position lateral movement is best performed. Should we, however, dorsi-flex (hyper-extend) or ventro-flex (hyperflex) lateral movement is greatly impaired, especially in the former position, although rotatory movements can be made. In other words, with hyper-flexion or hyper-extension one set of muscles is contracted, and the other relaxed and elongated. In the mid state, or state of equilibrium as regards flexion and extension, contraction of one side with lateral movement is possible, owing to relaxation and elongation of those of the opposite side. 


\section{CHAPTER XIII}

\section{MUSCLES WHICH MOVE THE BACK AND LOINS (THORACIC AND LUMBAR REGIONS)}

IN the thoracic (dorsal) region of the spine flexion and extension reach their minimum especially in the upper half, and in the middle of the back scarcely any motion is permitted at all. In the lumbar region these movements are free, especially in the lower portion. Flexion movement in the spine is probably greatest between the fourth and fifth lumbar vertebræ. The small mobility in the dorsal region is associated with the vertical direction of the articular processes, the small amount of inter-vertebral substance compared with the lumbar region, and the over-lapping of the spinous processes.

As a result the thoracic spine becomes a more or less fixed point for the attachment of muscles acting on the neck as well as for the action of the intercostal muscles, since the centre of motion of the ribs is at the spine. In the thoracic region, in the upper half rotation can take place, but not in the lower part. Rotation is absent in the lumbar region, but here lateral motion can take place. The motion between individual vertebræ is limited, but in the whole spine collectively it becomes a very appreciable amount. By means of the curves in the cervical, thoracic, lumbar, and sacral regions, the spine is enabled to yield to pressure in the direction of these curves, and so to break shocks in violent motions of the body; this would be impossible were the spine a perpendicular structure, by which its elasticity would be lost. This elasticity is also effected by means of the spinal column consisting of a number of small bones instead 
of one unyielding bone for the protection of the spinal cord. Not merely must the cord be protected, but the spine must accommodate itself by its flexibility to the motions of the body, which it is enabled to do by means of a multiplicity of joints.

The muscles in this region may be primarily divided, as in the cervical region, into two divisions, posterior or dorso-vertebral for extension, and anterior or pre-vertebral for flexion.

\section{Dorso-Vertebral or Extending Muscles}

These are on each side

Erector spinæ and its three divisions:

Ilio-costalis.

Longissimus dorsi.

Spinalis dorsi.

In addition, as in the cervical region, we have innumerable and intricate small muscles filling up the hollows and interstices on the back of the spine on each side. These, from their disposition, are called semi-spinales, multifidus spinæ, rotatores, inter-transversales, and inter-spinales.

Erector Spinæ.-This is the mass, partly muscular and partly tendinous, placed at the root of the spine dorsally on each side. It is attached to the back of the crest of the ilium, posterior sacro-iliac ligament, back of the sacrum, and the spines of the lumbar vertebræ. From this muscle three columns arise, outer or ilio-costalis, middle or longissimus dorsi, and inner or spinalis dorsi (fig. 89, p. 202).

Ilio-Costalis.-This passes up and outwards on the back of the chest wall and is inserted into the angles of the lower six ribs. This small muscle, little more than a muscular ligament (accessorius or ilio-costalis dorsi), passing from the lower six to the upper six ribs would appear in man to be little more than an ancestral relic.

Longissimus Dorsi.--This is the main division of the erector spinæ, and has an attachment to the transverse 
process of the lumbar vertebræ. It has a double set of insertions, viz. to the transverse process of the thoracic vertebræ and also into the back of all the ribs, so that it has an extensive grip for extension.

Spinalis Dorsi.-This is so called because it is entirely attached to spinous processes. It does not pass to the neck. It arises from the spines of the lumbar and lower two thoracic or dorsal vertebræ and is inserted into the spines of the upper thoracic vertebræ.

Semi-spinalis.- So called on account of belonging partly to transverse and partly to spinous processes of the vertebræ ; it passes on each side from the transverse processes of the lower six thoracic vertebræ to the spinous processes of the four upper ones.

Multifidus Spinæ.-These are not only present in the cervical region as described, but in the thoracic, lumbar, and sacral regions as well. In the lumbar regions they arise from the articular processes and in the thoracic region from the transverse processes, and are inserted into the spinous processes of the vertebræ above, some of the fibres passing between adjacent vertebræ, others to second, third, or fourth vertebræ above.

Inter-spinales.-These short muscles bundles between the spinous processes of adjacent vertebræ are, as stated, well developed in the cervical region, are almost entirely wanting in the thoracic spine, where the vertebræ are almost fixed except at upper and lower parts, and are found again in the lumbar region.

Rotatores Spinæ.-These are found only in connection with the thoracic (dorsal) spine, and extend on each side between the transverse process of one vertebra and the lamina of the vertebra above.

Inter-transversales. - These extend between adjacent transverse processes of the vertebræ. Like the interspinales they are almost absent in the thoracic spine, except below and above, but in the lumbar region they are well developed for the lateral or twisting movements of this part. 


\section{Action of the Dorsal Muscles}

The Erector Spinæ and its divisions act as extenders of the spine, serving to maintain the spine erect and so counteracting the tendency of the body to fall forwards. Its power is increased owing to the extensive arborisations of

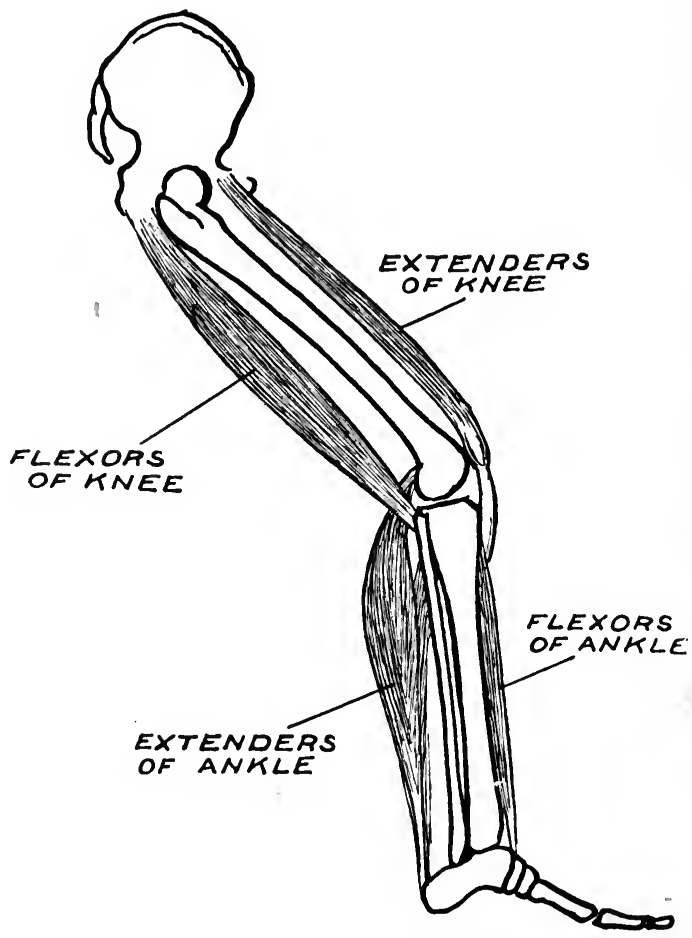

FIG. 91.-Flexion of the rigid body at the knee joint.

its insertions. As stated, there is a comparative absence of forward or backward movement of the thoracic spine, and the chief movement is in the lumbar joints, and especially at the junction of the thoracic and lumbar curves of the spine. When the muscles of the one side act the spine is drawn backwards to one or other side. An extreme instance of the action of the erectores spinæ is seen in acrobats, who, standing erect, are enabled to throw their 
heads backwards and drink from a glass of water placed on the floor. Here we have at first a condition of hyperextension (dorsi-flexion) of the head and at the loins, and, with the spine thus fixed, the flexors of the knee, with the legs and feet as fixed points, flex the trunk and thigh on the leg at the knee joint (fig. 9I). I have never seen this done as a pure back extension movement, and I doubt whether such a movement is possible.

The Semi-spinales are not found in the lumbar region. When the muscles of one side act they cause, owing to the obliquity of their direction, rotation of the thoracic vertebræ ; and as these muscles act similarly on the cervical spines the body is turned to the opposite side. Acting together they help to support the spine against gravity.

The Multifidus Spinæ. - In the thoracic region both sets, acting together, help to prevent the spine being bent forward by gravity. When the muscles of one side act rotation of the vertebræ is produced and the trunk is turned to the opposite side.

In the lumbar region extension is produced when the muscles of the two sides act, and lateral movement when the muscles of one side only act.

The Inter-spinales approximate the spines of the lumbar vertebræ, and so help extension.

Rotatores. - These assist the multifidus and semi-spinales muscles of the same side, so as to rotate the dorsal vertebræ and turn the trunk to the opposite side.

The Inter-transversales are well developed in the lumbar region, and, by approximating the transverse processes, produce the lateral or twisting movement which is found in this portion of the spine.

\section{Anterior or Pre-Vertebral Muscle (Quadratus LUMBORUM)}

It is interesting to note that there is no pre-vertebral muscle in the thoracic region of the spine, where flexion and extension are practically absent. Extending force in that region must be exerted chiefly against gravity. 
Quadratus Lumborum.-This muscle, called quadratus owing to its somewhat square shape, forms part of the dorsal wall of the abdomen, and extends between the iliac crest and the last rib (fig. 90, p. 210). It arises below from the dorsal part of the crest of the ilium for about two inches, and from the ligaments connecting the back of the ilium to the sacrum and to the lumbar vertebræ. As it ascends ventral to the lumbar vertebræ it sends tendinous slips into the transverse processes of the four upper ones. In addition, it secures an attachment to the lower edge of the last rib, and a small process is traced beneath the arch of the diaphragm, to be inserted into the body or ventral part of the last thoracic vertebra. When the muscles of both sides act they flex the spine at the lumbar vertebræ, and so are the physiological antagonists of the erectores spinæ; when the muscle of one side acts the spine is bent towards the same side. When the psoas minor, which extends from the side of the first lumbar vertebræ to the ilio-pectineal line of the pelvis, is present, it would act as a flexor and assist the quadratus lumborum.

Should the dorsal and cervical spines be fixed, as when, for example, an acrobat lies on his back, contraction of the quadratus results in flexion of the sacrum, pelvis, and so of the lower limbs at the lumbar joints. Contraction of the erectores spinæ, when an acrobat is lying prone, will similarly produce extension at these joints. Thus, the quadratus is an important factor in maintaining spinal equilibrium. We cannot help being struck by the paucity of muscular engines on the front of the spine for the purposes of flexion, compared with the multiplicity of muscles which subserve the function of extension, i.e. erection of the head, neck, and back. Apart from neutralizing their antagonists these dorsal muscles are constantly fighting gravity when we either stand or sit. These many and strong muscles are important factors in preventing the body from falling forwards, to which there is a continual liability - a liability increased by the projection forwards from the spine of the head as well as the thorax. When 
we stand motionless these muscles are, we must remember, actively contracted. There is no such state as skeletal balance apart from muscular action.

\section{Pelvic Tilting}

As stated, lateral movement is free at the lumbar region; when standing we can roll the trunk from one side to the other on the pelvis and lower limbs, so as to bring the lumbar vertebræ nearer to- the os ilium. Just as in the cervical region, when this motion is permitted, the muscles producing flexion and extension at this region are in a state of equilibrium as regards their own particular movements. The contraction of both groups of one side, alternately, results then in lateral rolling. If we flex at the lumbar region or extend, the pre-vertebral and post-vertebral muscles are in a state of alternate relaxation and contraction, and lateral movement is not permissible, as we can readily prove on ourselves. When we lie down with the spine fixed and the pelvis and the lower limbs free, this lateral action, i.e. the combined action of the pre-vertebral and postvertebral muscles on one side raises the pelvis and lower limb on the same side, and depresses them on the other. This action causes the so-called "pelvic tilt." In this position the normal relationship of the anterior superior spines to the medial abdominal plane or to the umbilicus is upset, producing apparent lengthening and apparent shortening of the lower limbs. This is independent, as seen in cases of hip disease, of the position assumed at the hip joint, which is dependent solely on the action of the muscles whose fulcrum or centre of motion is at that articulation.

\section{SPINAL REST}

Weakness of the neck muscles (paralysis), with inability to hold the head erect, is not frequent in infantile paralysis ; in a series of Ioo cases it was only met with twice-and in both instances it was associated with paralysis of all four limbs and inability to sit up. These cases do well, owing, I 
apprehend, to the fleshy character of the cervical muscles and to the good natural rest recumbency affords-maintenance of which alone, apart from the encouragement of voluntary muscular action, usually suffices for recovery. " Paralysis," of the back muscles, associated with inability to maintain the erect position when sitting in bed, is more frequently met with than cervical affection, and usually accompanies, in poliomyelitis, affection of all four limbs and frequently paralysis of the two lower ones. Complete rest of the trunk and lower limbs in a double Thomas's splint, and with an extension to the head to prevent dorsi-flexion, if neck affection be present, is advisable. With effective rest at the outset good recovery with absence of deformity is the rule.

In the absence of effective rest, pelvic tilting or spinal curvature, or both, are serious possibilities. There is little doubt that the majority of cases of severe lateral curvature are the result of neglected or inefficient rest treatment in cases of infantile paralysis. So important do I regard this, that in a case of upper limb paralysis, not only should the upper limb be rested in the abduction frame, but recumbency for a month should be insisted on, for the sake of convenience, in a double Thomas's hip splint. Only in this way can the danger of lateral deviation of the spine or rotation be anticipated. Not only is the treatment harmless, but from every point of view beneficial. Pelvic tilting from paralysis of the groups of one or other side, if not guarded against, occurs rapidly, and even in infants may resist all forcible measures for its correction. Its prevention is simple. It is associated with apparent lengthening or shortening of one limb or the other, and later, with the usual production of lateral curvature of the spine. Recumbency in a double Thomas's splint, with the lower limbs evenly abducted, will be sufficient to obviate this; or in the absence of cervical or upper limb affection, rest in a lower limb abduction frame, with equal abduction of the lower limbs, will suffice.

In fig. 92 is illustrated a girl aged three years, the victim 
of poliomyelitis. She was untreated by any form of splint, and the condition shown was present eight weeks after the onset. There is marked pelvic tilting, with apparent lengthening of the lower limb on the affected right side and shortening on the non-affected left side. There is

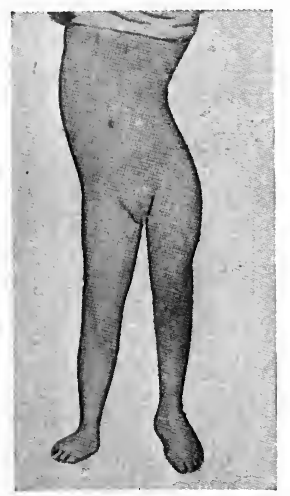

FIG. 92.-Pelvic tilting following poliomyelitis.

spinal curvature present, and the tilting did not respond to even forcible traction. In a case such as this the inclination is to raise the boot on the shortened, though sound, left pelvic side. The raising should be on the right, apparently lengthened, limb, so as to throw the pelvis as much as possible on to the left shortened one. Ultimately, in this neglected, avoidable, and unresponding condition the boot had to be raised on the shortened left side to enable the patient to ambulate. The pelvic tilting was due to a paresis affecting the quadratus lumborum and the erector spinæ on the right side, with uncontrolled action of the same muscles on the left side producing the tilt and shortening. 


\section{CHAPTER XIV}

\section{ANATOMICAL CONSIDERATIONS IN JOINT FIXATION}

\section{The Significance of Fixation and Cure}

THE use of joints in the economy is varied, and associated mainly with improvement of active and passive mobility, and hence of the utility of the otherwise rigid limb; also with the lessening of the risk of fractures and the dissipation of jars. Correlative and commensurate with the increased mobility of a joint, we find a multiplication and specialization of the muscles which act at it. Of the major joint functions-active and passive mobility and anti-concussion-active or muscular mobility is by far the most important. Hence we can say that morphologically and physiologically a joint is dominated by the muscles which act across it. For the muscle the joint exists, not the muscle for the joint. When we deal with the question of immobilization of a limb or part of a limb for the purpose of conferring " perfect rest" upon a damaged or diseased joint, the muscular factor should always be regarded as the important one. An endeavour must be made effectively and accurately to counteract the active and passive forces, which tend to alter that disposition of the component parts of the joint which has been selected by the surgeon. This disposition may be advised as being the most appropriate for securing ankylosis on the one hand, or for obtaining improved or normal range of movement on the other.

When we speak of perfect rest of a joint we refer of course to that position in which passive contact of 
damaged areas is reduced to a minimum by the proper disposition of the parts, and friction contact is eliminated by appropriate fixation. Counter-action of the passive forces - largely gravitational - can be easily and effectively accomplished. But when we come to consider the counteraction of the active forces operating against the maintenance of the position of " perfect rest," our attention must be paid to the origins and insertions of the muscles which act at the joint. The results aimed at in the treatment of diseased joints may be practically divided into two groups :

(I) Those in which recovery takes place with a complete or partial restoration of the articular function.

(2) Those in which subsequent mobility being hopeless, we endeavour to concentrate our attention on such fixation of the component elements of the joint as will be most utilitarian when ankylosis occurs.

As regards the first group, the affected joint is recovering when the range of motion of its component elements tends not to be diminished, but increased towards the normal. The progress of the cure is from the zero position of rest, along the lines of normal directions of motion, up to the limiting points of normal degree of motion along those lines; the attainment of this represents the perfection of cure.

When our ideal is adequate fixation, no subsequent alteration of the relation of the components forming a utilitarian ankylosis should eventuate. Regarded from the evolutionary standpoint, there is a much higher specialization and correlation with intellectuality in the upper than in the lower limb. It is more important to prevent ankylosis in the upper than in the lower extremity. The main function of the lower limb is the support of the body in the erect position; and in spite of ankylosis of the three major joints a patient may still be able to walk. In man, so even is the muscular adjustment in the upper limb that the loss of function of the adductor and opponens pollicis or of the pronator teres may be sufficient to ruin its usefulness. In the lower limb, 
since it is necessary to protect the joints from the shocks of locomotion and weight-bearing, recumbency should be insisted upon at all times in diseased or damaged conditions. As illustrations of these principles we may take the cases of the ankle and knee in the lower, and the wrist and elbow joints in the upper extremities.

Ankle Joint.-Here the position for rest and recovery, or for ankylosis, should be with the foot at a right angleover-action of the extensors, peronei, or tibials being associated with deformity, since it produces equinus, valgus, or varus. Owing to the position of origin of the ankle extenders forming the tendo achillis there can be no rest of ankle unless the power of action of these muscles is prevented. So-called immobility is usually secured by means of plaster of Paris applied round the joint, or by means of a splint passing to the upper third of the leg. These measures not only do not rest the knee joint, but are often ineffectual to prevent inversion or eversion of the foot or flexion of the toes.

These difficulties are overcome by means of the extended

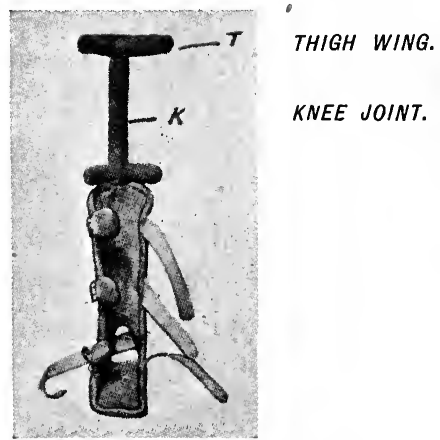

FIG. 93.-The extended arc splint.

arc splint (fig. 93) which passes above to the junction of the upper and middle thirds of the thigh, the construction of which has already been described (chap. v, p. I98). Should the ankle require dressing, such alteration may be made in the leg-piece as will permit of this without inter- 


\section{ANATOMICAL CONSIDERATIONS}

fering with the immobility of the joint. The position of the straps may be altered to suit any individual case.

Knee Joint.-The ideal position for rest or ankylosis is almost complete extension. Adequate rest can only be secured by such a position as secures immobility of both

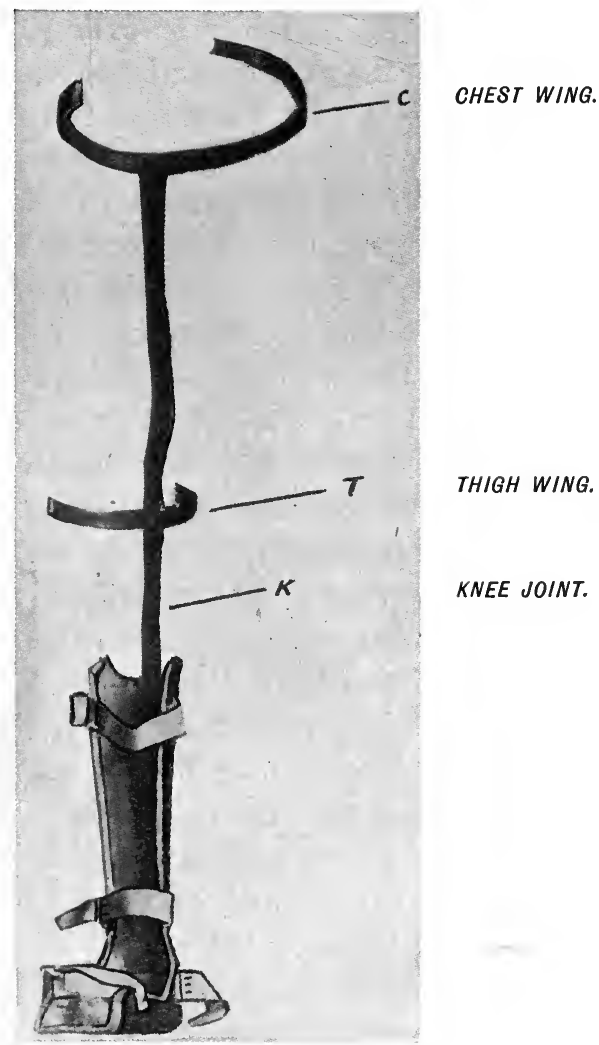

FIG. 94.-Effective splint for resting the knee joint.

the ankle and hip, owing to the positions of origin and insertion of the muscles about the knee. This may be obtained by means of a single Thomas's splint to which the ankle arc shoe is attached (fig. 94). If sinuses be present the wings may be altered to admit of dressing without interfering with the immobility of the limb. Under this 
treatment no contracture can occur, and the condition can be under daily observation.

The Thomas caliper splint, with the stems made of hollow tubular steel, is the ideal splint for use when the patient commences to ambulate. Plaster of Paris, extending from the mid thigh to the mid leg, is for many reasons absolutely worthless, and is the cause of so-called " rest treatment" being frequently followed by amputation.

Wrist Joint.-At this joint rest is usually secured by means of a flat wooden splint, or by means of a " cock-up" splint, which may be of metal, as in the Thomas hand splint which produces extension of the wrist and fingers, or of wood, as in Lister's splint in which the wrist is extended and fingers flexed over a cork piece-the limb being either

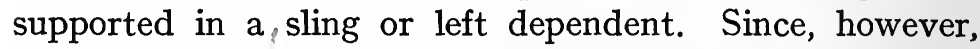
none of these control the elbow, and since flexors and extensors of the fingers and wrist arise above the elbow joint from the humerus, they cannot be regarded as perfect splints for securing strictly anatomical rest of the joint. For ankylosis, fixation in the extended position, owing to the improved leverage thus given to the flexors, admits of a strong grip; fixation in the flexed position of a weak grip. Furthermore, a wrist fixed in the position of overpronation is worthless, since flexion of the elbow would bring the back of the hand to the mouth. Similarly with fixation in the over-supinated position, in which food may be brought to the mouth on flexing the elbow, but the power of grasping the food is lost.

Elbow Joint.-Here the usual treatment is a rectangular splint or plaster of Paris-with the risk of ankylosis in a useless position, to say nothing of the interference with mobility if sinuses be present-or the position of acute flexion by means of a cervical slinger, the range of flexion being increased with recovery. By these methods neither the shoulder joint, through which the long head of the biceps courses, nor the wrist joint is rested, nor in the case of the latter appliance is the joint itself protected from local irritation. The ideal fixation would be rest in a position of 
flexion at about $45^{\circ}$, securing rest also for the shoulder and wrist-the angle of flexion as enunciated by Thomas being increased with recovery. If ankylosis be intended, this should be obtained in a position of flexion most suitable for the patient's trade or occupation.

In the upper extremity, so fine and correlated are the muscular adjustments that adequate fixation of one joint is best secured by fixation of all three, a point which never seems to be insisted upon. This may be simply obtained by means of the writer's upper limb abduction splint (fig. I8). By its means the shoulder is supported, and the elbow joint may be placed in any angle of flexion necessary. This can be altered with recovery or remain fixed at any angle for adequate ankylosis should this be the result desired. By means of the hand-piece the wrist and fingers may be extended and the hand placed midway between pronation and supination. In spite of the presence of sinuses which require dressing, the limb is always at rest whilst this is being done, and furthermore, the position of the wings may be altered for the individual case without interfering with its effectiveness. In altering the angle of flexion at the elbow joint with the limb supported by the abduction splint, we have this advantage over the position of acute flexion produced by a sling, that we are working with muscles whose origin and insertion are as nearly as possible on a level plane. It is worth remembering that in separation of the epiphysis of the humerus, where the treatment usually adopted is acute elbow flexion by means of adhesive strapping, the same position can be obtained by means of the abduction splint. This has the advantage, that the elbow, often greatly swollen, is supported, and alterations in the angle of the joint can be effected by means of a hinge in the splint without disturbing the fracture. Furthermore, the limb is not compressed, thus avoiding the liability to ischæmic "paralysis" of the forearm, of which unfortunately the strapping is a not infrequent cause. 


\section{CHAPTER XV \\ THE MUSCLES OF RESPIRATION}

THESE we may consider under three divisions, viz. :

(I) Muscles of the abdominal wall.

(2) Muscles of the diaphragm.

(3) Muscles of the thorax.

\section{Múscles of the Abdominal Wall}

These are five in number on each side, although the fifth pair is almost extinct in man. They are external oblique, internal oblique, transversalis, pyramidalis, rectus abdominis, and extend between the thorax and the pelvis.

External Oblique (Descendens).-This is the outermost and largest of the abdominal muscles, being muscular laterally and tendinous in front. Above, it is attached to the lower eight ribs by eight fleshy processes or digitations, and the muscular fibres pass obliquely downwards and forward. Dorsally, the muscle fibres from the lower ribs are attached to the anterior half of the crest of the ilium. The rest of the fleshy muscle, the middle and upper portions, terminates in an aponeurosis which, covering the whole of the front of the abdomen, joins with that of the opposite side at the mid line, the union being known as the linea alba; this extends from the sternum to the pubes. Below, the aponeurosis extends from the anterior superior spine of the ilium to the crest of the os pubis. This lower limit is the inguinal or Poupart's ligament. In the lower part of the aponeurosis, close to the spine of the pubis, lies the external abdominal or subcutaneous inguinal " ring." Formerly, many anatomists regarded the inguinal ligament as a 
distinct band into which the aponeurosis of the external oblique was inserted, and not as part of the aponeurosis itself. In general, however, in the mammalia below man, the external oblique has no attachment to the iliac crest, and the aponeurosis from the lower dorsal muscle fibres passes directly across the internal oblique to the external ring. Comparing this arrangement with Poupart's ligament in man, Arthur Keith regards it as one serving to strengthen the inguinal region, and so lessening the liability to hernia.

Internal Oblique (Ascendens).-This lies between the external oblique and transversalis, and is attached below to the outer half of the inguinal or Poupart's ligament, anterior two thirds of the crest of the ilium, and dorsally to the lumbar fascia. From this origin the fibres spread in a somewhat fan-shaped form. Behind they pass directly to the lower three ribs. In front they form an aponeurosis, which, at the linea semi-lunaris, splits to form with the aponeurosis of the external oblique and transversalis muscles the sheath of the rectus; and is finally inserted into the seventh, eighth, and ninth costal cartilages above and into the linea alba from the ensiform cartilage to the symphysis pubis. The fibres from Poupart's ligament pass downwards and inwards across the spermatic cord, and are inserted, by means of a tendon, with fibres of the transversalis into the pubic crest and ilio-pectineal line, forming the conjoined tendon.

Transversalis. - This is the inner of the flat muscles of the abdomen, and is so called on account of the direction of the fibres. Its origin below is from the outer third of Poupart's ligament, anterior two thirds of the iliac crest, behind from the lumbar fascia, and above from the inner surfaces of the cartilages of the lower six ribs. The fibres pass horizontally forwards, to terminate in an aponeurosis which is inserted into the linea alba, the upper three fourths passing behind the rectus and the lower fourth as in the case of the aponeurosis of the external and internal oblique, passing in front of the sheath of the rectus Some of the fibres of the aponeurosis 
below arch downwards, and are inserted with fibres of the internal oblique into the os pubis, forming the conjoined tendon.

Pyramidalis. - This small muscle may be wanting on one or both sides. It arises below from the pubic crest, and passing up and in terminates in the mid line at the linea alba, sometimes extending as far as midway between the pubes and umbilicus. This muscle has its greatest development in the monotremes and marsupials-animals "low" in the mammalian scale-and this is associated in both the male and female members of the two orders with the presence of the so-called epi-pubic or marsupial bone. These are two in number, one on each side, and extend for a varying distance up and out in the lower part of the anterior abdominal wall, articulating below with the horizontal ramus of the pubes. They are relatively larger in the monotremes than in the marsupials. In the former, descent of the testes has not taken place; but although in the marsupials the testes are still abdominal at the time of birth, yet they descend into the scrotum shortly after the young is transferred to the pouch, and occupy a prepenial and not a postpenial position. In the platypus the three abdominal muscles are to be distinguished, and the pyramidalis, or superior rectus, has an attachment on the inner side of the marsupial bone, and meeting its fellow at the linea alba may be traced as high as the sternum. The rectus abdominis, or deep rectus, is also attached to the marsupial bone and reaches as high as the first rib and coracoid bone. In the marsupial the lower fibres of the pyramidalis are transverse, below passing across the interval between the two marsupial bones, while above they are oblique in direction. The rectus, as in monotremes, may reach as high as the first rib. The marsupial or epi-pubic bone is represented in man by the internal pillar of the external abdominal ring, and according to Sir J. Bland-Sutton, “Gimbernat's ligament and the triangular fascia are remnants of the very stout ligament which, in marsupials, anchors the epi-pubic bone firmly to the ilio-pectineal line." 


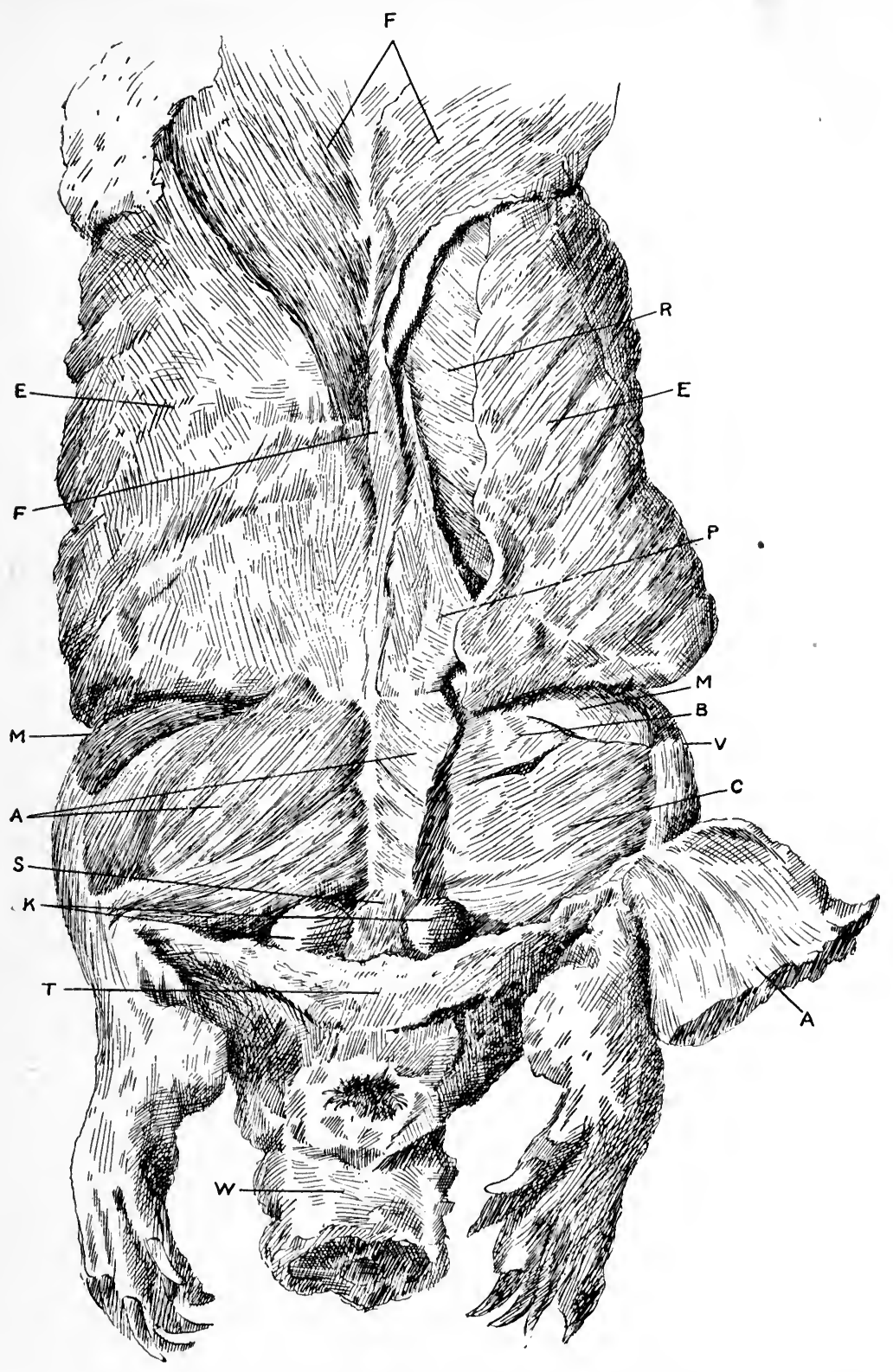

FIG. 95.-Dissection of ventral surface of the platypus (abdomen and thigh). F, Pectoralis major. R, Rectus abdominis. E, Ext. oblique. P, Pyramidalis (sup. rectus). M, Sartorius. B, Adductors. A, Gracilis. V, Tibialis anticus. C, I, eg flexors. $\mathbf{k}$, Cowper's glands. $s$, Fibres from gracilis to sphincter. T, Adductor intertibialis. w, Tail. 
Rectus.-This muscle extends along the front of the abdomen from the sternum to the pubes. In the mid line between the two muscles lies the linea alba, which is the meeting place of the aponeuroses of the oblique and transversalis muscles, and can be regarded as the fibrous continuation of the sternum to the pubes.

Below, each muscle is attached by two heads to the symphysis and crest of the os pubis. Above, it is inserted into the lower end of the sternum and fifth, sixth, and seventh costal cartilages. The sheath of the rectus consists in front of the aponeurosis of the external oblique and half the thickness of the internal oblique, and behind of the aponeurosis of the transversalis and the dorsal half of the internal oblique. This is only in the upper three fourths.

The lower fourth of the rectus has no dorsal sheath, since the aponeuroses of the abdominal muscles pass in front. Crossing the rectus are three to five tendinous transverse intersections-lineæ transversæ. These are not often found below the umbilicus, and are to be regarded as analogous to ribs which are developed in man in connection with the thoracic and not the abdominal muscles. Amongst reptilia, for example the crocodile, bony abdominal ribs are found. The lineæ semi-lunares are two curved tendinous lines, one on each side, corresponding to the outer edges of the rectus muscle. They are formed by the junction of the aponeuroses of the lateral muscles.

\section{Muscles of the Diaphragm}

Diaphragm. - This is a vaulted musculo-fibrous partition separating the cavity of the thorax from that of the abdomen. Its upper or thoracic surface is convex, and its lower or abdominal is concave. Its longest diameter is lateral and not dorso-ventral, the periphery of the diaphragm is muscular and the centre tendinous, being expanded or aponeurotic. Its periphery is attached to the whole of the inner circumference of the thorax, being attached in front to the ensiform cartilage of the sternum, laterally 
to the inner surface of the lower six ribs interdigitating with the transversalis. On account of this Bartholin regarded the diaphragm and the transversalis as but a single threebellied muscle, an opinion not far from the truth, since morphologically the triangularis sterni, transversalis abdominis, diaphragm, and levator ani are portions of one compressor sheet with the power of acting functionally together. Dorsally it is attached to the lumbar vertebræ by means of the two crura and the inner and outer arcuate ligaments. The crura are two muscular bundles, one on each side, attached to the upper lumbar vertebræ, and decussate before reaching the central tendon. The inner arcuate ligament on each side arches over the psoas, between the body and transverse process of the first lumbar vertebræ. The outer arcuate ligament arches on each side over the upper end of the quadratus lumborum, and passes from the transverse process of the first lumbar vertebra to the last rib. From the periphery the muscular fibres converge to a tri-lobed central tendon which lies nearer the ventral than the dorsal surface. The diaphragm is proportionately longest and most fleshy in the cetacea than in any other mammalian class, there being only a slight central tendon. The object of this is to allow the necessary distension of the lungs, which, as in the dugong, act as air bladders. Amongst certain of the ungulates also, in which we have movable ribs continued to near the pelvis, the diaphragm is proportionately large.

\section{Muscles OF THE Thorax}

The true muscles of the ribs are:

External intercostals.

Internal intercostals.

Serratus posticus superior.

Serratus posticus inferior.

Levatores costarum.

Triangularis sterni.

External Intercostals.-These are eleven on each side, 
occupying with the internal intercostals the spaces between the ribs. They pass obliquely downwards and forwards in a direction similar to the fibres of the external oblique of the abdomen, from the lower border of one rib to the upper border of the rib below.

Internal Intercostals. - These are eleven on each side, and their fibres are directed obliquely downwards and backwards like the internal oblique of the abdomen. They pass from the inner surface of each rib and the corresponding costal cartilage, and are inserted into the upper margin of the rib below. Passing in the same direction, but on a deeper level, are the Infracostales. These irregular bundles lie on the inner surface of the ribs. They may pass between three or more ribs near the angles, and are found especially in connection with the lower ribs.

Serratus Posticus Superior.-This lies flat at the upper and back part of the chest, and arises on each side from the spines of the last cervical and upper three thoracic vertebræ, and, passing down and outwards, is inserted into the second, third, fourth, and fifth ribs.

Serratus Posticus Inferior.-This is placed at the lower part of the back, and arises on each side from the spines of the last two thoracic and upper two lumbar vertebræ. Its fibres pass up and out, and are inserted into the four lower ribs.

Levatores Costarum or Supracostales.-These small muscular bundles, twelve on each side, might almost be regarded as portion of the external intercostals, just as the infracostales are of the internal intercostal. They lie close to the spine, and arise from the transverse processes of the last cervical and upper eleven thoracic vertebræ, and passing downward and outward are inserted each into the outer surface of the rib below them, close to the angle.

Triangularis Sterni.-This lies on the inner surface of the front of the chest. It arises from the side of the meso. sternum or manubrium and the inner surface of the lower sternum or ensiform, and its fibres pass up and out, to be inserted into the cartilages of all the ribs from the second 
to the sixth. The lower fibres are continuous with those of the transversalis abdominis.

\section{Action of Abdominal and Thoracic Muscles}

All these muscles, including the diaphragm, are essentially muscles of respiration. The principal muscle of inspiration -indeed of respiration generally-is the diaphragm, which was well-named " Nobilissimus post cor musculus." When it acts the thorax enlarges and air enters the lungs. The enlargement of the lungs is pari passu with the enlargement of the thorax, and any possibility that a vacuum would be formed is thereby obviated. Pressure is transmitted to the abdominal viscera, and in turn to the abdominal muscles, which being the antagonists of the diaphragm, are during its action relaxed and elongated. Distension of the air vesicles and the interchange of gases then constitutes the stimulus to action of the relaxed and stretched abdominal muscles. These contract, and not only pull down and compress the thorax, but react also on the abdominal viscera, with the result that the now relaxed diaphragm becomes in its turn elongated and restored to its normal state of upward convexity; and the thorax becomes diminished in diameter, as well as in the air-carrying capacity of the lungs. Such in brief is in my opinion the essence of the respiratory movements. These are carried out, not by the lungs, but by the muscles of the thorax and abdomen. In the case of the respiratory mechanism we have for consideration the action of muscles that are not only involuntary, but are also capable of being controlled by the will. Oxygen is as essential for the body when asleep as when awake. Voluntary action, on the other hand, is essential for the production of the voice, which depends on expiration; and it is also necessary in connection with the actions of the body as, for example, in violent exertions. It must be remembered that the greater the rigidity of the trunk the more effective will limb action become, and especially that of the upper extremity. When we propose 
a violent effort with this extremity we take advantage of a preliminary full sustained inspiration, dependent on the maximum effort of the muscles of inspiration, and in this way improve the resistance of the trunk.

\section{Function of the Diaphragm}

For the proper appreciation of the function of the diaphragm a reference must be made to its comparative anatomy. For an accurate knowledge of this we are indebted to Professor Arthur Keith, whose researches on this subject have placed the principles of the muscular mechanism of respiration on a sound anatomical basis. The great characteristic of the myology of the mammalia is seen in the development of a complete diaphragm separating thorax from abdomen. This is not more complete in man at one end of the scale than in the platypus at the other. With its presence there is associated a large increase in inspiratory power. It is customary to regard the diaphragm as having arisen gradually as a partition in the body cavity, shutting off the pulmonary from the abdominal viscera. There would seem to be little doubt however that in early vertebrate life the abdominal muscles, as well as the diaphragm, were at first concerned only with the circulation. With the introduction of ribs and lungs the abdominal wall, as well as the diaphragm, became respiratory. In fish and amphibians the septum transversum divides the body cavity into two portions-cardiac and abdominal; and in the latter in amphibians the lungs are situated. In reptiles the lungs are still abdominal. In birds the greater part of the lungs has become extruded from the abdominal cavity. From a study of the tailed amphibia the conclusion is reached that the mammalian diaphragm represents the cervical wall or diaphragm of the amphibian abdominal cavity, which became perforated by the development of the lungs. As Professor Keith puts it, "The lungs, like the testicles, may be regarded as organs extruded as herniæ from the abdominal cavity, because of certain physiological 
conditions that became of functional importance in the course of evolution."

The spinal part of the muscular diaphragm passes almost straight up to the central tendon, while the thoracic or sterno-costal portion passes up and backwards to this insertion also, and the diaphragm at rest is domed, the convexity being towards the thorax. With muscular contraction the central tendon descends, and by this means the capacity of the thorax is increased. It. was formerly held that the central leaflet descended least of all on account of its attachment to the pericardium, and the right leaflet less than the left on account of the liver. Hilton regarded the fibrous pericardium as the fascial insertion of the muscular diaphragm. The central tendon is intimately bound to the pericardium, and in all mammals the pericardium is strongly bound to the root of the lungs; it follows therefore that on inspiration the central tendon, pericardium, heart, and root of the lungs, formerly regarded as stationary, move downwards and forwards. It would appear however, from $\mathrm{X}$-ray studies, that the alteration of the diaphragm from the arched position to the horizontal is not so marked as was formerly supposed. The movement of the root is, according to Professor Keith, necessary for a proper expansion of the apical and dorsal part of the lung. The diaphragm is not a muscle acting on the lungs, but a muscle which increases the capacity of the chest by compressing the abdominal organs like a piston. This is rendered possible by the reciprocal relaxation and elongation of the abdominal muscles. While the relation of the diaphragm to the lungs varies, its relation to the liver and the abdominal contents is constant throughout the mammalia. In the monotremes and marsupials, especially the latter, there is a marked attachment between the liver and the diaphragm, and this is especially noticeable in the koala. To allow of the respiratory movements of the abdominal viscera, such as stomach, liver, kidneys, and spleen, we find that these organs are loosely fixed, moving downwards and forwards in response to diaphragmatic contraction. They are replaced by the 
contraction of the abdominal muscles during expiration, permitted owing to the reciprocal relaxation and elongation of the antagonistic diaphragm.

There is still much work to be done on the comparative anatomy of the diaphragm. Even John Hunter regarded fowls as possessing a thin transparent diaphragm running across the abdomen. In spite of the fact that there is a bilateral nerve supply, and that respiration is voluntary as well as involuntary, there is yet wanting any direct evidence that the right and left sides of the diaphragm have independent action. Embryology and experiments on the central nervous system tend to disprove the view of bilateral independence. A relative inequality in action between the right and left sides can be regarded as having a pathological and not a logical basis.

\section{Function of the Abdominal Muscles}

The abdominal muscles, which are essentially respiratory -being the direct antagonists of the diaphragm-should be regarded as continuous with the thoracic muscles (although ribs have developed in connection with the latter), of which morphologically they are a part. In reptiles, birds, and mammals we have the specialization of ribs and intercostal muscles for purposes of respiration. In fish the primitive arrangement of the muscles of the body wall which forms the basis of that seen in man, is a ventral longitudinal system and a lateral or oblique system. In the long-tailed amphibian three muscular layers are present in the body wall, of which the middle or internal oblique is the most important, as the transversalis and external oblique layers are derived from it during development. Within the middle or internal oblique layer the ribs are evolved. The intercostal muscles and the internal oblique of the abdomen are its representatives in man. Since in the amphibians the lungs are filled through the pharynx by the action of the muscles under the jaw, ribs and intercostal muscles as well as the diaphragm are primarily 
evolved, not in connection with respiration, but with circulation. They assist in regulating abdominal tension, and so the venous circulation. In a similar way abdominal ribs act, as is seen, for example, in the crocodile. In a transverse section of the thoracic wall of a reptile we find three layers: outer represented by the rectus and external oblique, inner by the transversalis, and a middle double layer represented by the internal and external intercostals which in the abdomen are combined as one layer, the internal oblique. From the primitive transversalis sheet is formed the diaphragm, triangularis sterni, transversalis abdominis, and the levator ani. With the evolution of lungs, and assisted by ribs which enabled the muscles not only to compress the body cavity but to allow of its expansion, the body musculature, though primarily circulatory in function, became respiratory. Further, with the evolution of the diaphragm as seen in mammals, and the extrusion of the lungs from the abdomen, the musculature of the body wall controlled not only abdominal but also thoracic pressure as well.

With the erect or orthograde posture of the anthropoid and man there was a further functional modification in the use of the abdominal muscles. The shape of the chest altered, the azygos lobe of the right lung between the heart and diaphragm, which is well developed in monotremes and marsupials, disappeared, and the heart came to rest on the diaphragm ; so that the abdominal muscles not only became respiratory, but had to support the abdominal organs as well. The abdominal muscles are the physiological antagonists of the diaphragm, not of the extensor muscles of the spine, hence they do not produce by their action flexion or rotation of the trunk. Contraction of the rectus does not call into physiological relaxation the erector spinæ, which has not the same centre of motion. In reference to the erroneous idea that the rectus abdominis is an important bender of the trunk when we stoop from the erect position to pick up an object from the ground, it is worth remembering that in the platypus-an aquatic 
mammal-the rectus is relatively better developed than in man and that a pyramidalis also is present, as well as an epi-pubic bone.

No doubt the peculiar arrangement of the abdominal muscles is for purposes of increased strength in relation to visceral support. The arrangement is such as to form, not a plane, but a curved surface, and of such a mechanical nature that, whilst the perpendicular pressure is a constant, the tangential pressure is a variable quantity. One could not imagine a better mechanical arrangement for the prevention of ventral hernia than that by which flat muscles, whose fibres run in different directions, are placed one on top of another. Elastic tissue alone instead of muscular for the abdominal wall would be useless however for the respiratory act, since it has not the power of contracting and producing motion.

We cannot regard the abdominal muscles as being anything more than passive agents in preventing the escape of the abdominal contents, but they are able to accommodate themselves, owing to their elasticity, to varying conditions of intra-abdominal pressure. The normal action of the uterus, urinary bladder, or rectum is independent of the action of the diaphragm or abdominal muscles. When the walls of the abdomen are weakened and ventral hernia develops-as from direct injury, poliomyelitis, or laceration, for example by a bullet, of the lower intercostal nervesthis resistance is weakened or lost. In cases of poliomyelitis affecting the abdominal muscles, rest in the supine position should be insisted on, preferably in a double Thomas's splint or a plaster of Paris casing. The erect posture should be attained slowly, the patient being gradually elevated in bed from the supine to the sitting-up position by means of pillows. Under this treatment, and owing also to the fleshy nature of the abdominal muscles, the prognosis of this comparatively rare condition is altogether favourable. Unfortunately it is frequently overlooked in its early stage, and the first evidence of its presence may be ballooning of one or other side of the abdomen. Hence 
the importance in poliomyelitis of insisting on recumbency at the outset. It is worthy of remembrance that when Cowper, more than two centuries ago, first observed that the lower fourth of the rectus abdominis was uncovered by tendon sheath-having only the transversalis fascia and peritoneum dorsally-he regarded it as an abnormality likely to lead to a weakening of the support of the body wall, " so that accident like this might be the cause of certain ruptures." In inflammatory conditions of the abdomen, such as appendicitis and acute peritonitis, the patient will endeavour to keep the parts as immobile as possible by bringing more into play the action of the thoracic muscles, and by moving the diaphragm in inspiration as little as possible. Diaphragmatic movement in these conditions means visceral movement, and visceral movement means pain. The abdominal muscles on one or both sides are kept in an apparent state of relative rigidity in these conditions, but they must relax, though to no great extent, for purposes of inspiration. Diminution of abdominal breathing is an important sign of serious abdominal mischief. It is on account of the reciprocal relaxation and elongation of the abdominal muscles during inspiration, that we ask a patient to draw a deep breath to enable the abdomen to be palpated thoroughly. An incision through the abdominal wall means an incision through respiratory muscles.

Professor Keith regards visceroptosis, or displacement of abdominal organs, as primarily due to failure in the supporting power of the abdominal muscles, and as a causative factor poliomyelitis must be remembered. Weakening of the abdominal muscles means less resistance to the diaphragm, which, by its contraction, forces downwards and forwards the abdominal contents. In true visceroptosis the diaphragm occupies permanently a lower or inspiratory position, and the abdominal organs are forcibly and permanently displaced. Visceroptosis is much more common in women than in men, and in women the breathing would appear to be less abdominal and more thoracic. There is 
little doubt that this is associated with the use of corsets and abdominal bands. These, by their interference with free abdominal action, ultimately cause atrophy and weakness of the abdominal muscles from pure disuse. A similar condition is seen in the adducted big toe with bunion formation due to atrophy of the abductor hallucis from the pressure of tight, badly fitting boots. Any hospital physician must have remarked on the difference between the male and female adult abdominal wall, which is so frequently in the latter flabby and toneless.

Hernia.-The pressure to which the abdominal viscera are subjected, both by the action of the diaphragm as well as by the abdominal muscles, together with certain alterations in the inguinal region associated with the erect posture, are regarded by many as the principal agents in the causation of femoral and inguinal hernia. These formations would appear to be almost peculiar to man amongst the mammalia. During the seventh month of foetal life the testes traverse the abdominal wall in an oblique direction, which led Cowper to observe that " the bowels were prevented from falling down by the obliquity of the spermatic passage." After birth the testes reach the fundus of the scrotum. Under ordinary circumstances the processus vaginalis or peritoneal prolongation associated with testicular descent should close after birth, above at the internal abdominal ring, and below close to the testes.

According to Hamilton Russell, inguinal hernia in the young is due to failure in the upper occlusion; the hernia is not due primarily, therefore, to straining or pressure, but to the fact that there is a congenital sac into which gut happens to have entered. " No sac, no hernia," is Russell's dictum. In the young, therefore, all that is necessary for cure of the hernia is to remove the sac without strengthening the inguinal wall, since in the absence of a sac the conjoined tendon is able to act as a powerful compressing agent on the inguinal canal. This simple principle has made the operative treatment of hernia in children almost a minor procedure. After nearly twenty years, during Iร 
which time recurrences could be watched, it would appear to have justified itself. Still two questions remain : why did the processus fail to become occluded, and why did intestine enter it? It cannot be denied that acquired herniæ do occur in adults accustomed to heavy weight lifting, though in such cases Russell would say that there was a preformed sac. In these, if the hernia were of old standing and the walls weakened by mechanical action, he would not hesitate to strengthen the canal by operation.

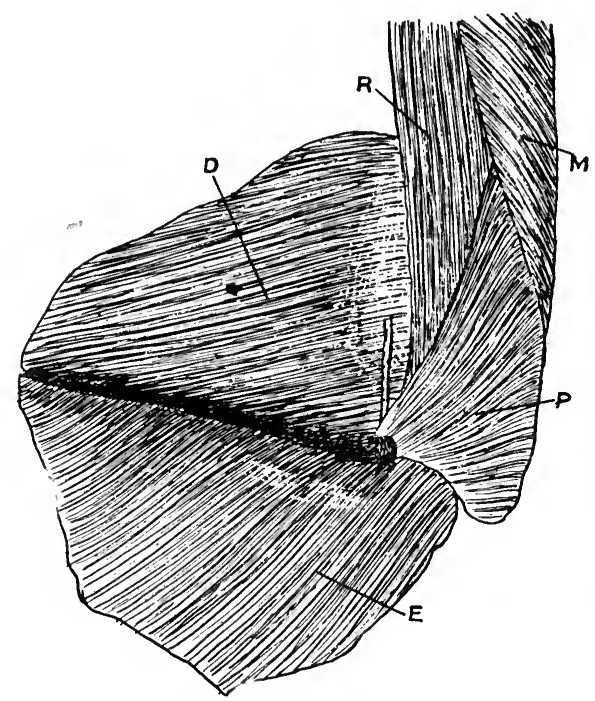

FIG. 96.-Inguinal region (male platypus).

M, Pectoralis major. P, Pyramidalis. R, Rectus abdominis. E, External oblique. Internal oblique and transversalis with attenuated portion corresponding to conjoined tendon. The muscular external oblique, which is thrown down, covers the attenuated area and is also prolonged over parts of the pyramidalis and rectus, so providing a muscular barrier to the formation of hernia. The testes are undescended. There is no splitting of the external oblique indicative of an external ring.

. On these questions some interesting light is thrown by a study of the abdominal wall of the Australian marsupials. In the monotremes (platypus and echidna) the testes are still intra-abdominal, and the marsupial bone is present in the lower part of the ventral abdominal wall, almost as if nature were anticipating testicular descent and seeking 
to improve the muscular action of this part. In the platypus the external oblique is muscular over an attenuated portion corresponding to the conjoined tendon, and is also prolonged inwards as a fleshy muscle over a portion of the superficial (pyramidalis) and deep recti. In the

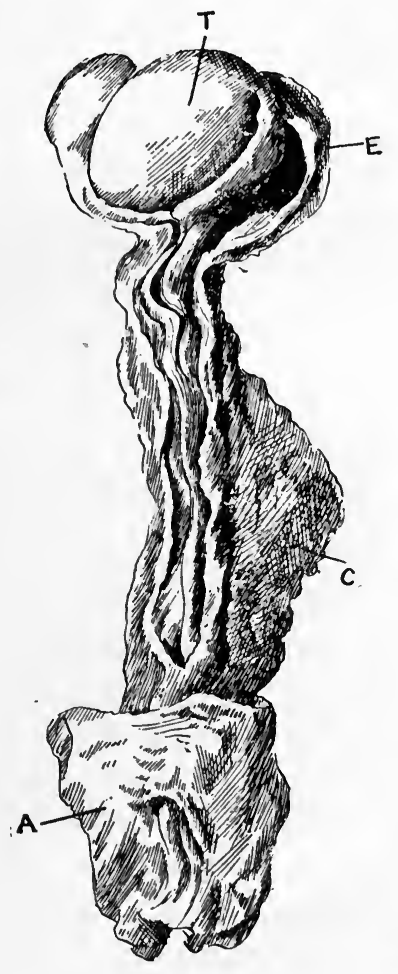

\section{V.C}

FIG. 97.-Dissection to show patent internal abdominal ring and canal continuous with the tunica vaginalis (male wombat).

A, Portion of peritoneal aspect of abdominal wall showing internal ring and cord entering it. c, Cremasteric muscle. T, Testicle. E, Open testicular sac.

marsupials also, the marsupial bone is present, and there is a well-defined pyramidalis (superior rectus) as well as the deep rectus. By means of the marsupial bone strength is given to the action of the abdominal muscles, and it forms a pulley round which the cremaster runs, passing in the 
male to the tunica vaginalis-testis and in the female to the mammary glands at the back of the pouch. Muscle has brought these extraordinary bones into existence. They are not primarily for the support of the prepenial scrotum in the male, nor of the mammary glands and pouch in the female, as is so often supposed. In the marsupials, however, the internal abdominal ring is patent; there is a canal of communication between the abdominal cavity and the tunica vaginalis. This is not universal however, and herein lies the interest. The internal rings in all female marsupials I have examined were found occluded. It is open in the male koala, kangaroo, wallaby, and wombat. Yet this patency is met with naturally in other members of the mammalia, as for example in rodentia, in the males of which order the testes undergo a periodical increase in size during the rut, and can pass from the abdomen into the scrotum and be retracted again into the belly. In the Tasmanian devil (carnivorous marsupial), there is occlusion of the internal ring in the male as well as in the female. In this animal it has been necessary to close the canal of communication, yet in a saltatorial marsupial like the kangaroo (springing often 25-30 feet), with a lax gastrointestine, nature has regarded the closure of the internal ring as of no importance. Compared with the abovementioned marsupials the Tasmanian devil is a feeble muscular type, and while the wombat thrives unprotected in the struggle against man, the former is now almost extinct. In this poor type, however, nature evolved external genitalia which are decidedly in advance of the monotremetous type of the other marsupials, and approach those seen in the higher mammalia. Comparing the Tasmanian devil in this respect and the wombat, the striking feature is the difference in development of the cremaster muscle. In the wombat, with a patent processus, the cremaster is flat, frequently over I inch wide, and would appear to act as a powerful compressing force on the attenuated conjoined tendon in its passage round the marsupial bone to the testes. In the Tasmanian devil the 


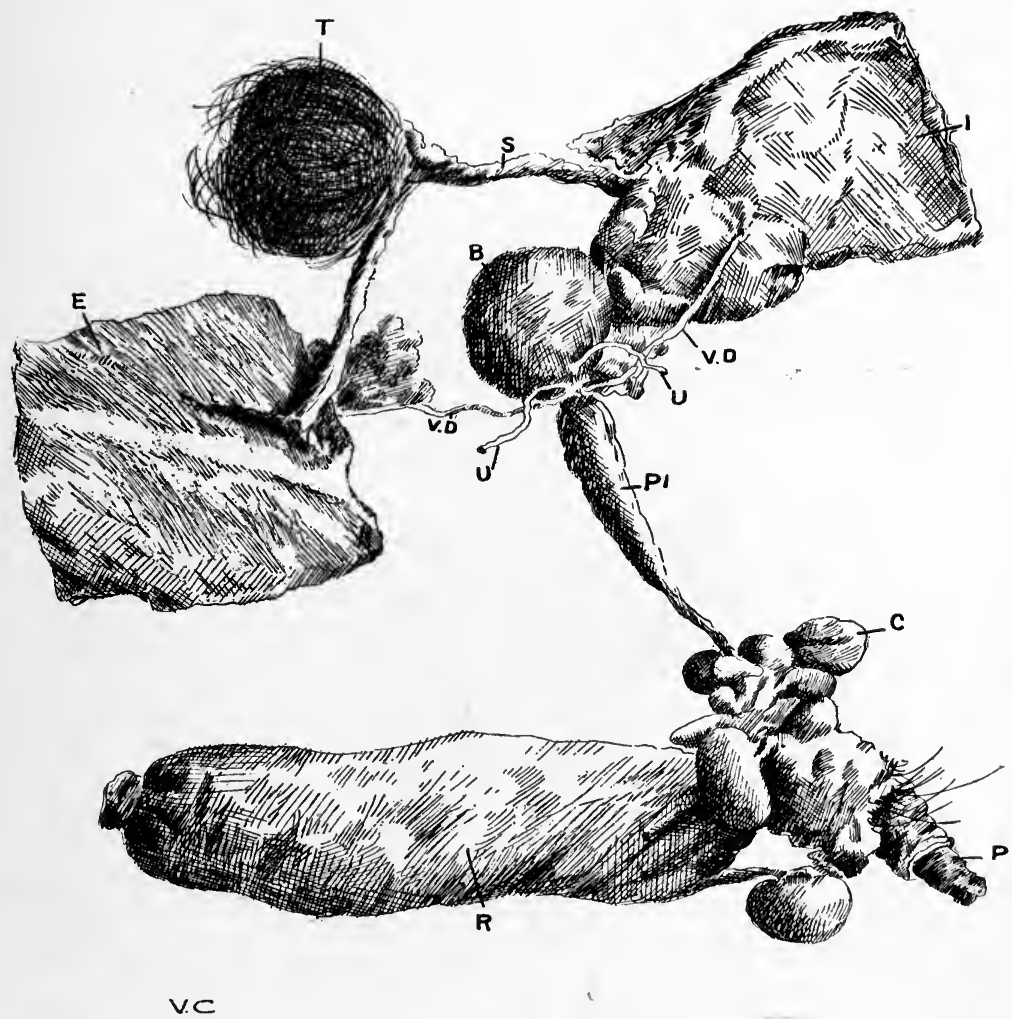

FIG. 98.-Dissection to show inguinal region (male Tasmanian devil)

(Dasyurus sarcophilus).

T, Testes. S, Spermatic cord. B, Bladder. V.D, Vas deferens. U, Ureter. P1, Prostate. R, Rectum. P, Penis. C, Cowper's glands. E, Inguinal region of abdominal wall (outer surface) showing epi-pubic bone. I, Peritoneal aspect of inguinal region of abdominal wall. 
marsupial bone and muscular arrangements are similar to those of wombat, but with the occlusion of the internal ring we have a rounded cremaster with poor relative development. Thus, in the marsupials some vital questions are raised concerning the causation of hernia which are calling for further investigation and research.

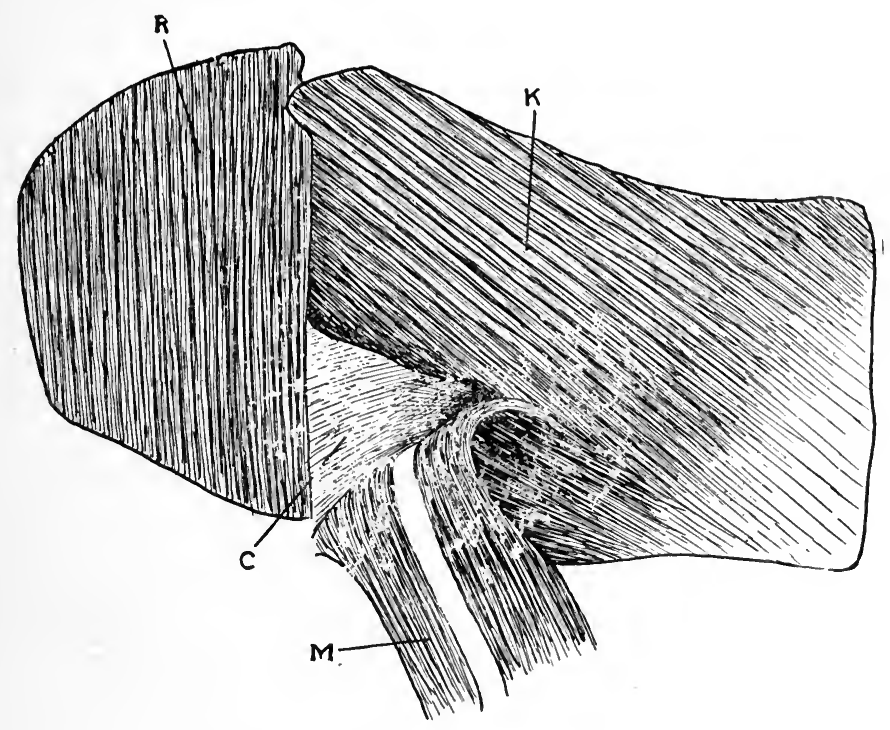

FIG. 99.-Inguinal region (male wombat).

The external oblique, pyramidalis, and epi-pubic bone have been reflected. $\mathbf{R}$, Rectus abdominis. K, Internal oblique. c, Attenuated area, formed by conjoined tendon, transversalis fascia, and peritoneum. M, Cord with cremaster muscle which is seen to be coming from internal oblique and transversalis muscles.

If the diaphragm - by its traction on the root of the lungs and its impaction against the abdominal viscera forcing them down and so enlarging the respiratory capacity of the chest-is the important factor in inspiration ; and if the abdominal muscles-by their contraction forcing the abdominal viscera upwards and backwards against the relaxed diaphragm and so causing its ascent with diminution of the thoracic capacity - are the important factors in expiration ; the thoracic muscles, which are really part of the two groups, also play some part in the respiratory mechanism, but much less than was originally supposed. 


\section{Function of the Thoracic Muscles}

The origin of ribs in the muscular sheet of the body wall must have been to improve muscular action, since these allowed the development of multiplicity of muscles, and hence diversity of function. Throughout the ancestral world bone is dominated everywhere by muscle. The instances of its subservience are numerous and scattered. Thus, muscle dominance produces a broadening of the bone surface where muscle attachment is required, and a narrowing or rotundity where such attachment is diminished; compactness or lightness of bone where strength or otherwise is requisite, and bony ridges and projections to afford leverage.

In the case of the thorax, however, not only do the ribs give attachment to muscle and protect organs which are in a constant state of motion from the cradle to the grave, the heart and lungs, but they accommodate themselves to the motion of these organs, and also to motions of the body, such as forward or backward movements or movements laterally. All this would be impossible if we had plates of bone instead of a number of movable parts, or if the ribs were completely formed of bone from the spine to the sternum. Fortunately, elasticity and adaptability are conferred on the ribs by the interposition of elastic and bending cartilage anteriorally between the ribs and the sternum. In this way the chest and its contents are rendered almost proof against blows and jolts to which the body is liable, and one has only to recall the liability to fracture of the ribs in the aged in whom ossification of cartilage occurs. We are apt to imagine that the thoracic wall has been called into being for the protection of the heart and lungs. This is not so. The testes are not protected by a bony or cartilaginous covering, nor have the abdominal muscles ribs in their interstices for the protection of the gastro-intestine. The great point to be borne in mind as to their necessity is the base they afford for muscle attachment-muscles from the loins, muscles to the neck-abdo- 
minal muscles, and large muscles like the pectorales, latissimus, and trapezius for the movements of the upper limbs. When we find, as in the aged, life proceeding with little restraint in spite of complete ankylosis of the ribs interfering with their motion ; it raises the question whether the intercostal muscles will ultimately be replaced by fibrous bands between the ribs, although in the present state of evolution muscles are a necessity. The great thoracic enlargement on inspiration which athletes are prone to demonstrate is not, after all, of such vital importance as is generally regarded. It is worth remembering that the actual enlargement of the chest in the antero-posterior and transverse diameters in quiet respirations does not exceed $5 \mathrm{~cm}$. The centre of motion for the action of the ribs is at their articulation with the spine. There are two movements-one, elevation or raising and depression or falling anteriorally, so as to allow increase or diminution in the antero-posterior diameter (the vertical being increased by diaphragmatic action). The other is the motion of the rib on its own axis, which is a line drawn between the two extremities to increase or diminish the transverse diameter, since by this motion elevation of the middle portion of the rib takes place. It is owing to the elasticity of the chest wall that these motions essential to respiration, both inspiratory and expiratory, can be carried out. Though, as stated, the diaphragm and the abdominal muscles are the prime muscles in respiration, the thorax, through its muscles, also moves in unison with these. The contraction of the diaphragm in inspiration allowing of increase in the vertical diameter of the thorax, is associated with relaxation of the abdominal muscles-the tractors of the thorax. This relaxation also permits of the action of the intercostal muscles, which elevate the ribs and sternum, and thus the thorax is enlarged in all its diameters. In this they are assisted by the levatores costarum, infracostales, and serratus posticus superiorall inspiratory muscles. With expiration, the intercostal muscles are relaxed as well as the diaphragm. Although possibly the elastic recoil of the lungs and the weight and 
elasticity of the chest wall (since the ribs are always endeavouring to maintain the equilibrium between inspiration and expiration) are factors, the essential one in my opinion is the action of the abdominal muscles. They act not only on the viscera, but also, owing to the traction they exert, on the lower half of the thorax; in this they are assisted by the triangularis sterni. This muscle is a continuation up of the transversalis abdominis, and its action is to depress the ribs, draw down the costal cartilages, and so to act as a muscle of expiration. It is questionable whether the serratus posticus inferior has any respiratory function. Probably Riolan was right, centuries ago, when he regarded this muscle as a binding and strengthening force to the muscles of the back, similar to the annular ligaments at the ankle. There is little doubt, as Haller taught, that the internal intercostals are not expiratory muscles, but that both sets are inspiratory. The division of the intercostals into two sets is really artificial, for it is a mechanical arrangement by which nature obtains velocity of action at the expense of power. As in the case of the abdomen, a single straight sheet of muscles between the ribs would be insufficient. By the obliquity of the intercostals we have traction through the maximum space with the minimum contraction. Through this arrangement less muscular effort is required to approximate the ribs. The action of the intercostal muscles, which we must remember are essentially muscles of ordinary inspiration, would appear to be as accessories in connection with forced inspiratory efforts as in running or violent bodily exercise ; efforts which are impossible in old age, when thoracic elasticity is greatly diminished or absent. In violent exercise intercostal action is necessary as an aid to the action of the diaphragmatic pump, so as to permit of the rapid aeration of the blood essential to the effort.

Phrenic Paralysis.-Only once have I seen a case of paralysis of the diaphragm (post-diphtheritic), and it was interesting to note that the thoracic muscles failed as inspiratory agents to compensate adequately for the impair- 
ment of diaphragmatic action. Aeration was defective, there was little abdominal movement, the lungs were incompletely emptied, congestion of the bases followed, with subsequent consolidation and gangrene. In these rare cases it is presumably a " paresis," not a " paralysis," of the diaphragm that is present. With paralysis of the diaphragm it is inconceivable that life can be prolonged. If both phrenic nerves (the main motor nerves of the diaphragm) be divided, death results-the other inspiratory muscles being unable to maintain respiration effectively.

Epigastric Retraction.-Associated with the dyspnœa of a severe case of laryngeal diphtheria is the so-called " epigastric retraction," which is an indication for immediate intubation by O'Dwyer's tubes or for tracheotomy. The inspiratory muscles, namely the diaphragm and intercostals, are working ineffectively. Owing to the diminished entrance of air into the lungs through the mechanical obstruction of the larynx, the expansion of the thorax and lungs is at a minimum. There is little downward and forward movement of the abdominal viscera. The lessened pressure within the thorax, owing to the diminishing entrance of air, is insufficient to counteract the pressure of the outside atmosphere, and supraclavicular, epigastric, and intercostal compression result. The pressure within and without the thorax is unevenly balanced.

If there were complete obstruction to the entrance of air into the lungs, no expansion of the thoracic cavity could occur, the muscles of inspiration being unable to counteract the atmospheric pressure on the outside of the chest. 
$4=-2 x$ 


\section{N D E X}

Abdomen, inflammatory conditions of, movement of thoracic at expense of abdominal muscles in, 240

Abdominal muscles, 228

comparative anatomy, $237,23^{8}$

development, 238

function, 237,247 modification in, 238

in platypus, 230

poliomyelitis affecting, rest treatment, 239

relaxation, associated with contraction of diaphragm, 247

relaxed and stretched, action of, in respiration, stimulus to, 234 support of viscera by, 239

Abdominal and thoracic muscles, action of, 234

respiratory movements effected by, 234

Abdominal organs, effect. of diaphragm on, 236

ribs in crocodile, $23 \mathrm{I}$

ring, internal occlusion in female marsupials, 244

potency in marsupials, 243,244 viscera, respiratory movements, 236

wall, muscles of, 228

Abduction, application in paralysis of quadriceps, 162

essential movement of, 46

or adduction frame, I 40

splint, upper limb, advantages of, 227

description, 59

in loss of muscular power of deltoid, and trapezius, 58, 59

in musculo-cutaneous paralysis, 80

in separation of epiphysis of humerus, 227

Abductor hallucis, origin and insertion, $\mathrm{r}_{5}$

weakened state in hallux valgus, I87
Abductor minimi digiti, action, origin and insertion, I9I

Abductor pollicis, 99

and adductor hallucis, actions contrasted, $\mathrm{r} 86$

Abductors of little finger, 97, 98

Acrobats, best illustration of action of flexors and extensors of ankle seen in, 176

muscular feats of, I $50,212,216$, 217,218

Acromion, action, 57

Adductor brevis, origin and course, 136, 138

contraction, result, 68

hallucis, oblique portion of, 185 transverse portion of, 185 , I 86

longus, origin and course, 136 and magnus, work of, 139

magnus, insertion, 138,139 origin, 136

of little finger, 98

Adductores pollicis, 98, 99

Adductors, action on thigh, 139 spasmodic contraction in spastic diseases in children, $14^{\circ}$ surgical treatment, $\mathrm{r}_{4} \mathrm{O}$

Adhesions, breaking down of, 27

African races, os calcis in, $\mathrm{I}_{7} \mathrm{I}$

Albinus, biventer cervicis, 203 distinction between splenius capitis and splenius colli, 202

Aluminium cock-up splint in graduation of flexion in musculospiral paralysis, I 2 I

Amphibia, abdominal situation of lungs in, 235

muscles of body wall in, 237

Anconeus, order and position of origin, I I I

Ankle-joint, diseased or injured, rest of, 224

extension backward of rigid body at, 175,176

flexion and extension at, principles of leverage in, 174 
Ankle-joint, flexion at, in talipes calcaneus, 176

flexors of, paralysis, 23 shortening of, 27

flexors and extensors, best illustration of action, I 76

maintenance of equilibrium at, in respect of flexion and extension, in deformities of foot, I92, I 93

position for rest or recovery, or for ankylosis, 224

Ankylosis of joints, 222, 223, 226, 227

prevention in upper extremity more important than in lower, 223

Annuentes, 203

Anthropoids, deltoid function in, I 7

dependence upon fore limb for support, I6r

femur in, 136

peroneus brevis in , I8I

Ape, orthograde posture of, 17

quadriceps in, $16 \mathrm{I}$

Appendicitis, movement of thoracic at expense of abdominal muscles in, $24^{\circ}$

Arc shoe attached to single Thomas's splint for rest of knee-joint, 225

in deformities of foot, 197, I98

Arc splint, use in rest of anklejoint, 224

Arcuate ligaments of diaphragm, 232

Arm, abduction of, in loss of muscular power of trapezius, $5^{8}$

ability to raise, in man, $5^{\mathrm{O}}, 5^{\mathrm{I}}$

elevation, to rest triceps, in musculo-spiral paralysis, I I 7

Athletes, bending back of rigid trunk and thigh at knee-joints by, I 50

extension backwards of rigid body at ankle-joint in, I 76

Atlas and occiput, articulation between, flexion and extension at, example of first order of leverage, 205

articulation with axis, 200 with occiput, $2 \mathrm{OI}$

Axis, direct connection with occiput, 201

Back and loins, muscles moving, 2 I 3

dorso-vertebral, or extending, 2 I 4
Back-splint and foot-piece in rest of foot, in paralysis following injury of internal popliteal nerve, I95

Balance, uneven, between elongation and contraction, 39

Bandage sling, use in loss of muscular power of serratus, 60

Bartholin, relations between diaphragm and transversalis, 232

Bell, Sir Charles, source of muscular action, I6I

Biceps brachii, action, 75

brachialis compared with, 7 I

in Malabar squirrel, 78,79

in wombat, 78,79

insertion, 75

long head of, division avoided during excision of shoulder joint, 75

involvement in diseases and injuries of shoulder-joint, 79

position of rest in musculocutaneous paralysis, 80

origin, 73

paralysed, old cases with con. tracted pronator teres, treatment, 83

recovery, how effected, 82,83

state of, in pronation and supination of forearm, 76

supination, 78

Biceps cruris, heads of origin, 148

in monotremes and marsupials, 152

insertion, 148

of platypus, I34, I35

outward rotator, I $5 \mathrm{I}$

portion in gorilla more distinct

than in man, I 53

Biventer cervicis, 203

Bland-Sutton, Sir J., Gimbernat's ligament and triangular iascia, 230

Body, human, falling forward, muscles preventing, 2 I 8

superincumbent pressure of, how supported, I 59

trunk of, point at which upper extremity connected with, 57

relation of limb action of upper extremity to rigidity of, 234

upright position, muscles neces sary for maintaining, I75

wall, muscular system of, in amphibians, 237

Bone, insertion of muscle into, 3

relation to muscle, 4 
Boot, shape of, in cases of hallux valgus, 187

Brachialis, antagonist of, $7 \mathrm{I}$ character in man, $7 \mathrm{I}$ compared with biceps, 7 I function, $71,72,75,76$ how rested in musculo-cutaneous paralysis, 80

" moment of force" of, 8o, 8r, 82 origin the insertion, and insertion origin, 72

paralysis of, test for, 9 reverse action of, 72 stimulus to contraction of, $4 \mathrm{I}$

Brachio-radialis, action, 73 in marsupials, 73 origin and insertion, 73 , III "survival value" of, 34

Bundles. See Fasciculi

Bunion of big toe, 186

Calcaneus. See Os calcis

Caliper splint, Thomas's, for knee joint after commencement of ambulation, 226

Cardboard, powdered, use of, for rest of paralysed limbs, 60,65 , $80,120,123$

Cervical region, flexion and extension, movements free in, 206 rib, 31, 32

Cetacea, diaphragm in, 232

Children, inguinal hernia in, $24 \mathrm{I}$ operative treatment, 24 I spastic diseases in, impediment to walking in, $\mathrm{I}_{4} \mathrm{O}$ paraplegia of, contraction of gracilis in, ${ }^{4} 8$

Cock-up splint, for rest of wrist joint, II 8, II 9,226

Complexus, action, 202, 203 origin and insertion, 202

Connective tissue, 2

Conoid, 57

Contracted muscle, 22

Contraction, chronic, 22, 24 deformities produced by, 25 treatment of, 26

mechanism of, 46

physiological action of, 36

stimulus to, 40

uneven balance between elongation and, 39

Contractions, breaking down of, 27

Coraco-brachialis, 55

Coraco-clavicular ligaments, 57

Cornual cell, rest of, I9

Costalis cervicis, 206, 207

Cowper, abnormality in rectus abdominis, $24^{\circ}$
Cowper, comparative weight of extenders and benders of knee, I 59

discoverer of inter-spinales, 209

Cremaster muscle in Tasmanian devil and wombat, 244

Crocodile, abdominal ribs in, $23 \mathrm{I}$

Crureus (vastus intermedius), I 55

connection of vastus internus or medialis with, I $54, I_{55}$

Death, elasticity not affected by, 38

Deformities produced by chronic contractions, 25

Deltoid, 52, 53, 54, 57

abduction, movement in arc of, $61,62,64,65$

action of, 14

characteristics of, $5 \mathrm{I}, 52$

development in man, $5^{\mathrm{I}}$

function, 45, 57

in anthropoids and marsupials, $\mathrm{r} 7$

insertion, 54

loss of muscular power in, position of rest for, 59

origin, 52

paralysed, point of support of, 2 I re-education, $62,63,64$

paralysis of, 23

test for, $3^{\circ}$

Diaphragm, arcuate ligaments of, 232

comparative anatomy, 232, 235

contraction associated with relaxation of abdominal muscles, 247

crura of, 232

dimensions, 23 I

direct antagonists of, 237

effect on abdominal organs, 236

function, 235,247

inequality of action between right and left sides, 237

movement of, avoided in abdominal inflammatory conditions, $24^{\circ}$

muscles of, $23 \mathrm{I}$

paralysis, rarity, 248, 249

periphery, $23 \mathrm{I}$

principal muscle of inspiration and respiration, 234

upper and lower surfaces, $23 \mathrm{I}$

“Dropped toe" deformity, I83

" Dropped wrist," definition of, I I9

metal splint, Thomas's, I I 8

prognosis, test for, II9

trẹatment, I I 9 
Elastic body, action compared with muscular action, 38

movement by shortening, 38

Elastic power, I

Elbow joint, diseased or injured, rest of, angle of flexion for, 227

best position for, 226, 227

wrong methods for, 226

extension, how best effected, 77

to rest triceps, II 7

flexion of, differentiated - from supination of forearm in marsupials, 79

in ulnar paralysis, ror

how best effected, 77

how permitted, 77

in musculo-cutaneous paralysis, 80

not position of physiological rest, 80

flexor of, 75,76

forces acting on, $4 \mathrm{I}$

muscle crossing, 75

Elongation, muscular, as mechanical action, 37

power of, 38

uneven balance between contraction and, 39

Epigastric reaction, 249

treatment, 249

Epi-pubic or marsupial bone, 230

Equilibrium muscular production, 39

Erect posture, 4, I6

in man, characteristics of, $49,5^{\circ}$

Erector spinæ, action of, 216

Erectores spinæ, action, extreme instance in acrobats, 216,217

antagonists of, 218

contraction, 218

position and attachment, 2 I4

European races, os calcis in, I $7 \mathrm{I}$

Evolution in relation to function of quadriceps, I60, I6I

muscle function in, 17

of muscular action, I4

Extensor brevis digitorum, fulcrum, 183

insertion of tendons, 182,183

muscular bellies of, $\mathrm{I} 88$

origin and course, 182,188

tendons of, 188

Extensor brevis pollicis, II2

Extensor carpi radialis brevis, III longus, III

carpi ulnaris, III, II 2

communis digitorum, III, II 2

action of, II4, II 5

metacarpo-phalangeal joints, II 6
Extensor communis digitorum, origin, II4

tendons of, II 4

testing for recovery of, in musculo-spiral paralysis, I2 I

indicis, II2

longus and brevis digitorum, special function of, I 89

longus digitorum, I $84, \mathrm{I} 88$ tendons of, 188

longus hallucis, action, 183

fulcrum, 183

held down by ligaments, 184

insertion of tendon, I82

origin, 182

longus pollicis, IOO, II 2

insertion of, II2

minimi digiti, I I I, I I 2

ossis metacarpi pollicis, II2

rest in musculo-spiral paralysis, II 7

tendon, movement over condyles of femur, 155

Extensors, action of, 39

of fingers, order and position of origin, III, II 2

re-education in, 107, 108

of foot, 170

of leg, I 54

stimulus to contraction of, $4^{\circ}$

External oblique (descendents), aponeurosis of, 228, 229

fleshy muscle of, 228

muscle fibres of, 228

situation, 228

Extremity, upper, adequate fixation of one joint, how best secured, 227

limb action in relation to rigidity of trunk, 234

paralysed, re-education in infantile paralysis, 65,66

prevention of ankylosis in, more important than in lower, 223

Fasciculi, muscular, 2

Femur, body weight when standing shared by each, I 30

condyles of, movement of extensor tendon over, I 55

in anthropoids, 136

middle, body-weight transmitted through, when standing on one leg, 130

movements of, 127

muscles performing, I 27

where performed, I27

neck of, dimensions, why necessary, $z^{\circ}$ 
Femur, rotatory movements of, when impossible, I 44

Fibre, elastic, elongation of, $3^{8}$

Fibres, cylindrical, I

direction of, 13 elongated, tightening of, 27

tension of, 47

Fibro-cartilage, formed in tendon of peroneus longus, I73

Fibrosis, luetic, 28

Fibula, adaptive changes in, 3I, 32 compared with tibia, I7

congenital defects of, treatment, I 78, I 79

non-existence as separate bone in kangaroo, ${ }_{17} 8$

Finger, dorsum of, arrangement of motor forces on, II 5

little, small muscles of, nerve supply, 97

middle, double supply of profundus tendon to, IO8

Fingers, abduction and adduction of, 99, IOO, IOI

condition of, in partial ulnar paralysis, 109

extension in musculo-spiral paralysis, II 7

extensors of, order and position of origin, I I I, I I 2

re-education, I07, 108

flexion favoured by position of extension of wrist, in ulnar paralysis, ro9

flexion of, recovery in median nerve paralysis, re-education for, 95

flexor leverage, improvement by extending wrist, 96

flexors of, $85,87,90$

lateral action, imaginary line for, rgr

middle and index, double nerve

. supply, ro7

movements in median, ulnar, and musculo-spiral paralysis, I 24-6

muscles of, re-education in ulnar paralysis, $\mathrm{IO}_{4}$

rest of, in re-education of interossei, ro8

weakened, in musculo-spiral paralysis, extension, how aided, I 2 I

Flat foot, 187

Fleshy muscles, stability and strength of, 3

nature of muscle, 3

Flexor accessorius, function, 189

origin and insertion, 189
Flexor brevis digitorum (perforatus), action, I9O, I9I

tendons of, I9O

Flexor brevis hallucis, tendons of, 184

Flexor brevis minimi digiti, function, 190

origin and insertion, I9o

Flexor brevis pollicis, 98

Flexor carpi ulnaris, Ior

nerve supply, 97

origin, 97

communis, antagonists, 89

longus digitorum (perforans), action, I9O, I9I

insertion of tendons, 189

origin and insertion, 189

Flexor longus hallucis, use of, $\mathrm{I} 85$ origin, 184

Flexor profundus, tendons, 87,88

and flexor sublimis, reciprocal leverage by, to improve flexion power, 88

digitorum, origin, 97

nerve-supply, 97

Flexor sublimis, tenđons, 87,88

Flexores capitis, $2 \mathrm{O}_{5}$

Flexors, action of, 39

contracture of, in musculo-spiral paralysis, I I 8, I 20,124

division of lumbricales, 124-6

examples of pulleys, 9

of ankle, paralysis of, 23

shortening of, 27

of fingers, $85,87,90$

of foot, I 68

of knee, chronic shortening of, 23

of leg, ${ }_{4} 6$

antagonist, 158

of wrist, testing of, 29

reverse action of, 5

Food, stimulus to intestinal contraction, 38

Foot and hand, specialization of, function in, compared, I92

and toes, muscles acting on, rest and re-education in sciatic nerve paralysis, I92, 194, 195

deformities, acquired, I79

following poliomyelitis, I 76

maintenance of equilibrium at ankle as regards flexion and extension in, 192, I93

surgical treatment, I97

useful form of splint for, I97, I98

dorsal and plantar surfaces of, impairment of function, how produced, 193

eversion, 179 
Foot eversion in kangaroo, how prevented $\mathrm{I}_{7} 8$

with extension, muscle producing, 177

with flexion, muscles producing, I77

extension, muscles effecting, 170 , I 73

flexion, muscles effecting, I68, I 73

front of, action, how strengthened, I 86

human, eversion and inversion of, I7

interossei in, I9I

inversion, $\mathbf{I} 77$

with extension, muscle producing, I 77,194

with flexion, muscles producing, I 77

inverters and everters, action of, 197

inversion and eversion in flexed and extended position, in connection with lateral movements, 197

in koala, $\mathrm{I}_{7} 8$

maintenance at right angle, in prevention of deformities, I97

muscles acting on, I68-8I

of kangaroo, 32

of koala, 33

rest treatment in case of shell wound of upper leg involving peroneal nerve, 193, 194

in paralysis following injury of internal popliteal nerve, I 95

right, "paralysis" following infantile paralysis, recovery from after rest and re-education of muscles, I95, 196

splint, special form useful in treatment of deformities, I97, I 98

" springiness" on, importance of, I 85

Forearm, branches of ulnar nerve arising in, 97

flexors of, test for action, 29

ischæmic " paralysis" of, avoidance, 227

paralysis of pronators, 23

position of, in testing for deltoid recovery or in commencing reeducation, 63

position of over-supination in musculo-cutaneous paralysis 80
Forearm, position to secure rest in musculo-spiral paralysis, I I 7 pronation and supination of, state of biceps in, 76

in flexed position, how effected, 77

supination of, differentiated from flexion of elbow in marsu pials, 79

how effected, 77

Fore-limb, ability to raise in marsupials, $5^{\mathrm{I}}$

as means of support, I7

dependence of anthropoids upon, for support, I6r

Fracture of patella, production by quadriceps, I 59

Fulcrum, definition, 39

Function (muscle), evolutionary law of, $\mathrm{I} 7$

(muscle), re-education of, 20, 22 specialization of, 44

Gastrocnemius, course, I7 I

function of, I73, I74, I75

origin and insertion, I 70

reverse action of, 42

tendons of, $\mathrm{r}_{7} \mathrm{O}$

Gemellus, superior and inferior origin, $\mathrm{I}_{42}$

insertion, 142

Gimbernat's ligament, 230

Gluteus maximus, action in rising from sitting to erect posture, I 34

functions of, I 34

of platypus, I34, I35

origin and tendinous insertion, Iy I

relaxation and elongation, 129 , I 49

relaxation with contraction of ilio-psoas, effect, $I_{5} 8$

size in man, I3I, I32

Gluteus medius, action of, I 4 I

muscle assisting, I $4 \mathrm{I}$

Gluteus minimus, action of, I44 origin and insertion, I34, I44

Gorilla, erect posture, I 36

portion of biceps cruris in, $\mathrm{I}_{53}$

Gracilis, contraction in spastic paraplegia of children, I $^{8} 8$

development in platypus, $\mathbf{I}_{5} \mathbf{I}$

fulcrum of, 147

origin, insertion, and function, $\mathrm{I}_{4} 6, \mathrm{I}_{47}, \mathrm{I}_{4} 8, \mathrm{I}_{5} \mathrm{I}$

Grafting in paralysis, 18

Gunshot wound of great sciatic or peroneal nerves, cause of "paralysis" of leg and foot muscles, I 93 
Hall, Marshall, reflex movement from irritation, $16 \mathrm{I}$

Hallux, muscles on, 182

Hallux valgus, 187

operation in severe cases, 187

reason for greater frequency in women, 187

shape of boot to be worn in cases of, 187

treatment, 187

weakness of abductor hallucis in, 187

Hamstrings, true cause of tight feeling of, I 49

Hand, branches of ulnar nerve arising in, 97

radial or ulnar deviation; reeducation for, $\mathrm{Ir}_{4}$

rest of, in paralysis of wrist extenders, i 18

and foot, specialization of function in, compared, 192

Hand-piece, application in ulnar paralysis, $\mathrm{IO}_{3}$

Hand splint, Thomas's, for rest of wrist joint, 226

Head, lateral movement, 2 I 2

movements on spine, 201

muscles moving, 200

Hernia, femoral, cause, 24 I

in children, operative treatment easy, $24 \mathrm{I}$

inguinal, cause, $24 \mathrm{I}$ in children, cause, $24 \mathrm{I}$

ventral, development, 239

Hip-joint, adduction and abduction at, 136

alteration of angle at, $\mathrm{I}_{5} \mathrm{I}$

disease, psoas contraction as diagnostic of, 132

flexion at, 130

during sitting, I 50

how effected, $13^{\circ}, 1_{5}{ }^{8}$

and extension of, effects on, spine, ${ }^{1} 32$

forcible reduction of, 133

rotatory movements at, limited, I 44

subject of more continuous movement than other joints, 129

and knee joint, flexions of, 146 independent movements, 146 , 147

Horizontal bar, elevation of body on, example of reverse action of brachialis, 72

Humerus, check on movement upward, 57

muscles moving, 52

insertions of, 53
Humerus, rotation of, $5^{8}$

separation of epiphysis of, treat. ment, 227

Iliacus, origin and insertion, 130

Ilio-costalis, course and insertion, $2 \mathrm{I} 4$

Ilio-psoas, I 3 I

action of, in movement of leg, in recovery of paralysed quadriceps, 165,166

when body bent forward at hip joint, 149

contraction, with relaxation of gluteus maximus, effect, ${ }_{5} 8$

Index and middle fingers, interossei of, double nerve supply, $\mathrm{IO}_{7}$

Inferior oblique, action, 204

course, $2 \mathrm{O}_{4}$

Inflammation, rest the basic treatment of, r9, 2 I

Inflammatory conditions of abdomen, movement of thoracic muscles in, $24^{\circ}$

Infracostales, 233

Infraspinatus, origin and insertion, 54

Inguinal region in male platypus, 242

in male wombat, 245

Injury, muscle rest after, 20

Insertion of muscle, 2, 5

adaptation to alteration of distance, 43

Inspiration, principal muscle of, 234

Intercostal muscles, action, 247, 248 fixed point for action of, 2 I 3

Intercostals, external, 232, 233 internal, 233

Internal oblique (ascendens), aponeurosis of, 229

insertion, 229

situation, 229

Interossei, action, 90 tests bearing on, roo

long tendons of, roI

Interossei in foot, dorsal, I9I action, 192

plantar, I9I action, 192

Interossei in hand and foot, importance compared, I9I

Interossei of index and middle fingers, double nerve supply, 107

Interossei, palmar, function of, 99 and dorsal, 98

functions of, 98,99

recovery of, in ulnar paralysis, 106

testing for, I $2 \mathrm{I}$ 
Interossei, re-education, point to be observed in, ro8

on splints in ulnar paralysis, method, I ro

rest of, in musculo-spiral paralysis, 117

neglect in ulnar paralysis, results, r ro

weakness in ulnar paralysis, $\mathrm{rO}_{4}$

Interosseous muscle contraction, result of, IOI

Interphalangeal joint, first or proximal, flexion at, effect $\mathrm{cn}$ distal phalanx, IOo

Interphalangeal joints, extension at, roo

in partial ulnar paralysis, rog

inability to extend at, in ulnar paralysis, $\mathrm{IO}_{4}, \mathrm{IO} 5$

recovery of power, I05, 106

Inter-spinales, $2 \mathrm{I}_{5}$

action, 209, 217

discoverer of, 209

Inter-transversales, 2 I 5

action, 209, $2 \mathrm{I} 7$

area of extension, 208

region where best developed, 208 , 2 I 7

Intubation in epigastric reaction, 249

" Irreparable damage," I5

Ischæmic paralysis, 28

of forearm, avoidance, 227

Joint, muscular opponents of, 40

Joints, ankylosis of, 222, 223, 226, 227

diseased, treatment by counteraction of active and passive forces, 222, 223

treatment by perfect rest, 222, 223

results aimed at in, 223

dominated by muscles acting across them, 222

fixation of, anatomical considerations in, 222-7

functions, 222

relation of ligaments to, 7

relation to muscle, 4

several, muscles passing over, 43

Jumping, muscles employed in, I $74, \mathrm{r} 80$

Kangaroo, ability to raise forelimb in, 5 I

absence of tibialis posticus in, ${ }^{7} 78$ brachio-radialis in, 73

buttock, thigh, and outer leg,

dissection showing, 137

erect posture in, $5 \mathrm{I}$, I $3 \mathrm{I}$, $\mathrm{I}_{32}$
Kangaroo, foot and leg of, 32 function of sartorius in, 152 great size of psoas minor in, r 3 I

length of tendo-achillis in, 24

muscular system of, 4

non-existence of fibula as separate bone, in 178

peroneus longus almost nonexistent in, I8I

saltatorial, eversion of foot in, how prevented, I $_{7} 8$

tibialis anticus in, 178

tibia and fibula in, compared, I7

Keith, A., F.R.S., comparative anatomy of diaphragm, 235

Innee joint, chronic disease of, with backward displacement, outward rotation of joint in, $I_{5} I$ crossed by gastrocnemius, I7 I diseased or injured, position for rest or ankylosis, 225

rest of, splint for, 225

splint for, after commencement of ambulation, 226

dissection of inner side in wombat, I 52

extenders and flexors of, weight compared, I 59

flexion at, how constituted, $5_{50}$ of rigid body at, 216,217

and extension in re-education of paralysed quadriceps, $\mathrm{I}_{5}$, I66, I67

flexors of, action, I 5 I how evidenced, ${ }^{5} 5^{O}$

chronic shortening, 23

not crossed by soleus, I $7 \mathrm{I}$

protection of, I 55

rotation inward at, $I_{5} \mathrm{I}$

movement at, $\mathrm{r}_{5} \mathrm{I}$

outwards at, $\mathrm{I}_{5} \mathrm{I}$

stiffening of, to maintain erect posture, how effected, $5^{\circ}$

and hip joint, flexions of, 146 independent movements, 146 , 147

Knee joints, bending back of rigid trunk and thigh at, by acrobats and athletes, I $5^{\circ}$

Koala, ability to raise fore-limb in, $5^{\mathrm{I}}$

brachio-radialis in, 73

deltoid function in, 17

foot and leg of, 33

inversion and eversion of foot in, 178

peroneus brevis in, I 8 I

strength of semi-membranosus and semi-tendinosus in, ${ }^{I_{5}} \mathrm{I}$ 
Lateral rolling, how produced, 2 I 9

Latissimus dorsi, origin and insertion, 54,55

Leg and foot, muscles of, causes of "paralysis" in, 193

Leg, extensors of, I $45,{ }_{54}$ flexion and extension on thigh when sitting, $15^{\circ}$

flexors of, 145.146

movement of, action of iliopsoas muscle in, in recovery of paralysed quadriceps, I65, I66 muscles acting on, $145^{-6} 7$

of kangaroo, 32

of koala, 33

upper, shell wound of, involving peroneal nerve, incapacity resulting from, I93

Levatores costarum or supracostales, origin and insertion, 233

Leverage, first order of, 205

Levers, examples of, 12

Ligaments, elastic properties of, 38 lateral, patellar, I55

of extensor longus hallucis and extensor longus digitorum, 184 relation to joints, 7 shortening of, 24

Limb, right lower, supporting trunk in erect position, 128

upper elevation, mechanism, $57,5^{8}$ inutility in infantile paralysis, cause, 9 I

Limbs, lower, of man, how differing from those of other mammals, I30

paralysed, require gentle handling, 60

Linea alba, 228, 229, 230, 23I

Lineæ semi-lunares, $23 \mathrm{I}$

transversæ, $23 \mathrm{I}$

Lister's splint. See Splint, Lister's

Loins. See Back and loins.

Longissimus dorsi, attachment and -insertions, 2 I4, 2 I 5

Longus colli, action, 209, 2 I I, 2 I2 area of extension, 209

three divisions of, 209

Luetic fibrosis, 28

Lumbar region, flexion and extension free in, 206, 213

flexion or extension at, results, 219

inter-transversales well developed in, $2 \mathrm{I} 7$

lateral movement at, 2 I3, 2 I9

rotation absent in, 213

semi-spinales not found in, $2 \mathrm{I} 7$

Lumbricales, origin, 190

digital, action, 89,90
Lumbricales, digital, not extensors of middle and distal phalanges, 90 origin and insertion, 89

division, in musculo-spiral paralysis with contracture of flexors, 124

inner, 98

relaxation and elongation in musculo-spiral paralysis, II 7

Lungs, abdominal situation in amphibia, 235

Malabar squirrel, biceps brachii in, 78,79

Man, ability to raise arm in, 5O, $5^{\mathrm{I}}$ development of deltoid in, $5 \mathrm{I}$ erect posture of, basic factor in maintenance of, I59, I6o characteristics of, $49,5^{\circ}$ principal muscular changes in connection with, I3I

latest muscular functions to be acquired by, 50

shoulder region, in, muscles, 52

Marsupial or epi-pubic bone, $23^{\mathrm{O}}$, $242,243,244$

Marsupials, ability to raise forelimb in, $5 \mathrm{I}$

biceps brachii in, 78,79

biceps cruris, in, 152

deltoid function in, I 7

flexion of elbow and supination of forearm differentiated between in, 79

muscular system of, 3,35

patency of internal abdominal ring in, 243,244

pyramidalis in, $23^{\circ}$

semi-tendinosus and semi-membranosus in, ${ }^{5} \mathrm{I}$

Median nerve, division of, 29

muscles supplied by, movements, 85,86

paralysis, $84-96$

cases of, re-education in, 93

movements of thumb and fingers in, 125

position of anatomical rest in, 9I

Median and musculo-spiral nerve, paralysis, position of anatomical rest in, 93

Median and ulnar nerve, paralysis, position of anatomical rest in, 62

Median and ulnar nerves, complete division, followed by repair of ulnar, with portions of internal cutaneous, median, and great sciatic, 106, 107 
Metacarpo-phalangeal joints, action of extensor communis digitorum at, II6

flexion at, I I6

flexure from over-extension, roo hyper-extension at, II 6

two inner, hyper-extension at, in ulnar paralysis, $\mathrm{IO}_{4}$

Metacarpus, separate extending muscle possessed by, II 3

Metal splint, adjustable, for rest of fingers in re-education of interossei, 108

("dropped wrist"), II 8

Monotremes, biceps cruris in, I52 pyramidalis in, 230

Motion, source of, I9

Multifidus spinæ, action, 208, 2 I 7 divisions of, 208

origin and insertion, 2 I5

Muscle, chronic shortening, 22

contraction, 22, 27

cause, 24,25

treatment, 24

nature of, $\mathbf{I}$

origin, 2,5

Muscles, origin, adaptation to alterations of distance, 43

" survival value," 34

Muscular action, evolution of, I4, I6 testing for, 28

Muscular power, I

Muscularity, definition of, 3

Musculo-cutaneous nerve, origin, $7 \mathrm{I}$ paralysis, positions of rest in, how effected, 80

Musculo-spiral nerve, muscles supplied by, III

origin of, II I

paralysis, I I I

See also Paralysis, musculo-spiral

Musculo-spiral and median nerve, paralysis, position of anatomical rest in, 93

Musculo-spiral and ulnar nerve paralysis, position of anatomical rest in, 92

Myositis, syphilitic, 28

Neck, muscles moving, 206 dorsal, post-vertebral, or extending, 206

fixed point of attachment for, 213

ventral, pre-vertebral, or flexing, 209

muscles of, weakness, rare in infantile paralysis, 2 I9

Nerve cells, "irreparable damage" to, 15
Nerve-supply, double, of index and middle fingers, 107

Nerves, contraction regulated by, I repair after division of, 29

will power conveyed by, I9

See also Median nerve; Musculospiral nerve; Peroneal nerve ; Ulnar nerve; Sciatic nerve, great

Nervous mechanism, constituent parts of, I9

Obturator externus, origin and insertion, I42, I43

internus, insertion in common with superior and inferior gemellus, I $_{42}$

Occiput, articulation of atlas with, $2 \mathrm{OI}$

direct connection of axis with, $20 \mathrm{I}$

and atlas, articulation between, flexion and extension at, example of first order of leverage, 205

Odontoid process, 201

Olecranon, comparison with patella, I 56

Opponens pollicis, action, 87, 9r

Orthograde posture, I 7

Os calcis, function, I 7 I

in African and European races compared, I7 I

Osmosis, 47

Oxidation in relaxation of muscle, 47

Paralysis, grafting in, 18

infantile, cause of " paralysis" of leg and foot muscles, I93 early, rest in bed in, 60

in relation to paralysis of quadriceps, I60, I6I

inutility of upper limb in, cause, 91

re-education of upper extremity in, 65

results of neglected or insufficient rest in, 22O, $22 \mathrm{I}$

weakness of neck muscles rare in, 219

See also Poliomyelitis

ischæmic, 28

musculo-cutaneous, II2

ideal recovery aimed at, 80

positions of rest in, 80

re-education of muscles in, method, 80 
Paralysis, musculo-spiral, I I I

anatomical rest in, 117

best positions for, I 18

appearances, II 3

contracture of flexors in, 118 , I2O, I24

extension of wrist and hand in, 120

movements of thumb and fingers in, 124,125

recovery from, chief factor preventing, I 8

maximum, test for, 120

re-education of muscles in, daily time required for, 122 method, I I 9

of deltoid, 23

point of support, 2 I

test for, 30

of flexors of ankle, 23

of median nerve, $84-96$

cases of re-education in, 93

movements of thumbs and fingers in, 124

position of anatomical restin, 9I

of median and musculo-spiral nerve, position of anatomical rest in, 93

of pronators of forearm, 23

of quadriceps, 23, I60

test for, 30

of serratus, $5^{8}$

of ulnar nerve, 97

anatomical rest in, IOI

hyper-extension of two inner metacarpo-phalangeal joints in, $\mathrm{IO}_{4}$

movements of thumb and fingers in, 124,125

partial, condition of fingers in, IO9

symptoms of, $\mathrm{IO}_{5}$

position of rest in, $\mathrm{IO}_{2}, \mathrm{IO}_{3}$

recovery of interossei in, Io6

re-education of muscles in, methods, IO3

weakness of interossei in, $\mathrm{IO}_{4}$ and median nerve, position of, anatomical rest in, 92

and musculo-spiral nerve, position of anatomical rest in, 92

phrenic, rarity, 248,249

referable to loss of function, ${ }_{5}$

Paraplegia, spastic, of children, contraction of gracilis in, ${ }_{4} 8$

Paresis of muscles elevating limb, cause of stiff shoulder, 68

Patella, fracture of, production by quadriceps, I 59

special function of, I 55, I $_{5} 6$, I $_{57}$
Pectineus, origin and insertion, 139

Pectoral contraction, 67,68

Pectoralis major, chronic shortening of, 23 origin and insertion, 54

Pectoralis minor, action, $5^{6}$ origin and insertion, 57

Pelvic tilting, how produced, 2 I9, 22O, $22 \mathrm{I}$

Peritonitis, acute, movement of thoracic at expense of abdominal muscles in, $24^{\circ}$

Peroneal nerve, injury of, movements lost through, I93

shell wound of upper leg involving, incapacity resulting from, 193

method of rest treatment of foot for, I93, I 94

trauma or gunshot injury of, cause of "paralysis" of leg and foot muscles, I 93

Peroneus brevis, comparative anatomy of, $\mathrm{I} 8 \mathrm{I}$

direction of expansion from tendon, ${ }^{7} 7 \mathrm{O}$

functions, I73, I 77,179, I 80

origin and insertion, 170

Peroneus brevis and longus, in man, compared, I8I

Peroneus longus, almost nonexistent in kangaroo, I8I

functions, $173, \mathrm{I} 77, \mathrm{I} 78, \mathrm{I} 8 \mathrm{O}$

origin and insertion, I73

position, I73

tendon of, course, I 73

Peroneus tertius, functions of, 173 , I 78, I 80

muscle in wombat representing, 170

origin and insertion, $x 69$

Phalanges, middle and distal, lumbricales not extensors of, 90

of toes, middle and distal, motor power for extension of, 189

proximal and distal, extension and flexion, IOO

Phalanx, distal, effect of flexion of first or proximal interphalangeal joint on, "TOO

Plantaris, I 7 I, I 72

function, $I 73, x_{74}$

tendon of, I 72

Plants, power of motion in, 26

Plaster of Paris, uselessness of in rest of joints, 224, 226

Platypus, abdominal muscles in, 230 action of quadriceps in, 16

gluteus maximus and biceps cruris of, 134,135 
Platypus, gracilis of, $I_{4} \mathrm{I}, \mathrm{I}_{5} \mathrm{I}$ male, inguinal region in, 242 muscles on ventral surface of thigh in, I 53

quadriceps in, I6I

thymus gland in, 35

Poliomyelitis affecting abdominal muscles, rest treatment, 239

causing paralysis of quadriceps extensor, 160

deformities of foot following, I 76

"paralysis" of back muscles due to, 220

pelvic tilting resulting from, 220 , 22 I

thymus gland in, 36

See also Paralysis, infantile.

Popliteal nerve, injury of, paralysis resulting from, rest treatment of foot in, I95

Popliteus, action, I49

direct pull of, I 50

origin and insertion, I49

production of inward rotation at knee by, I 5 I

Poupart's ligament, 228, 229

function, 229

Pressure, continuously applied, results of, 26

Processus vaginalis, non-occlusion, resulting in inguinal hernia, 242

Profundus tendon, double supply to middle finger, ro8

Pronation, recovery of, in median paralysis, re-education for, 93

Pronator quadratus, 86,87

action, 77

Pronator teres, 86,87

centre of motion for, 77

contracted lengthening, in cases of old paralysed biceps, 83,84 course, 76

Pronators of forearm, paralysis of, 23

Psoas irritation, testing for, I 33

Psoas major, of man, contraction of, diagnostic significance, I 32 origin and insertion, 130

selection and enlargement in man, I 3 I

Psoas minor, action, 2 I 8

diminution or disappearance in man, I3I

great size in kangaroo and platypus, I3 I

Pulleys, examples of, 8

Pyramidalis, in monotremes and marsupials, 230

origin, 230

Pyriformis, origin and insertion, $\mathrm{I}_{42}$
Quadratus femoris, I 43

Quadratus lumborum, 2 I 7,2 I 8

action, 2 I 8

contraction, 218

origin, attachment, and insertion, 2 I 8

Quadriceps extensor, action of, I4, I 6

fulcrum for action of, $15^{8}$

functions of, $5^{\circ}, I_{57}, I_{5}^{8}$

evolutionary aspect, 160, I6I

in man and other mammals compared, I6I

increases in man, functional not structural, I3 I

insertion, $155,{ }_{5} 6$

muscles composing, I 54

paralysed, anatomical rest of, method of application, I62 application of abduction splint in, 162

misuse of term, 160

recovery of, I65, I66

re-education, commencing, 163 , I 64,165

knee movements in, 165 , I66, I 67

method, 163 graduated, $163-7$

paralysis, 23, 160

conditions wrongly assumed to be, 160

due to poliomyelitis, I6o

function of, loss and recovery, I60, I6I

infantile paralysis in relation to, 160,161

test for, 30

what constitutes, I60, I6I

production of fracture of patella by, I 59

Quadriceps extensores, great relative size of, reason for, I 59

Radius, muscles inserted into, action, 77

Rectus abdominis, $230,23 \mathrm{I}$

abnormality in, $24^{\circ}$

insertion, $23 \mathrm{I}$

situation, $23 \mathrm{I}$

Rectus capitis anticus major, origin and insertion, 204

Rectus capitis anticus minor, course of, 204

Rectus femoris, heads of origin, I 54 insertion, I 54

Rectus lateralis, action, $2 \mathrm{O}_{5}$

origin and insertion, 204, 205

Rectus posticus major, 203

Rectus posticus minor, action, 203 
Re-education, commencing in weakness of extensors of fingers, 107, I08

for muscular weakness in shoulder region, method of, 60-67

for radial or ulnar deviation of haind, II 4

graduated, 29

in cases of median paralysis, 93

of muscle function, 20,22

of muscles acting on foot and toes in sciatic nerve paralysis, I 92, I94, I95

of muscles in musculo-cutaneous paralysis, method, 80

in ulnar paralysis, $\mathrm{IO}_{3}$

of finger after suture of ulnar nerve after complete division, I IO

of paralysed quadriceps extensor, I63, I64-I67

Reflex movements, I6I

Relaxation, physiological action of, 36

stimulus to, 40

Renuentes, 203

Reptiles, abdominal situation of lungs in, 235

Respiration, abdominal, diminution of, grave import of, $24^{\circ}$ muscles of, $228-49$

principal muscle of, 234

Respiratory mechanism, voluntary and involuntary action of muscles in, 234

Respiratory movements, essence of, 234 of abdominal viscera, 236

Rest, anatomical, in musculo-spiral paralysis, I I 7

and re-education, of muscles acting on foot and toes in sciatic nerve paralysis, 192 , I 94, I 95

recovery from " paralysis" of right foot due to infantile paralysis, after, I 95, I 96

result in case of shell wound injury of great sciatic nerve, 196

in treatment, $19,2 \mathrm{I}$

of joints, positions for, 222-7

of muscles, what constitutes, 20

zero position, I 8

physiological, i 8

treatment of poliomyelitis affect-

ing abdominal muscles, 239

zero position of, I 8,2 I

Reverse action, muscular, $4 \mathrm{I}$
Rhomboids, action, $5^{6}$

antagonist, $5^{6}$

origin and insertion, 57

Ribs, action, centre of motion for, 2 I 3,247

function, 246,247

true muscles of, 232

See also Cervical ribs

Riding, muscles used in, I39

Riolan, function of serratus posticus inferior, 248

Rotatores, action, 2 I 7 spinæ, 2 I 5

Running, muscles employed in, I 74, I 80

Russell, Hamilton, cause of inguinal hernia in children, $24 \mathrm{I}$

Sacro-iliac joint, disease of, psoas contraction diagnostic of, I 32

Sarcolemma, I

Sartorius, fulcrum or centre for action of, 147

function in kangaroo, ${ }^{5} 5$

origin, insertion, and functions I $_{4} 6, I_{47}$, I $_{4} 8$, I $_{5} \mathrm{I}$

reverse action of, 42

Scaleni, 209

action, 2 I I, 2 I 2

course, 2 I I

Scalenus anticus, 2 I I

medius, 2 I I

posticus, 2 I I

Scapula, elevation of, in paralysis of serratus, $5^{8}$

muscles acting on, 56

paralysed, re-education, 64

rotation, $57,5^{8}$

arc of movement in, 61,62 , 64,65

winging of, $5^{8}, 60$

Sciatic nerve, great, in thigh, injury of, mischief resulting from, I 93

paralysis following, treatment, I95

rest and re-education of muscles acting on foot and toes in, I92, I 94, I 95

severance by shell wound, result of treatment by rest and reeducation of muscles, 196

trauma or gunshot injury of, cause of paralysis of leg and foot muscles, I93

Semi-membranosus, origin and insertion, $\mathrm{I}_{4} 8$

Semi-spinales, 207

action, 217

on one or both sides, 208 
Semi-spinales, not found in lumbar region, 2 I 7

origin and insertion, 208

Semi-spinalis, course, 2 I 5

Semi-tendinosus, origin and insertion, $\mathrm{I}_{4} 8$

and semi-membranosus in marsupials, I $5 \mathrm{I}$

Serratus, loss of muscular power in, position of anatomical rest for, 59,60

paralysis of, $5^{8}$

Serratus magnus, or anterior, action, 56

origin and insertion, 56, 57

Serratus posticus inferior, 233 function, 248

Serratus posticus superior, 233

function, 247

Sesamoid bones, 8

development, I 84, I 85

Shell wound causing severance of great sciatic nerve, result of treatment by rest and reeducation of muscles, I 96

of upper leg involving peroneal nerve, incapacity resulting from, I 93

Shoulder-joint, diseases and injuries, involvement of long head of biceps in, 79

excision of, division of long head of biceps avoided during, 75

loss of muscular power in, benefit of rest in bed during, 60

Shoulder region, in man, muscles of, $5^{2}$

loss of muscular power in, positions of anatomical rest for, $5^{8}$

muscular weakness in, re-education for, method, 60-7

Sitting, action of knee flexors in, I 50

flexion and extension of leg on thigh during, I $5^{\circ}$

flexion at hip-joint during, I 50

Skeletal balance apart from muscular action, non-existent, 2 I 9

muscles, number and weight of, I

Soleus, function of, I 73, I 74

knee joint not crossed by, I 7 I origin and insertion, I $7 \mathrm{I}$

Spastic diseases of children, impediment to walking in, $\mathrm{I}_{4} \mathrm{O}$

Spinal cord, relation to muscular action, I6I

Spinalis dorsi, origin and insertion, 2 I 5

Spine, area of greatest mobility of, 206

centre of motion of ribs at, 2 I 3
Spine, cervical, lateral movement, how effected, 2 I 2

effects of flexion and extension of hip, on, I 32

elasticity, 2 I 3,2 I 4

fixed point for action of intercostal muscles, 2 I 3

flexion movement in, area where greatest, 2 I 3

front of, muscles on, for flexion few, for extension many, 2 I 8

lateral curvature of, due to insufficient rest in infantile paralysis, 220

movements of head on, 201

muscles of, 20O-5

post-vertebral or extending, 2OI, $2 \mathrm{O} 2$

pre-vertebral, or bending, $2 \mathrm{O}_{4}$ weakness (" paralysis ") due to poliomyelitis, 220

treatment by rest of trunk and lower limb in double Thomas's splint, 220

thoracic, fixed point for attachment of muscles acting on neck, 2 I 3

thoracic region, flexion and extension practically absent in, 2I 3,217

no pre-vertebral muscle in, 2 I 7

Splenius capitis, $2 \mathrm{O} 2$

action, $2 \mathrm{O} 2$

origin and insertion, $2 \mathrm{O} 2$

Splenius cervicis, 206, 207

action of one or both muscles, 207

origin and insertion, 207

Splenius colli, $2 \mathrm{O} 2$

Splint, " cock-up," for rest of hand in paralysis of wrist extenders, I I 8 , I I 9

See also Aluminium " cockup " splint

dorsal hip (Thomas's), I 33

for abduction of upper limb, in ulnar paralysis, IO3

Lister's, for rest of wrist-joint, 226

method of application in rest of foot, in gunshot injury of peroneal nerve, I93

Thomas's, double, for rest of trunk and lower limbs in "paralysis" of back muscles 220

single, with arc shoe attached, for rest of knee joint, 225

Standing erect, muscles used in, I 39 on tiptoe, muscles employed in,

I 74, I 80 
Sterno-clavicular articulation, connection of upper extremity with trunk at, 57

Sterno-mastoid, fulcrum or centre of motion of, 205

origin and insertion, 205

Sterno-mastoids, action of one or both contrasted, 205

Stiff shoulder, 67,68

causal factors, 68

treatment, 69,70

Structural correlations, 30

Sublimis, contraction in ulnar paralysis, how resulting, Iro

Subscapularis, origin and insertion, 55

Superior oblique, action, $2 \mathrm{O}_{4}$ origin and insertion, 203

Supinator brevis, centre of motion for, 77

course, 76

insertion, $\mathrm{Ir}_{3}$

order and position of origin, I I I power, II2

re-education in musculo-spiral paralysis, I22

Supinator longus, 34

Supinators, stimulus to contrac. tion of, $4 \mathrm{I}$

Supracostales. See Levatores costarum

Supraspinatus, action, $5^{\mathrm{I}}$

function, 57

origin and insertion, 54

"Survival value" of muscles, 34

Synergists, examples of, 45

Syphilitic myositis, 28

Talipes calcaneus, deformity of foot present in, $r_{7} 6, r_{79}$

rest treatment, 192

result of poliomyelitis in childhood, 176

Talipes equinus, deformity of foot present in, $I_{7} 6, \mathrm{I}_{79}$

result of poliomyelitis in childhood, 176

treatment, 24

by rest, 192

Talipes valgus, I79 treatment, $\mathrm{I} 8 \mathrm{I}$

Talipes varus, 179 in children, treatment, 183

Tasmanian devil, cremaster muscle in, 244

muscular system of, 3

Tendinous insertion, 2

Tendo achillis, chronic shortening of, 23

contracted chronically, 24
Tendo achillis, contraction, prevention, 27

formation, I 7 I

muscles acting through, functions, 174

superficial portion, I 7 I

and tibialis posticus, contraction of, treatment, 194

Tendon, force and leverage due to, 3

insertion into bone, 3

of extensor communis digiti, how elongated, II 7

position and shape, II 6

of origin of muscle, 2

of peroneus longus, course, $\mathrm{I}_{73}$

fibro-cartilage formed in, $x_{73}$

of plantaris, $\mathrm{I} 2$

of tibialis posticus, $\mathrm{r} 72, \mathrm{r} 73$

shortening of, 24

tissue, limitations of, 3

transplantation, in thumb drop

with paralysis of muscles, 113

Tendons, elongation of, 27

long, of interossei, IOI

of extensor communis digitorum, I I 4

of foot, treatment of, inequinus, 24 of gastrocnemius, $\mathrm{r} 7 \mathrm{O}$

of interossei, connections with communis tendons, II 6

how elongated, II 7

Tensor fasciæ femoris, origin, insertion, and work of, $\mathrm{I}_{4} \mathrm{r}, \mathrm{r}_{4} 2$

Teres major, function, 55

origin and insertion, 55

tendon, 55

Teres minor, origin and insertion, 54

Test for action of flexors, 29

for deltoid paralysis, 30

for muscle action, 28

for paralysis of brachialis, 9

for paralysis of quadriceps, 30

volitional, 16,28

Testes, descent of, $24 \mathrm{I}$

Thigh, abduction of, I4I

action of adductors on, 139

external and internal rotation, muscles effecting, $\mathrm{r}_{42}$

flexion, pathological conditions associated with, $\mathrm{r}_{32}$

flexion and extension of leg on, during sitting, ${ }_{1} 5^{\circ}$

of trunk of body on, 129

muscles of, $127-142$ regions of insertion, 128

on ventral surface of, in platypus, ${ }_{53}$

and trunk, rigid, bending back at knee joints by acrobat and athletes, ${ }_{5} 6$ 
Thomas, H. O., angle of flexion for rest of elbow joint, 227

method of resting hip joint, I32, I33

treatment of "dropped wrist," I I 9

Thomas's splints. See Caliper splint, Dropped wrist metal splint, Hand splint, Splint, Thomas's

Thorax, muscles of, 232

function, 246

movement at expense of abdomina!, in inflammatory conditions of abdomen, 240

Thumb, abduction of, important factor in, 126

drop with paralysis of muscles, tendon transplantation in, I 3

extension, in musculo-spiral paralysis, II 7

flexion, in ulnar paralysis, IO2, IO4

long abductor of, rest in musculospiral paralysis, I I 7

motor forces moving, 86

movement in human hand, specialization, how manifested, I 3 .

movements in median, ulnar, and musculo-spiral paralysis, I24-6

muscles of, loss of function in, 223

re-education in ulnar paralysis, $\mathrm{IO}_{4}$

opposition, 87

and flexion, recovery of in median nerve paralysis, reeducation for, 95

small muscles of, 98

Thymus gland and development of muscle, 34

use of, in poliomyelitis, 36

Tibia compared with fibula, I7 relation of olecranon to, I 56

Tibialis anticus, division in talipes varus, 183

functions, $173,177,178, \mathbf{1} 80$

in saltatorial kangaroo, $I_{7} 8$ origin and insertion, 168

Tibialis posticus, absence in kangaroo, 178

functions, I73, I 77, I 78, I94

origin, 172

tendon of, 172

insertion, $\mathrm{I}^{\prime} 73$

and tendo achillis, contraction of, treatment, 194
Toe, big, bunion of, $\mathrm{I} 86$

flexors of, action improved by leverage, 185

two separate extending muscles of, 183

See also Hallux

little, adducting power of, I86

production of extension in, 189

Toes, abductors and adductors of, I9I

even extension, I 89

flexors of, action, I 90

four lesser, abductors and adductors of, I9I

extensors of, 188

flexors of, 189

muscles acting on, I 88

lateral action, imaginary line for, I9I

muscles of, 182

Trachelo-mastoid, action, 203

origin and insertion, $2 \mathrm{O}_{3}$

Tracheotomy in epigastric retraction, 249

Transversalis, aponeurosis of, 229

abdominis, action, 248

cervicis, 206, 207

action, 207

origin and insertion, 207

origin, 229

sheet, primitive, muscular system developed from, $23^{8}$

Transversus pedis, 186

Trapezius, action, $56,5^{8}$

function, 56

loss of muscular power in, treatment by abduction of arm, $5^{8}$ origin and insertion, 56

Trapezoid, 57

Triangular fascia, 230

Triceps, III, II 2

anatomical rest of, in musculospiral paralysis, I I 7

antagonist of, II2

origin of, III, II 7

power of, I I 2

re-education in musculo-spiral .paralysis, 123

relaxation does not permit of supination, 78

stimulus to contraction of, $4 \mathrm{I}$

Trunk of body,flexion and extension on thigh, 129

and thigh, rigid, bending back at knee joints by acrobats and athletes, 150

Ulnar nerve, branches arising in forearm, 97

division of, 29 
Ulnar nerve, origin, 97

paralysis, 97

anatomical rest in, IOI

movements of thumb and

fingers in, I24, I2 5

position of rest in, IO2, 103

re-education of muscles in, methods, IO3

suture for complete division, followed by early re-education of muscles, I IO

Ulnar and median nerve paralysis, position of anatomical rest in, 92

Ulnar and median nerves, complete division, followed by repair of ulnar, with portions of internal cutaneous, median, and great sciatic, 106

Ulnar and musculo-spiral nerve, position of anatomical rest in, 92

Upper limb abduction splint, 227

in ulnar paralysis, IO3

Vastus, externus or lateralis, crigin and insertion, $\mathrm{I}_{54}$

internus or melialis, connection with crureus, ${ }_{54},{ }_{55}$ extension of, ${ }_{55}$ origin, ${ }_{54}$

Vertebre, individual motion between, limited, 213

lumbar, disease of, psoas contraction as diagnostic of, $\mathrm{I}_{2} 2$

Viscera, support of, by abdominal muscles, 239

Visceroptosis, cause of, 240

cause of greater frequency in women, $240,24 \mathrm{I}$

Volition, normal muscle contracted by, 25

Volitional test, I6

for muscle action, 28
Walking, impediment to in spastic diseases of children, 140 muscles employed in, 174,180

Will power, conveyed by nerves, I 9

Wombat, biceps brachii in, 78,79

brachio-radialis in, 73

cremaster muscle in, 244

dissection of inner side of knee in, 152

male, inguinal region in, 245 patent internal abdominal ring and canal in, 243

muscle representing peroneus tertius in, I 70

muscular system of, 3,35

peroneus longus in, $18 \mathrm{I}$

Women, cause of greater frequency of visceroptosis in, $24 \mathrm{O}, 24 \mathrm{I}$

frequency of hallux valgus in, 187

Wrist-joint, diseased or injured, splints for use in rest of, 226 extension, position of, effect on flexion of fingers in ulnar paralysis, 109

promoting improvement of flexor leverage of fingers, 96 extensors, abduction power, II 3 , II 4

abduction power, in connection with re-education for radial or ulnar deviation of hand, I I 4 order and position of origin, I I I, I I 2

paralysis of, splint for rest of hand in, I 18

flexion, in ulnar paralysis, ror recovery of in median paralysis, re-education for, 94

flexors of, testing of, 29

forces acting on, $4^{\circ}$

and hand, extension, in musculospiral paralysis, 120

Zero position of rest, 18,2 I 
H. K. LEWIS AND CO. LTD.

28 GOWER PLACE, LONDON, W.C.I

AND PRINTED BY HAZELL, WATSON AND VINEY, LD , LONDON AND AYLESBURY. 



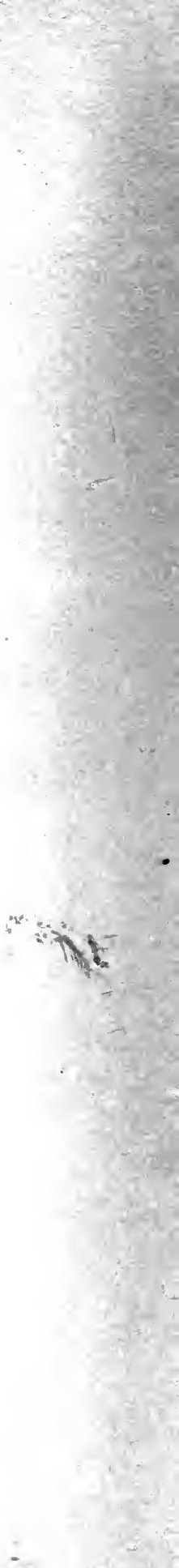



RETURN TO the circulation desk of any University of California Library

or to the

NORTHERN REGIONAL LIBRARY FACILITY

Bldg. 400, Richmond Field Station

University of California

Richmond, CA 94804-4698

ALL BOOKS MAY BE RECALLED AFTER 7 DAYS

- 2-month loans may be renewed by calling (510) 642-6753

- 1-year loans may be recharged by bringing books to NRLF

- Renewals and recharges may be made 4 days prior to due date

\section{DUE AS STAMPED BELOW}

\section{IJAN 172006}




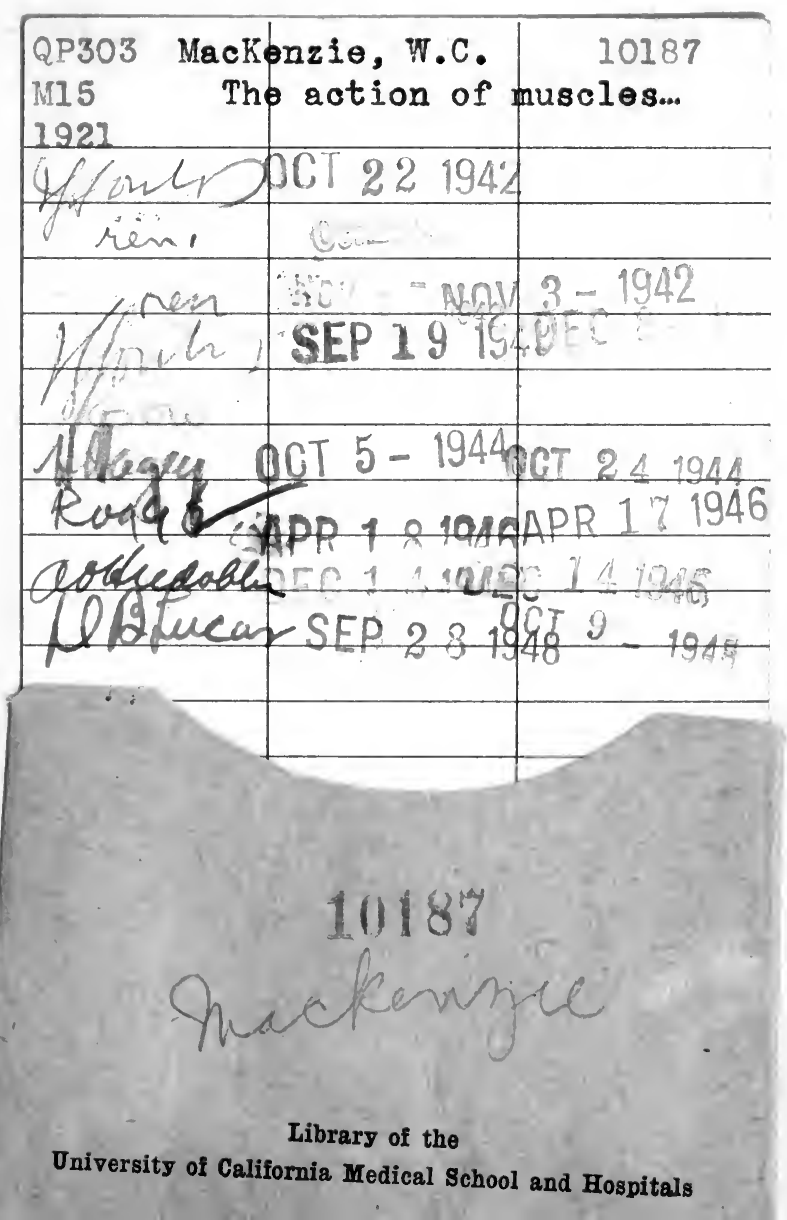


